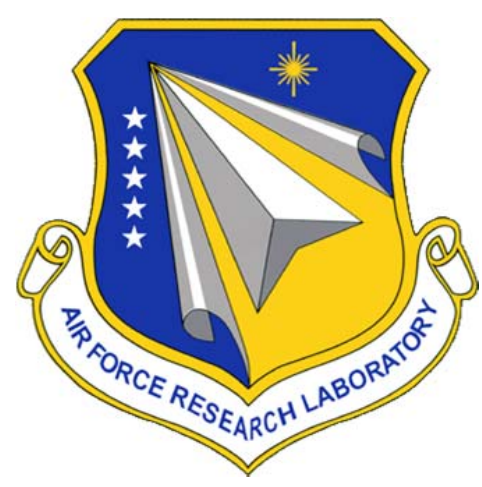

AFRL-AFOSR-UK-TR-2014-0048

Dynamics of flexible MLI-type debris for accurate orbit prediction

Matteo Ceriotti

Gianmarco Radice

THE UNIVERSITY OF GLASGOW

UNIVERSITY AVE

GLASGOW G128QQ UNITED KINGDOM

EOARD Grant 13-3028

Report Date: September 2014

Final Report from 1 March 2013 to 31 August 2014

Distribution Statement A: Approved for public release distribution is unlimited.

Air Force Research Laboratory

Air Force Office of Scientific Research

European Office of Aerospace Research and Development

Unit 4515, APO AE 09421-4515 


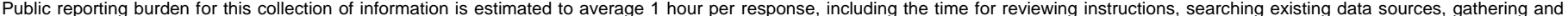

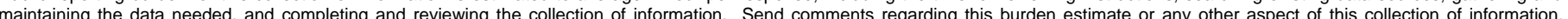

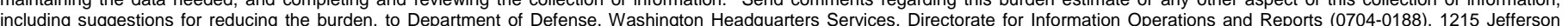

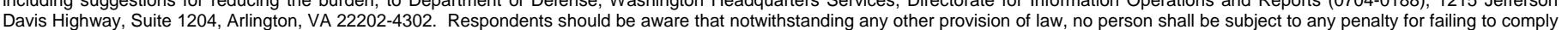
with a collection of information if it does not display a currently valid OMB control number.

with a collection of information if it does not display a currently valid OMB
1. REPORT DATE (DD-MM-YYYY) 15 September 2014

4. TITLE AND SUBTITLE

Dynamics of flexible MLI-type debris for accurate orbit prediction
3. DATES COVERED (From - To)

1 March 2013 - 31 August 2014

\section{5a. CONTRACT NUMBER}

FA8655-13-1-3028

5b. GRANT NUMBER

Grant 13-3028

5c. PROGRAM ELEMENT NUMBER

\section{F}

6. AUTHOR(S)

Matteo Ceriotti

Gianmarco Radice

\section{5d. PROJECT NUMBER}

5d. TASK NUMBER

5e. WORK UNIT NUMBER

\section{PERFORMING ORGANIZATION NAME(S) AND ADDRESS(ES)}

THE UNIVERSITY OF GLASGOW

UNIVERSITY AVE

GLASGOW G128QQ UNITED KINGDOM

9. SPONSORING/MONITORING AGENCY NAME(S) AND ADDRESS(ES)

EOARD

Unit 4515

APO AE 09421-4515

\section{PERFORMING ORGANIZATION REPORT NUMBER}

N/A

10. SPONSOR/MONITOR'S ACRONYM(S)

AFRL/AFOSR/IOE (EOARD)

11. SPONSOR/MONITOR'S REPORT NUMBER(S)

AFRL-AFOSR-UK-TR-2014-0048

\section{DISTRIBUTION/AVAILABILITY STATEMENT}

Distribution A: Approved for public release; distribution is unlimited.

\section{SUPPLEMENTARY NOTES}

\section{ABSTRACT}

This study aimed at developing algorithms for accurate, long term propagation of a particular type of space debris, made of multi-layer insulation (MLI) foils, originated from delamination from ageing spacecraft. This type of debris has high area-to-mass ratio, combined with high reflectivity index, and hence it is subject to strong solar radiation pressure (SRP) acceleration. In addition, MLI membranes are highly flexible, therefore their effective, exposed area to the sun is subject to change over time. This effect has been taken into account in the past through averaging techniques. This study attempts, for the first time, to include the dynamics of the deformation in the propagation of the equations of motion (orbit and attitude). The computational cost of modelling a deformable membrane subject to forces can be significant, hence two different models were developed: one uses a linear Bernoulli-Euler beam model, while the second discretizes the membrane properties through lump masses, spring and damper. The latter framework models arbitrarily large deformations, as expected on the lowbending-stiffness membrane. Propagations of typical geostationary orbit (GEO) debris using both models are compared to other models not taking into account the deformation (cannonball model or rigid membrane). The presented results show that considerable difference in the estimation of the orbital parameters (particularly inclination and eccentricity) can be obtained even over tens of days.

In addition, in order to reduce the computational burden of the equations of motion, a numerical integrator has been developed, being able to treat coupled dynamics with different typical time-scales, specifically orbit and attitude equations. Assuming the attitude is the fast dynamics, faster integration times can be obtained, with the same level of accuracy of a traditional algorithm, if the computational cost of the fast dynamics is above a certain threshold.

15. SUBJECT TERMS

EOARD, orbital debris, HAMR objects, multi-layered insulation, orbital dynamics, orbit predictions, orbital propagation

\begin{tabular}{|l|l|l|}
\hline \multicolumn{3}{|l|}{ 16. SECURITY CLASSIFICATION OF: } \\
\hline $\begin{array}{l}\text { a. REPORT } \\
\text { UNCLAS }\end{array}$ & $\begin{array}{l}\text { b. ABSTRACT } \\
\text { UNCLAS }\end{array}$ & $\begin{array}{l}\text { c. THIS PAGE } \\
\text { UNCLAS }\end{array}$ \\
\end{tabular}

17. LIMITATION OF ABSTRACT

SAR

\section{8, NUMBER} OF PAGES

117 19a. NAME OF RESPONSIBLE PERSON Kevin Bollino

19b. TELEPHONE NUMBER (Include area code) +44 (0)1895616163 


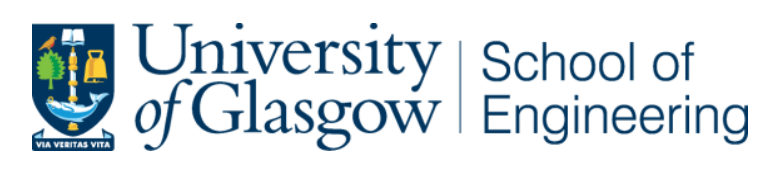

\title{
Dynamics of flexible MLI-type debris for accurate orbit prediction
}

\author{
Final Report
}
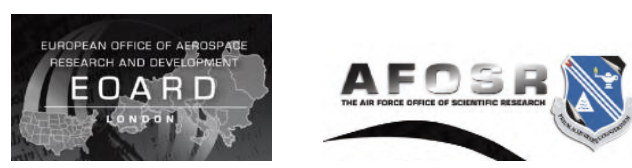

1 March 2013 - 31 August 2014

PI: $\quad$ Dr Matteo Ceriotti, University of Glasgow

CO-I: Dr Gianmarco Radice, University of Glasgow 


\begin{abstract}
This report summarizes methodologies and findings of a study performed at the School of Engineering, University of Glasgow, funded by the European Office of Aerospace Research and Development, during the period March 2013 to August 2014.

The study aimed at developing algorithms for accurate, long term propagation of a particular type of space debris, made of multi-layer insulation (MLI) foils, originated from delamination from ageing spacecraft. This type of debris has high area-to-mass ratio, combined with high reflectivity index, and hence it is subject to strong solar radiation pressure (SRP) acceleration. In addition, MLI membranes are highly flexible, therefore their effective, exposed area to the sun is subject to change over time. This effect has been taken into account in the past through averaging techniques. This study attempts, for the first time, to include the dynamics of the deformation in the propagation of the equations of motion (orbit and attitude). The computational cost of modelling a deformable membrane subject to forces can be significant, hence two different models were developed: one uses a linear Bernoulli-Euler beam model, while the second uses discretizes the membrane properties through lump masses, spring and damper. The latter framework models arbitrarily large deformations, as expected on the low-bending-stiffness membrane.

Propagations of a typical geostationary orbit (GEO) debris using both models are compared to other models not taking into account the deformation (cannonball model or rigid membrane). The presented results show that considerable difference in the estimation of the orbital parameters (particularly inclination and eccentricity) can be obtained even over tens of days.

In addition, in order to reduce the computational burden of the equations of motion, a numerical integrator has been developed, being able to treat coupled dynamics with different typical time-scales, specifically orbit and attitude equations. Assuming the attitude is the fast dynamics, it resulted that faster integration times can be obtained, with the same level of accuracy of a traditional algorithm, if the computational cost of the fast dynamics is above a certain threshold.
\end{abstract}

An appendix shows the validation of the deformation computed through the linear model with the commercial FEM solver ABAQUS. 


\section{Table of Contents}

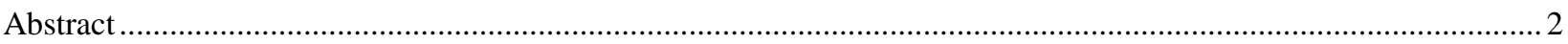

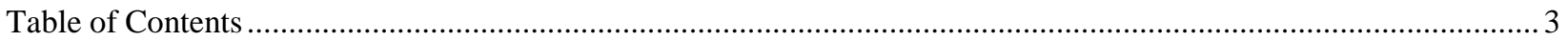

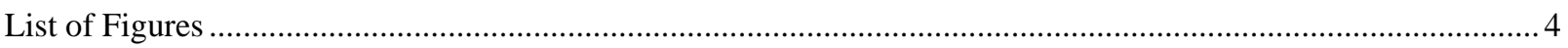

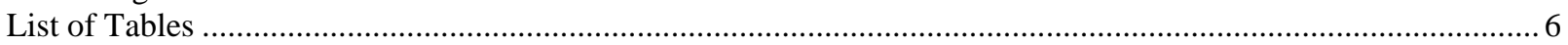

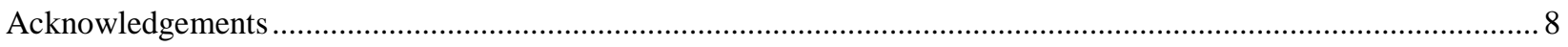

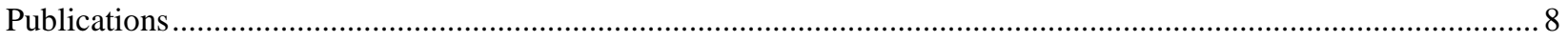

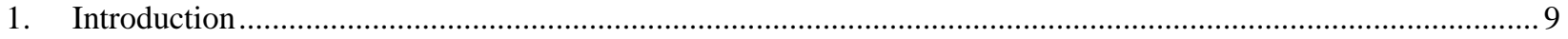

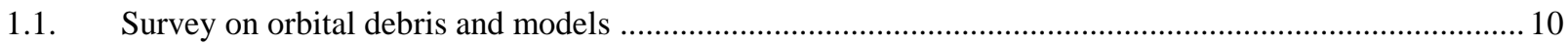

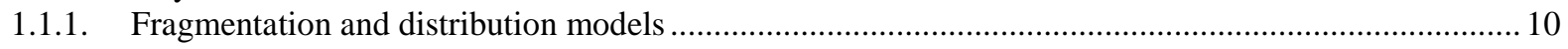

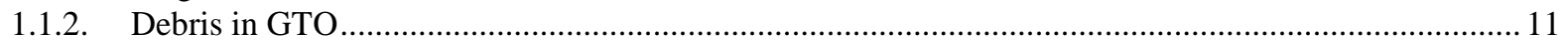

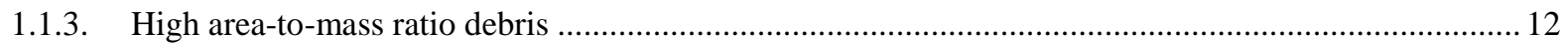

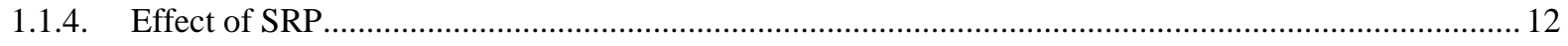

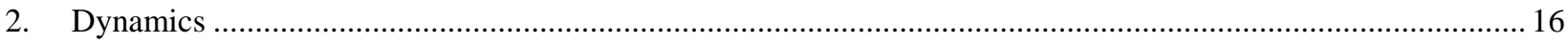

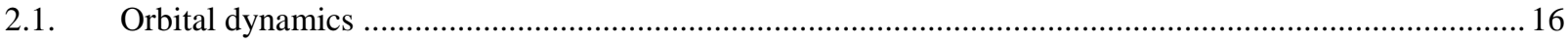

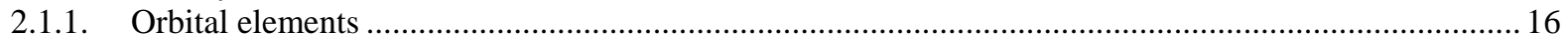

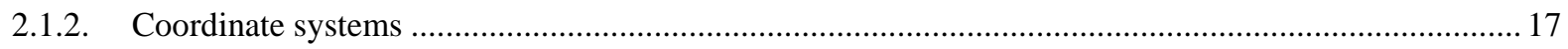

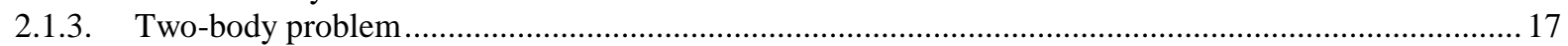

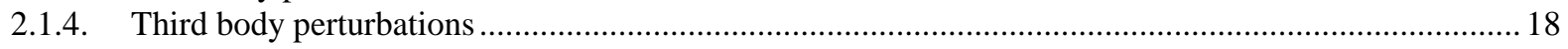

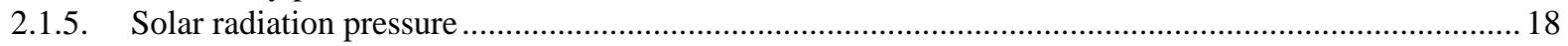

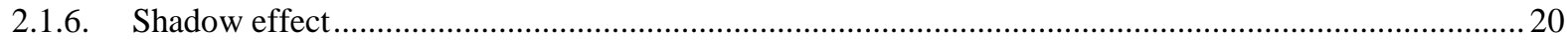

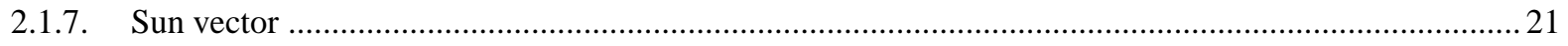

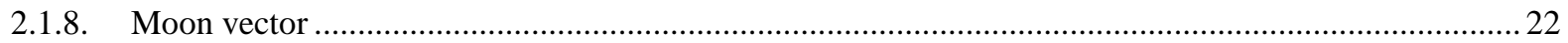

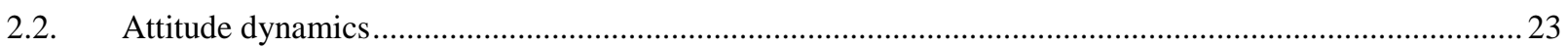

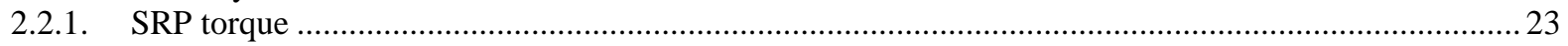

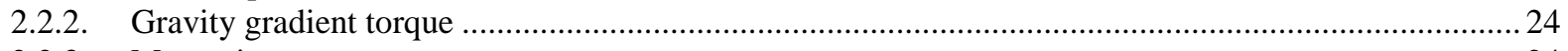

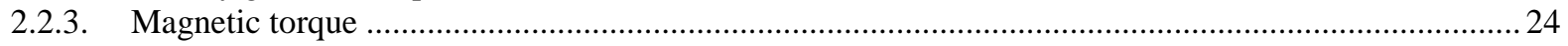

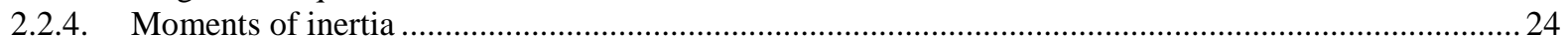

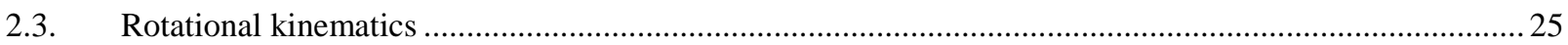

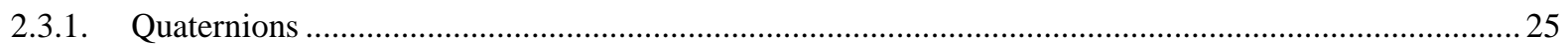

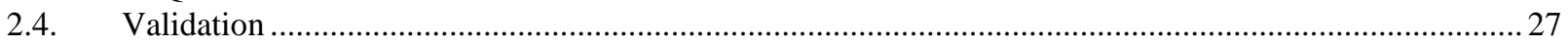

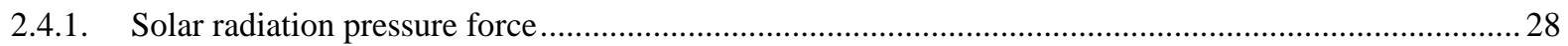

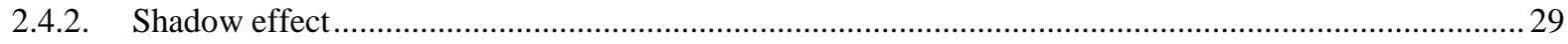

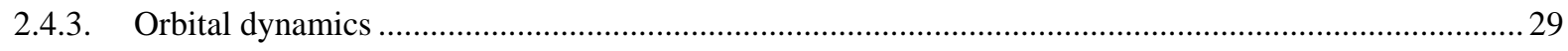

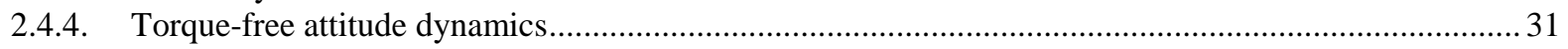

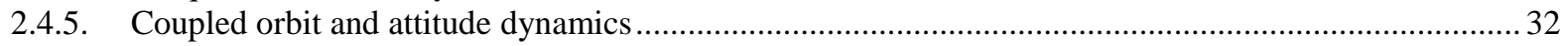

3. Orbital and attitude dynamics of flexible debris - Bernoulli-Euler model ................................................4 41

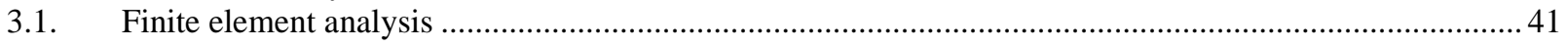

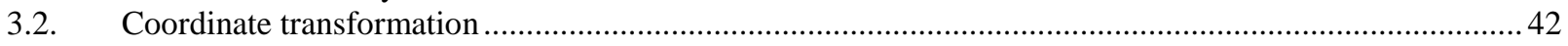

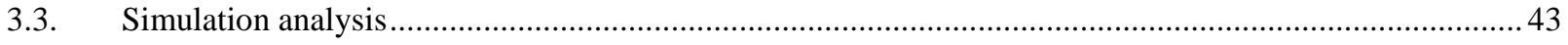

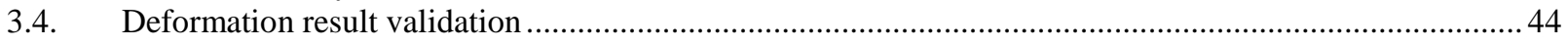

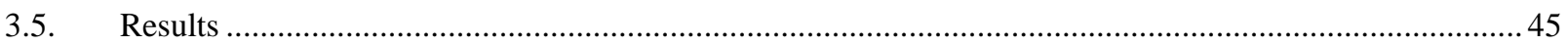

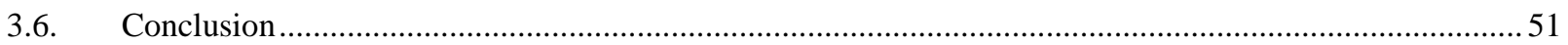

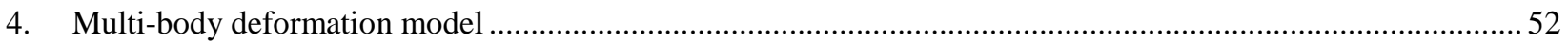

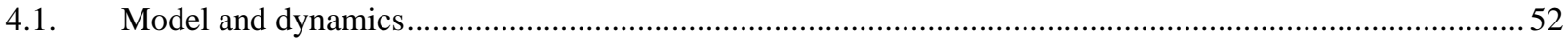

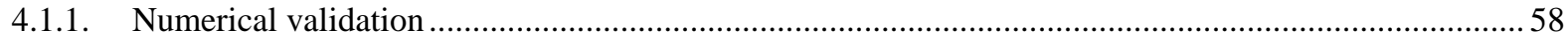

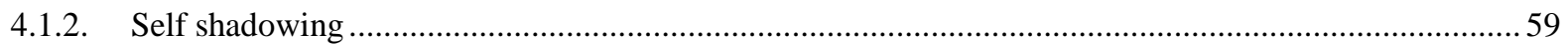

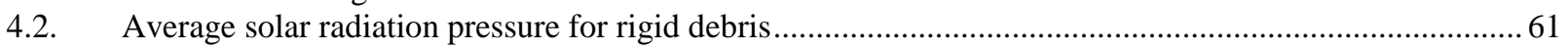

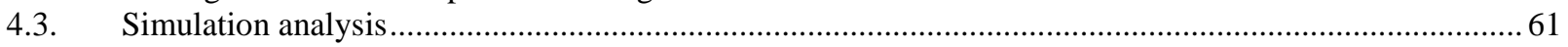

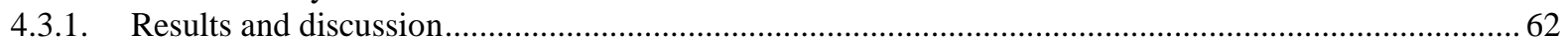

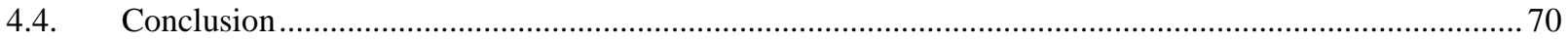




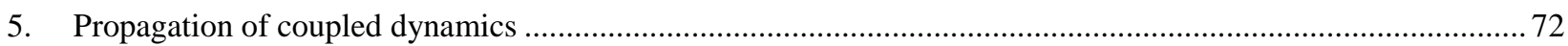

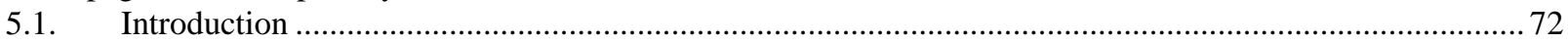

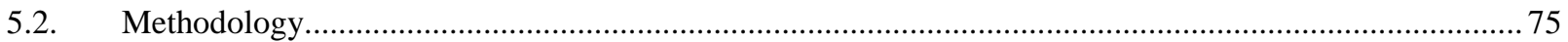

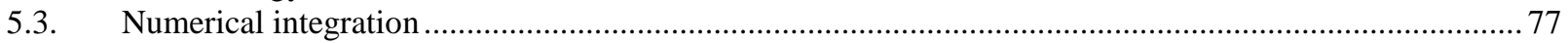

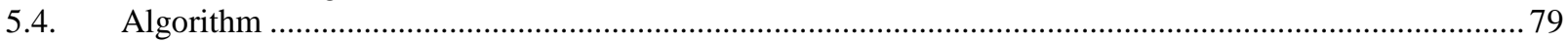

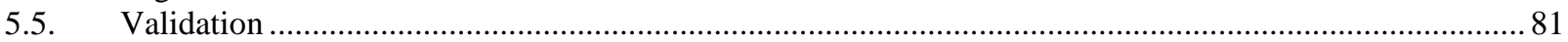

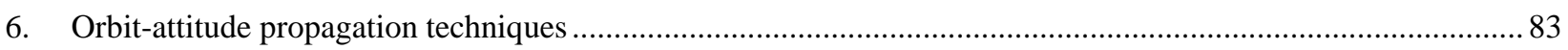

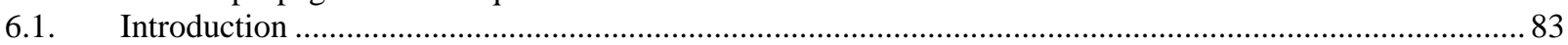

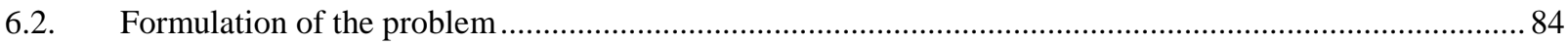

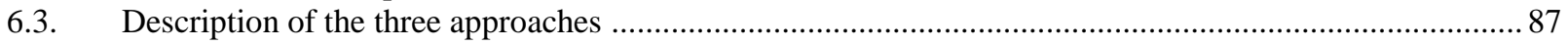

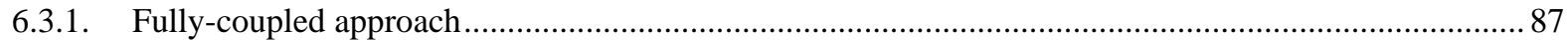

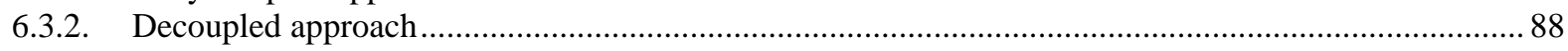

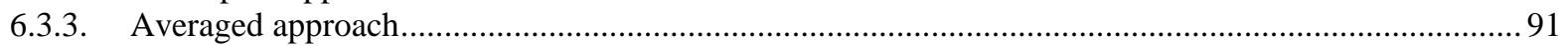

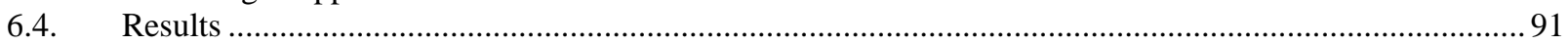

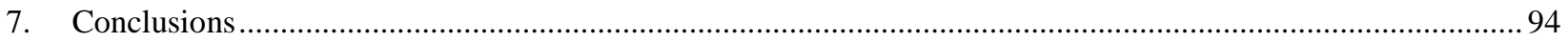

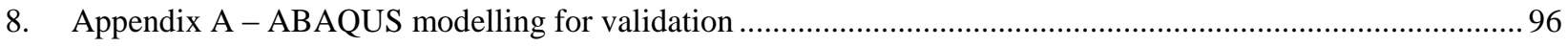

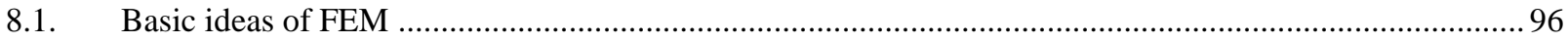

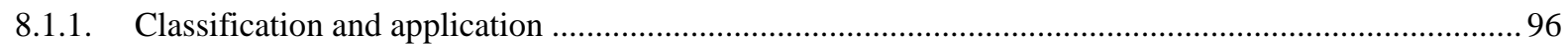

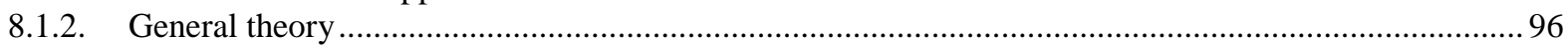

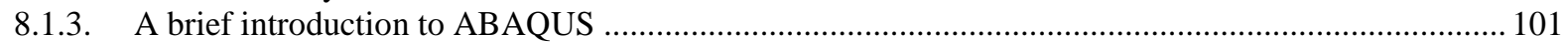

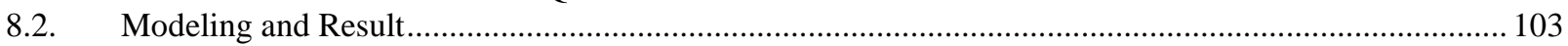

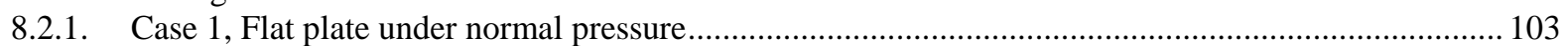

8.2.2. Case 2, Right-angle membrane under constant solar pressure ....................................................... 105

8.2.3. Case 3, Right-angle membrane under changeable load ....................................................................... 107

8.2.4. Case 4, Curve membrane under constant solar pressure ................................................................... 109

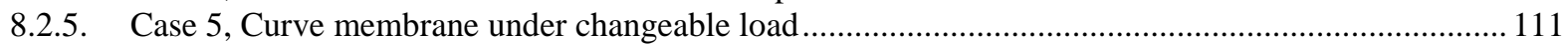

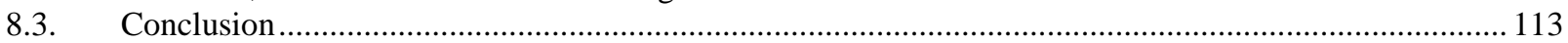

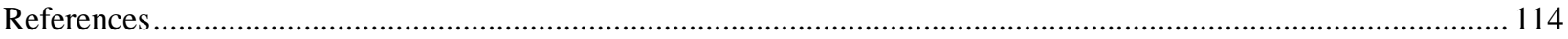

\section{List of Figures}

Fig. 1. Objects in Earth orbit that are currently being tracked. Approximately $95 \%$ of the objects in this illustration are orbital debris [Souce: NASA]. .

Fig. 2. (a) Hole made by debris in the panel of NASA's Solar Max experiment. (b) Window of the space shuttle damaged by a tiny piece of space junk (a paint fleck) during the STS-7 mission (Photo: NASA Orbital Debris Program Office)

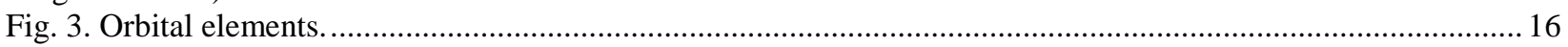

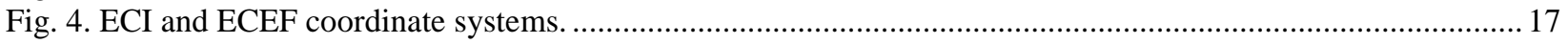

Fig. 5. Gravitational force from the Earth acting on a satellite ...................................................................... 18

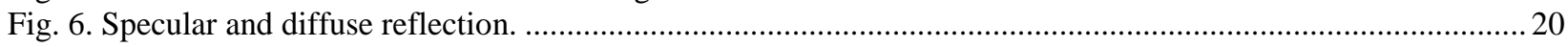

Fig. 7. Incident solar radiation geometry. Note that the unit vectors $t$ and $n$ are the unit vectors in the tangential and normal directions (with respect to the surface), respectively; $h$ and in are oriented upwards from the plane of the paper; $\mathrm{s}$ and $\mathrm{r}_{\mathrm{sat}} \odot$ are collinear with the incident solar rays, pointing towards the sun..............................................2 20

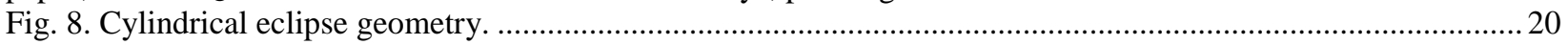

Fig. 9. Eclipse geometry for conical model. ……........................................................................................ 21

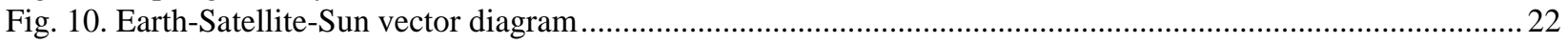

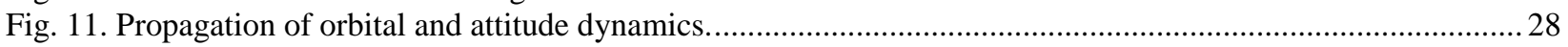

Fig. 12. Solar radiation pressure force result by varying incident angle ............................................................. 28

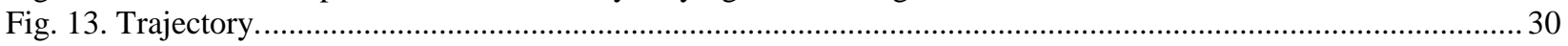

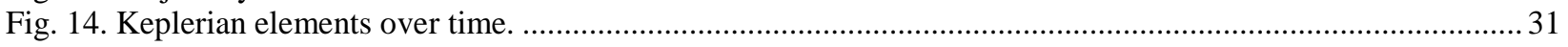

Fig. 15. Trajectory of angular momentum in body frame. ................................................................................. 32

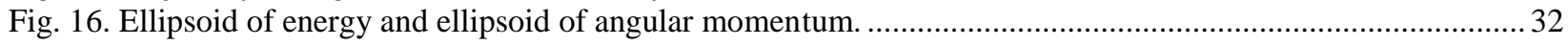

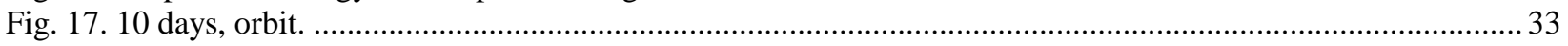

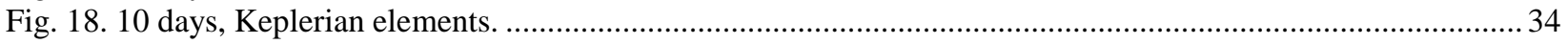




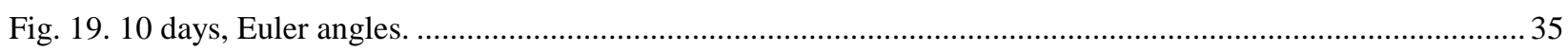

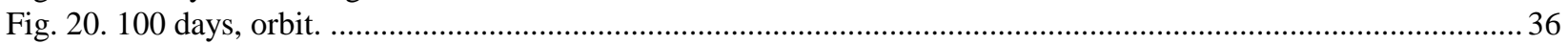

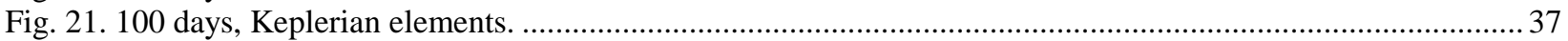

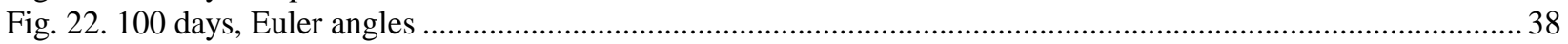

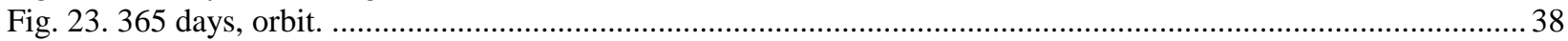

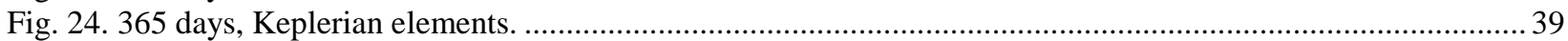

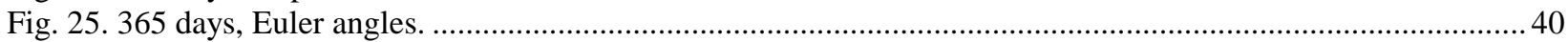

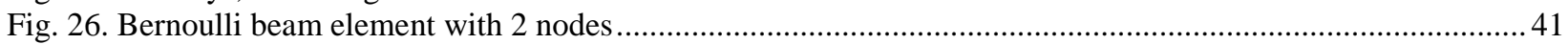

Fig. 27 a) Deformation of folded flat plate at $0,1,2$ and $3 \mathrm{~s}$ by force $8.011 \mathrm{e}-7 \mathrm{~N}$ in the first node in right direction $\mathrm{b}$ )

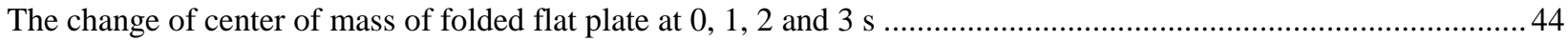

Fig. 28 a) Deformation of flat plate in $0.2,0.4$ and $0.6 \mathrm{~s}$ due to a force of $8.011 \mathrm{e}-7 \mathrm{~N}$ in the first node in -y direction

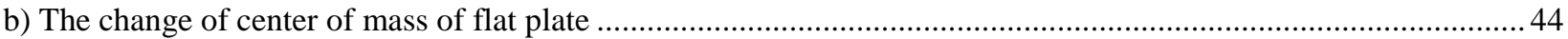

Fig. 29. a) Eccentricity of flat plate and folded up flat plate in both rigid and flexible cases b) Magnify eccentricity

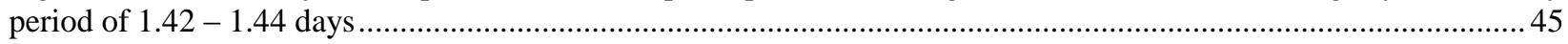

Fig. 30. a) Inclination of flat plate and folded up flat plate in both rigid and flexible cases b) Magnify inclination

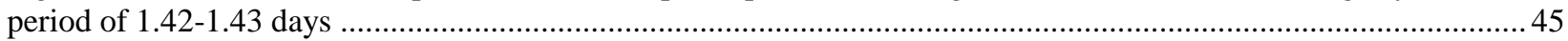
Fig. 31. a) Eccentricity of flat plate in both rigid and flexible cases in 150 days b) Eccentricity of folded flat plate in

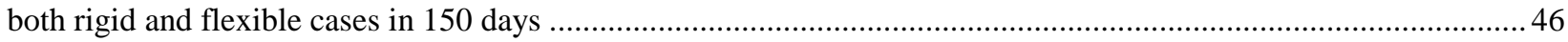
Fig. 32. a) Inclination of flat plate in both rigid and flexible cases in 150 days b) Inclination of folded flat plate in

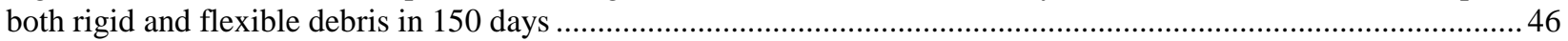

Fig. 33. Euler angles of flat plate in case of rigid body and flexible body in 3 days ..............................................4 47

Fig. 34. Euler angles of folded flat sheet in case of rigid body and flexible body in 3 days ...................................... 48

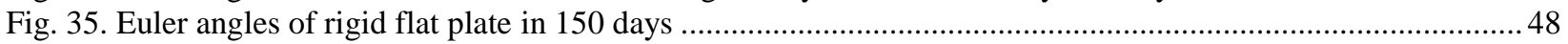

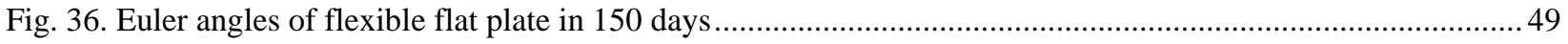

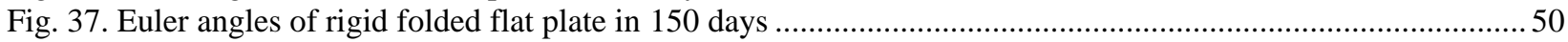

Fig. 38. Euler angles of flexible folded flat plate in 150 days........................................................................... 51

Fig. 39. a) The membrane for 2D deformation is modeled with series of masses including torsional springs and dampers. b) Membrane completely flat; c) Membrane folded along one folding line. The torsional spring in the model simulates the membrane bending stiffness. .................................................................................................5

Fig. 40. a) the flexible model in three dimensions and inertial frame b) the model in $x-z$ view c) the model in $x-y$

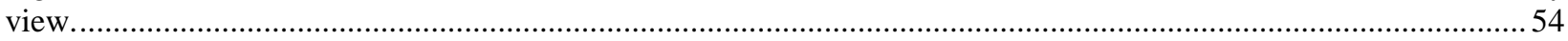

Fig. 41. Angles defining the direction of the imaginary rods with respect to an inertial frame. ...............................54 Fig. 42. Free body diagram of each lump mass a) $1^{\text {st }}$ free body diagram b) $2^{\text {nd }}$ free body diagram c) $3^{\text {rd }}$ free body

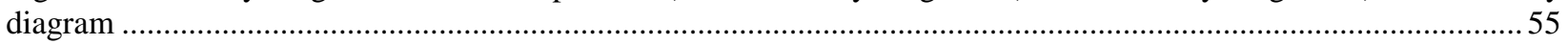

Fig. 43. Free body diagram of each lump mass a) Forces from torsional spring b) Forces from torsional damper ..... 56

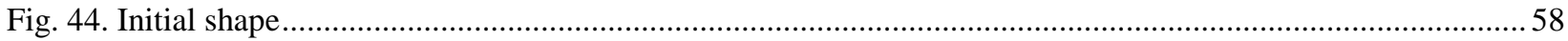

Fig. 45. The oscillation due to deformation from non-equilibrium results from torsional spring without external force

Fig. 46. Torsional spring and torsional damper test show that oscillate and back to equilibrium due to torsional

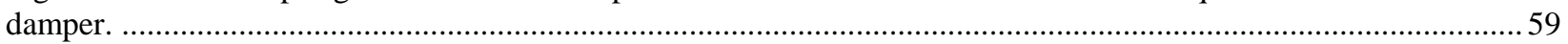

Fig. 47 Self-shadowing area simulation a) light source above the model and full shadow on the second plane b) shadow area after moving light source position c) shadow area after rotating. ......................................................... 60 Fig. 48 Comparison in eccentricity and inclination evolution of $f P E T_{j s}, f P E T_{j s, h}$ and $r P E T_{j s}$ under $\mathrm{J} 2$ and solar radiation pressure a) evolution over 12 days b) evolution over 0.5 days .................................................................. 62 Fig. 49. Comparison in eccentricity and inclination evolution of $f K a p_{j s}, f K a p_{j s, h}$ and $r K a p_{j s}$ under $\mathrm{J} 2$ and solar radiation pressure a) evolution over 12 days b) evolution over 0.5 day ...................................................................63 Fig. 50 Simulation results of PET under J2 and solar radiation pressure without self-shadowing in $0.320-0.325$ days (7.12 minutes) a) time-lapse of deformation b) absolute acceleration of both planes c) Euler angle evolution........... 64 Fig. 51 Simulation results of PET $^{\circledR}$ under J2 and solar radiation pressure with self-shadowing in 0.320-0.325 days (7.12 minutes) a) time-lapse of deformation b) absolute acceleration of both planes c) Euler angle evolution...........65 Fig. 52. Simulation results of Kapton ${ }^{\circledR}$ under J2 and solar radiation pressure without self-shadowing in 0.320-0.325 days (7.12 minutes) a) time-lapse of deformation b) absolute acceleration of both planes c) Euler angle evolution .. 66 Fig. 53 Simulation results of Kapton ${ }^{\circledR}$ under J2 and solar radiation pressure with self-shadowing in 0.320-0.325 days (7.12 minutes) a) time-lapse of deformation b) absolute acceleration of both planes c) Euler angle evolution........... 67 
Fig. 54 Comparison inclination and eccentricity evolution of $r P E T_{g}, f P E T_{g}, r K a p_{g}$ and $f K a p_{g}$ under $\mathrm{J} 2$ and third body perturbations over 12 days a) Inclination evolution b) Difference in inclination between flexible model and rigid model. c) Eccentricity evolution d) Difference in eccentricity between flexible model and rigid ..... .. 68

Fig. 55 Comparison in eccentricity and inclination evolution over 12 days of $f P E T_{j g, h}, f K a p_{j g, h}, r P E T_{j g}$ and $r K a p_{j g}$ under $\mathrm{J} 2$, third body perturbations and solar radiation pressure.

Fig. 56 Euler angle evolution fPET $_{\mathrm{jg}, \mathrm{h}}$ over 1days under $\mathrm{J} 2$, third body perturbations and the direct solar radiation pressure a) $1^{\text {st }}$ Euler angle b) $2^{\text {nd }}$ Euler angle c) $3^{\text {rd }}$ Euler angle......

Fig. 57 Euler angle evolution fKap $_{\mathrm{jg}, \mathrm{h}}$ over 1days under $\mathrm{J} 2$, third body perturbations and the direct solar radiation pressure a) $1^{\text {st }}$ Euler angle b) $2^{\text {nd }}$ Euler angle c) $3^{\text {rd }}$ Euler angle ................................................................... 70

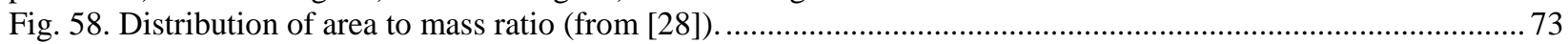

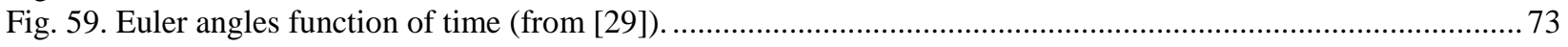

Fig. 60. Dependencies and interconnections among orbital, attitude and deformation dynamics......................... 74

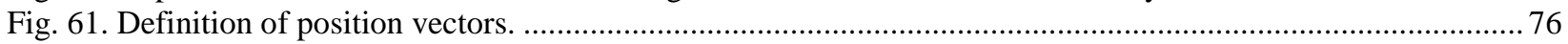

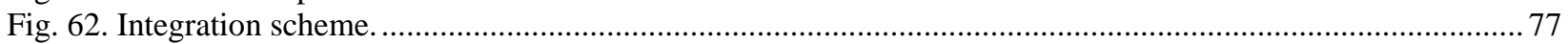

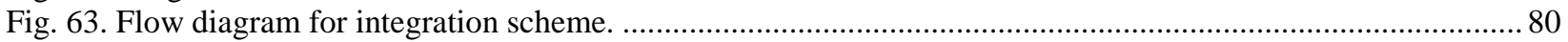

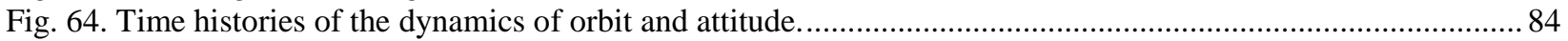

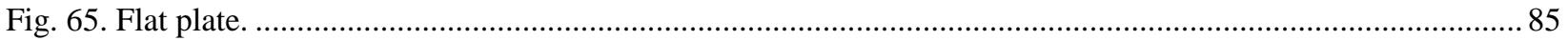

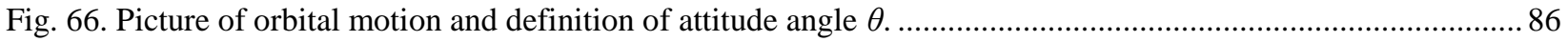

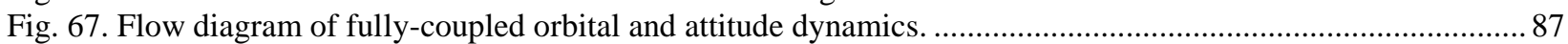

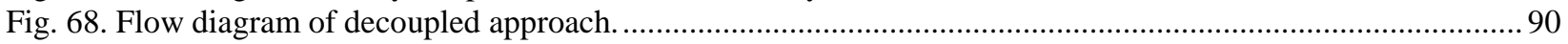

Fig. 69 Experimental set up for determination of MLI properties in a vacuum chamber. ....................................95

Fig. 70. Most element families currently available in commercial FEM software for mechanic analysis (from [52]) 97

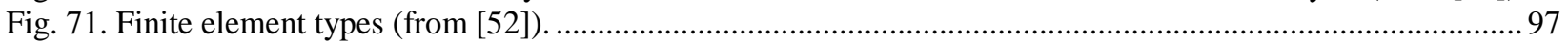

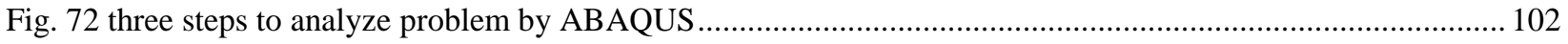

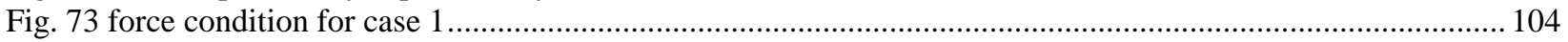

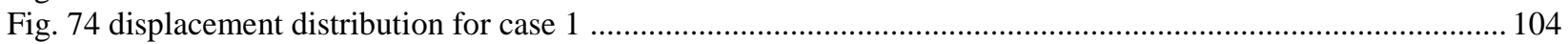

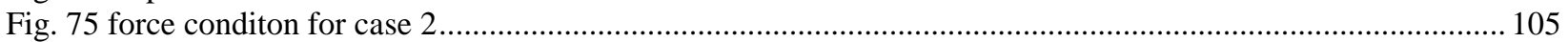

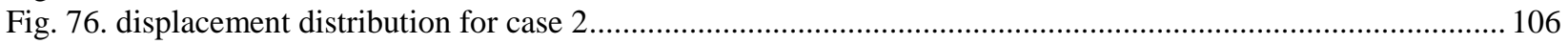

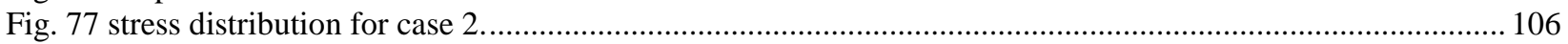

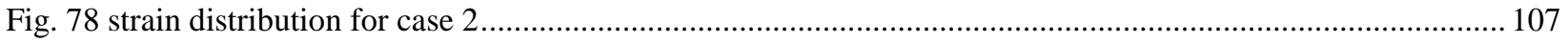

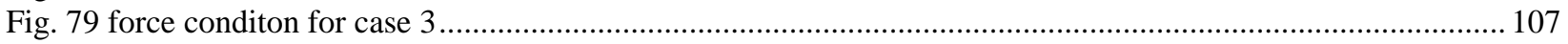

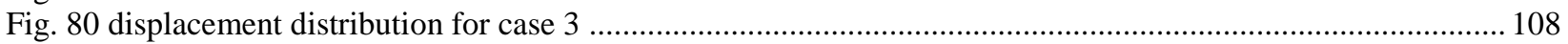

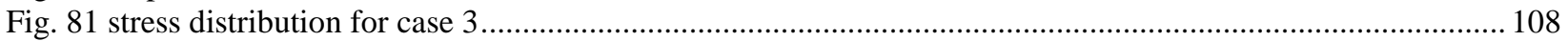

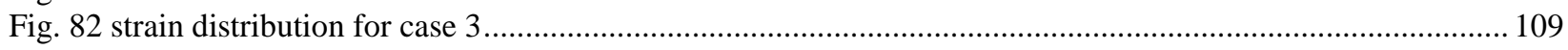

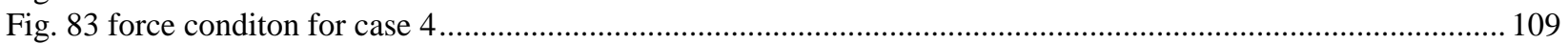

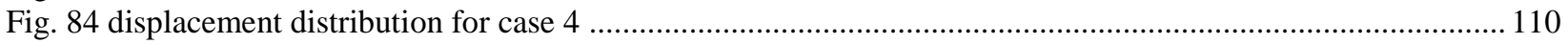

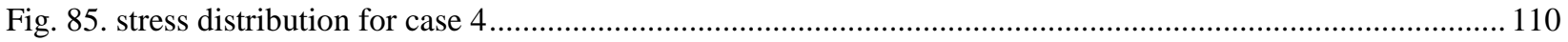

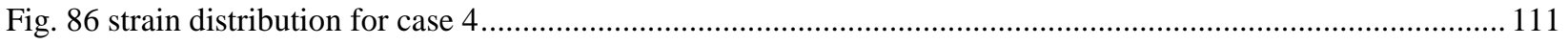

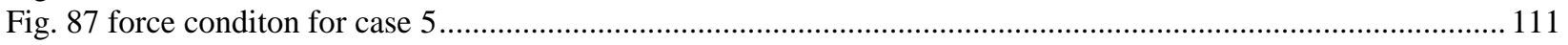

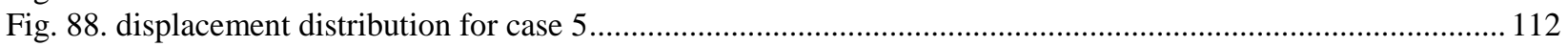

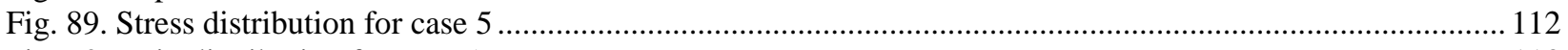

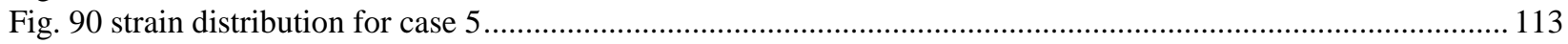

\section{List of Tables}

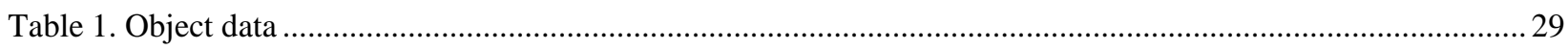

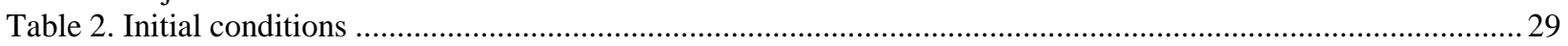

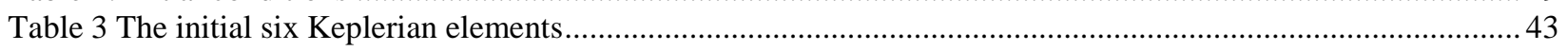

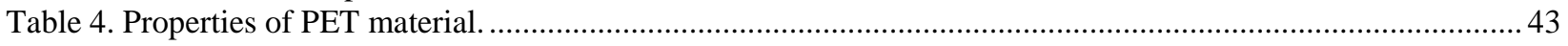

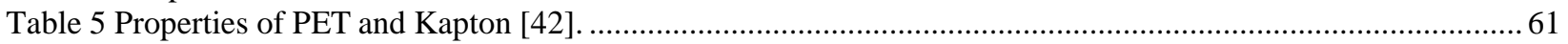

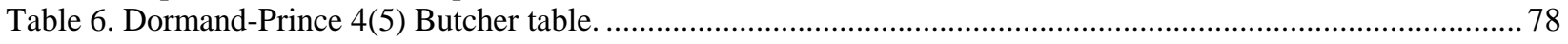

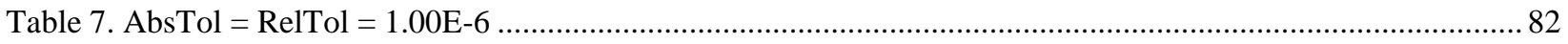

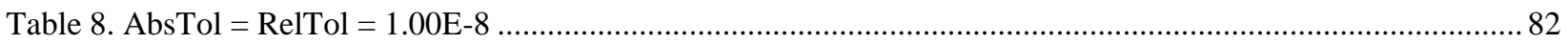

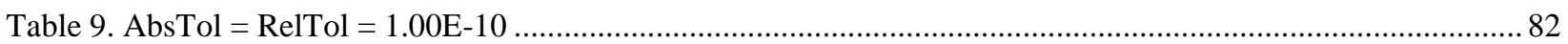




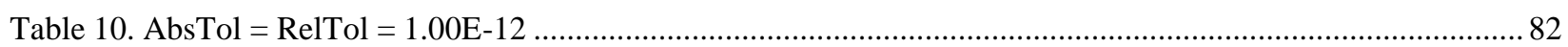

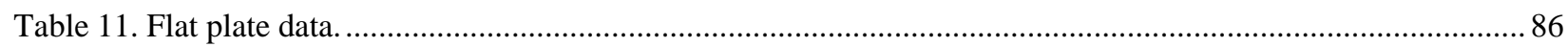

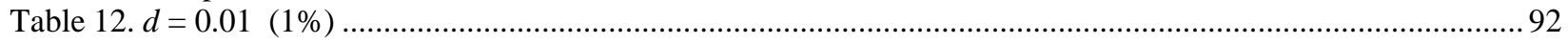

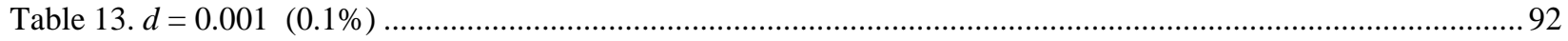

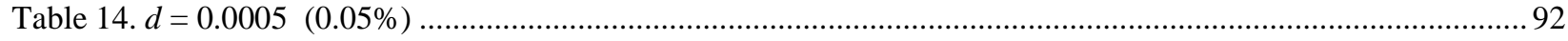

Table 15 properties and conditions for builing the model .................................................................. 103 


\section{Acknowledgements}

The following people contributed to the work presented in this report: Sittiporn Channumsin, Francesc Manresa Perez, Wang Xiaohan.

\section{Publications}

[1] S. Channumsin, M. Ceriotti, G. Radice, C. Frueh, "Orbital dynamics of lightweight flexible debris", in Proceedings of 64th International Astronautical Congress (IAC 2013), Beijing, China, 2013.

[2] S. Channumsin, M. Ceriotti, G. Radice, "A deformation model of flexible, high area-to-mass ratio debris under perturbations", in 1st Stardust Global Virtual Workshop, Glasgow, UK, 2014.

[3] S. Channumsin, M. Ceriotti, G. Radice, "A deformation model of flexible, high area-to-mass ratio debris for accurate propagation under perturbations", in Proceedings of 65th International Astronautical Congress (IAC 2014), Toronto, Canada, 2014. 


\section{Introduction}

Debris objects are uncontrolled, non-functional objects orbiting around the Earth. Natural debris includes asteroids and rocks, while artificial, human-made debris comprise non-active spacecraft (including launcher upper stages) and their components, fragmented following collision events or separation due to ageing. Debris objects often travel at very high velocities, and for this reason, despite their size can be relatively small, they that can threat active spacecraft, leading to catastrophic break-ups, which in turn generate new space debris. If the rate of production from collision is greater than decay rate (for example due to atmospheric reentry), then a chain reaction starts, which leads to the growth of the number of debris objects exponentially, as predicted by Kessler. This scenario is now known as "Kessler syndrome".

Since the early space era, the launch of Sputnik on 4 October 1957, more than 5,000 satellites and upper stages were launched between low Earth orbit (LEO) and geostationary Earth orbit (GEO) and led to increasing population of more than 22,000 trackable objects with sizes larger than $10 \mathrm{~cm}$. Approximately 1,000 of these are operational spacecraft. The remaining $94 \%$ are space debris, i.e defunct satellites and rocket upper stages which are not useful purpose. The routinely tracked objects (about 64\%) are fragments from some 250 breakups, mainly explosions and collisions of satellites or rocket bodies and space experiment. An estimated 700,000 objects larger than $1 \mathrm{~cm}$ and 170 million objects larger than $1 \mathrm{~mm}$ are expected to be in the Earth orbits. Fig. 1 (credit: NASA) shows a snapshot of known debris objects (not to scale) in LEO and GEO range.
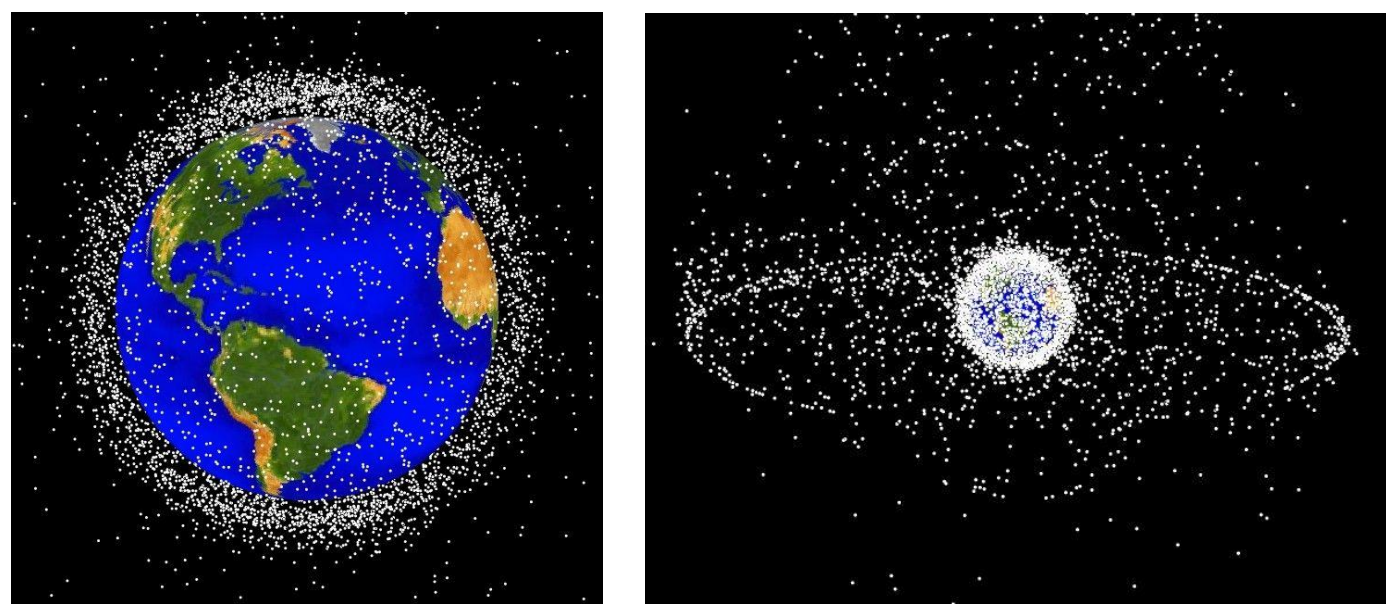

Fig. 1. Objects in Earth orbit that are currently being tracked. Approximately $95 \%$ of the objects in this illustration are orbital debris [Souce: NASA].

One of the most important debris-causing events in history happened on 11 January 2007. A direct-ascent, kinetic-kill anti-satellite (ASAT) vehicle was successfully tested by destroying an inactive Chinese Feng Yun 1C (FY-1C) weather satellite [1]. This event alone generated more debris objects than any previous space accident. The 
US Space Surveillance Network (SSN) was able to detect more than 900 pieces of debris that were at risk to damage operational spacecraft.

In February 10, 2009, defunct Russian military satellite Cosmos 2251 crashed the active U.S. communications satellite Iridium 33 at $790 \mathrm{~km}$ above Siberia [2]. This collision created two large debris clouds and the SSN reported that 382 pieces of debris from Iridium 33 and 893 pieces from Cosmos 2251 were created, and they will possibly remain in orbit for up to 10,000 years.

Fig. 2 shows some evidence of damage made by space debris to active spacecraft.
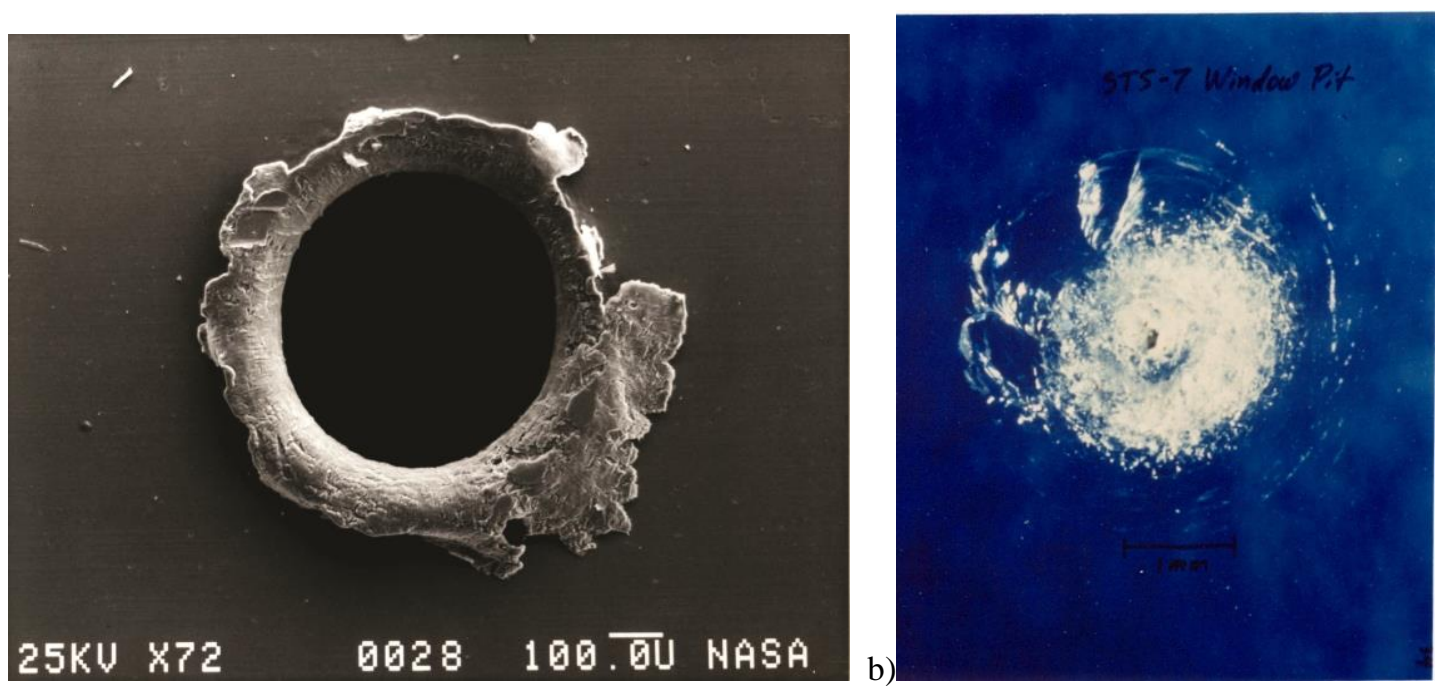

Fig. 2. (a) Hole made by debris in the panel of NASA's Solar Max experiment. (b) Window of the space shuttle damaged by a tiny piece of space junk (a paint fleck) during the STS-7 mission (Photo: NASA Orbital Debris Program Office)

\subsection{Survey on orbital debris and models}

\subsubsection{Fragmentation and distribution models}

Based on history of collision and explosions of spacecraft and upper stages and the observation data in low Earth orbit, the NASA Orbital Debris Program Office, established since the 1970s, studied and implemented a debris fragmentation distribution model which was implemented into EVOLVE. The model implements an estimation of size, area-to-mass and velocity of debris and the current version is EVOLVE 4.0.

Anz-Meador [3] validated debris sizes in the range $1 \mathrm{~mm}$ to $10 \mathrm{~cm}$, which pose major hazard to spacecraft. Both explosion models from EVOLVE and LEGEND were utilized to observe on-orbit fragmentation debris arising from the explosion of rocket bodies and payloads and ground-based laboratory tests. The data products for validation are from the US SSN, NASA Haystack and Auxiliary (HAX) radar observation campaigns and Satellite Orbital Debris Characterization Impact Test (SOCIT). It resulted that the number and size of the distribution is against the observed environment and conclusions were drawn that further tests were required before adopting the model. Instead, the area to mass distribution is adequate to describe the ballistic properties of both large and small debris objects. 
When the number of fragments resulting from a collision is of the order of hundreds or thousands, numerical simulations of their propagation become computationally very expensive, up to a point that they are not viable. For this reason, Li Yi-Yong [4] studied and developed novel approaches to propagate debris clouds accurately.

EVOLVE was originally capable to simulate the orbit debris in LEO, however it was expanded into GEO_EVOLVE 1.0 to simulate the orbital debris in the Earth geosynchronous region since 1999. The models were to be updated because of the overestimation of the future collision rate in GEO, due to variation of argument of perigee and precession of the nodes. The next update of GEO_EVOLVE 2.0 [5] improved two significant points: firstly, the development traffic database was upgraded with the inclusion of reliable insertion node, argument of perigee and mean anomaly of all launches. Secondly, perturbations were added, such as atmospheric drag, lunar gravitational perturbation, solar radiation pressure, Earth gravity field zonal $\left(\mathbf{J}_{2}, \mathbf{J}_{3}\right.$ and $\left.\mathbf{J}_{4}\right)$ and tesseral $\left(\mathbf{J}_{2,2}\right.$, $\mathbf{J}_{3,1}$, etc.) harmonics. GEOPROP and PROP3D propagators were used.

Another LEO-to-GEO evolutionary debris model, LEGEND [6], was developed by the NASA Debris Program Office at Johnson Space Center. It is based on historical satellite launch database (DBS-database) to reproduce the debris environment between 1957 and 2001 and predict future debris environment. It covers the near Earth space between 200 and 2,000 km, medium Earth orbit (MEO) and GEO (34,000-38,000 km) and super GEO (above 38,000 $\mathrm{km})$. This model provided all debris characteristics. The two propagators used in LEGEND were GEOPROP and PROP3D. The GEOPROP propagator calculated the motion of GEO objects while the PROP3D propagator focused on the orbits of all other satellites in the simulation.

All models are investigated from NASA, While the European Space Agency (ESA) started to develop the Meteoriod And Space Debris Terrestrial Environment Reference model (MASTER) in 1995 [7]. The model was introduced to derive spatial densities and velocity distributions in a 3D control volume from LEO and GEO altitude for the debris size larger than $1 \mathrm{~mm}$, similarly to NASA's EVOLVE and LEGEND. MASTER 2009 added data from new collision and observations by the TIRA, EISCAT, Goldstone and Haystack radars and the ESA Space Debris Telescope (ESA-SDT). High area-to-mass ratio (HAMR) objects were also considered [8]. According to the observed data by ESA space debris telescope to characterize the space debris environment near the geostationary ring [9], there were 2,790 uncorrelated targets brighter than magnitude 18.5. A Gaussian distribution was applied to approximate the number of detections, focusing on objects with magnitude higher than 18.5 with a probability about 95\%. The study determined detection probabilities for a given field of view (FOV) and compared the ESA data: the results were in good agreement with the debris population models of MASTER.

\subsubsection{Debris in GTO}

In geosynchronous transfer orbits (GTO), debris are mainly created by disposed rocket upper stages and fragments from rocket explosions and collisions [10]. Most of GTOs perigees are in LEO, below $2000 \mathrm{~km}$, and apogees near or above GEO. Therefore, debris in GTO has the potential for interfering with objects in both LEO and GEO, which is the reason why strategies and approaches were suggested to minimize space debris in GTO [11], like re-entry at perigee in LEO. In a study of the probability of collision (PC) of spacecraft in GEO, Mcknight [12] 
considered parameters as spatial density (SPD), relative collision velocity (VR), collision cross-section of satellite at risk $(\mathrm{AC})$ and time $(\mathrm{T})$.

\subsubsection{High area-to-mass ratio debris}

The observation by Schildknecht [13] with the ESA 1-metre telescope (ESASDT) [14] and Zimmerwald Laser and Astrometry Telescope (ZIMLAT) revealed small-size debris objects in highly elliptical orbits. It found the new type of debris population for 7 unknown which was small size of debris in elliptical orbits. Three of the identified objects orbited in a classical GTO with eccentricity about 0.7. Other four, instead, had semi-major axes (and hence period) close to that of the GEO [15], but the range of eccentricities was between 0.13 and 0.49 . Subsequently, around 80 objects of the new population were found but the original sources were unknown, until it was assumed that they were pieces of multi-layer insulation (MLI) material. This material is very lightweight (high area-to-mass ratio) and highly reflective: the solar radiation pressure significantly perturbs HAMR objects, resulting in periodical variations of eccentricity and inclination. In that work, the numerical integrator SATORB was applied to with a force model including the geopotential harmonics up order twelve. Other perturbations included the SRP, the solar and lunar gravitational forces and the Earth's shadow effects. Lastly, the objects were propagated assuming random tumbling motion and shapes. The light curves showed a wide variety of signatures, ranging from periodic or random variations of several magnitudes over time spans of a few minutes to constant brightness over 10 min. It was also observed degradation of FEP material properties of the MLI around the telescope Hubble space telescope and this material was returned to Earth for deep investigation [16]. Today, there is suspect that this type of debris is made of multi-layer insulation, but there is no evidence to confirm so.

Further experiments were conducted by Murakami et al. to investigate fragmentation of multi-layer insulation [17], and results were compared with NASA breakup model, showing similar trend in term of mass distribution. Some evidence supports that the mass distribution concerning material in the experiments of the study of Murakami [17] and support that most of High-Area-mass-ratio were MLI that was one of debris in space. Anselmo et al. [18] investigated the long-term evolution of HAMR debris, showing variation of inclination and eccentricity over centuries.

A novel approach to determine the average cross-sectional area of breakup fragments was proposed by Hanada et al. [19]. This approach provides a good estimate in the calculation of the cross-sectional area of multi-layer insulation fragments.

\subsubsection{Effect of SRP}

Light curves are often used to determine the properties of debris objects. There are however unresolved light curves of objects orbiting in GEO, which could be matched with MLI-type of objects. The investigation in [20] defined probable initial conditions of angular momentum, torque, quaternion representation of orientation. Linear combinations of the database were considered to model objects with both specular and diffuse reflection. Furthermore, angular momentum and torque were calculated using solar radiation pressure considering specular and 
diffuse reflection. The three types of MLI were investigated, and according to the study, the angular momentum depended on the area-to-mass ratio, the irregularity of shape reflectivity and the internal friction.

Flegel [21] investigated MLI and rocket upper stages and parts of satellites (LMRO) in term of total spatial object density on altitude and over time from 1958 to 2010. The number of MLI objects peaked around 900-1400 km and decreased slowly in GEO while LMRO population distribution is peak in range between 1,000 and 5,000. In the GEO region, the peak of number LMRO was at $25,000 \mathrm{~km}$ and $36,000 \mathrm{~km}$. The number of MLI-type objects was higher than that of LMRO. The model was validated comparing the observation data from ESA's PROOF (Program for Radar and Optical Observation Forcasting).

Liou et al. [23] proposed that the HARM could be a plausible explanation for the debris with highly eccentric orbits with period similar to GEO. The global behavior of thousands of HAMR debris objects revealed the unique pattern of their distribution in space.

The orbit determination of high area-to-mass ratio objects in GEO trajectories was analyzed by Kelecy et al. [22]: the cooler cover of a MSG-2 satellite was used for the investigation and the effects of the non-modeled accelerations was considered to predict the HAMR object trajectory. The analyzed objects were assumed to be flat plates, covered with MLI. The simulator included Earth gravity zonal harmonics, luni-solar gravitational perturbations and attitude dependent solar radiation pressure acceleration that considered reflective, diffusive and absorption components. An extended Kalman filter (EKF) was used to estimate the orbit in the orbit determination tool kit (ODTK). The initial position and velocity states were derived using a combination of Gauss-Gooding initial orbit determination (IOD).

Kelecy et al. [23] studied the solar radiation pressure effect on a variety of high area-to-mass ratio debris in GEO orbit by focusing on MLI or thermal insulation, to improve the orbit predictions. It was found that the characteristics and dynamics of the objects could be identified by tracking and measuring data.

A semi-analytical theory was used to analyze the gravitational perturbation of debris in GEO by Valk et al. [24], who also considered the Earth's shadowing effects on HAMR objects in the geosynchronous region. Because of the non-singular set of equations used, the method was particularly suitable for both near circular and near equatorial orbits as well as orbits that transit periodically around null eccentricities and null inclinations. The short, mid and long term propagation under SRP and the shadowing effects was applied to objects of $20 \mathrm{~m}^{2} / \mathrm{kg}$ and the numerical and semi-analytical methods were compared over different time scales. Inclination, eccentricity and semi-major axis were used to assess the results of the propagations.

Another investigation of the shadow effects on high area-to-mass geostationary space debris, in the short and long term time evolution, was done by Hubaux [25]. The mean exponential growth factor of nearby orbits (MEGNO) was introduced to investigate both shadow conical and cylindrical Earth's shadowing model. It resulted that the cylindrical model was unreliable for predicting the orbital behavior, while one of conical modelled was proven to be better because of its realistic umbra-penumbra transition model.

MEGNO was applied by Valk [26] to propagate the dynamics of HAMR in GEO in the long term. The validation of the method was by propagation on over 12,600 orbits and over 30 years of time span. Perturbations were considered such as the influence of the Earth's gravity field, both the gravitational perturbation of the sun and the moon, and the direct solar radiation pressure. A sensitivity analysis to initial conditions was done. Secondary 
resonances on both sides of the well-known pendulum like the pattern of geostationary objects were found. In this work, MEGNO proved to be an efficient method to propagate the phase space.

Another study in long-term dynamics of HAMR debris is in [18]: a range of objects, including and multi-layer insulation (MLI) blankets and other low density composite material, were considered. The selected initial conditions were that of objects released in geosynchronous orbit, and a high-accuracy numerical integrator was used, which included the zonal and tesseral harmonics of the Earth, shadow effects and atmospheric drag. Interestingly, the dimensionless radiation pressure coefficient $C_{r}$ was considered for taking into account the deformed geometry of debris, affecting the area to mass ratio, such that $C_{r} \mathrm{x} A / m$ gives the effective area-to-mass ratio exposed to the SRP.

The resonance effect caused by the geometry of the Earth-Moon-Sun systems in case of HAMR objects was also studied by Rosengren [27]. It was noted that the mean geometry of the Earth-Moon-Sun system repeats itself closely after a period of time, of about 223 synodic months, resulting in a dynamical system moving in a nearly periodic orbit. When displayed in the heliocentric orbit frame, the actual motion resulted very complicate.

In many studies, pieces of multi-layer insulation blankets are suspected to be HARM debris but there is no observational proof of this. Most "classical" low AMR GEO debris objects have flat or slowly varying light curves, instead HAMR objects show all variations with period of a few minutes and shorter. One of the issues is that the knowledge of the surface properties and the shapes of the objects including specular and diffuse reflection, albedo, and color are required to identify these types of debris.

The observation of HAMR objects from ESA Space Debris Telescope (ESASDT) was used for the study by Früh et al. [28], who investigated the variation of their area-to-mass ratio and orbital parameters and showed that shadowing effects were significant influence on the long term evolution of the orbits. However, in this work, the AMR values were assumed to be constant: the results were not the exact dynamics of HAMR objects because effects due to irregular shape, complex attitude motion and deformation of the actual objects were not considered. These effects produce an actual change of the AMR value over time.

More recently, a further investigation of MLI debris in near geosynchronous orbits was done by Früh, [29]. Here, MLI objects were assumed to be flat plates with material properties of PET and Kapton, as these materials are those mostly used to protect spacecraft. Propagation considered gravitational field, direct solar radiation pressure, Earth magnetic field and the influence of shadow effect with a simple cylindrical shadow model. An important point of this study is that it coupled both orbit and attitude propagation over 4 days. Then, light curve were used to correlate the attitude. The results demonstrated that the solar radiation torque and gravitational torque led to rapid attitude motion, while magnetic torques stabilize the attitude motion preventing a fast spin. Resulting overestimation of the evolution of eccentricity and inclinations originated from the assumption of a constant AMR value.

Another study of the effect of SRP on orbital debris is that of Früh and Schildknecht [28]. The work used several objects, including an upper stage rocket debris, Block D-2, simplified in a cylindrical shape, and other HAMR objects modelled as flat rigid sheets, which were tracked over a number of years. The attitude motion was correlated with the observed light curves, which measured the variation of the brightness of the object due to lighting condition, viewing geometry, shape, reflection properties and attitude motion. The considered perturbations were the third body gravitational field and solar radiation pressure. The orbit attitude motion was calculated by Euler's equation using the assumption of debris as rigid body. The study showed that the flat sheet was subject to fast oscillations, while the 
attitude motion of the upper stage was significantly influenced by an offset between geometrical center and the center of mass object.

The study of the evolution of the relative velocity and the eccentricity of particles due to SRP was done by Celestino et al. [10]. Different particle sizes were considered: according to the results, particles size less than $5 \mu \mathrm{m}$ were significantly affected by SRP. These sizes of debris experienced a very large amplitude of oscillation in the eccentricity value over time, leading to a natural mechanism of removal when passing close the region of influence of air drag. Instead, the SRP affected the larger debris particles in the short period inducing changes in eccentricity and semi-major axis. 


\section{Dynamics}

\subsection{Orbital dynamics}

\subsubsection{Orbital elements}

Orbital elements are the parameters to identify a specific orbit around the Earth. The common sets usually are classical orbital elements, or Keplerian elements illustrated in Fig. 3.

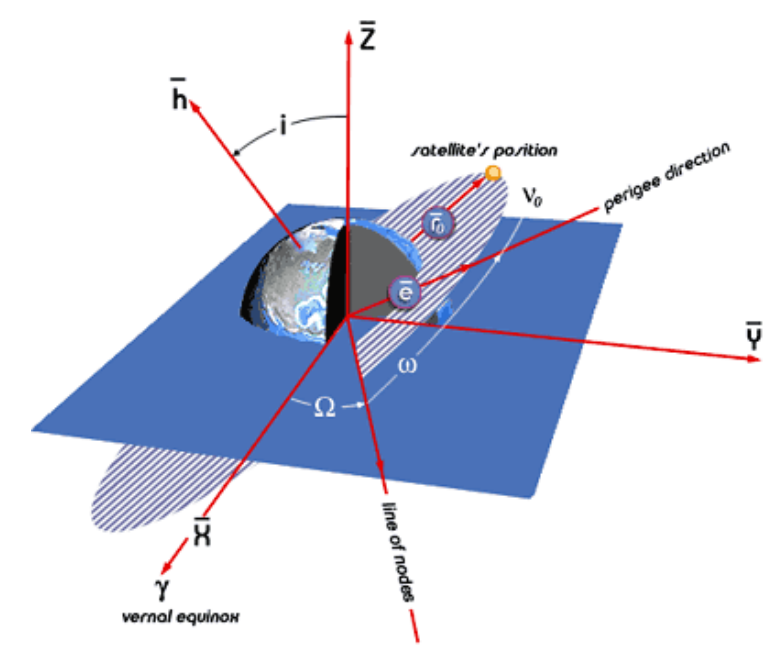

Fig. 3. Orbital elements.

\subsubsection{Dimensional Elements}

These describe the shape and size of the orbit and relate the position on orbit:

- $A$ is the semi-major axis of the orbit, identifying its size.

- $E$ is the eccentricity of the orbit determining how elliptical the orbit is.

- $t$ is the time of the satellite measured from the periapsis passage (closest approach to the main attractor). It relates to the mean motion, $M$. It determines the satellite's current position. Alternatively, the true anomaly $\theta$ can be used.

\subsubsection{Orientation Elements}

- $\quad i$ is the inclination, i.e. the angle between the orbit plane and respect to the reference plane. If $0^{\circ}<i<$ $90^{\circ}$, the motion is termed as direct or prograde and retrograde if $90^{\circ}<i<180^{\circ}$.

- $\omega$ is the argument of perigee, i.e. is the angle from the ascending node to the eccentricity vector, measured anticlockwise.

- $\Omega$ is the right ascension of the ascending node, or RAAN, the angle from the direction of the vernal equinox to the line of the nodes, measured counterclockwise. 
The six classical orbital elements are useful and intuitive to describe the orbit and current position of satellites. However, the equations to describe the dynamics are highly nonlinear.

\subsubsection{Coordinate systems}

\subsubsection{Earth-centered inertial (ECI) coordinate system}

The origin is located in the center of the Earth, and the three axes point into inertial directions. The $\mathrm{z}$ axis points towards the North Pole and it is perpendicular to the equatorial plane. The $\mathrm{x}$ axis points towards the vernal equinox (first point of Aries), and the y axis completes the right handed frame.

\subsubsection{Earth-centered Earth-fixed (ECEF) coordinate system}

The origin is located in the center of the Earth, and the three axes are fixed to the Earth. The $\mathrm{z}$ axis points towards the North Pole and it is perpendicular to the equatorial plane. The $\mathrm{x}$ axis points towards the Greenwich meridian, and the y axis completes the right handed frame. This frame is used to describe the position of terrestrial. Fig. 4 shows the both coordinate systems.

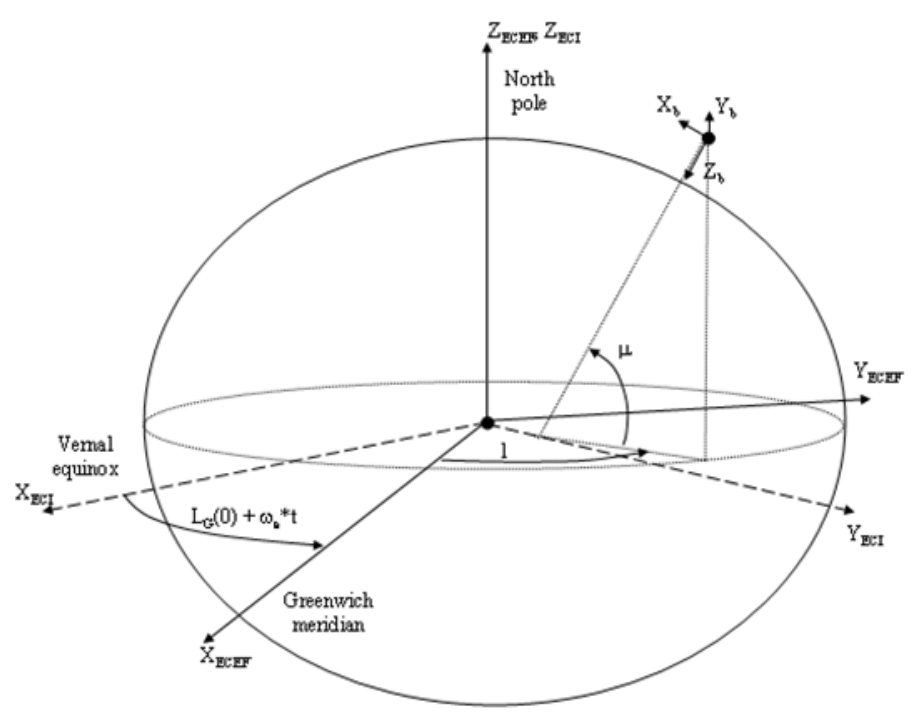

Fig. 4. ECI and ECEF coordinate systems.

\subsubsection{Two-body problem}

Newton's law of universal gravitation states that every point mass attracts every single point mass by a force pointing towards the other mass and proportional to the product of the two masses and inversely proportional to the square of the distance between them. In this research, we focus on a body (debris), of mass $m$, orbiting around the Earth, whose mass is $m_{\oplus}$ :

$$
\mathbf{F}=-G \frac{m m_{\oplus}}{r^{2}} \hat{\mathbf{r}}
$$

where $G$ is the universal gravitation constant, $6.674 \times 10^{-11} \mathrm{Nm}^{2} \mathrm{~kg}^{-2}$, and $\mathbf{r}$ is the position vector of the object from the origin in the center of the Earth. 


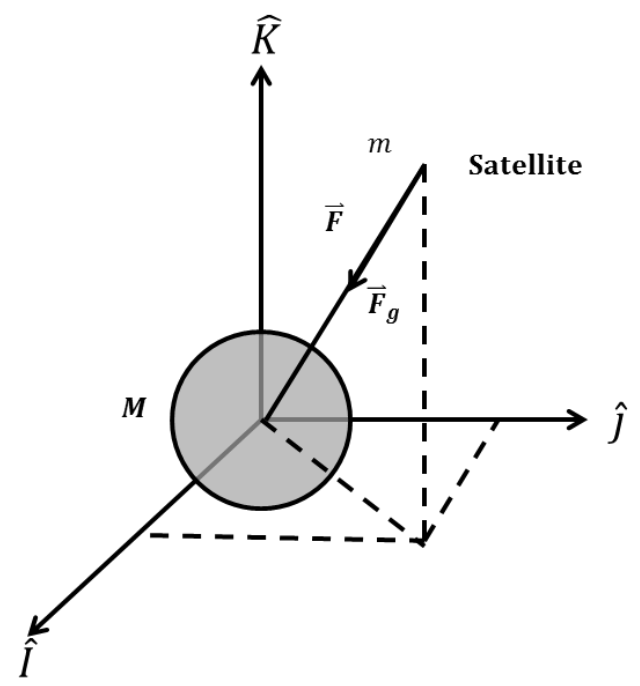

Fig. 5. Gravitational force from the Earth acting on a satellite

By introducing the approximation that the orbiting mass is much smaller than that of the Earth, it is possible to obtain the following differential equation of motion for the object with respect to the center of the Earth:

$$
\ddot{\mathbf{r}}=-\frac{\mu}{r^{2}} \hat{\mathbf{r}}
$$

where $\mu=G\left(m_{\oplus}+m\right) \approx G m_{\oplus}$.

\subsubsection{Third body perturbations}

For an object orbiting the Earth, the effects of gravitational attraction from other (third) bodies, such as the Sun and Moon become more significant with the orbit altitude. In geosynchronous orbit, the main third body gravitational perturbing forces acting on the objects are the Sun and Moon. The equation of motion, including the gravitational perturbation of $N$ other bodies (as derived in [30]) is:

$$
\ddot{\mathbf{r}}=G m_{\oplus}\left(\frac{\mathbf{r}}{r^{3}}\right)+G \sum_{k=1}^{N} m_{k}\left\lfloor\frac{\mathbf{r}_{k}-\mathbf{r}}{\left|\mathbf{r}_{k}-\mathbf{r}\right|^{3}}-\frac{\mathbf{r}_{k}}{r_{k}^{3}}\right\rfloor
$$

where $\mathbf{r}_{k}$ is the position vector of the k-th attractor (Sun, Moon) and $m_{k}$ its mass.

\subsubsection{Solar radiation pressure}

The solar radiation pressure (SRP) is caused by the incident rays of light (photons) from the Sun on the exposed surfaces of the object. It is a non-conservative and non-gravitational disturbance. This perturbation in GEO is usually dominant, especially for objects which have large surfaces exposed to the sun, and small masses (i.e. high area-tomass ratio). Modelling the SRP perturbing acceleration is difficult for a number of reasons.

First of all, the solar flux generally varies depending on the time of the year and the solar activity, which has its own cycle. In addition, for an accurate model of the acceleration, all the areas exposed to the sun have to be considered. This includes modelling the Earth eclipses during the orbit. Finally, the reflective properties of the 
materials on the surfaces exposed to the sun are also important to determine the force applied to each one of them. The development of an accurate model concerns the effectiveness to calculate the position of the sun with respect to the object and the Earth at any given time during the orbit as well as the correct attitude of the objects.

According to Wertz [31], solar flux value is measured at Earth aphelion point is:

$$
S F=\frac{1358}{1.004+0.0334 \cos D_{\text {aphelion }}} \mathrm{W} / \mathrm{m}^{2}
$$

where $D_{\text {aphelion }}$ is defined as $2 \pi$ times the time elapsed from Earth aphelion, expressed as a fraction of the whole year.

The amount of momentum that is carried by this flux can be found through Einstein's law, $E=m c^{2}$, and can be expressed as a force per unit area, or pressure, as

$$
p_{s r}=\frac{S F}{c} \cong 4.57 \times 10^{-6} \frac{N}{m^{2}}
$$

Where $\mathrm{c}$ is the speed of light. The numerical value is approximate at 1 Astronomical Unit (AU).

The actual value and direction of the force experienced by a flat surface subject to $p_{s r}$ depends on the orientation of the surface with respect to the sun and the reflectivity properties of the surface. Fig. 6 shows the effect of specular reflection of the photons and diffuse reflection, typical of a real, rough surface. In addition, Lambertian diffusion is considered the diffuse and specular radiation forces that affect to the object and consider each object surface area as following. In the ideal case of pure specular reflection, the force applied is perpendicular to the surface. In non-ideal cases, the force is deflected by a certain angle, as diffuse reflection and Lambertian diffusion generate tangential forces (see Fig. 7 for reflection geometry). The same figure shows the incident angle, defined as the angle between the normal unit vector to the surface and the unit vector defining the direction from the Sun to the surface. The reflected angle is equal to the incident in the case of purely specular reflection.

The acceleration due to SRP can be computed, considering the summation over each flat surface $i$ exposed to the sun, as:

$$
\mathbf{a}_{r a d}=\sum_{i=1}^{N} \frac{p_{s r} A_{i} \cos \phi_{i n c, i}}{m}\left\{2\left(\frac{C_{R d_{i}}}{3}+C_{R s_{i}} \cos \left(\phi_{i n c, i}\right)\right) \hat{\mathbf{n}}+\left(1-C_{R s_{i}}\right) \hat{\mathbf{s}}\right\}
$$

where $A_{i}$ is the area of the $\mathrm{i}$-th surface, $m$ is the mass of the entire object, and $C_{r d}$ and $C_{r s}$ are the coefficients of diffuse and specular reflectivity, respectively [30]. If we consider that the surface can also absorb a fraction of the photons, given by a coefficient $C_{r a}$, then it must be $C_{r s}+C_{d s}+C_{r a}=1$. The surface normal unit vector, $\hat{\mathbf{n}}$, and the sun vector $\hat{\mathbf{s}}$ are required to be known, from the orientation of the specify the orientation of the object. The summation adds the $N$ flat plate surfaces of the satellite model. 


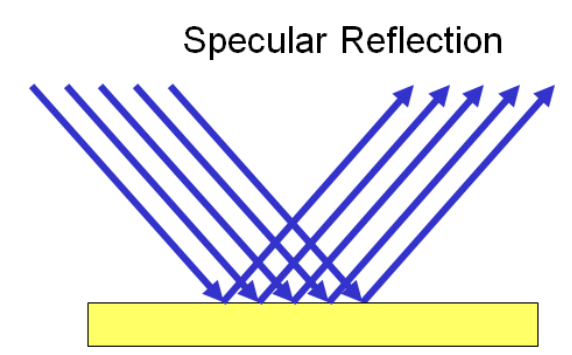

Fig. 6. Specular and diffuse reflection.

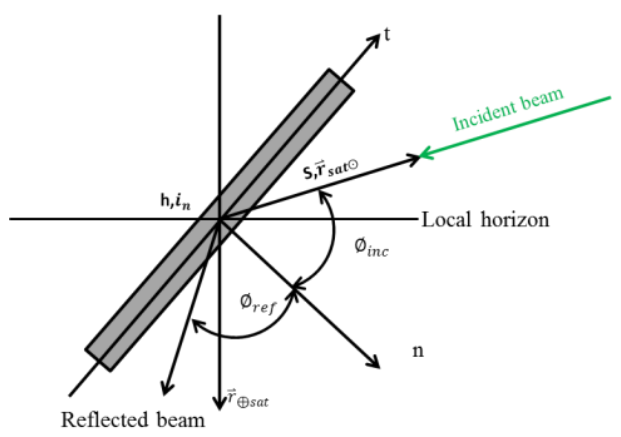

Fig. 7. Incident solar radiation geometry. Note that the unit vectors $t$ and $\mathbf{n}$ are the unit vectors in the tangential and normal directions (with respect to the surface), respectively; $h$ and in are oriented upwards from the plane of the paper; $s$ and $r_{\text {sat } \odot}$ are collinear with the incident solar rays, pointing towards the sun.

\subsubsection{Shadow effect}

The shadow effect is a partial or total obscuration of the sunlight from the satellite by the Earth. A simplified cylindrical Earth shadow model, as that in Fig. 8, does not consider penumbra: the object is either in sunlight or complete shadow. A conical model, instead, uses a more realistic conical Earth shadow with two main regions, penumbra and umbra as in Fig. 9. In the penumbra region, the sun is partially eclipsed from the Earth. Clearly, the solar radiation pressure is zero completely when satellite is in the Earth shadow. It is instead more complex to find the SRP value in the penumbra region, and this limits the applicability of this model.

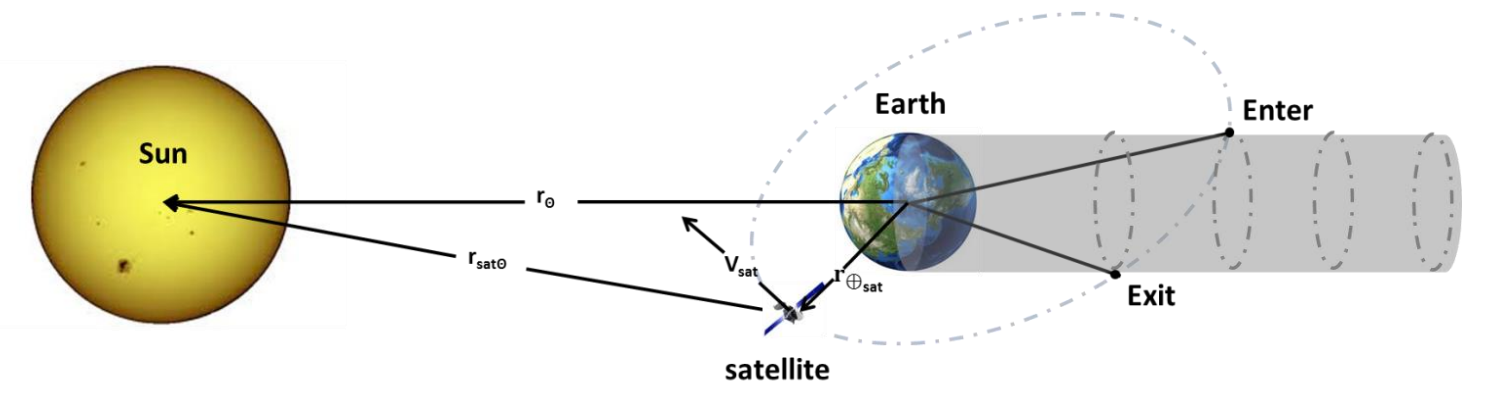

Fig. 8. Cylindrical eclipse geometry. 


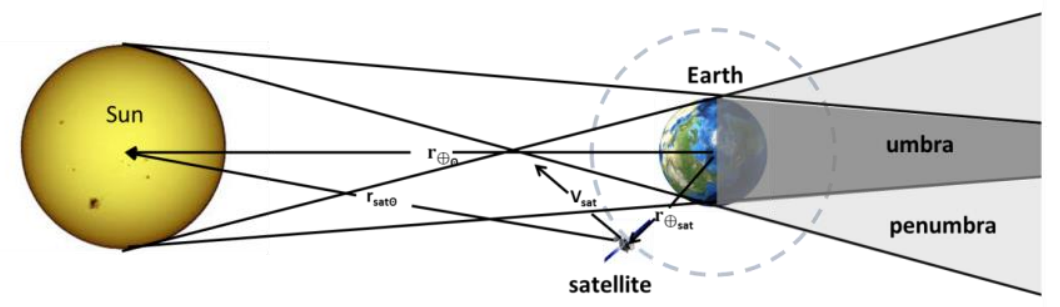

Fig. 9. Eclipse geometry for conical model.

In this work, we will the cylindrical model. Whether the spacecraft is in umbra or sunlight can be computed according to Vallado [30] through the following parameters:

$$
\begin{gathered}
\tau_{\text {min }}=\frac{\left|\mathbf{r}_{1}\right|^{2}-\mathbf{r}_{1} \cdot \mathbf{r}_{2}}{\left|\mathbf{r}_{1}\right|^{2}+\left|\mathbf{r}_{2}\right|^{2}-2 \mathbf{r}_{1} \cdot \mathbf{r}_{2}} \\
\left|\mathbf{c} \tau_{\text {min }}\right|^{2}=\frac{\left(1-\tau_{\text {min }}\right)\left|\mathbf{r}_{1}\right|^{2}+\left(\mathbf{r}_{1} \cdot \mathbf{r}_{2}\right) \tau_{\text {min }}}{R_{\oplus}^{2}}
\end{gathered}
$$

where $R_{\oplus}=6,371 \mathrm{~km}$ is the mean radius of the Earth. Then,

- If $\tau_{\min }<0 \vee \tau_{\min }>0 \vee\left|\mathbf{c} \tau_{\min }\right|^{2}>1$, the object is in sunlight;

- Else, the object is in umbra.

The SRP acceleration is simply set to zero when the object is in umbra, otherwise the actual value is used, as computed in the previous section.

\subsubsection{Sun vector}

The unit vector $\hat{s}$ defines the direction from satellite to the Sun at any given time as:

$$
\hat{\mathbf{s}}=\frac{\mathbf{r}_{s a t} \odot}{\left|\mathbf{r}_{s a t}\right|}
$$

where $\mathbf{r}_{s a t} \odot$ is the vector from the satellite to the Sun as in Fig. 10, as:

$$
\mathbf{r}_{s a t} \odot=\mathbf{r}_{\oplus \odot}-\mathbf{r}
$$

According to Vallado [30], the Earth-Sun vector $\mathbf{r}_{\oplus \circ}$ is calculated at any given time by using the following formulas. First, we define the number of Julian centuries as a function of the Julian Date $J D$ :

$$
T_{T D B}=\frac{J D-2451545}{36525}
$$

Then the mean longitude $\lambda_{M \oplus}$, the solar mean anomaly $M_{\odot}$, the ecliptic longitude $\lambda_{\text {eclipic }}$, the distance of the sun with respect to the Earth $r_{\odot}$, and the obliquity of the ecliptic, $\varepsilon$, from the following equations: 


$$
\begin{gathered}
M_{\odot}=357.5277233^{\circ}+35999.05034 T_{T D B} \\
\lambda_{M \odot}=280.460^{\circ}+36000.770 T_{T D B} \\
\lambda_{e c l}=\lambda_{M}+1.914666471 \sin \left(M_{\odot}\right)+0.019994643 \sin \left(2 M_{\odot}\right) \\
r_{\odot}=1.000140612-0.0167086 \cos \left(M_{\odot}\right)-0.000139589 \cos \left(2 M_{\odot}\right) \\
\varepsilon=23.439291^{\circ}-0.0130042 T_{T D B}-1.64 \times 10^{-7} T_{T D B}^{2}+5.04 \times 10^{-7} T_{T D B}^{3}
\end{gathered}
$$

Given $\lambda_{\text {ecliptic }}$ and $\varepsilon$ at epoch, the sun position vector with respect to the Earth is:

$$
\mathbf{r}_{\odot}=\left[\begin{array}{c}
r_{\odot} \cos \left(\lambda_{\text {ecliptic }}\right) \\
r_{\odot} \cos (\varepsilon) \sin \left(\lambda_{\text {ecliptic }}\right) \\
r_{\odot} \sin (\varepsilon) \sin \left(\lambda_{\text {ecliptic }}\right)
\end{array}\right\}
$$

where all distances are in AU.

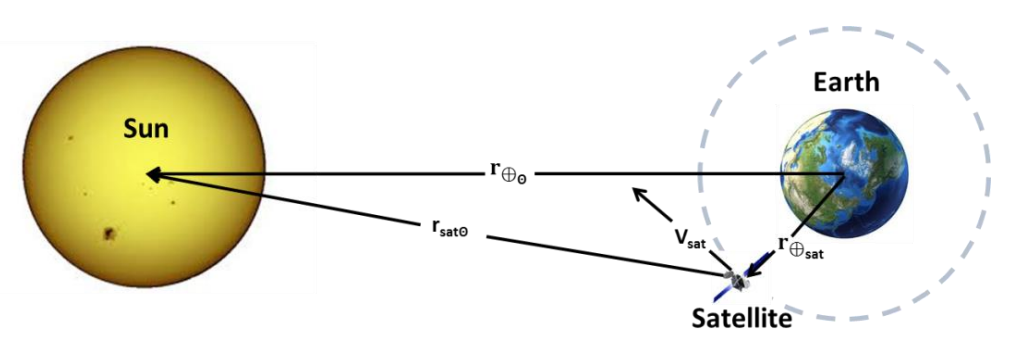

Fig. 10. Earth-Satellite-Sun vector diagram

\subsubsection{Moon vector}

The Moon position with respect to the Earth is computed in a similar way [30]:

$$
\begin{aligned}
& \lambda_{\text {ecliptic }}= 218.32^{\circ}+481,267.883 T_{T D B}+6.29 \sin \left(134.9+477,198.85 T_{T D B}\right)- \\
& 1.27 \sin \left(259.2-413,335.38 T_{T D B}\right)+0.66 \sin \left(235.7+890,534.23 T_{T D B}\right)+ \\
& 0.21 \sin \left(269.9+954,397.70 T_{T D B}\right)-0.19 \sin \left(357.5+35,999.05 T_{T D B}\right)- \\
& 0.11 \sin \left(186.6+966,404.05 T_{T D B}\right) \\
& \phi_{\text {ecliptic }}= 5.13^{\circ}\left(93.3+481,202.03 T_{T D B}\right)+0.23 \sin \left(228.2+960,, 400.87 T_{T D B}\right)- \\
& 0.28 \sin \left(318.3+6003.18 T_{T D B}\right)-0.17 \sin \left(217.6+407,332.20 T_{T D B}\right) \\
& \rho=0.9508^{\circ}+0.0518 \sin \left(134.9+477,198.85 T_{T D B}\right)+ \\
& 0.0095 \cos \left(259.2+413,335.18 T_{T D B}\right)+0.0078 \cos \left(235.7+890,534.23 T_{T D B}\right)+ \\
& 0.0028 \sin \left(269.9+954,397.70 T_{T D B}\right) \\
& r_{M \text { oon }}=\frac{1}{\sin \rho}
\end{aligned}
$$


where $\lambda_{\text {ecliptic }}, \phi_{\text {ecliptic }}$ are the ecliptic longitude and latitude of the Moon respectively, $\rho$ is the horizontal parallax, and $r_{\text {Moon }}$ is the distance of the Moon from the Earth. Finally,

$$
\mathbf{r}_{\text {Moon }}=r_{\text {Moon }}\left\{\begin{array}{c}
\cos \left(\phi_{\text {ecliptic }}\right) \cos \left(\lambda_{\text {ecliptic }}\right) \\
\cos (\varepsilon) \cos \left(\phi_{\text {ecliptic }}\right) \cos \left(\lambda_{\text {ecliptic }}\right)-\sin (\varepsilon) \sin \left(\phi_{\text {ecliptic }}\right) \\
\sin (\varepsilon) \cos \left(\phi_{\text {ecliptic }}\right) \cos \left(\lambda_{\text {ecliptic }}\right)+\cos (\varepsilon) \sin \left(\phi_{\text {ecliptic }}\right)
\end{array}\right]
$$

\subsection{Attitude dynamics}

The angular momentum $\mathbf{H}$ of a rigid body with respect to its center of mass is defined as by the following equation:

$$
\mathbf{h}=\int \mathbf{r} \times(\mathbf{r} \times \boldsymbol{\omega}) d m
$$

$\mathbf{r}$ denotes the location vector of a particle mass element $d m$ and $\boldsymbol{\omega}$ is the angular velocity vector of body.

The total torque on the system about its center of mass equals the time derivative of the angular momentum in inertial coordinates:

$$
\boldsymbol{\tau}_{t}=\left\{\frac{d \mathbf{h}}{d t}\right\}_{N}
$$

Or, in a body-fixed frame, we get the classical Euler attitude equations:

$$
\boldsymbol{\tau}_{t}=\left\{\frac{d \mathbf{h}}{d t}\right\}_{b o d y}-\boldsymbol{\omega} \times(\mathbf{I} \boldsymbol{\omega})
$$

where $\left\{\frac{d \mathbf{h}}{d t}\right\}_{N}$ and $\left\{\frac{d \mathbf{h}}{d t}\right\}_{b o d y}$ are the angular momentum vector change of a rigid body with respect to the center of mass in an inertial frame and in a body frame, $\tau_{t}$ is the total external torque acting on the body, $\mathbf{I}$ is the matrix of moment of inertia of the rigid body and $\omega$ is the angular velocity vector in the body frame.

Note that these equations are found under the assumption that $\mathbf{I}$ is constant, i.e. a rigid body. We therefore imply that the deformations are slow with respect to any change in attitude, so changes in $\mathbf{I}$ can be neglected.

In this work, the modelled torques are: the solar radiation pressure torque $\boldsymbol{\tau}_{\text {rad }}$, the gravity gradient $\boldsymbol{\tau}_{G \text { rav }}$ and the magnetic torque $\tau_{M a g}$. The total torque is then:

$$
\boldsymbol{\tau}_{t}=\boldsymbol{\tau}_{r a d}+\boldsymbol{\tau}_{G \text { rav }}+\boldsymbol{\tau}_{M a g}
$$

\subsubsection{SRP torque}

From the solar radiation pressure force $\mathbf{F}_{\text {rad }}$ computed before, the torque is found as:

$$
\boldsymbol{\tau}_{r a d}=\boldsymbol{\rho}_{r a d} \times \mathbf{T} \mathbf{F}_{r a d}
$$


where $\boldsymbol{\rho}_{\text {rad }}$ is the vector from the center of mass of the object to the center of pressure, and $\mathbf{T}$ is the transformation matrix from the geocentric reference frame to the body reference system.

\subsubsection{Gravity gradient torque}

The gravitational torque for a rigid body is derived by determining the gravitational force $\mathrm{F}$ this equation can be written as [32]:

$$
\boldsymbol{\tau}_{G}=\frac{3 G M_{\oplus}}{r^{3}}[\mathbf{T} \hat{\mathbf{r}} \times \mathbf{I T} \hat{\mathbf{r}}]
$$

\subsubsection{Magnetic torque}

The motion of a conductor (i.e. a metallic object) in a magnetic field (i.e. that of the Earth) induces Eddy currents, creating forces and torques [31]. The induced torque can be given as:

$$
\boldsymbol{\tau}_{\text {Mag }}=k[\boldsymbol{\omega} \times \mathbf{T} \mathbf{B}] \times \mathbf{T} \mathbf{B}
$$

where $\mathbf{B}$ is the local Earth magnetic field, is approximated in polar coordinates as:

$$
\begin{aligned}
& B_{\theta}=\left(\frac{R_{\oplus}}{r}\right)^{3}\left[g_{1}^{0} \sin \theta-g_{1}^{1} \cos \theta \cos \phi-h_{1}^{1} \cos \theta \sin \phi\right] \\
& B_{\phi}=\left(\frac{R_{\oplus}}{r}\right)^{3}\left[g_{1}^{1} \sin \phi-h_{1}^{1} \cos \phi\right] \\
& B_{r}=2\left(\frac{R_{\oplus}}{r}\right)^{3}\left[g_{1}^{0} \cos \theta-g_{1}^{1} \sin \theta \cos \phi-h_{1}^{1} \sin \theta \sin \phi\right]
\end{aligned}
$$

where the coefficients for the Earth are: $g_{1}{ }^{0}=-30109 n T, g_{1}^{1}=-2006 n T, h_{1}^{1}=5704.2 n T$, and $\theta$ and $\phi$ are geodetic latitude and longitude respectively. Finally,

$$
k=\frac{\pi}{4} \sigma l^{3} A
$$

with $l$ the radius of the loop on the area of surface $A, \sigma$ the conductivity of the material.

\subsubsection{Moments of inertia}

In this research, as a preliminary assumption which we aim to remove in future work, and only for the purpose of easing the calculation of the matrix of inertia, we assume that the debris is flat. In principal axes of inertia, the matrix of inertia I becomes:

$$
\mathbf{I}=\left[\begin{array}{ccc}
I_{x x} & 0 & 0 \\
0 & I_{y y} & 0 \\
0 & 0 & I_{z z}
\end{array}\right]
$$

For a flat plate of dimensions $w, h$ and $d$ : 


$$
\begin{aligned}
& I_{x x}=\frac{1}{12} m\left(h^{2}+d^{2}\right) \\
& I_{y y}=\frac{1}{12} m\left(w^{2}+d^{2}\right) \\
& I_{z z}=\frac{1}{12} m\left(w^{2}+h^{2}\right)
\end{aligned}
$$

\subsection{Rotational kinematics}

The attitude of a tumbling body is most conveniently integrated with a set of axes fixed to the body (principal axes of inertia). It is therefore required to parameterize the attitude to obtain the transformation matrix $\mathbf{R}$ from the body axes (superscript B) to a set of inertial coordinates $(\mathrm{N})$ :

$$
\mathbf{v}^{B}=\left[\begin{array}{lll}
R_{11} & R_{12} & R_{13} \\
R_{21} & R_{22} & R_{23} \\
R_{31} & R_{32} & R_{33}
\end{array} \mathbf{v}^{N}\right.
$$

The rotation matrix $\mathbf{R}$ can be expressed as a function of pitch $(\varphi)$, yaw $(\psi)$ and roll $(\theta)$ angles, by using a 3-1-3 Euler rotation, as [32].

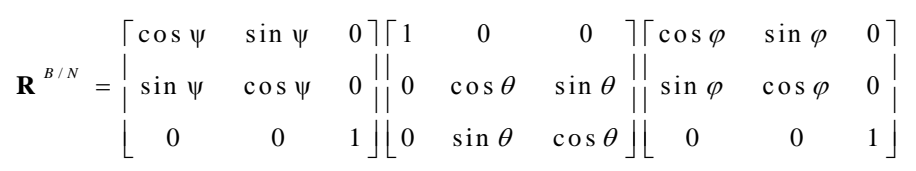

leading to:

$$
\mathbf{R}^{B / N}=\left[\begin{array}{ccc}
\mathrm{c}(\psi) c(\varphi)-s(\psi) \mathrm{c}(\theta) s(\varphi) & \mathrm{c}(\psi) \mathrm{c}(\theta) s(\varphi)+s(\psi) c(\varphi) & s(\theta) s(\psi) \\
-\mathrm{c}(\psi) s(\varphi)-s(\psi) \mathrm{c}(\theta) s(\varphi) & \mathrm{c}(\psi) \mathrm{c}(\theta) c(\varphi)-s(\psi) s(\varphi) & s(\theta) s(\varphi) \\
s(\psi) \mathrm{s}(\theta) & -c(\psi) \mathrm{s}(\theta) & c(\theta)
\end{array}\right]
$$

where $\cos ()$ is abbreviated by "c()" and $\sin ()$ by "s () ".

While Euler angles provide an intuitive way of defining the attitude at any given time, they have singularities and therefore they are not suitable for being used in automatic integration.

\subsubsection{Quaternions}

Quaternions are an alternative way of parameterizing the attitude, with no singularities, at the cost of using four scalar variables instead of three. It is possible to rotate from an initial orientation to final orientation with a single rigid rotation, around an eigenvector $\mathbf{e}$, defining a principal rotation axis, of a principal rotation angle $\Phi$, through the matrix:

$$
\begin{aligned}
\mathbf{R}=\cos (\Phi) 1+[1-\cos (\Phi)] & {\left[\begin{array}{lll}
e_{1} & e_{2} & e_{3}
\end{array}\right]\left[\begin{array}{lll}
e_{1} & e_{2} & e_{3}
\end{array}\right]^{T}-\sin (\Phi) \mathbf{E} } \\
\text { with: } \mathbf{E}= & {\left[\begin{array}{ccc}
0 & -e_{3} & e_{2} \\
e_{3} & 0 & -e_{1} \\
-e_{2} & e_{1} & 0
\end{array}\right] }
\end{aligned}
$$


or:

$$
\mathbf{R}=\left[\begin{array}{ccc}
\cos (\Phi)+e_{1}^{2}(1-\cos (\Phi)) & e_{1} e_{2}(1-\cos (\Phi))+e_{3} \sin (\Phi) & e_{1} e_{3}(1-\cos (\Phi))-e_{2} \sin (\Phi) \\
e_{1} e_{2}(1-\cos (\Phi))-e_{3} \sin (\Phi) & \cos (\Phi)+e_{2}^{2}(1-\cos (\Phi)) & e_{2} e_{3}(1-\cos (\Phi))+e_{1} \sin (\Phi) \\
e_{1} e_{3}(1-\cos (\Phi))+e_{2} \sin (\Phi) & e_{2} e_{3}(1-\cos (\Phi))-e_{1} \sin (\Phi) & \cos (\Phi)+e_{3}^{2}(1-\cos (\Phi))
\end{array}\right]
$$

The principal axis can be found starting from $\mathbf{R}$ as:

$$
\begin{aligned}
& e_{1}=\frac{\left(R_{23}-R_{32}\right)}{2 \sin \Phi} \\
& e_{2}=\frac{\left(R_{31}-R_{13}\right)}{2 \sin \Phi} \\
& e_{3}=\frac{\left(R_{12}-R_{21}\right)}{2 \sin \Phi}
\end{aligned}
$$

Finally, the quaternions can be defined as:

$$
\begin{aligned}
& q_{1}=e_{1} \sin \left(\frac{\Phi}{2}\right) \\
& q_{2}=e_{2} \sin \left(\frac{\Phi}{2}\right) \\
& q_{3}=e_{3} \sin \left(\frac{\Phi}{2}\right) \\
& q_{4}=\cos \left(\frac{\alpha}{2}\right)
\end{aligned}
$$

or, in vector notation: $\mathbf{q}=\left[\begin{array}{l}q_{1} \\ q_{2}\end{array} \mid\right.$

The singularity is removed because only three of the quaternions are actually necessary to reconstruct the attitude, and the following relationship, instead of one of the above, can be used to find the value of the singular quaternion:

$$
q_{1}^{2}+q_{2}^{2}+q_{3}^{2}+q_{4}^{2}=1
$$

Finally, the cosine matrix to transform an arbitrary vector can be expressed directly as a function of the quaternions as:

$$
\mathbf{R}=\left[\begin{array}{lll}
1-2\left(q_{2}^{2}+q_{3}^{2}\right) & 2\left(q_{1} q_{2}+q_{3} q_{4}\right) & 2\left(q_{1} q_{3}-q_{2} q_{4}\right) \\
2\left(q_{1} q_{2}-q_{3} q_{4}\right) & 1-2\left(q_{1}^{2}+q_{3}^{2}\right) & 2\left(q_{2} q_{3}-q_{1} q_{4}\right) \\
2\left(q_{1} q_{3}+q_{2} q_{4}\right) & 2\left(q_{2} q_{3}-q_{1} q_{4}\right) & 1-2\left(q_{1}^{2}+q_{2}^{2}\right)
\end{array}\right]
$$

and vice-versa: 


$$
\begin{aligned}
& q_{1}=\frac{\left(R_{23}-R_{32}\right)}{4 q_{4}} \\
& q_{2}=\frac{\left(R_{31}-R_{13}\right)}{4 q_{4}} \\
& q_{3}=\frac{\left(R_{12}-R_{21}\right)}{4 q_{4}} \\
& q_{4}=\sqrt{\frac{R_{11}+R_{22}+R_{33}+1}{2}}
\end{aligned}
$$

Similar equations exist in case $q_{4}=0$, which make use of any of the other three quaternions at the denominator.

Given the initial attitude of the spacecraft, expressed as a quaternion, the attitude at subsequent times can be tracked, knowing the angular velocity in body axes from the Euler equations, by integrating the following differential equations of motion for the quaternions:

$$
\begin{aligned}
& \frac{d \mathbf{q}}{d t}=\frac{1}{2} \boldsymbol{\Omega} \mathbf{q} \\
\text { with: } \boldsymbol{\Omega}= & {\left[\begin{array}{cccc}
0 & \omega_{3} & -\omega_{2} & \omega_{1} \\
-\omega_{3} & 0 & 0 & \omega_{2} \\
\omega_{2} & -\omega_{1} & -\omega_{3} & 0 \\
-\omega_{1} & -\omega_{2} & -\omega_{3} & 0
\end{array}\right] }
\end{aligned}
$$

\subsection{Validation}

The diagram in Fig. 11 shows how the integration of orbital and attitude dynamics is preformed, with the interdependency of each one of the torques and forces. The propagation is initiated with the six initial Keplerian elements to define the initial position and velocity, and Euler angles (roll, pitch and yaw) and angular rate for the attitude. The integrator is the Runge-Kutta solver ODE45 provided in MATLAB. The following sections show some of the tests that were done for validating the algorithm and its inner functions. 


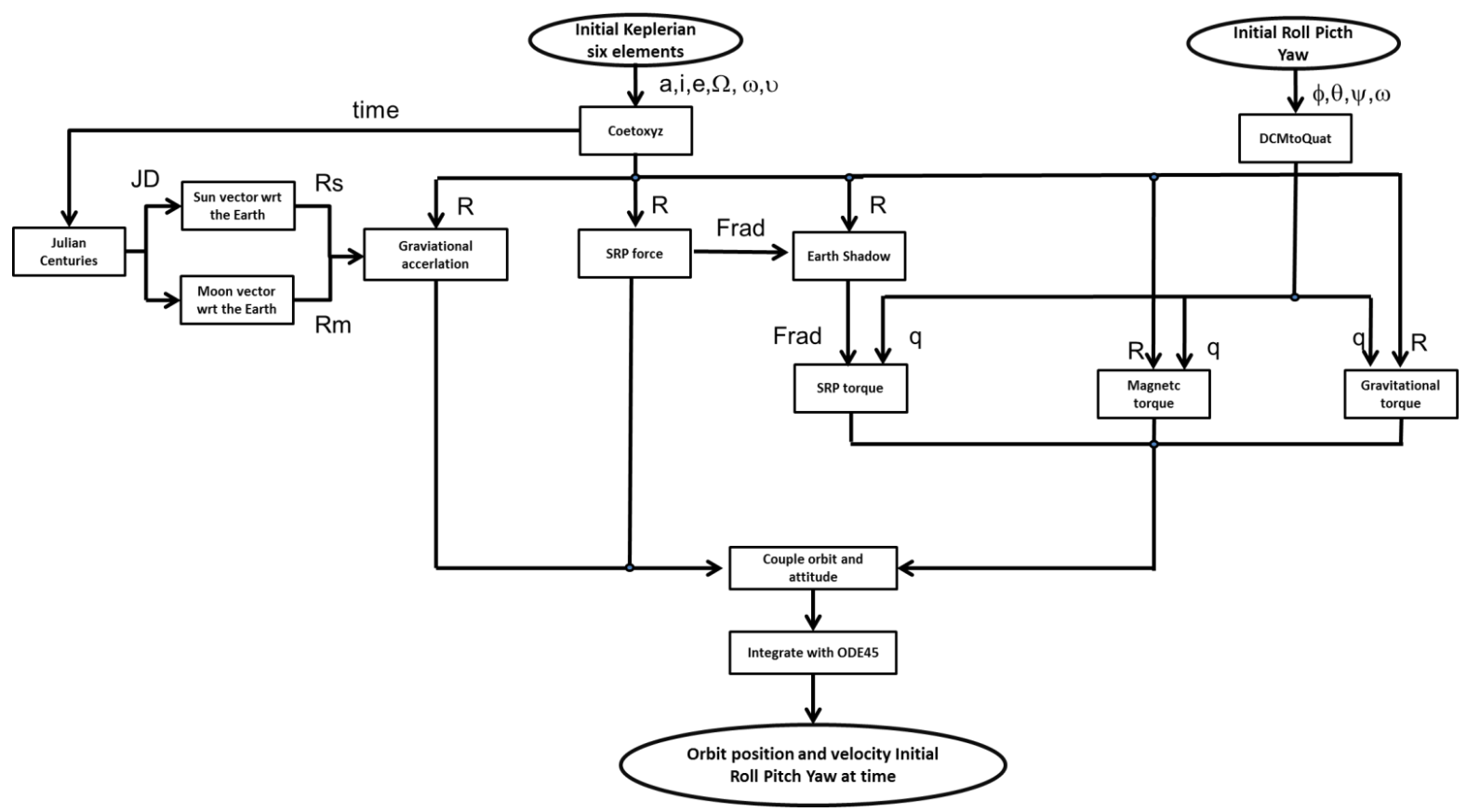

Fig. 11. Propagation of orbital and attitude dynamics.

\subsubsection{Solar radiation pressure force}

To validate the solar radiation pressure force, we consider a thin flat plate, with same reflectivity properties on both front and back sides. The the position vector of the object and the Sun with respect to Earth are fixed, while the incidence angle is varied over the full circle (Fig. 12).

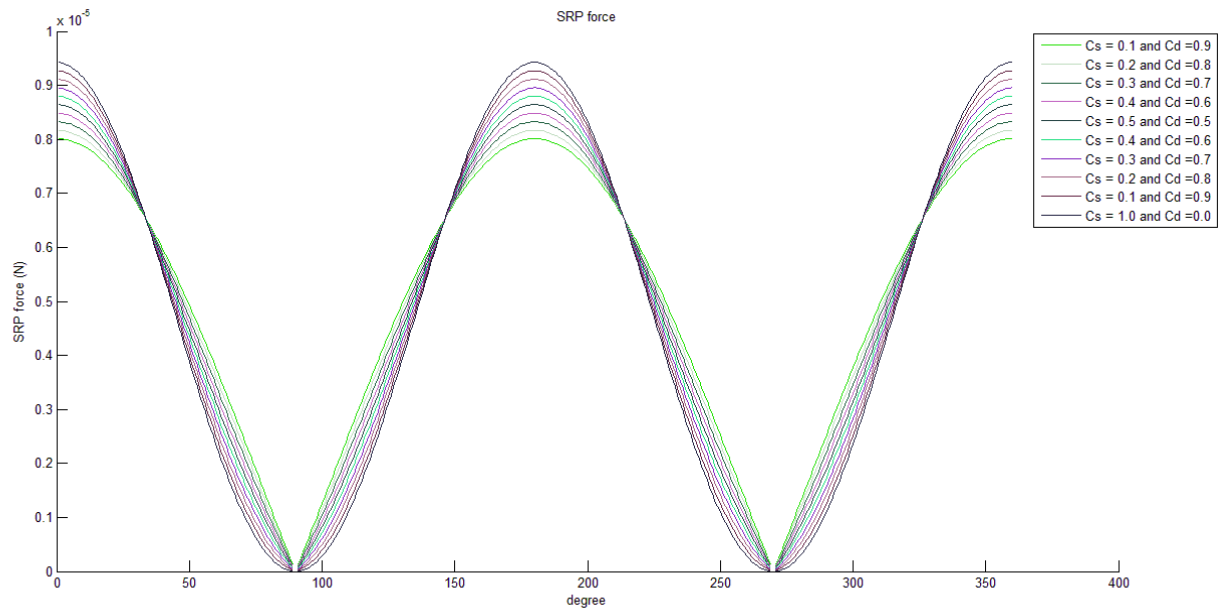

Fig. 12. Solar radiation pressure force result by varying incident angle

The front side of the plate is subject to SRP between $0^{\circ}-90^{\circ}$ and $270^{\circ}-360^{\circ}$, while in the remaining interval between $90^{\circ}-270^{\circ}$ the back side is illuminated. Due to the cosine law, the maximum magnitude of the force is at $0^{\circ}$ and $180^{\circ}$ (plate perpendicular to the sun direction) and null at $90^{\circ}$ and $270^{\circ}$ (plate tangent to sun direction). 


\subsubsection{Shadow effect}

The object is placed in several positions with respect to the Sun and the Earth, and it is checked that the returned value for the SRP is either the one in lighting conditions or zero when inside the Earth's umbra.

\subsubsection{Orbital dynamics}

We consider a flat plate whose characteristics are in Table 1. The dynamics of the orbit is propagated starting from initial Keplerian elements in Table 2, for 10 orbits. Orbit of the objects is simulated by equation (3.9) and set the initial equation in Table 2. Gravitational perturbation of the Sun, Earth and Moon are considered. The resulting orbit in the physical space is plotted in Fig. 13, and the evolution of the 5 slowly-changing Keplerian elements over time is plotted in Fig. 14.

Table 1. Object data

\begin{tabular}{ll}
\hline Object characteristic & Value \\
\hline Mass $(\mathrm{kg})$ & 10 \\
Area $\left(\mathrm{m}^{2}\right)$ & 1 \\
Angular velocity $[\mathrm{x}, \mathrm{y}, \mathrm{z}](\mathrm{rad} / \mathrm{s})$ & {$[0,0,0]$} \\
Specular reflection coefficient & 0.6 \\
Diffuse reflection coefficient & 0.26 \\
Absorption coefficient & 0.14 \\
Conduction $(0.25 \mathrm{~mm})$ & $36.59 \times 10^{6}$ \\
\hline
\end{tabular}

Table 2. Initial conditions

\begin{tabular}{ll}
\hline Keplerian element & Value \\
\hline Semi-major axis (km) & 42,164 \\
Mean anomaly (degrees) & $270^{\circ}$ \\
Argument of perigee (degrees) & $90^{\circ}$ \\
Ascending node (degrees) & $60^{\circ}$ \\
Eccentricity & 0.0001 \\
Inclination (degrees) & $40^{\circ}$ \\
\hline
\end{tabular}


Fig. 13. Trajectory.

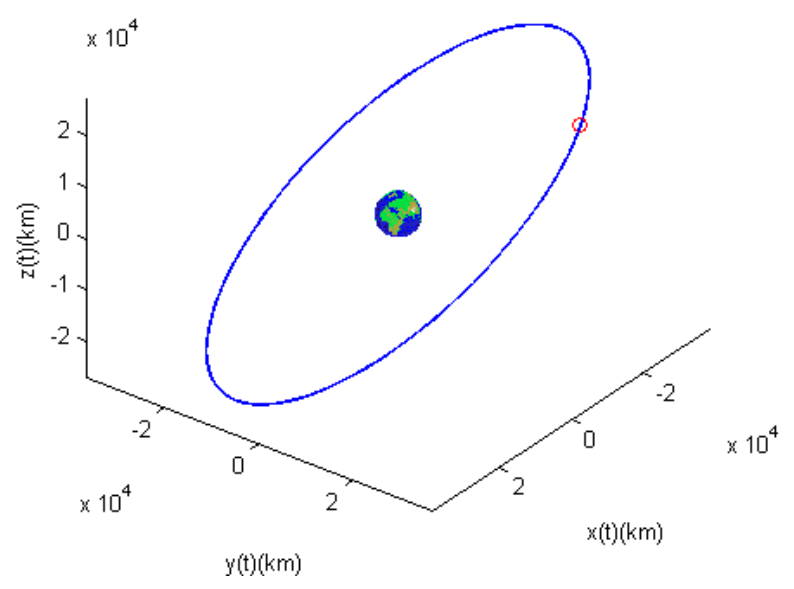



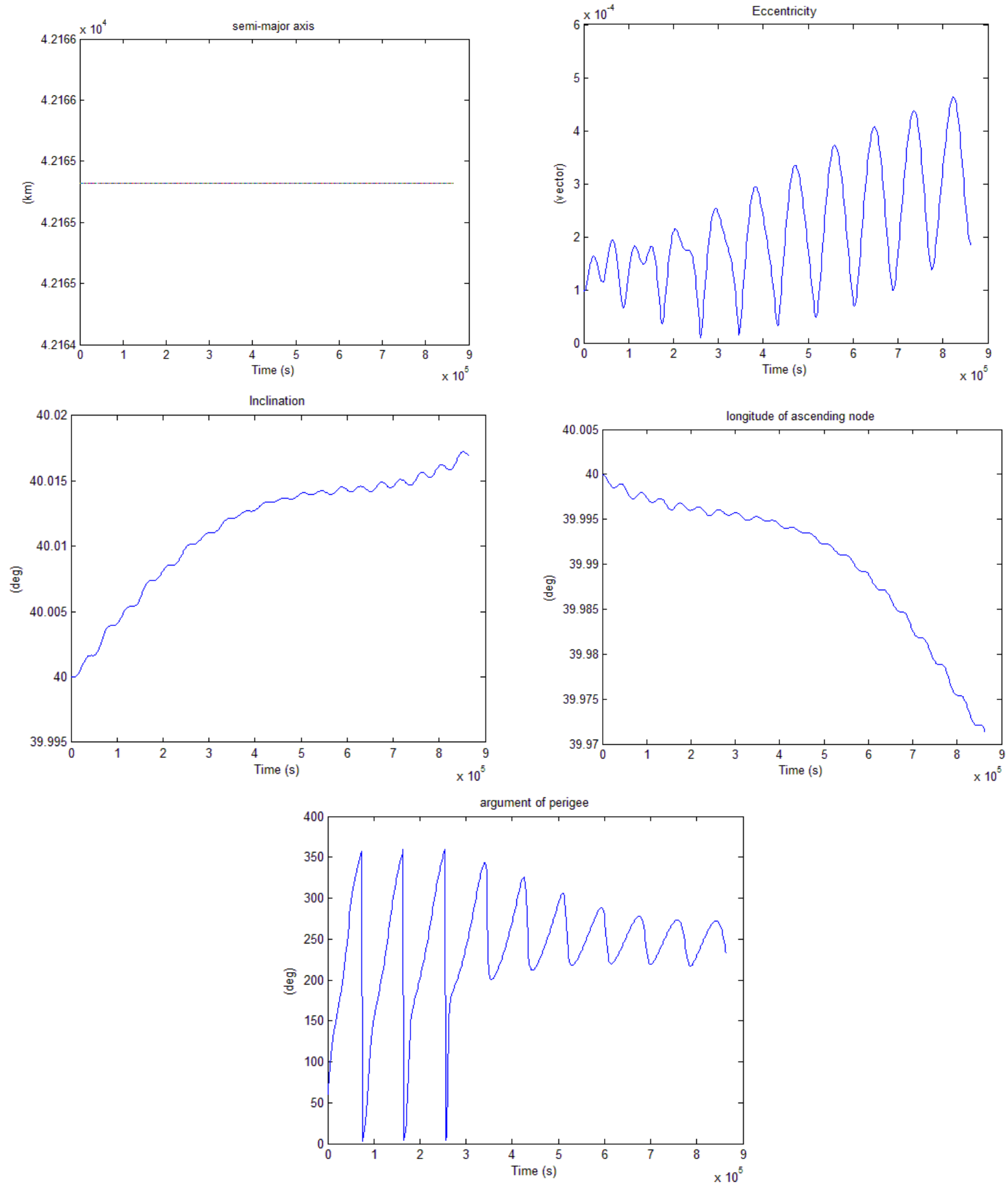

Fig. 14. Keplerian elements over time.

\subsubsection{Torque-free attitude dynamics}

The attitude dynamics is validated by setting no external torques and propagating in time. The moments of inertia are arbitrarily set as $I_{x}=1.0, I_{y}=0.8$ and $I_{z}=0.5 \mathrm{~kg} \cdot \mathrm{m}^{2}$ and the initial angular velocity to $\omega_{0}=[5,1,3] \mathrm{rad} / \mathrm{s}$. In torque-free motion, the angular momentum vector follows a periodic trajectory as expected, plotted in Fig. 15. In 
addition, the angular velocity vector follow the trajectory given by the intersection of the ellipsoid of inertia and the ellipsoid of angular momentum, which are plotted in Fig. 16, matching to the results in [32].

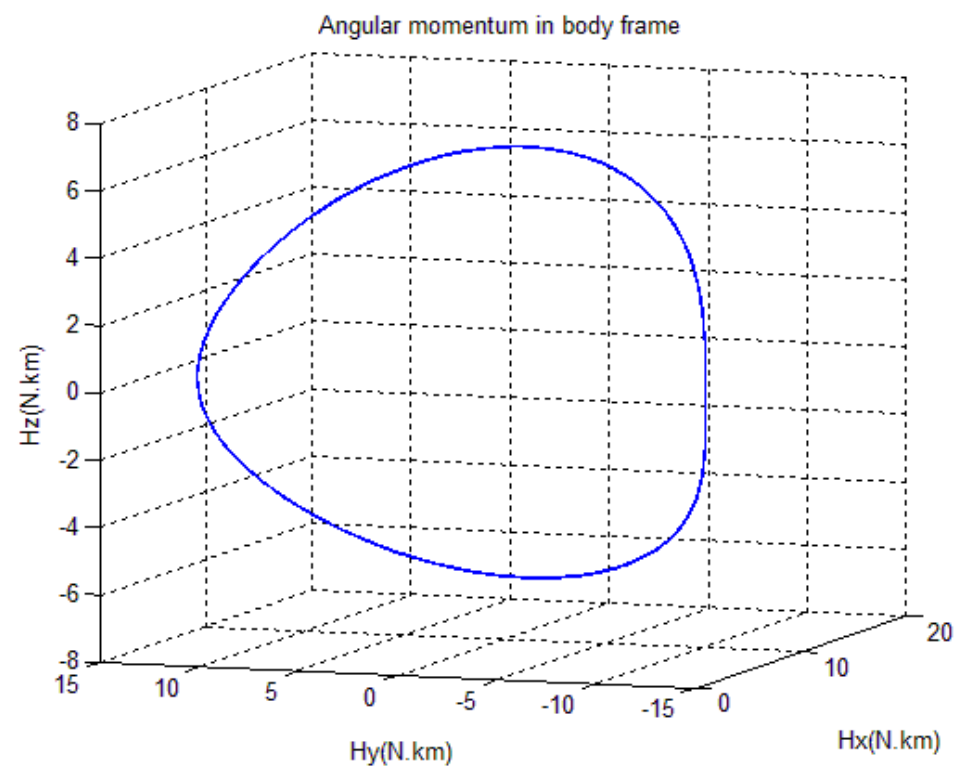

Fig. 15. Trajectory of angular momentum in body frame.

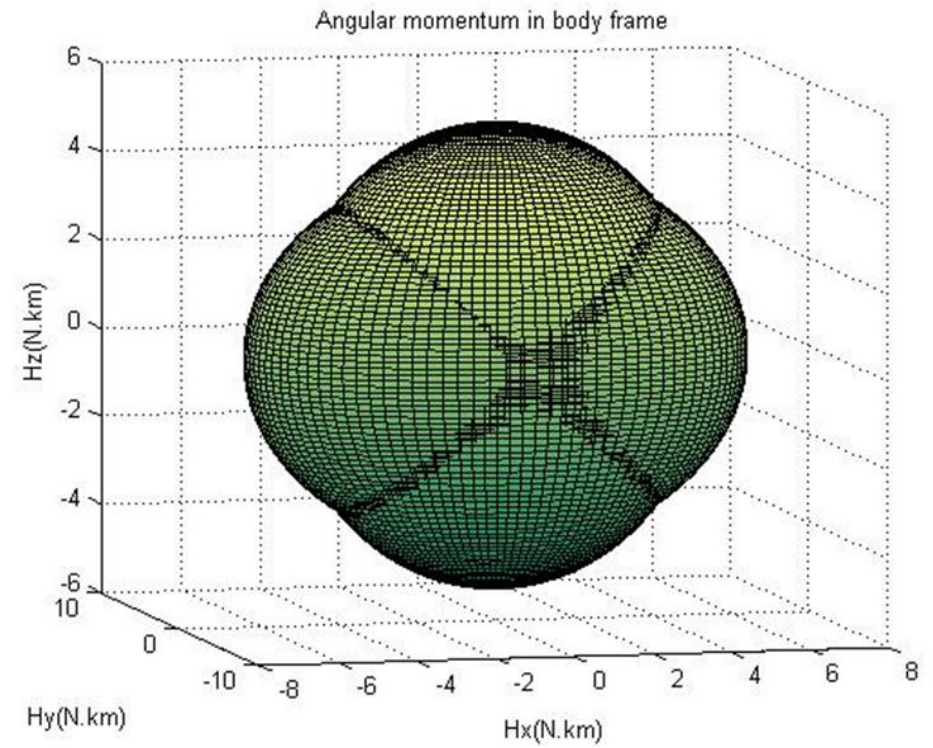

Fig. 16. Ellipsoid of energy and ellipsoid of angular momentum.

\subsubsection{Coupled orbit and attitude dynamics}

The propagation of coupled attitude and orbital dynamics is presented here, for 10 days (Fig. 17, Fig. 18 and Fig. 19), 100 days (Fig. 20, Fig. 21 and Fig. 22), and 1 year or 365 days (Fig. 23, Fig. 24 and Fig. 25). Inclination, eccentricity and longitude of ascending node for 365 days change only slightly. The longitude of ascending node 
decreases as inclination increases up about $0.5^{\circ}$. The eccentricity initially increases to up to 0.001 and then it goes back to zero after the full year. This behavior is expected and it is due to the (apparent) rotation of the sun around the Earth. 365 days. The plots of roll, pitch and yaw Euler angles show tumbling on all axes.

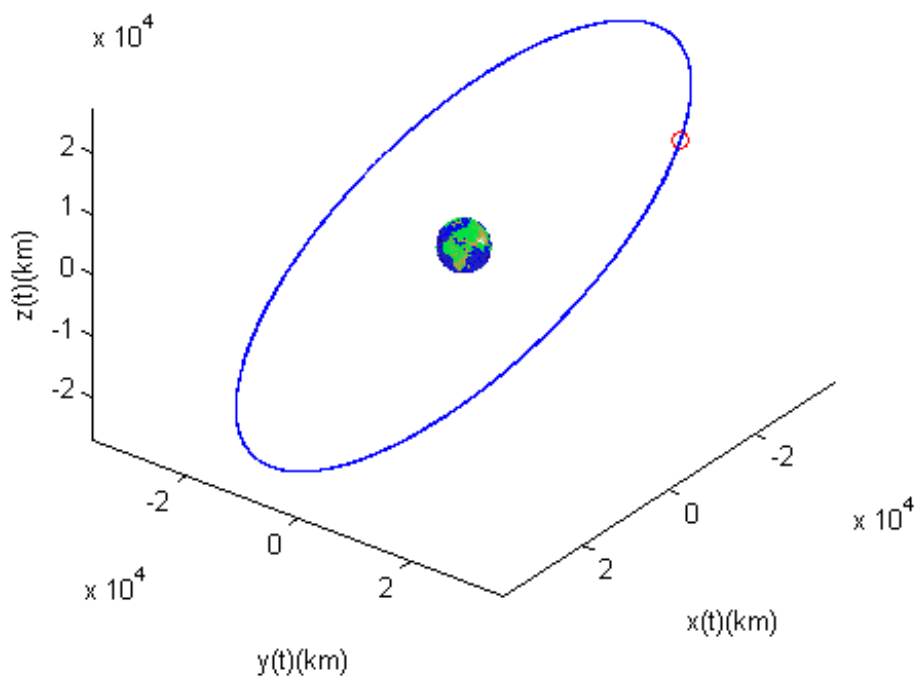

Fig. 17. 10 days, orbit. 

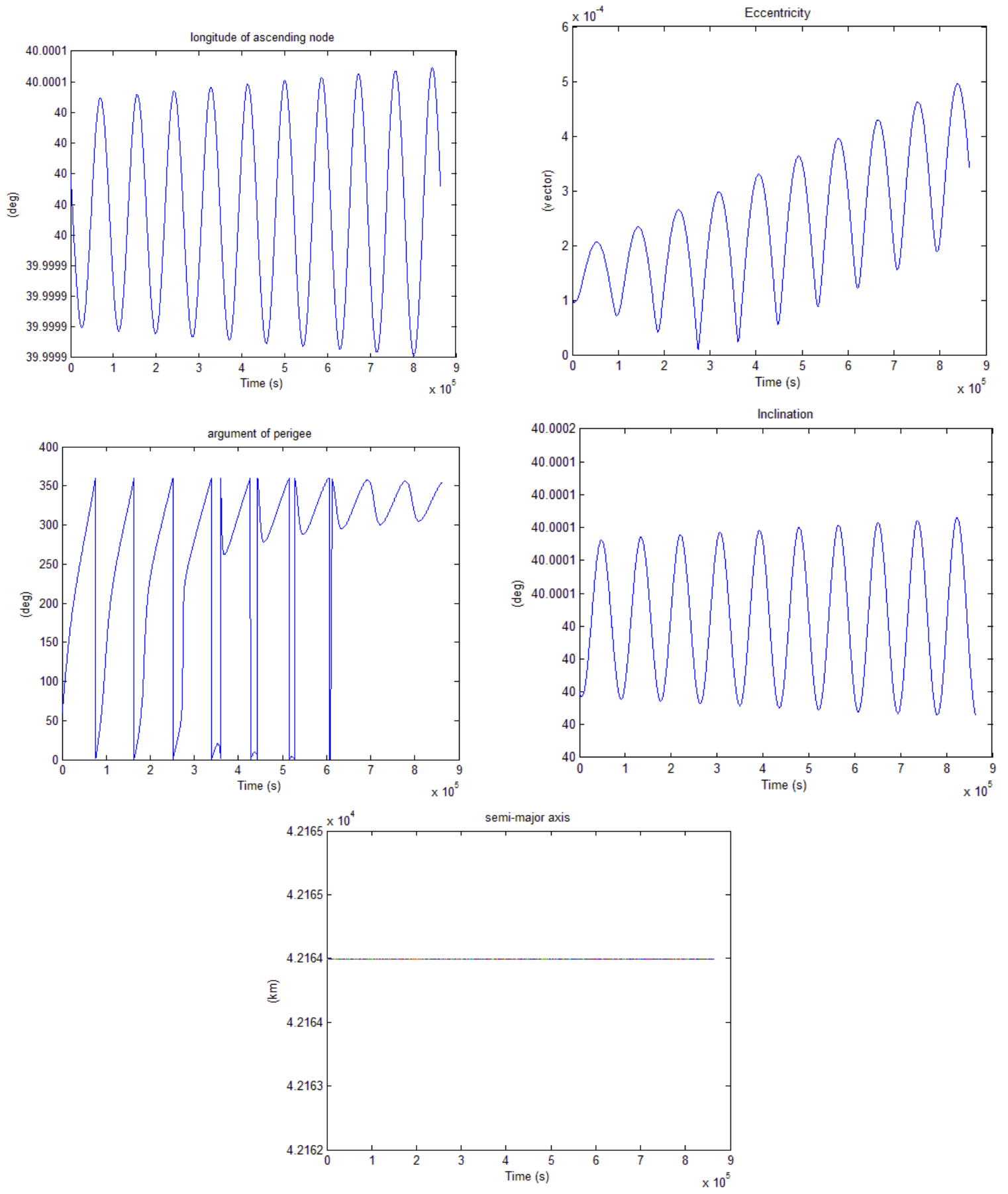

Fig. 18. 10 days, Keplerian elements. 

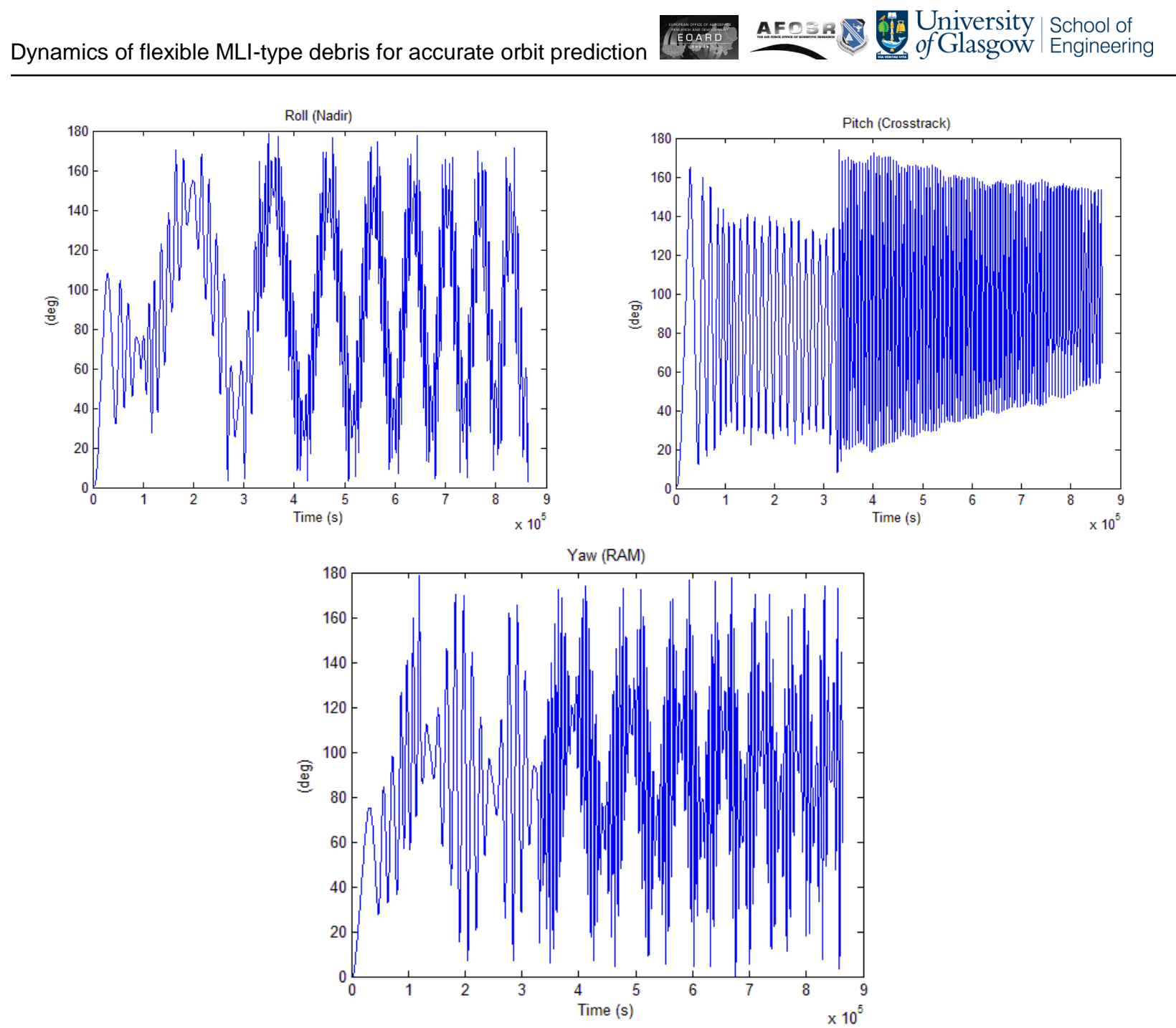

Fig. 19. 10 days, Euler angles. 


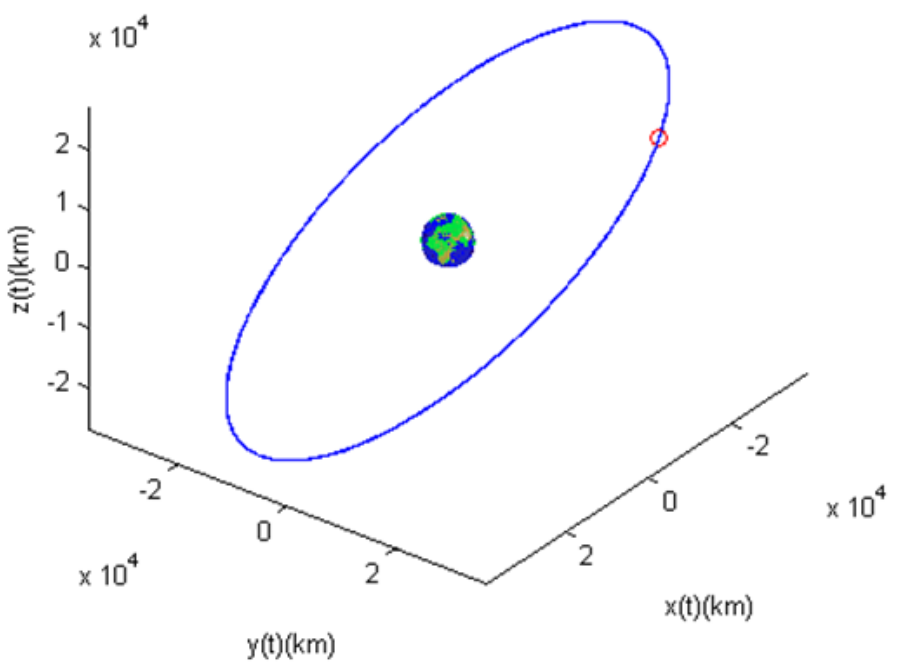

Fig. 20. 100 days, orbit. 

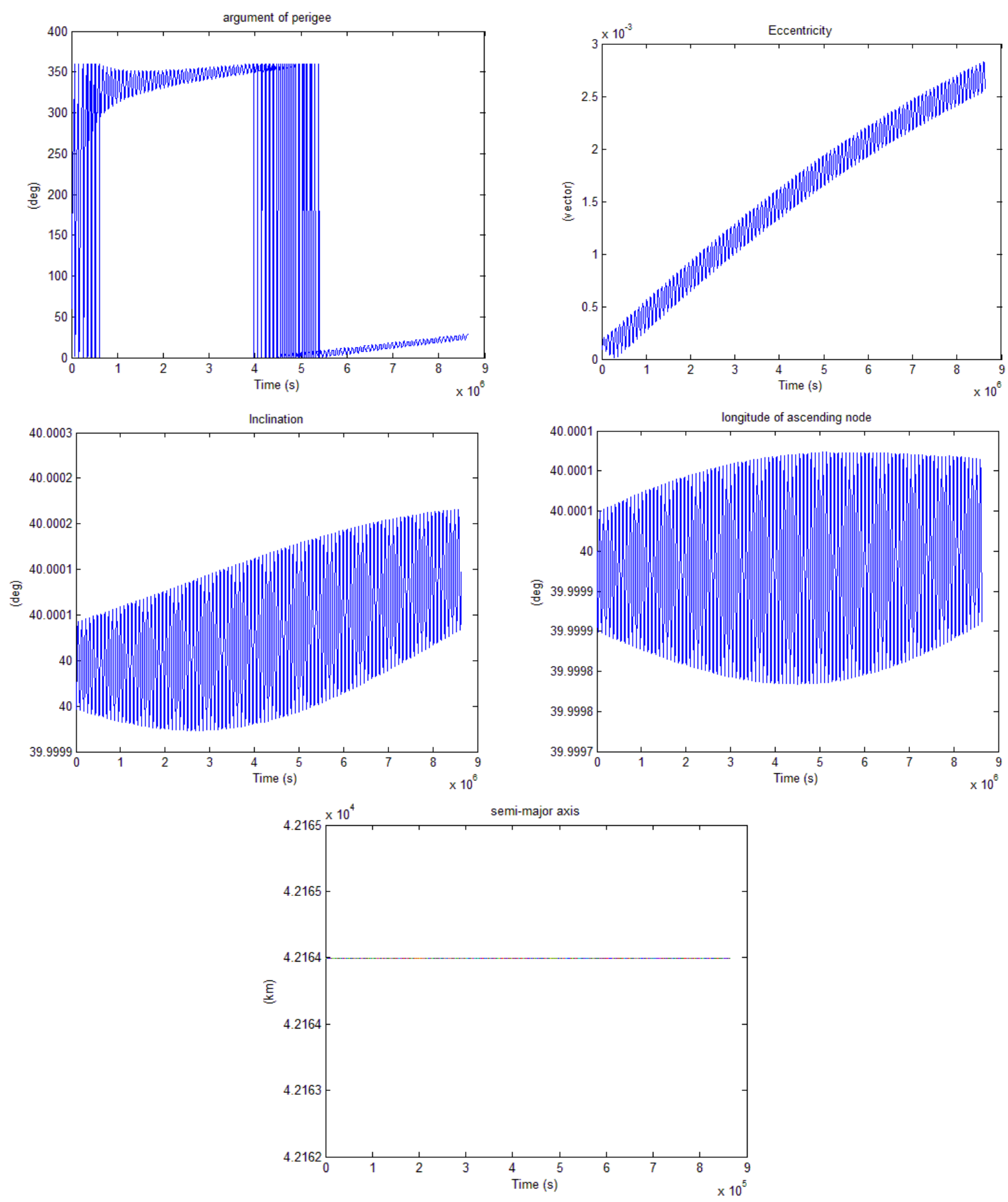

Fig. 21. 100 days, Keplerian elements. 

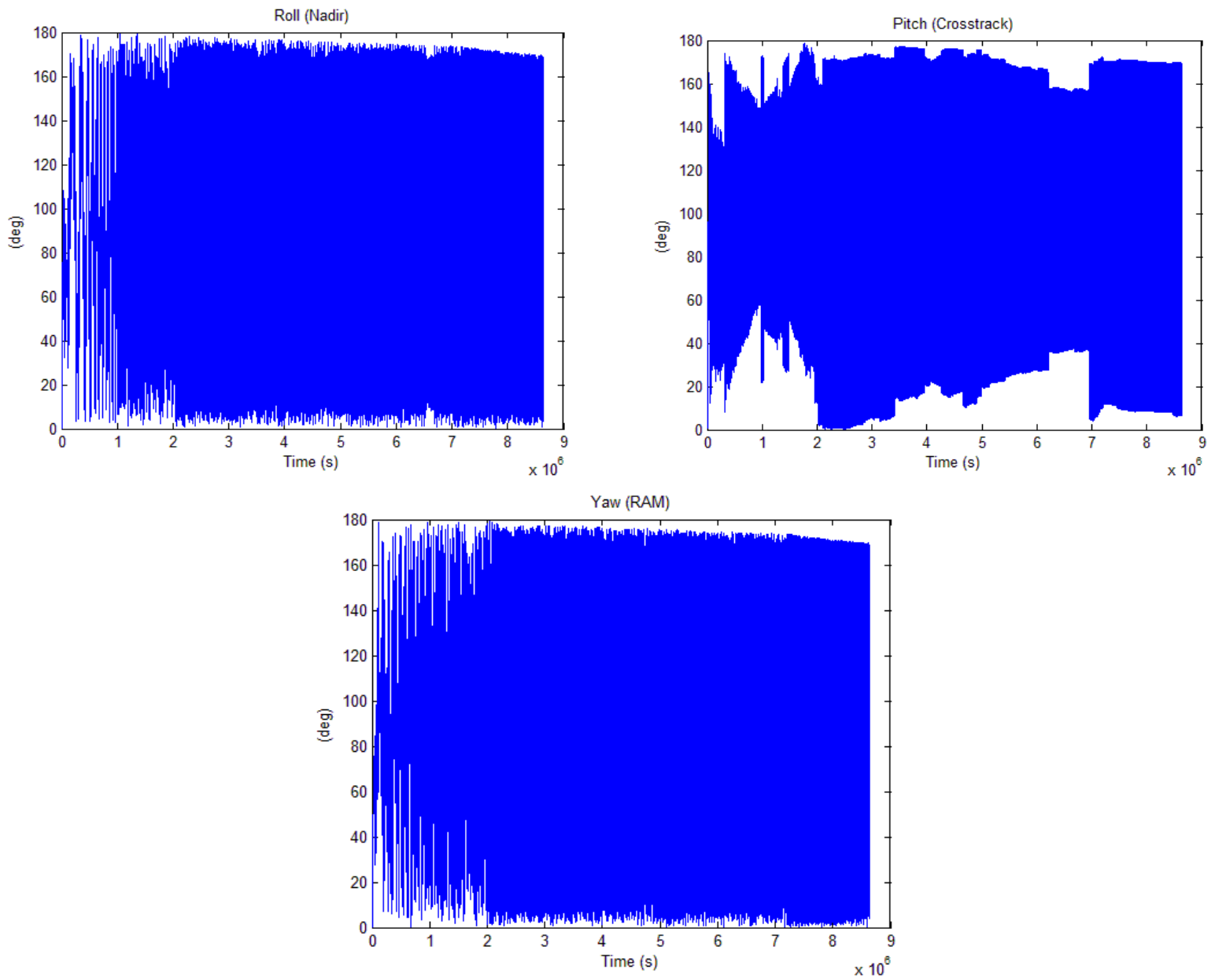

Fig. 22. 100 days, Euler angles

Debris Orbit

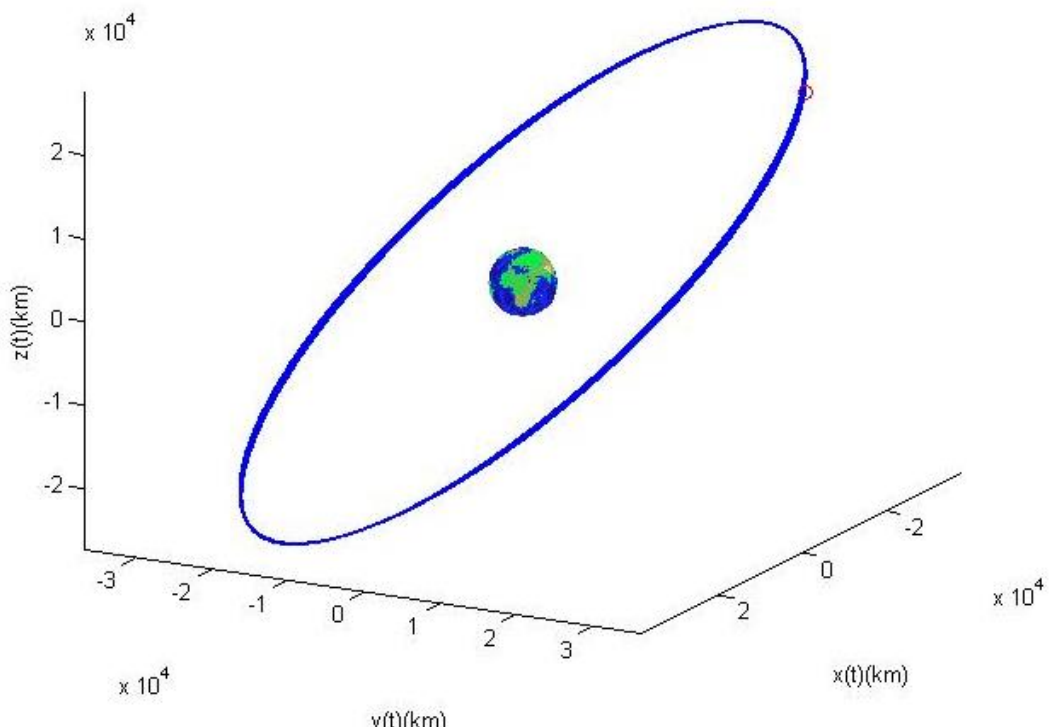

Fig. 23. 365 days, orbit. 

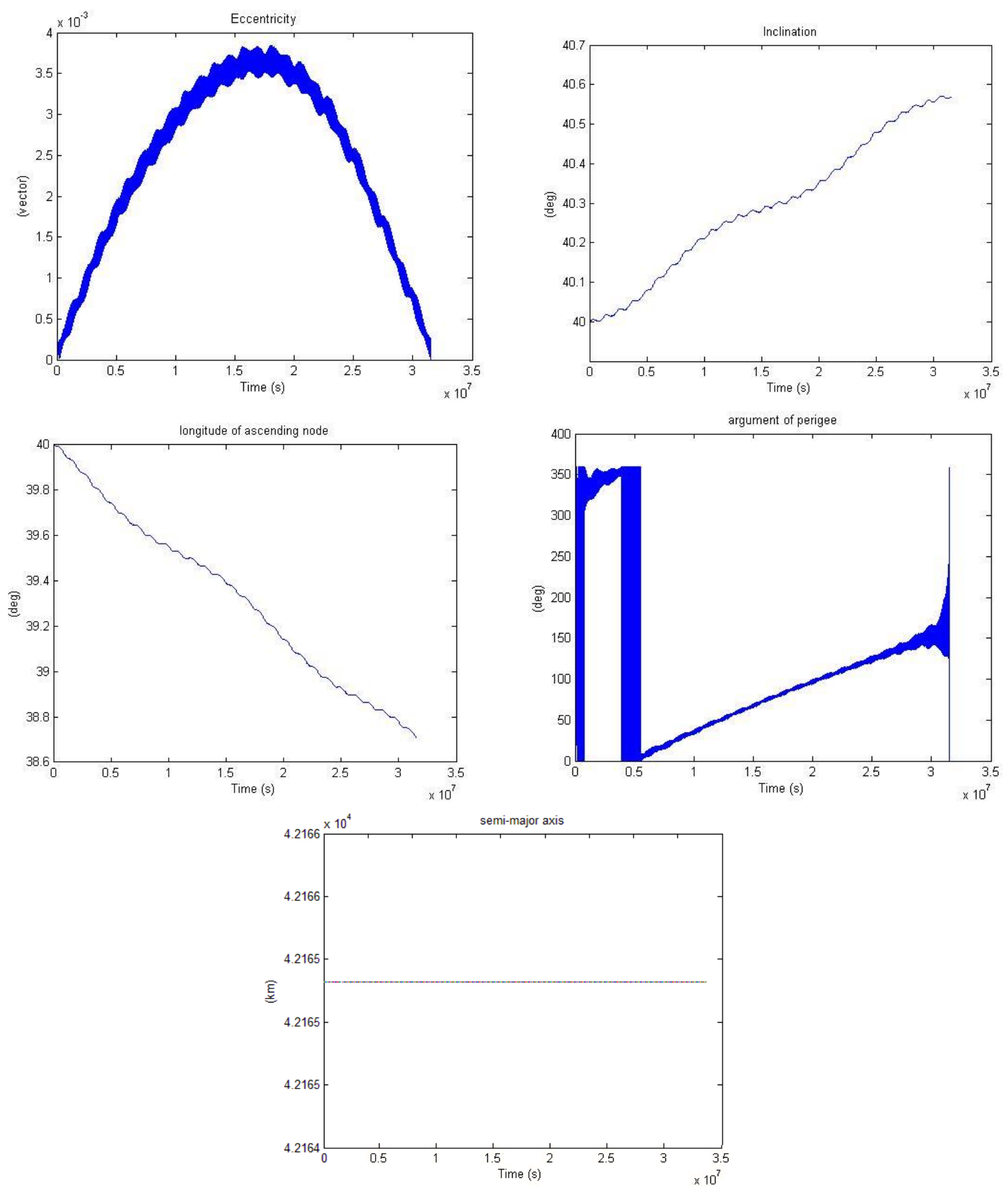

Fig. 24. 365 days, Keplerian elements. 

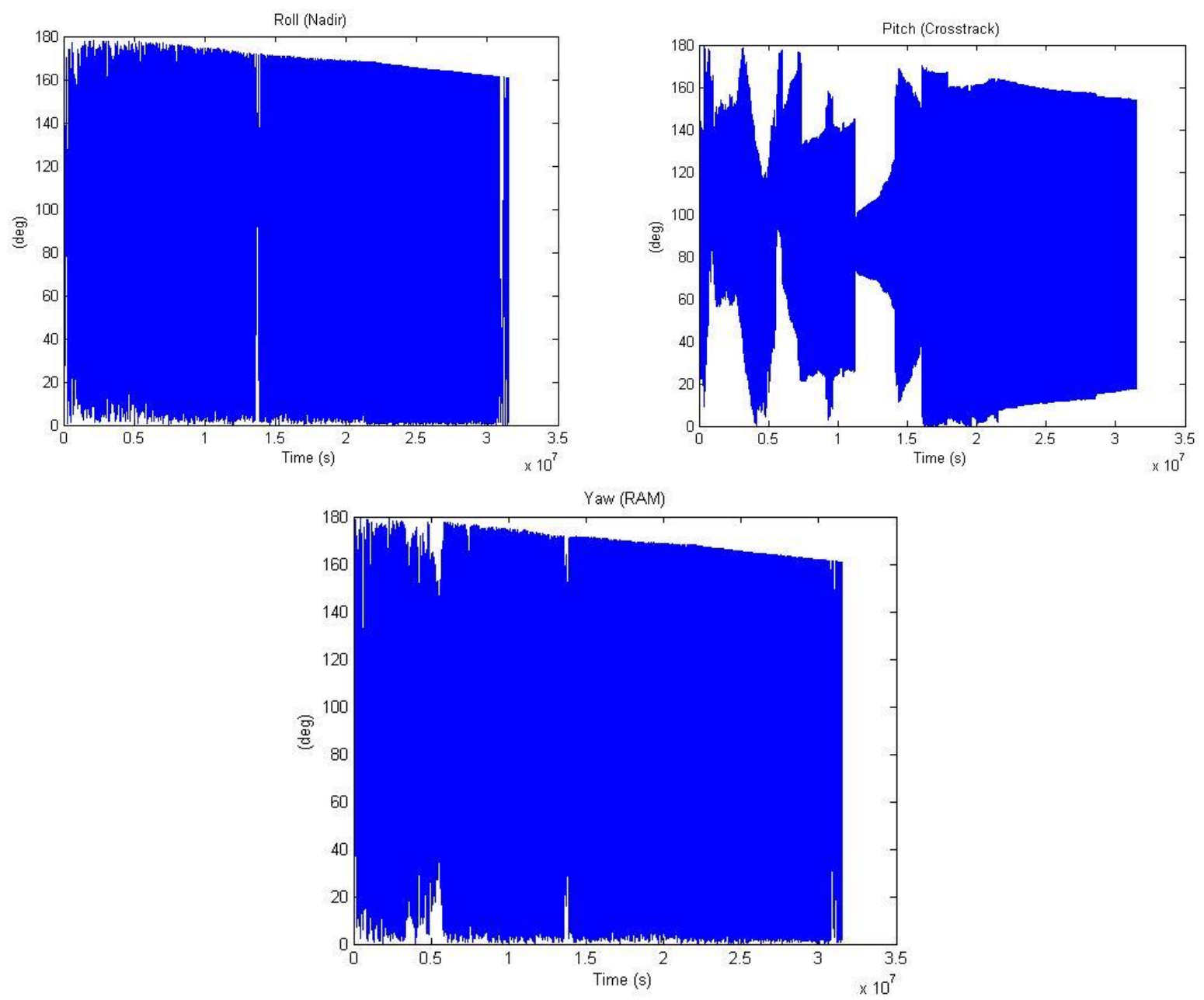

Fig. 25. 365 days, Euler angles. 


\section{Orbital and attitude dynamics of flexible debris - Bernoulli-Euler model}

The results show that the different initial conditions of attitude motion results in significant changes in term of orbit element and the shape of the MLI debris influences unique attitude motion and the volume entity of the disturbances. Thus, it is believed that the deformation is one of the main contributing factors to increase the accuracy of orbit determination of flexible debris.

In this paper, we will use a Finite Element Method (FEM) to model the deformation of flexible debris considering only in one plane for this preliminary study and propagate the coupling of orbit and attitude dynamics over orbits in the GEO region in 3D. We will consider two configurations: flat sheet and folded flat plate and try to understand their evolution subject to environmental perturbations due to Earth gravitation, third body gravitation from the Sun and Moon and the solar radiation pressure.

The work described in this chapter was presented at the 64th International Astronautical Congress, Beijing, China [33].

\subsection{Finite element analysis}

The Bernoulli-Euler theory [34] is used here to investigate the deformation of debris. Fig. 26 represents a beam with two nodes. $E$ is Young's Modulus, $A$ is cross-section and $I$ is the second moment of inertia and each node has three global degrees of freedom, two displacement in $\mathrm{x}$, $\mathrm{y}$ axis and one rotation.

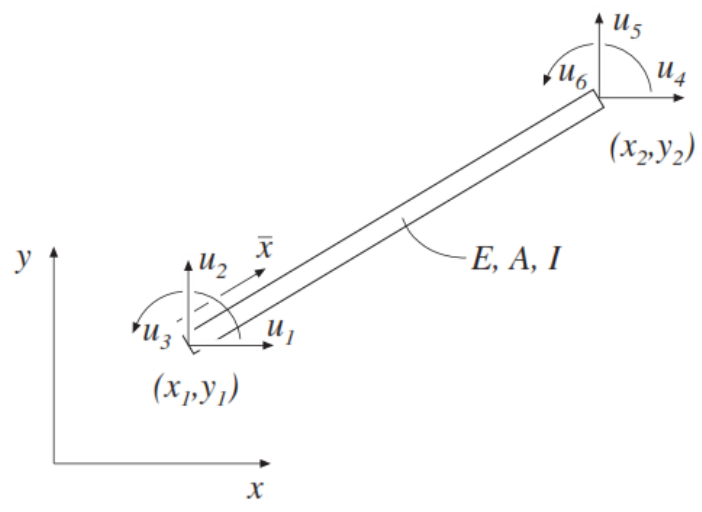

Fig. 26. Bernoulli beam element with 2 nodes

The vector of displacement is given by:

$$
U=\left[\begin{array}{llllll}
U_{1} & U_{2} & U_{3} & U_{4} & U_{5} & U_{6}
\end{array}\right]^{T}
$$


where $U_{1}, U_{2}$ and $U_{3}$ are $\mathrm{x}$ and $\mathrm{y}$ displacement and rotation of the $1^{\text {st }}$ node respectively, $U_{4}, U_{5}$ and $U_{6}$ are $\mathrm{x}$ and $\mathrm{y}$ displacement and rotation of the $2^{\text {nd }}$ node respectively. In this study, the debris will have nine degrees of freedom space. To simplify only the linear dynamics will be considered:

$$
M \ddot{U}+C \dot{U}+K U=F
$$

where $U, \dot{U}$ and $\ddot{U}$ are the vectors of generalized displacement, velocity and acceleration respectively :

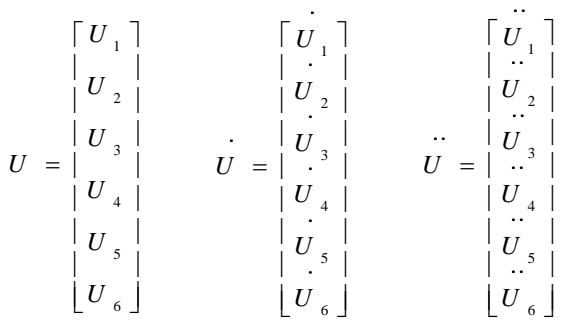

Also, $F$ is the force matrix which consists of forces along the $\mathrm{x}$ and $\mathrm{y}$ axis and $M$ :

$$
F=\left[\begin{array}{ll}
F_{x 1} & \vdots \\
F_{y 1} & \mid \\
M_{1} & \mid \\
F_{x 2} & \mid \\
F_{y 2} \\
M_{2}
\end{array}\right]
$$

$M$ is the mass matrix standard of Bernoulli-Euler Beam element in local coordinates:

$$
\bar{M}=\frac{m L}{420}\left[\begin{array}{cccccc}
140 & 0 & 0 & 70 & 0 & 0 \\
0 & 156 & 22 L & 0 & 54 & -13 L \\
0 & 22 L & 4 L^{2} & 0 & 13 L & -3 L^{2} \\
70 & 0 & 0 & 140 & 0 & 0 \\
0 & 54 & 13 L & 0 & 156 & -22 L \\
0 & -13 L & -3 L^{2} & 0 & -22 L & 4 L^{2}
\end{array}\right]
$$

\subsection{Coordinate transformation}

We can transform from a body frame of reference to an inertial frame of reference by using a transformation matrix:

$$
T=\left[\begin{array}{cccccc}
c & s & 0 & 0 & 0 & 0 \\
-s & c & 0 & 0 & 0 & 0 \\
0 & 0 & 1 & 0 & 0 & 0 \\
0 & 0 & 0 & c & s & 0 \\
0 & 0 & 0 & -s & c & 0 \\
0 & 0 & 0 & 0 & 0 & 1
\end{array}\right]
$$

where 


$$
c=\cos \theta=\frac{x_{2}-x_{1}}{L} \text { and } s=\sin \theta=\frac{y_{2}-y_{1}}{L}
$$

Thus, the stiffness matrix and mass matrix in inertial coordinates are defined as:

$$
K=T^{T} \bar{K} T \text { and } M=T^{T} \bar{M} T
$$

Finally, the most common approach is to define the damping matrix $C$, through Rayleigh damping [35], which assumes a proportionality to the mass $\bar{M}$ and stiffness $\bar{K}$. This equation is given by:

$$
C=\alpha \bar{M}+\beta \bar{K}
$$

where $\alpha$ and $\beta$ are proportional damping coefficients

\subsection{Simulation analysis}

The aim of this analysis is to compare the orbit propagation of a debris considered as rigid body and the propagation of a debris including its deformation. According to most observations, MLI debris is found in the GEO region. We will assume the initial orbital parameters are defined as in Table 3.

Table 3 The initial six Keplerian elements

\begin{tabular}{ll}
\hline \multicolumn{1}{c}{ Keplerian elements } \\
\hline$a(\mathrm{~km})$ & 41,254 \\
$e$ & 0.0001 \\
$i(\mathrm{deg})$ & 30 \\
$\Omega(\mathrm{deg})$ & 45 \\
$\omega(\mathrm{deg})$ & 14 \\
$M(\mathrm{deg})$ & 210 \\
\hline
\end{tabular}

The initial attitude is defined with all Euler angles and rates set to zero.

Reflection and material properties of multi-layer insulations are based on [36]. The basic structure of MLI is composed of a single sheet of material, made of Polyethylene terephthalate (PET), thickness of $6 \mu \mathrm{m}$, and the aluminum coating is 1000 Á thick on both sides. This investigation assumes to be one layer of PET to simplify the model.

Two different forms of MLI are considered for the simulations: a flat sheet and a flat sheet which is folded along the middle at an angle of 90 degrees as in Fig. 2(a), dashed line. The dimensions of both configurations are: 1 meter of width and length, thickness of $6 \mu \mathrm{m}$. [37, 38]. The properties of the material are shown in Table 4 and this yields an area per mass ratio of: $119.90 \mathrm{~m}^{2} / \mathrm{kg}$.

Table 4. Properties of PET material.

\begin{tabular}{lllll}
\hline $\begin{array}{l}\text { Type } \\
\text { material }\end{array}$ & $\begin{array}{l}\text { Mass } \\
\text { Density } \\
{[\mathrm{kg} / \mathrm{m} 3]}\end{array}$ & $\begin{array}{l}\text { Young's } \\
\text { Modulus } \\
{[\mathrm{N} / \mathrm{m} 2]}\end{array}$ & $\begin{array}{l}\text { Poisson } \\
\text { 's ratio }\end{array}$ & $\mathrm{Cs}, \mathrm{Cd}, \mathrm{Ca}$ \\
\hline PET & 1,390 & $3.10 \times 10^{9}$ & 0.38 & 0.60 .260 .14 \\
\hline
\end{tabular}




\subsection{Deformation result validation}

To validate the FEM code for deformation of flexible material, we define that each feature consisting 2 elements, which have 3 nodes, red points, and apply force, $8.011 \mathrm{e}-7 \mathrm{~N}$, at the $1^{\text {st }}$ node, blue direction, following Fig. 27(a) and we define the boundary condition of degrees of freedom, nine degrees. We integrate the differential equations of motion with ODE45 function in MATLAB to study the deformation for 3 seconds. The results are shown in Fig. 27 and Fig. 28.

In Fig. 27(a), the $1^{\text {st }}$ node displacement moves to the right side while the $3^{\text {rd }}$ node move downwards. The $1^{\text {st }}$ node of the flat plate in Fig. 28(a) moves down and results pulls with it the $2^{\text {nd }}$ and $3^{\text {nd }}$ nodes due to tension forces. These results are used to validate the simple model of the natural displacements of low weight and thin material. Due to the deformation in Fig. 27(b) and Fig. 28(b), the center of mass moves from its initial position. As a result, it affects the attitude motion of the debris as shown in the next section.
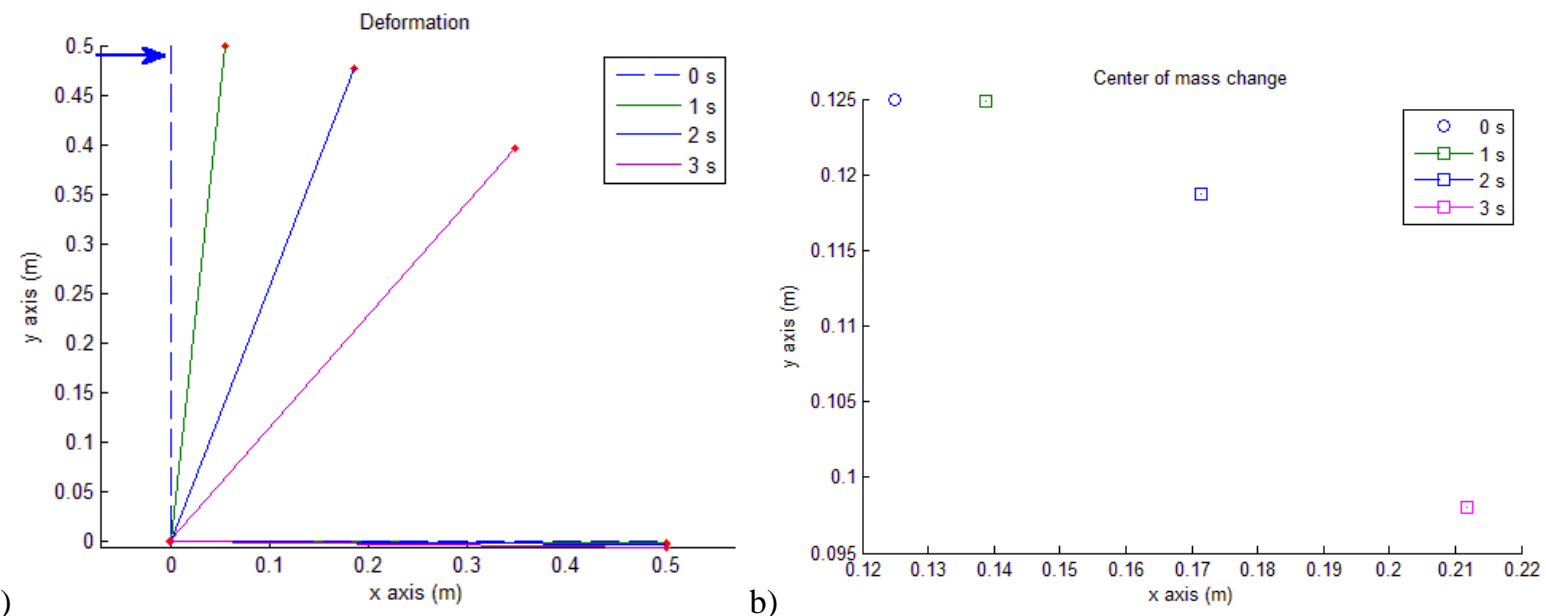

a)

Fig. 27 a) Deformation of folded flat plate at $0,1,2$ and $3 \mathrm{~s}$ by force $8.011 \mathrm{e}-7 \mathrm{~N}$ in the first node in right direction $b$ ) The change of center of mass of folded flat plate at $0,1,2$ and $3 \mathrm{~s}$

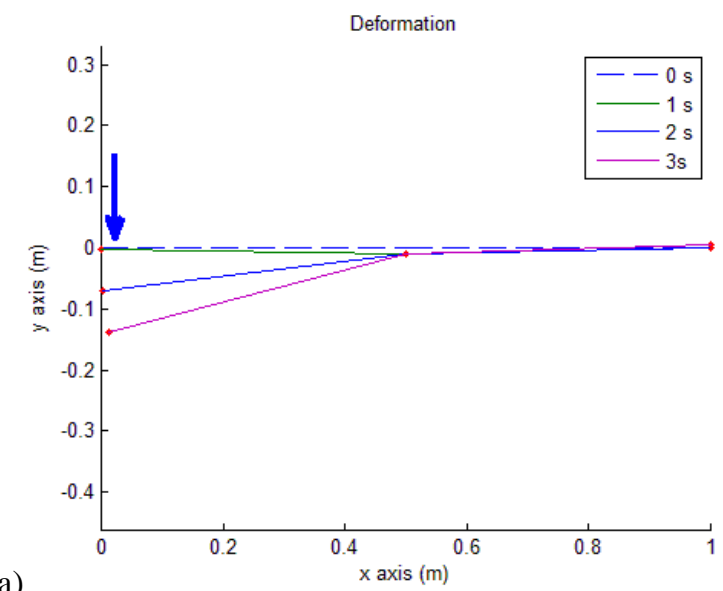

a)

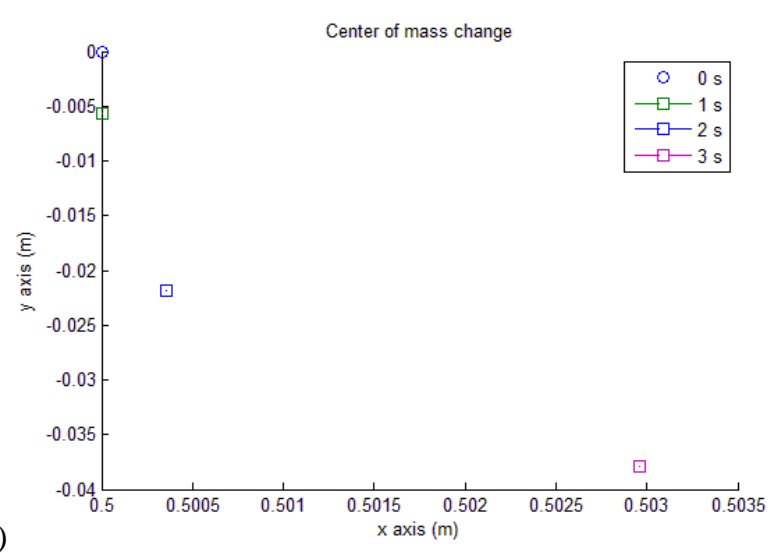

Fig. 28 a) Deformation of flat plate in $0.2,0.4$ and $0.6 \mathrm{~s}$ due to a force of $8.011 \mathrm{e}-7 \mathrm{~N}$ in the first node in $-\mathrm{y}$ direction b) The change of center of mass of flat plate 


\subsection{Results}

The Earth gravitation, third body from the Sun and Moon and SRP radiation pressure have been applied to four different configurations characteristics, rigid flat sheet, flexible flat sheet, rigid folded plate and flexible folded plate. The evolution of eccentricity and inclination over 3 days are shown in Fig. 29 and Fig. 30 while a long term propagation for over 150 days are shown in Fig. 31 and Fig. 32. In Fig. 29 (a), eccentricity of rigid body grows up consistently while those of flexible body are different that fluctuate in same peak of range and inclination and it is very obvious in Fig. 29(b) to see inconsistency of flexible debris of both initial geometry of flat plate and folded plate. In Fig. 30(a), they have similar trend to increase but the flexible debris are below that of the rigid body in Fig. 30(b). To monitor in the long period, the mean eccentricity of the orbit of the rigid body increases (Fig. 31) while in the case of flexible debris do not rise significantly. The mean inclination for the rigid debris in Fig. 32 increases while for flexible debris it oscillates around a mean value. The reason of this can be found in the change of the crosssectional area due to the deformation of the sheet, effectively changing exposed area to solar radiation pressure and sun angles.
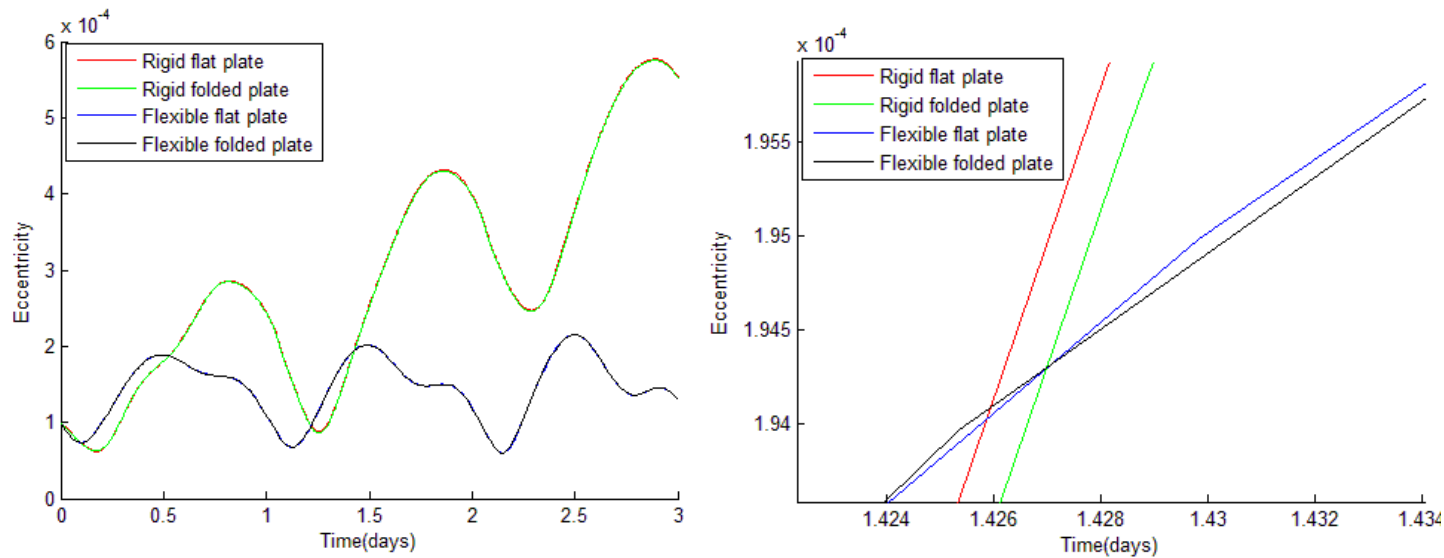

Fig. 29. a) Eccentricity of flat plate and folded up flat plate in both rigid and flexible cases b) Magnify eccentricity period of $1.42-1.44$ days
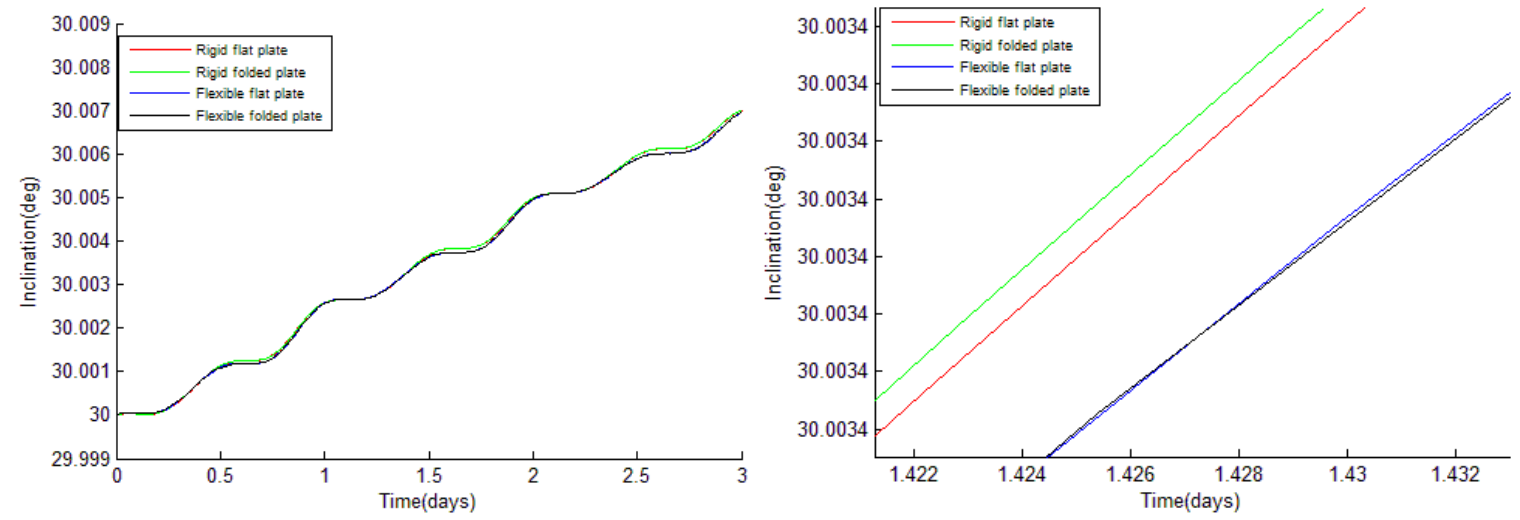

Fig. 30. a) Inclination of flat plate and folded up flat plate in both rigid and flexible cases b) Magnify inclination period of 1.42-1.43 days 

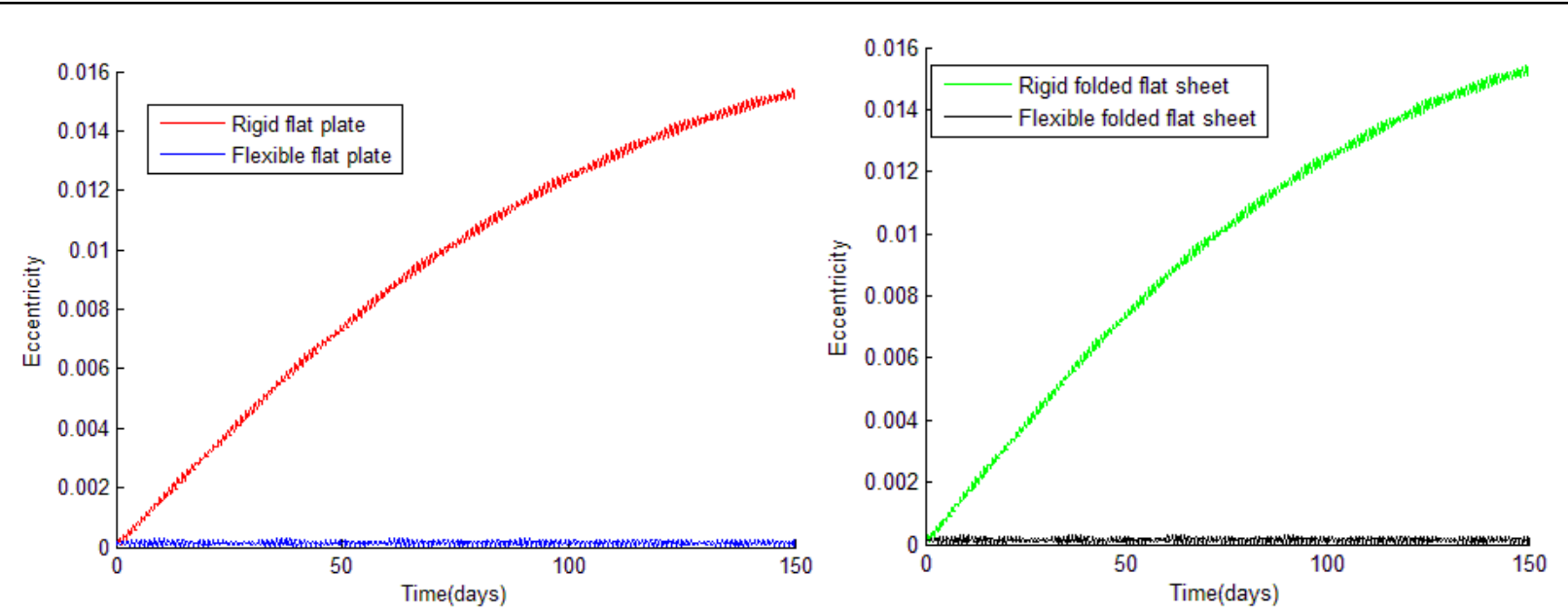

Fig. 31. a) Eccentricity of flat plate in both rigid and flexible cases in $\mathbf{1 5 0}$ days b) Eccentricity of folded flat plate in both rigid and flexible cases in 150 days
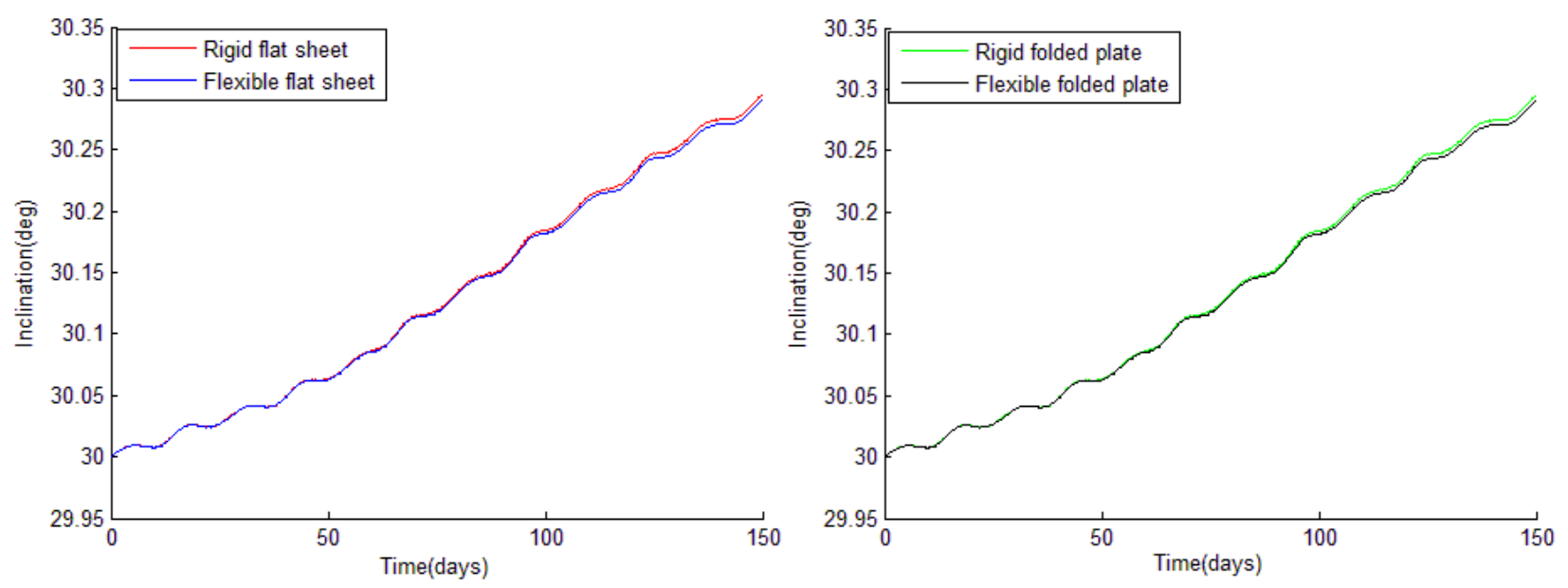

Fig. 32. a) Inclination of flat plate in both rigid and flexible cases in 150 days b) Inclination of folded flat plate in both rigid and flexible debris in 150 days

In Fig. 33 and Fig. 34, the attitude of the flexible debris in both configurations appears to rotate significantly slower than for the rigid debris for which the flat configuration presents the fastest displacements. As a result of deformation, the center of mass and moments of inertia are always changing, leading to continuously varying effects from SRP and gravitational torques. After propagating over 150 days in Fig. 35, Fig. 36, Fig. 37 and Fig. 38, the flexible debris, shows periods of small changes alternating with periods of high fluctuations. This effect does not occur for the rigid debris which exhibits a more uniform behavior in time. 

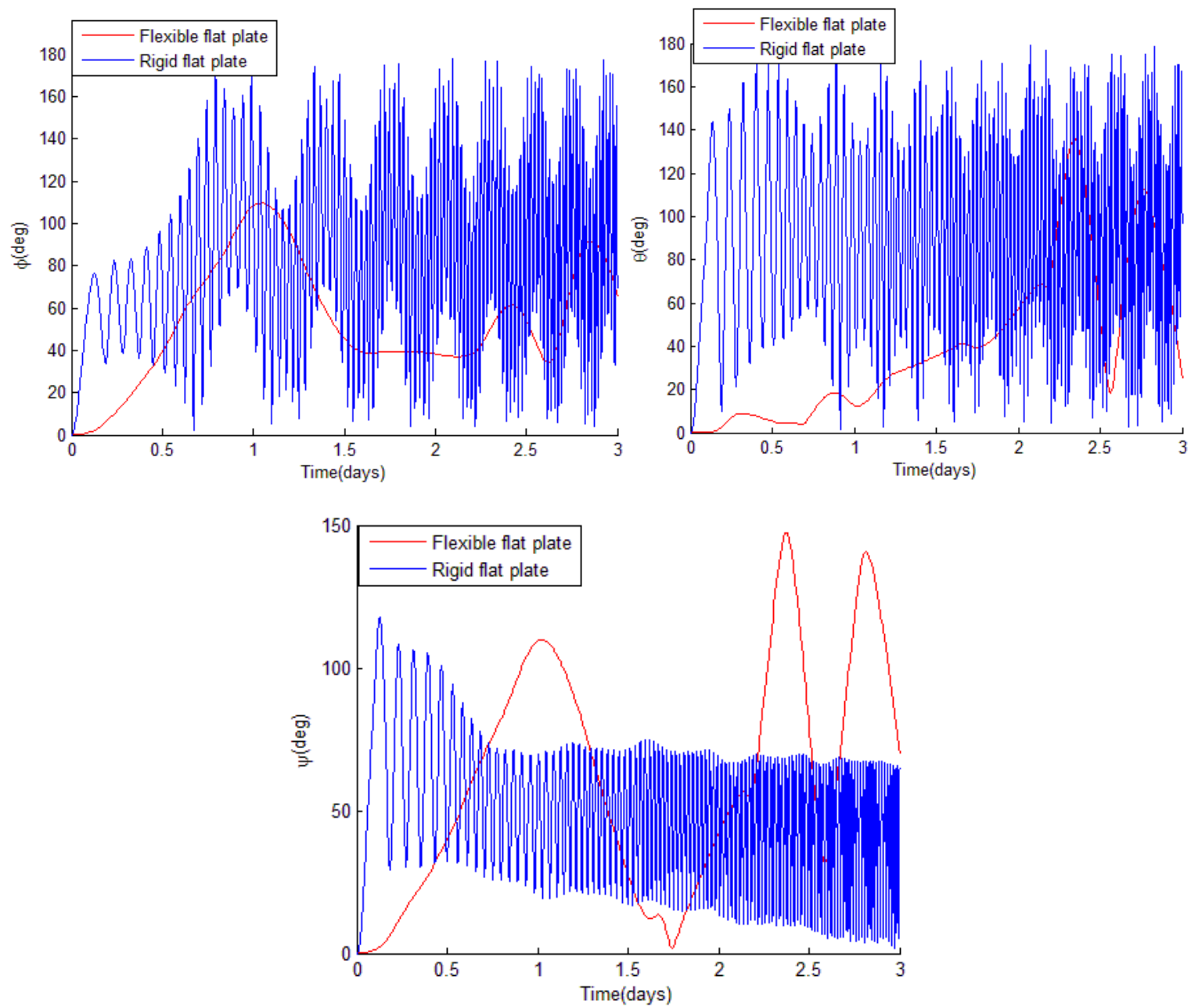

Fig. 33. Euler angles of flat plate in case of rigid body and flexible body in 3 days
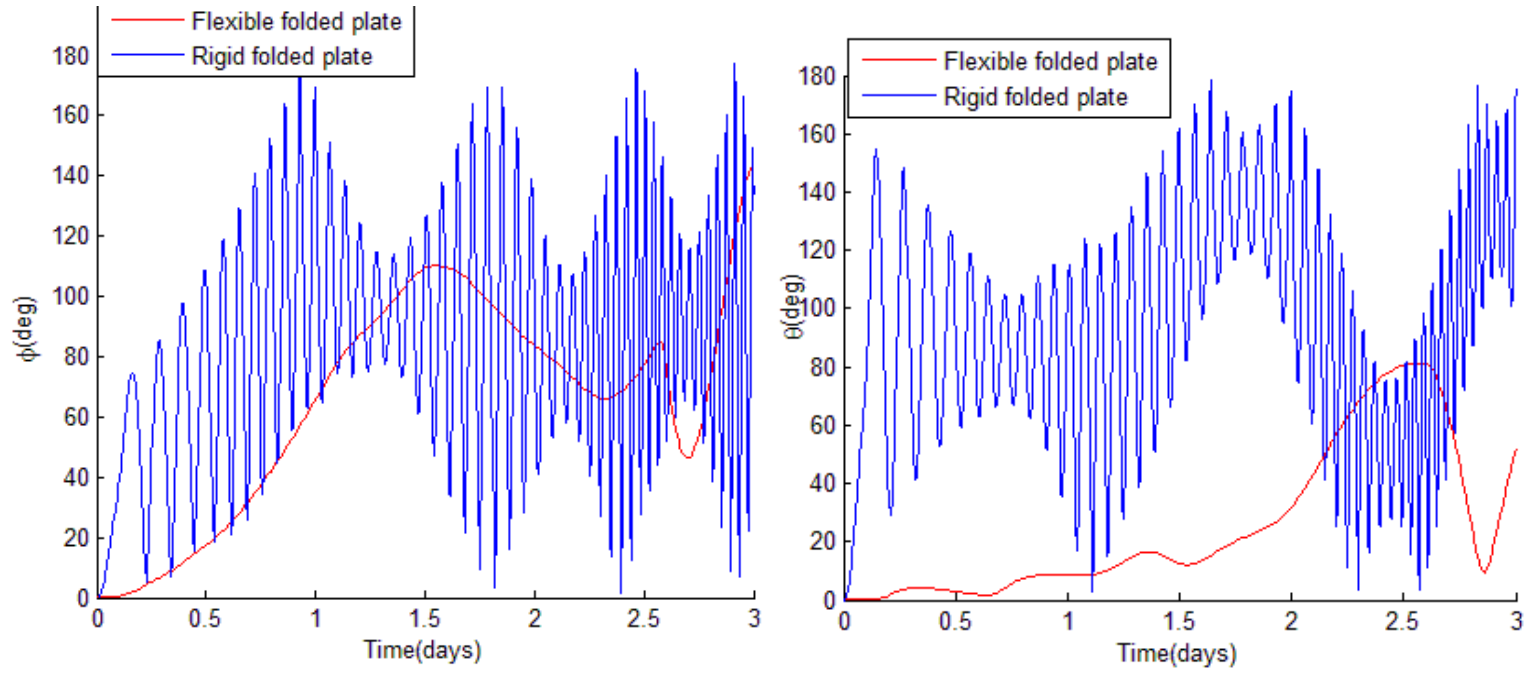


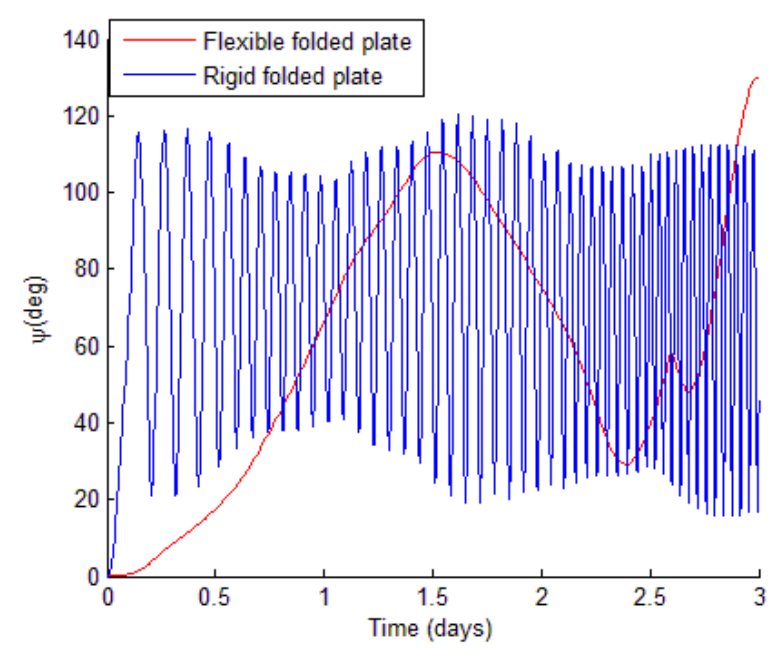

Fig. 34. Euler angles of folded flat sheet in case of rigid body and flexible body in $\mathbf{3}$ days
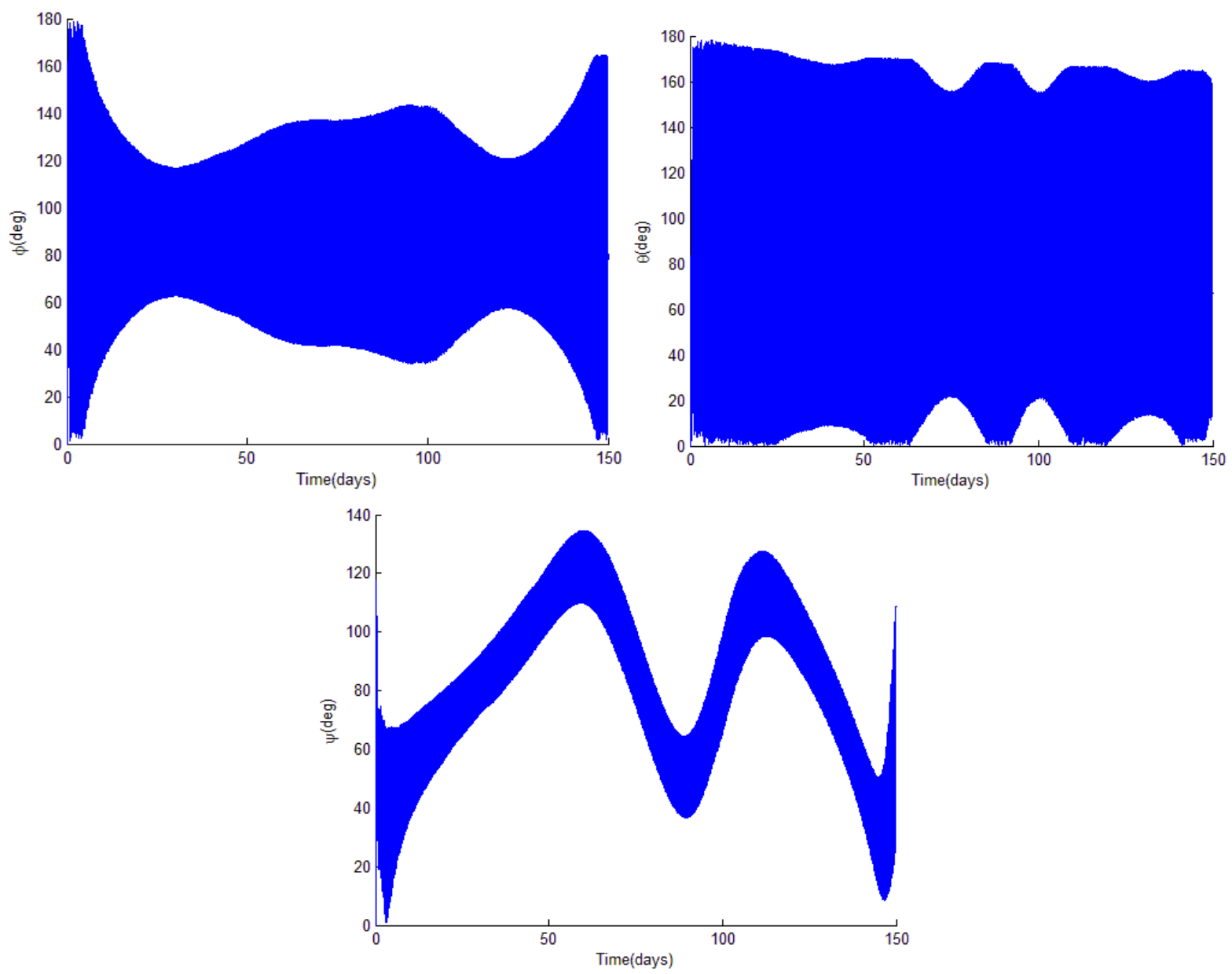

Fig. 35. Euler angles of rigid flat plate in 150 days 

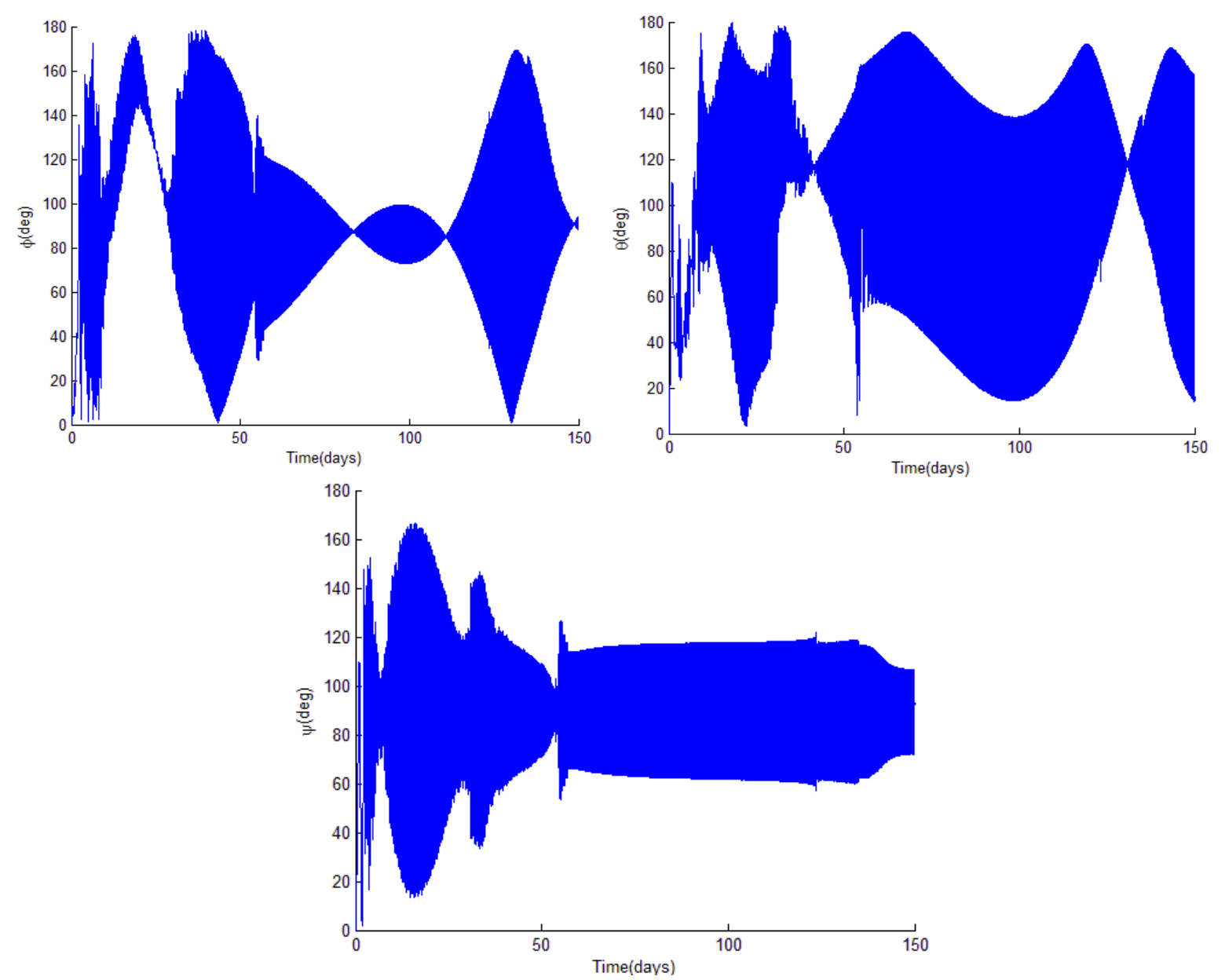

Fig. 36. Euler angles of flexible flat plate in 150 days 

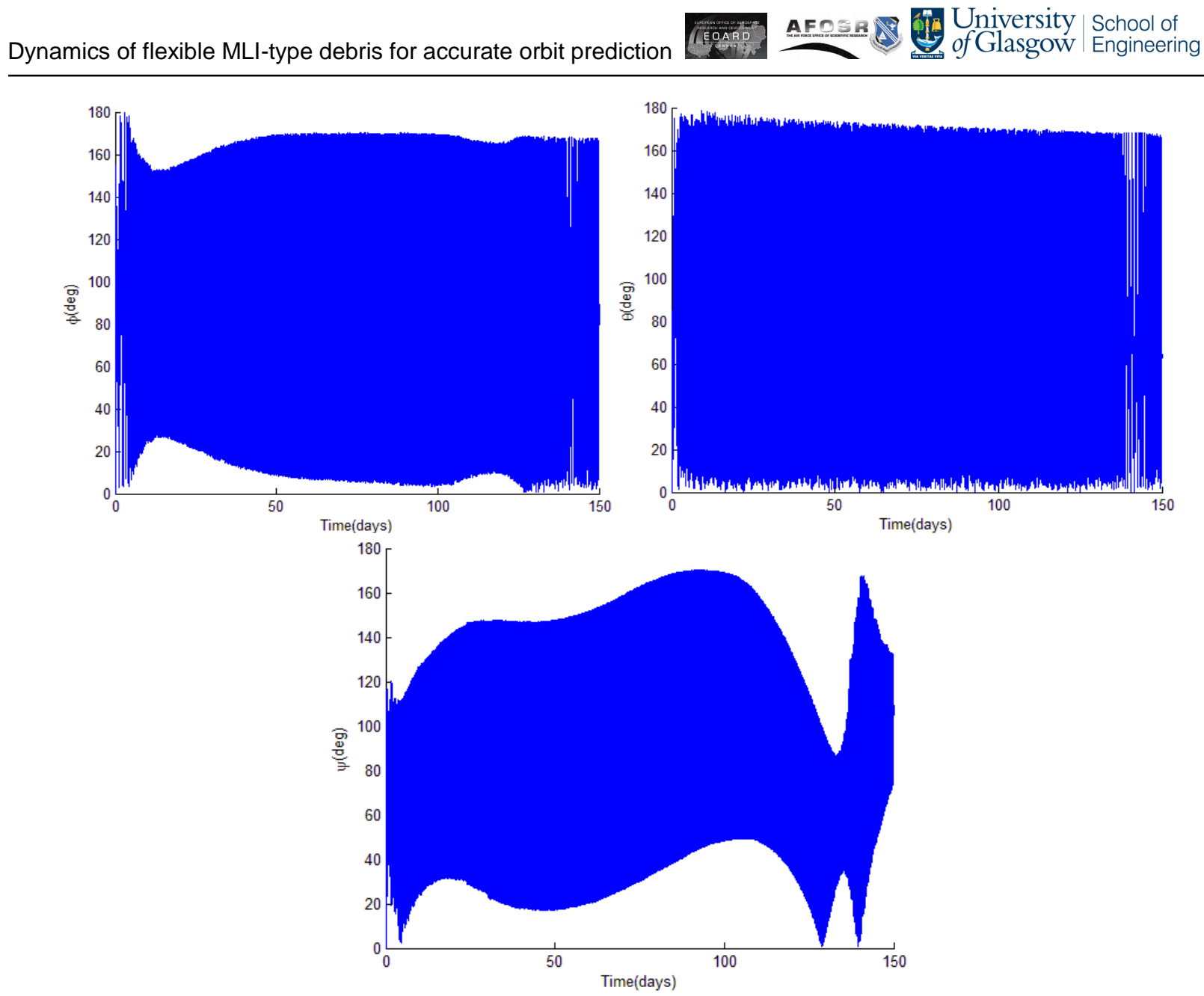

Fig. 37. Euler angles of rigid folded flat plate in 150 days 

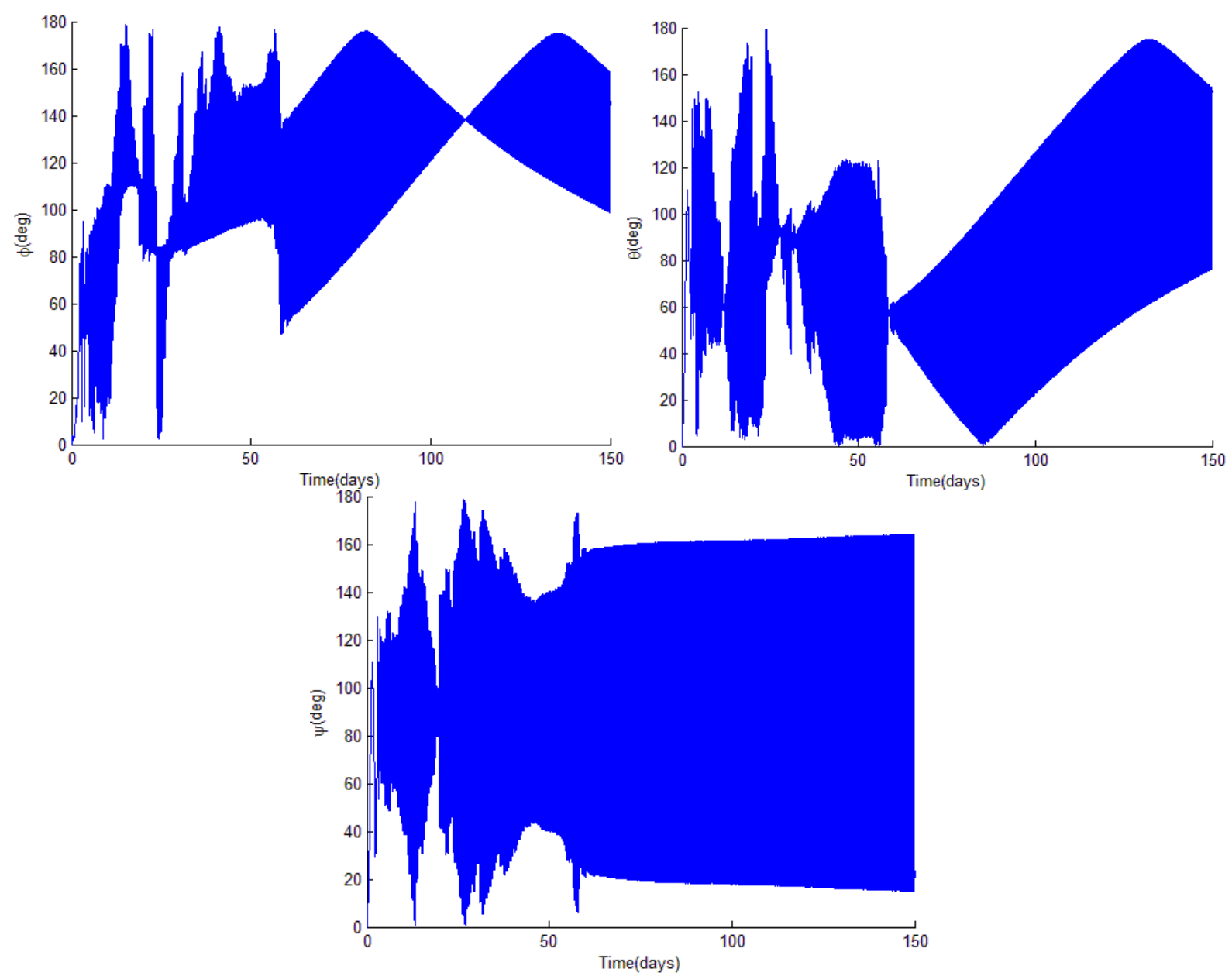

Fig. 38. Euler angles of flexible folded flat plate in 150 days

\subsection{Conclusion}

This section investigated the attitude and orbital debris of flexible debris in geostationary region in long term period 150 days. The flat plate and folded flat plate have been simulated in rigid and flexible cases by using same properties of PET.

In a propagation of 150 days, some differences can be noticed between the rigid and the flexible cases. The deformation implies change in the cross-sectional area exposed to the sun, as well as the center of mass and pressure, and moments of inertia.

From this investigation, when an accurate model of the flexible debris is provided, we can improve to the precision of prediction for the orbit determination, the orbital life time and also investigate the material types of the debris from rotation rates.

Finally, we underline that investigation did not consider large deformations, for which non-linear methods would be required to increase the accuracy of the models for orbital and attitude prediction. In addition, a higher-order propagator shall be used to decrease the simulation time and increase the accuracy in longer periods. 


\section{Multi-body deformation model}

The previous approach implied the strong assumption that deformations are small. While in principle the approach can be applied to arbitrarily large deformations, the results are less and less accurate, the larger the deformations are. Because of the thin membranes that are modelled in this work, it is expected that large displacements might well be possible.

For this reason, we introduce here an approach that aims at modelling a flexible thin sheet with full, non-linear equations of motion. In particular, the debris object is modelled with a number of lump masses, each representing a section of the thin membrane, inter-linked ideal tethers. Suitable constraints will be set in place, to allow the deformation of the structure, as well as modelling the internal forces that arise due to the deformation (elastic and viscous). In addition, we will consider self-shadowing effects on the membrane.

\subsection{Model and dynamics}

A novel model for a thin, highly flexible MLI-type membrane is proposed here, in terms of multi-body dynamics, and it is solved using fundamental Newtonian mechanics. Assuming that the membrane folds along one line, the deformation can be studied as a two-dimensional problem (see Fig. 39 (a)). The membrane is modelled as a series of lump masses, interconnected with rigid rods. The masses act as rotational joints for the plates and include rotational spring and damper to simulate the bending stiffness of the membrane. The membrane is free to move and tumble in the three dimensional space, however the folding lines are always the same on the membrane, and parallel to each other, allowing to describe the deformation in a two-dimensional plane perpendicular to the folding lines. In this work, only three lump masses and one folding line are considered. This simplified model, in undeformed (flat) and folded states is illustrated in Fig. 39 (c) and (c) respectively. Representation in 3D space and reference angles are in Fig. 40 and Fig. 41. 

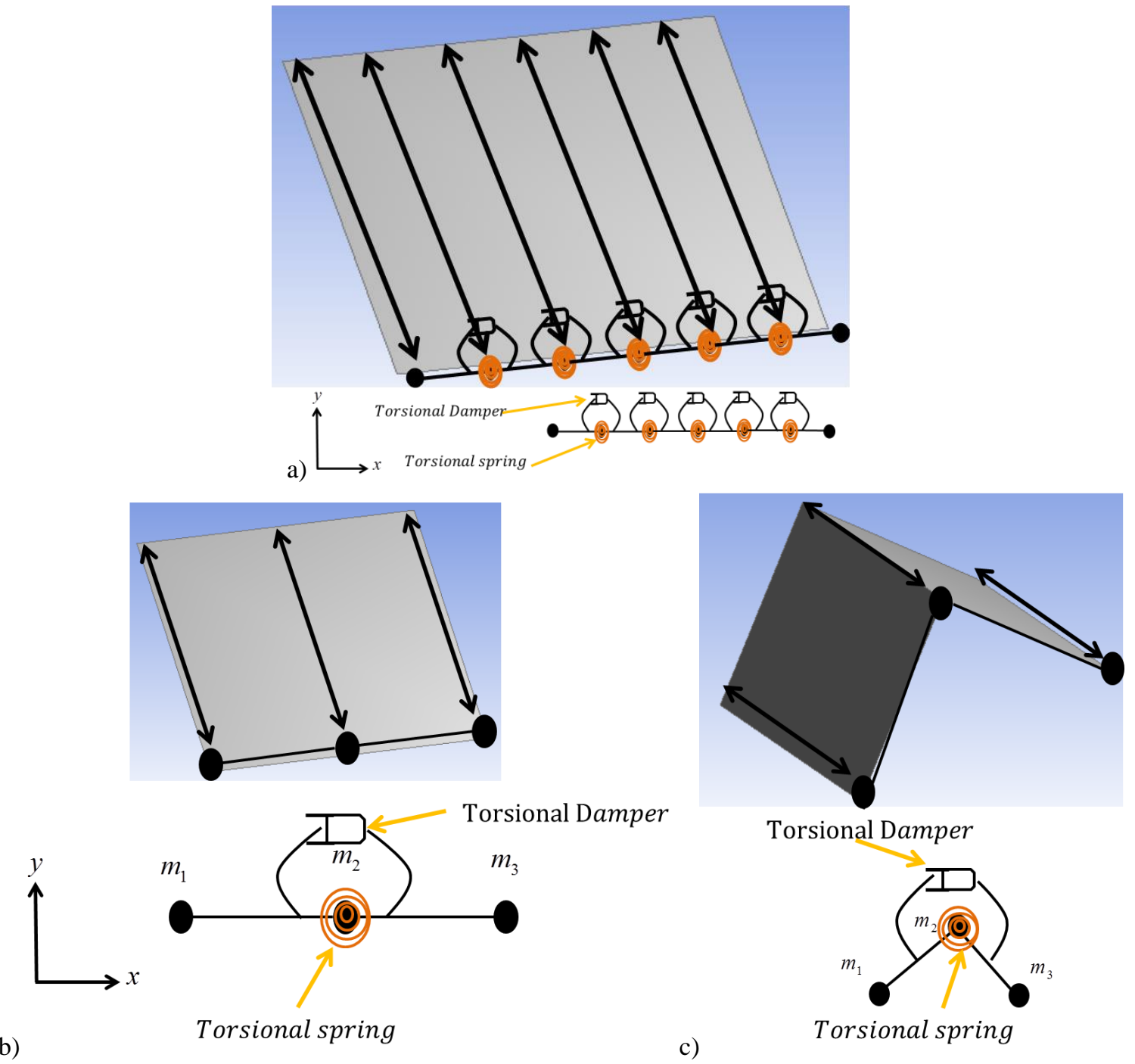

c)

Torsional spring

Fig. 39. a) The membrane for $2 \mathrm{D}$ deformation is modeled with series of masses including torsional springs and dampers. b) Membrane completely flat; c) Membrane folded along one folding line. The torsional spring in the model simulates the membrane bending stiffness. 


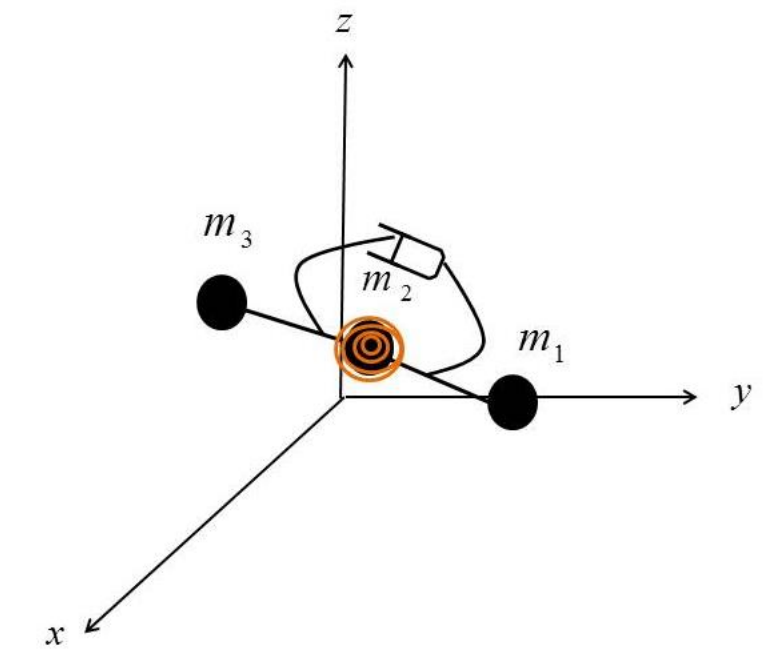

a)

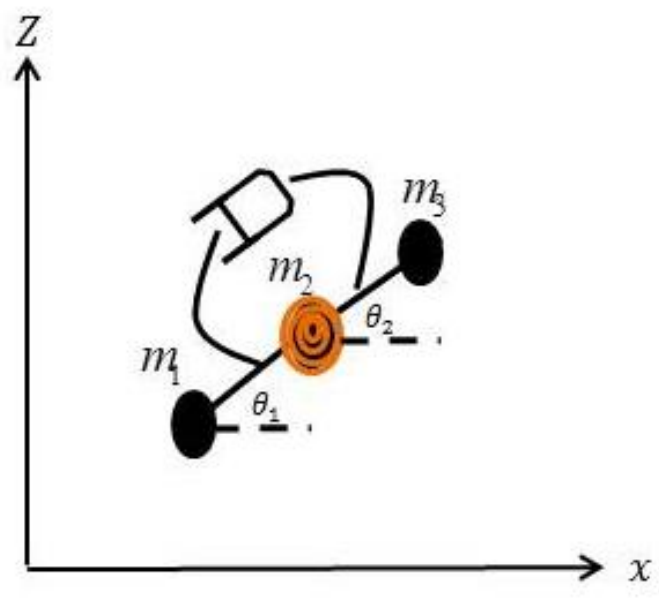

b)

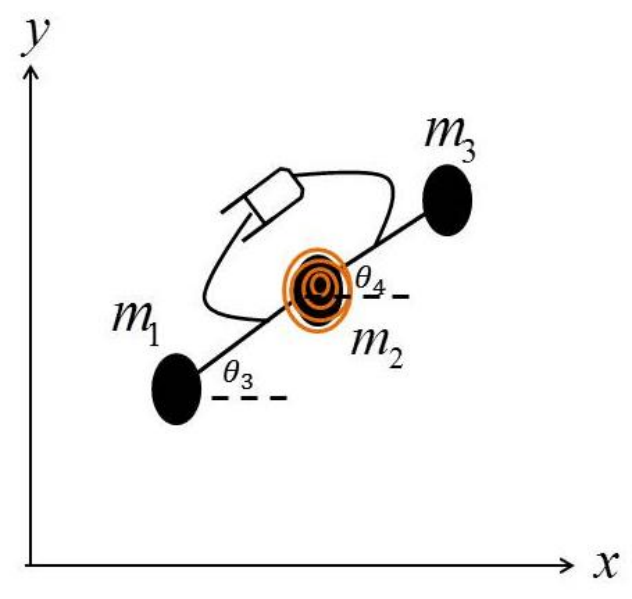

c)

Fig. 40. a) the flexible model in three dimensions and inertial frame $b$ ) the model in $x-z$ view c) the model in $x$ y view.

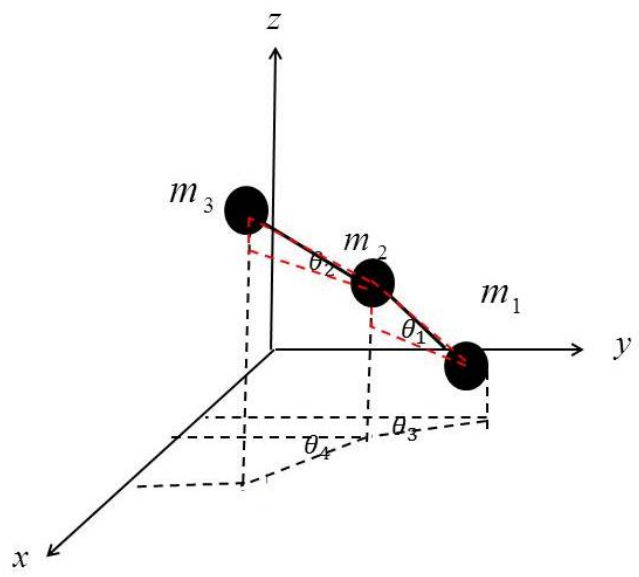

Fig. 41. Angles defining the direction of the imaginary rods with respect to an inertial frame. 
In order to write the differential equations of motion for this system of masses, we imagine to cut the rods and we introduce at each end the axial reaction forces produced by the rods on the masses, as in Fig. 42.
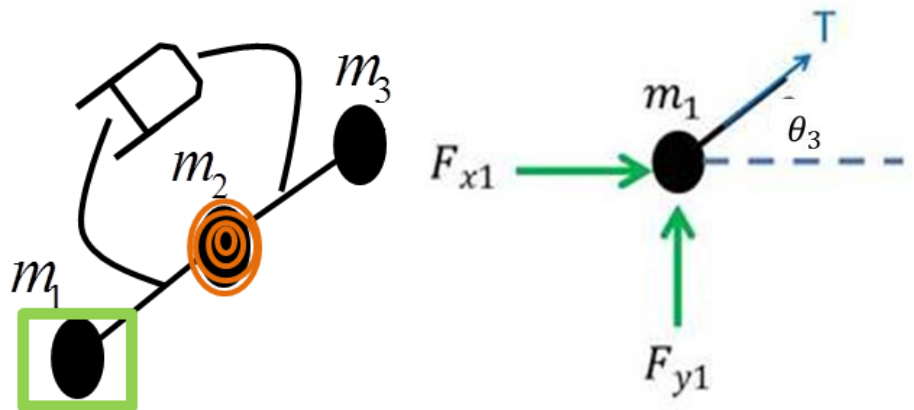

a)
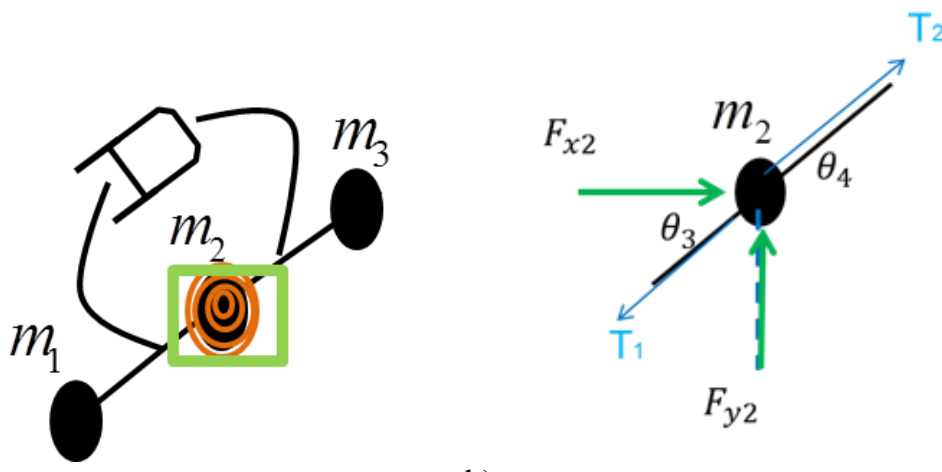

b)
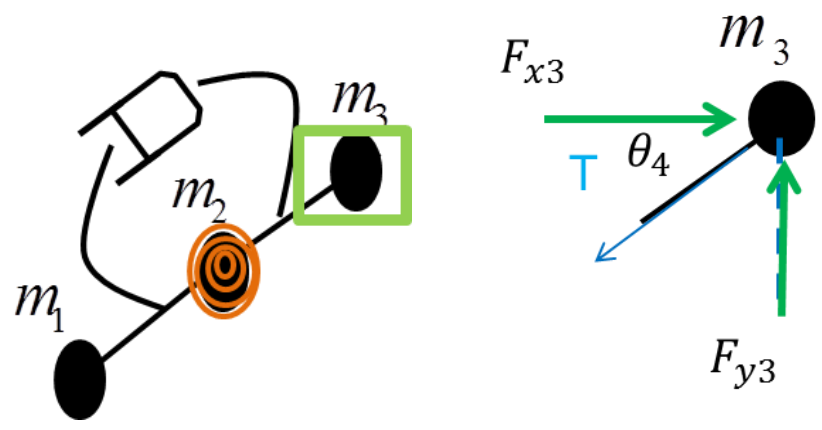

c)

Fig. 42. Free body diagram of each lump mass a) $1^{\text {st }}$ free body diagram b) $2^{\text {nd }}$ free body diagram c) $3^{\text {rd }}$ free body diagram

Now, the equation of motion for each of the three masses $i=1,2,3$ is:

$$
\begin{gathered}
\vec{F}_{\text {total }, i}+\vec{T}_{i}=m_{i} \ddot{x_{i}} \\
\ddot{x_{i}}=\frac{\vec{F}_{\text {total }, i}+T_{i}}{m_{i}}
\end{gathered}
$$

$F_{\text {total }, i}$ is total force acting on lump masses $\left(F_{t o t a l, i}=F_{e x t}+F_{s, i}+F_{d, i}\right) . F_{e x t}$ is the external force from conservative and non-conservative perturbations (J2, SRP and third body from the Sun and the Moon), $T_{i}$ is the tension force generated by the rod, $F_{s, i}$ is the rotational spring force of $\mathrm{i}^{\text {th }}$ mass, $F_{d, i}$ is the rotational damper force, $\ddot{\bar{x}}_{i}$ is total three 
axis acceleration of $\mathrm{i}^{\text {th }}$ lump mass $(\mathrm{J} 2$, SRP and third body acceleration). The rotational torques of the spring and the damper are found from their corresponding torques, acting at mass 2, between the first and the second rod (Fig. 43).
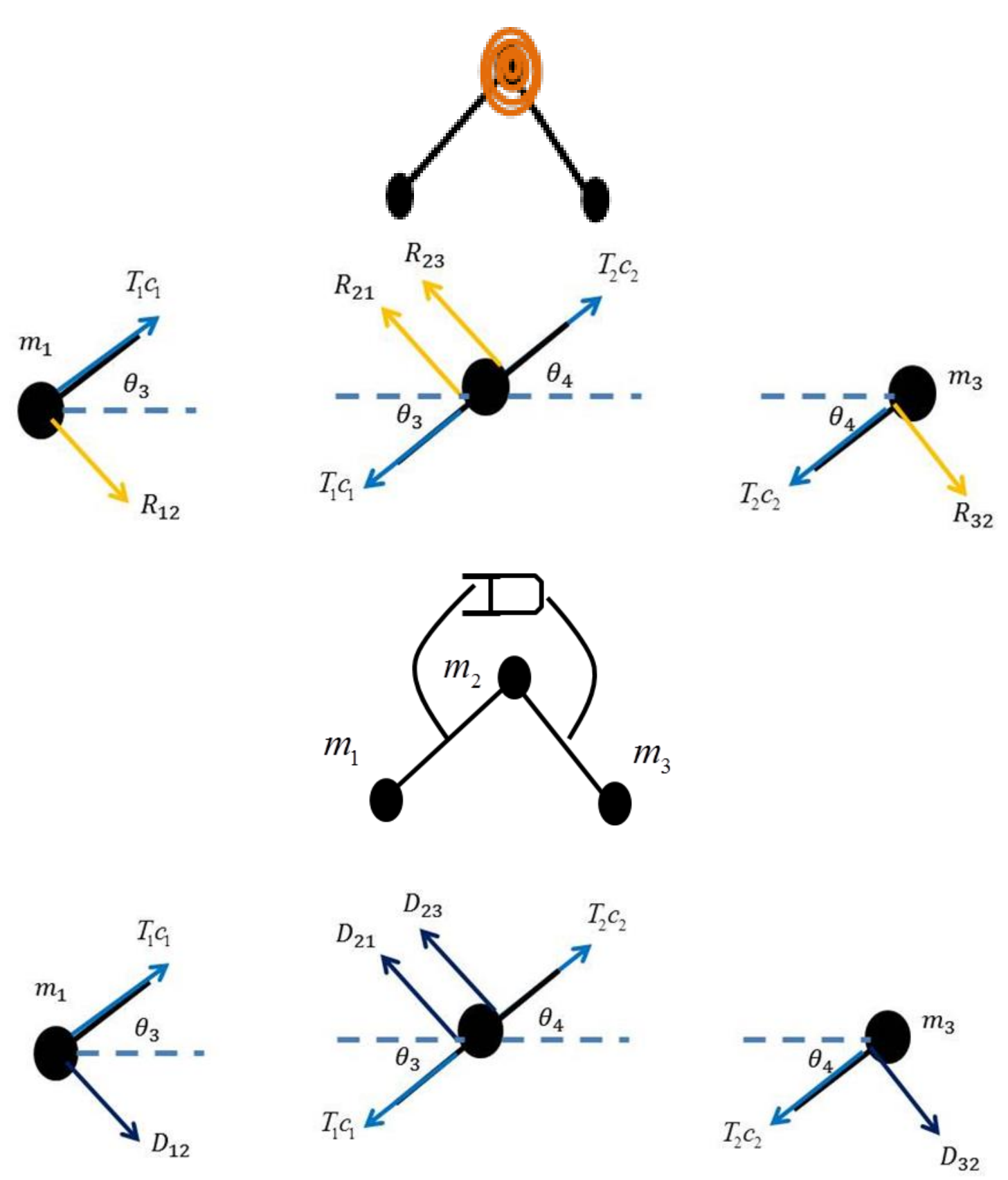

b)

Fig. 43. Free body diagram of each lump mass a) Forces from torsional spring b) Forces from torsional damper

The rotational spring force is:

$$
F_{s}=k \theta
$$




$$
k=\frac{E I}{L}
$$

Where $k$ is rotational spring constant, $\theta$ is angle of deformation, $E$ is young modulus, $I$ is the moment of inertia of thin plate, $L$ is the length of each rod.

The rotational damping force is:

$$
\begin{aligned}
& F_{d}=c \dot{\theta} \\
& c=D F \sqrt{M k_{s}}
\end{aligned}
$$

Where ${ }_{c}$ is the rotational damping constant, $\dot{\theta}$ is angular velocity of the deformation, $D F$ is dissipation factor of material [39], $M$ is total mass of membrane.

To be able to integrate the equations of motion (2), the rod tensions $\mathrm{T}$ is still to be determined. To do that, we exploit the constraint that each rod is infinitely rigid in the axial direction, and thus (length of connecting rods, $l_{i}$ ):

$$
\left(x_{i+1}-x_{i}\right)^{2}+\left(y_{i+1}-y_{i}\right)^{2}+\left(z_{i+1}-z_{i}\right)^{2}=l_{i}^{2}
$$

Taking the second differential of these two equations we get:

$$
2\left(\ddot{x}_{i}-\ddot{x}_{i+1}\right)\left(x_{i}-x_{i+1}\right)+2\left(\ddot{y}_{i}-\ddot{y}_{i+1}\right)\left(y_{i}-y_{i+1}\right)+2\left(\ddot{z}_{i}-\ddot{z}_{i+1}\right)\left(z_{i}-z_{i+1}\right)+2\left(\dot{x}_{i}-\dot{x}_{i+1}\right)^{2}+2\left(\dot{y}_{i}-\dot{y}_{i+1}\right)^{2}+2\left(\dot{z}_{i}-\dot{z}_{i+1}\right)^{2}=0
$$

In these equations, positions are velocities are known from the state vector of the system. The accelerations are unknown, however an expression of them is in Eqs. (2). Substituting, both tensions T appear in the equations, which can then be written as a linear system:

$$
\left[\begin{array}{ll}
C_{1} & C_{2} \\
C_{3} & C_{4}
\end{array}\right\rfloor\left[\begin{array}{l}
T_{1} \\
T_{2}
\end{array}\right]=\left[\begin{array}{l}
A_{1} \\
A_{2}
\end{array}\right]
$$

Where:

$$
\begin{aligned}
& C_{1}=c_{1} c_{3}\left(x_{1}-x_{2}\right)\left(\frac{1}{m_{1}}+\frac{1}{m_{2}}\right)+c_{1} s_{3}\left(y_{1}-y_{2}\right)\left(\frac{1}{m_{1}}+\frac{1}{m_{2}}\right)+s_{1}\left(z_{1}-z_{2}\right)\left(\frac{1}{m_{1}}+\frac{1}{m_{2}}\right) \\
& C_{2}=-\frac{c_{2} c_{4}}{m_{2}}\left(x_{1}-x_{2}\right)-\frac{c_{2} s_{4}}{m_{2}}\left(y_{1}-y_{2}\right)-\frac{s_{2}}{m_{2}}\left(z_{1}-z_{2}\right) \\
& C_{3}=-\frac{c_{1} c_{3}}{m_{2}}\left(x_{2}-x_{3}\right)-\frac{c_{1} s_{3}}{m_{2}}\left(y_{2}-y_{3}\right)+\frac{s_{1}}{m_{3}}\left(z_{2}-z_{3}\right) \\
& C_{4}=c_{2} c_{4}\left(x_{2}-x_{3}\right)\left(\frac{1}{m_{2}}+\frac{1}{m_{3}}\right)+c_{2} s_{2}\left(y_{2}-y_{3}\right)\left(\frac{1}{m_{2}}+\frac{1}{m_{3}}\right)+s_{3}\left(z_{2}-z_{3}\right)\left(\frac{1}{m_{2}}+\frac{1}{m_{3}}\right) \\
& A_{1}=-\left(\dot{x}_{1}-\dot{x}_{2}\right)^{2}-\left(\dot{y}_{1}-\dot{y}_{2}\right)^{2}-\left(\dot{z}_{1}-\dot{z}_{2}\right)^{2}-\frac{F_{x 1}}{m_{1}}\left(x_{1}-x_{2}\right)+\frac{F_{x 2}}{m_{1}}\left(x_{1}-x_{2}\right)-\frac{F_{y 1}}{m_{1}}\left(y_{1}-y_{2}\right)+\frac{F_{y 2}}{m_{2}}\left(y_{1}-y_{2}\right)-\frac{F_{z 1}}{m_{1}}\left(z_{1}-z_{2}\right)+\frac{F_{z 2}}{m_{2}}\left(z_{1}-z_{2}\right)-F_{s}-F_{d} \\
& A_{2}=-\left(\dot{x}_{2}-\dot{x}_{3}\right)^{2}-\left(\dot{y}_{2}-\dot{y}_{3}\right)^{2}-\left(\dot{z}_{2}-\dot{z}_{3}\right)^{2}-\frac{F_{x 2}}{m_{2}}\left(x_{2}-x_{3}\right)+\frac{F_{x 3}}{m_{3}}\left(x_{2}-x_{3}\right)-\frac{F_{y 2}}{m_{2}}\left(y_{2}-y_{3}\right)+\frac{F_{y 3}}{m_{3}}\left(y_{2}-y_{3}\right)-\frac{F_{z 2}}{m_{2}}\left(z_{2}-z_{3}\right)+\frac{F_{z 3}}{m_{3}}\left(z_{2}-z_{3}\right)-F_{s}-F_{d} \\
& s_{1}=\sin \left(\theta_{1}\right) \quad s_{3}=\sin \left(\theta_{3}\right) \\
& c_{1}=\cos \left(\theta_{1}\right) \quad \text { and } c_{3}=\cos \left(\theta_{3}\right) \\
& s_{2}=\sin \left(\theta_{2}\right) \\
& c_{2}=\cos \left(\theta_{2}\right) \quad R_{12}=-R_{21} \text { and } R_{23}=-R_{32} \\
& c_{4}=\cos \left(\theta_{4}\right)
\end{aligned}
$$

The tensions can then be calculated by inverting the system: 


$$
\left[\begin{array}{l}
T_{1} \\
T_{2}
\end{array}\right]=\left[\begin{array}{ll}
C_{1} & C_{2} \\
C_{3} & C_{4}
\end{array}\right]^{-1}\left[\begin{array}{l}
A_{1} \\
A_{2}
\end{array}\right]
$$

Substituting these values in Eqs. (2) allows to integrate them over time, to determine the position and velocity of each lump mass.

\subsubsection{Numerical validation}

To test the spring and damper of the model, it is required to set a non-flat geometry as initial condition (triangular shape in Fig. 44). The oscillation in Fig. 45 (b) is forced by the rotational spring only. The damper slowly reduces the amplitude of the oscillation, until it comes to a complete stop, as in Fig. 46.

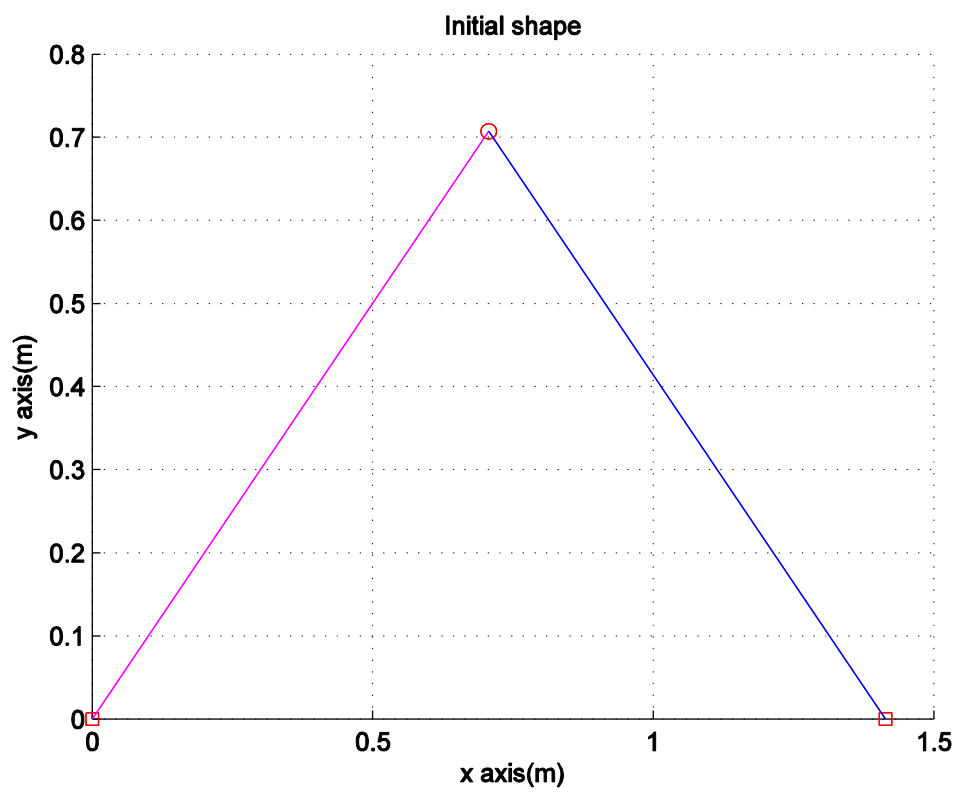

Fig. 44. Initial shape 

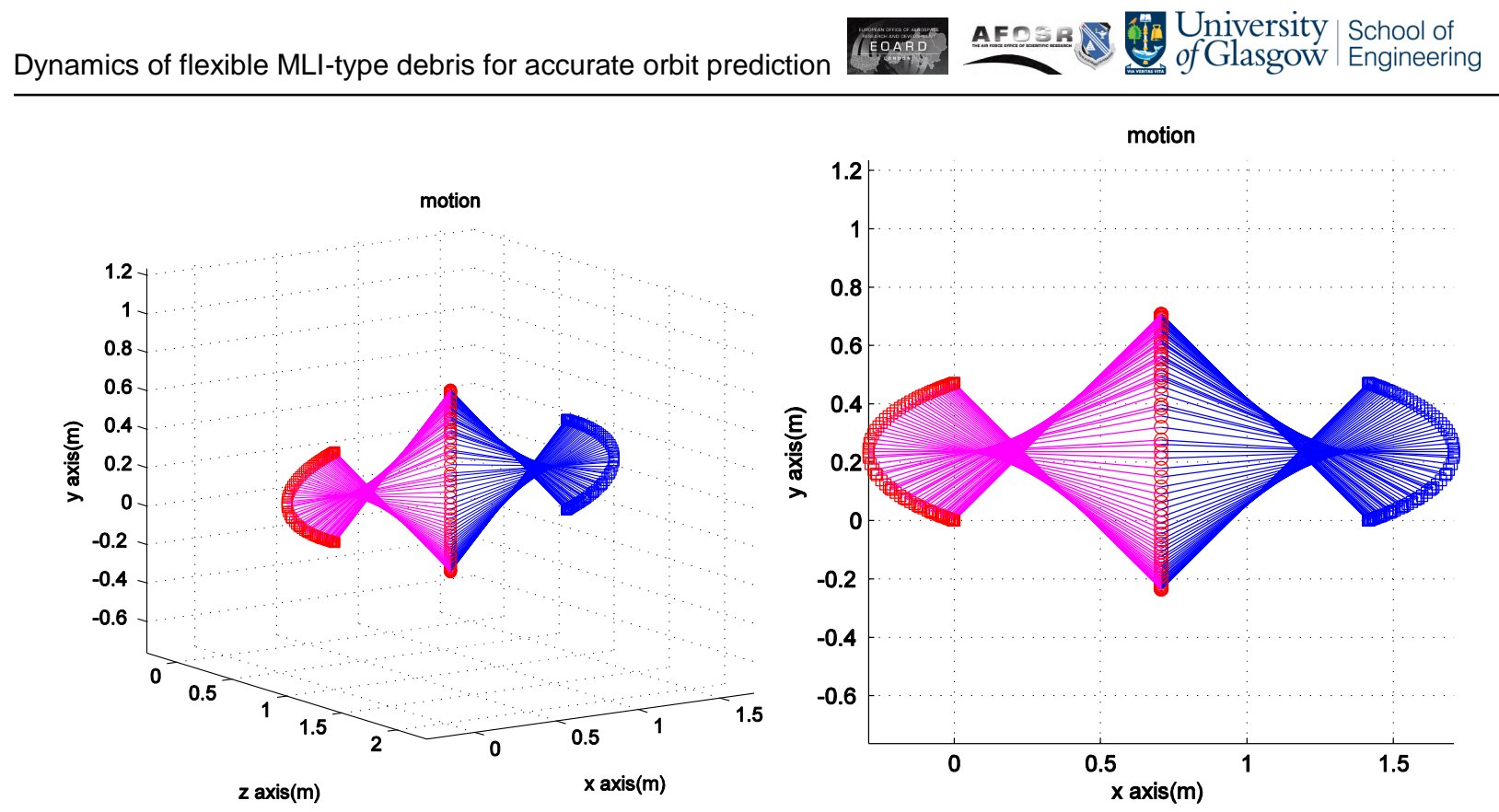

Fig. 45. The oscillation due to deformation from non-equilibrium results from torsional spring without external force

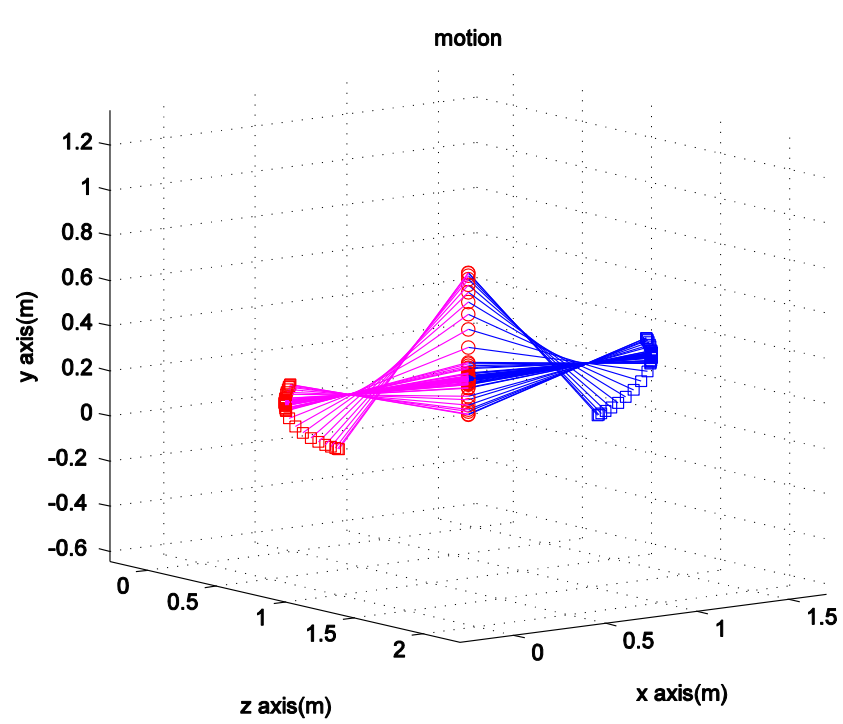

a)

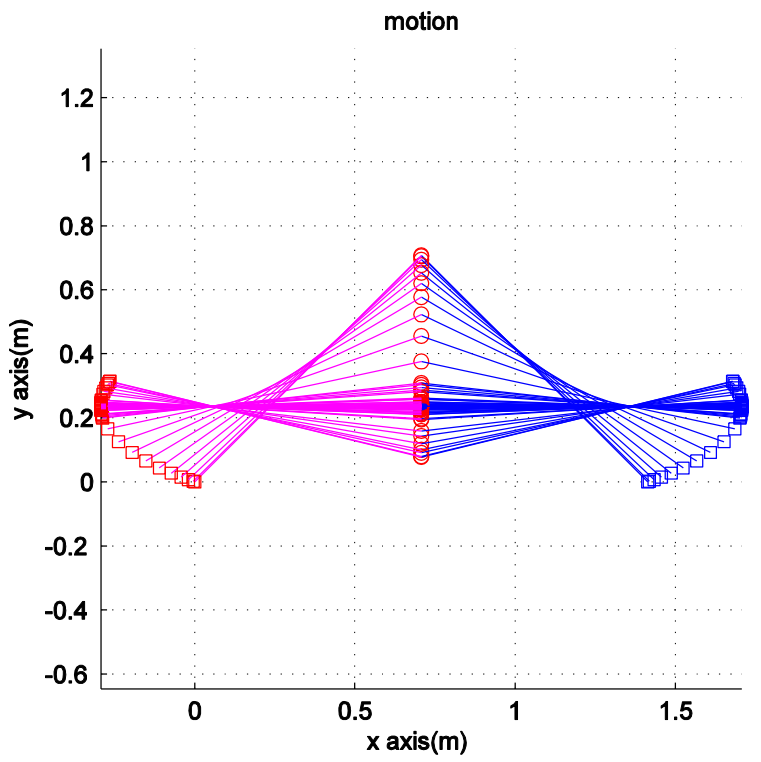

b)

Fig. 46. Torsional spring and torsional damper test show that oscillate and back to equilibrium due to torsional damper.

\subsubsection{Self shadowing}

The planar shadow projection, first developed by Blinn [40], allows shadows to be cast on a planar surface as in Fig. 47. The point $\vec{p}$ is the projection of vertex $\vec{v}$ onto a plane $\mathrm{P}: \vec{n} \cdot x+d=0$ due to a location of light source $\bar{l}$. The plane $\mathrm{P}$ can be described as

$$
\vec{p}=\vec{l}-\frac{d+\bar{n} \cdot \vec{l}}{n \cdot(v-l)}(\vec{v}-\vec{l})
$$


This can be written into a projection matrix $(\mathrm{Mv}=\mathrm{p})$ [41].

$$
M=\left[\begin{array}{cccc}
n . l+d-l_{x} n_{x} & -l_{x} n_{y} & -l_{x} n_{z} & -l_{x} d \\
-l_{y} n_{x} & n . l+d-l_{y} n_{y} & -l_{y} n_{z} & -l_{y} d \\
-l_{z} n_{x} & -l_{z} n_{y} & n . l+d-l_{z} n_{z} & -l_{z} d \\
-n_{x} & -n_{y} & -n_{z} & n . l
\end{array}\right]
$$

The self-shadowing algorithm has two main steps. Firstly, when two planes are oriented in space, it is important to check which plane casts shadows on the other. The vector from the Sun to the centre of mass on a panel will determine which face is closer. Then, the algorithm determined if an area of the panel is exposed to SRP by checking the intersection of area between the shadow and the panel. This algorithm is calculated on each step of integration.

Fig. 47(a) presents a self-shadowing simulation. The panel above (grey) acts as a shading panel. The bottom panel (brown) has a shadow cast upon its whole surface. The shaded area depends on the location of the light source and the model's shape. Fig. 47 (b) shows different shadows created by moving the light source position and Fig. 47 (c) by moving both light position and changing orientation.
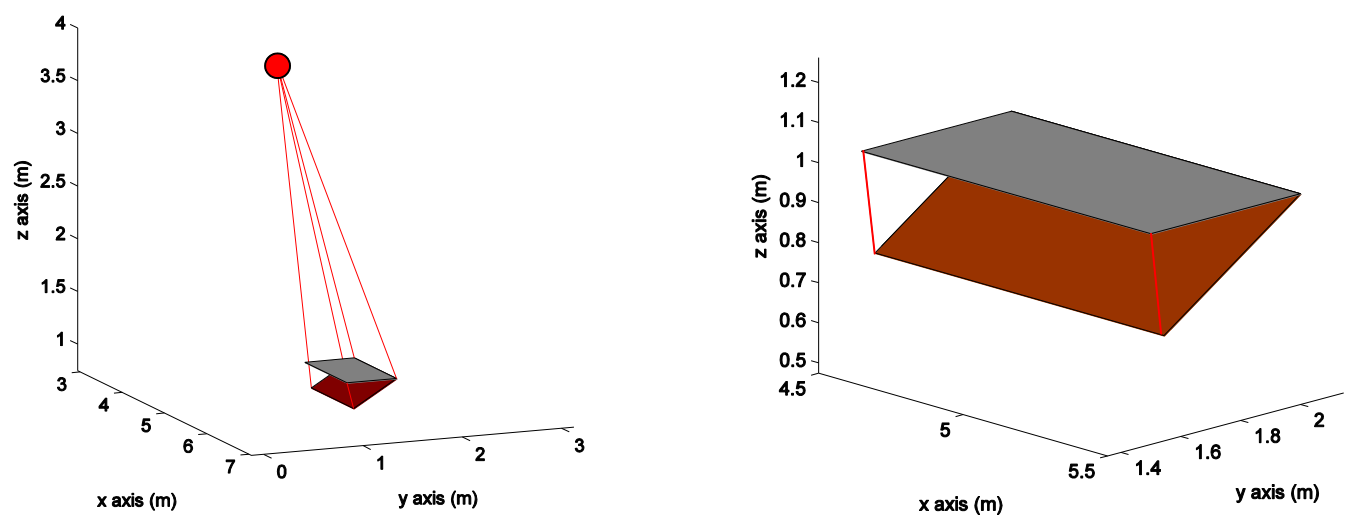

a)
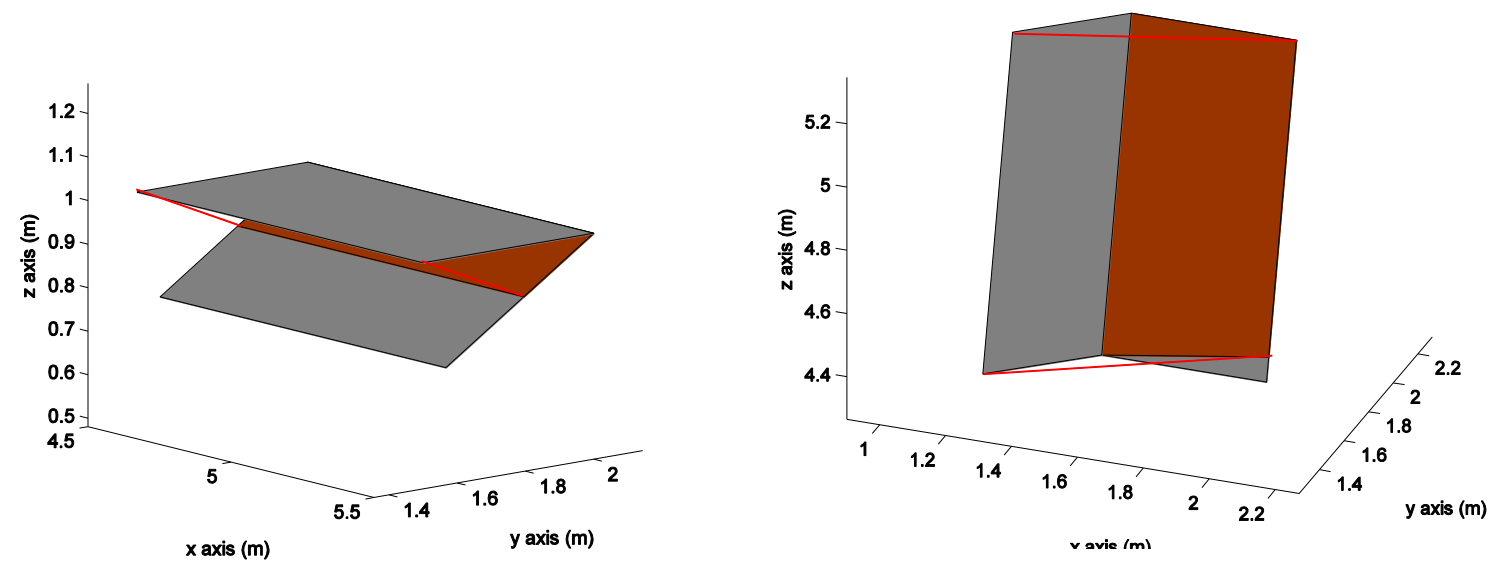

b)

c)

Fig. 47 Self-shadowing area simulation a) light source above the model and full shadow on the second plane b) shadow area after moving light source position c) shadow area after rotating. 


\subsection{Average solar radiation pressure for rigid debris}

The attitude of a piece of debris will alter the effects of solar radiation pressure through Eq. (1). For sake of comparison, it is beneficial to average the SRP force over the possible tumbling motion and use this averaged force to propagate over time in the case of the rigid debris. To reduce computational cost we decouple attitude and orbital dynamics. The results can then be averaged over one orbit to determine the averaged dynamics and compare it with the dynamics of the deformed model.

To model a tumbling piece of debris, it is assumed that it can adopt any orientation in the inertial space. Therefore, the expected value of the force is equal to the average force over all possible orientations. The average force is obtained by integrating over the solar latitude and longitude Eq. (1) and set $\hat{s}=[1,0,0]$.

$$
F_{\text {avg }}=\frac{1}{4 \pi} \int_{0}^{\pi} \int_{0}^{2 \pi} \vec{F}_{\text {rad }, j} d \lambda_{s} d \delta_{s}
$$

And the equivalent area is expressed as:

$$
A_{e q}=\frac{F_{a v g}}{P_{S P}(R)}
$$

Where $P_{S P}(R)=\frac{E}{C} \frac{A_{\oplus}^{2}}{\left|\overrightarrow{x_{i}}-\overrightarrow{x_{\oplus}}\right|^{2}}$ is solar pressure per unit area. Then, the average SRP can be calculated as:

$$
F_{A V G}=-A_{e q} P_{S P}(R) \frac{\overrightarrow{x_{i}}-\overrightarrow{x_{\oplus}}}{\| \overrightarrow{x_{i}-\overrightarrow{x_{\oplus}} \|}}
$$

\subsection{Simulation analysis}

Two different kinds of MLI [42] are selected for analysis: PET ${ }^{\circledR}$ and Kapton ${ }^{\circledR}$. PET $^{\circledast}$ is coated aluminium on both sides while Kapton ${ }^{\circledR}$ is coated only on one side. Their properties are listed in Table 5. The initial geometry is flat for both rigid and deformed debris. All objects start with the same set of Keplerian elements, which have a semi-major axis $41254.0 \mathrm{~km}$ eccentricity of 0.0001 , inclination of 5.0 degrees, argument of perigee of 9.0 degrees, argument of perigee of 9.0 degree, mean anomaly 270.0 degrees. Initial Euler angles of both planes are chosen from 0, 90 and 180 degrees (3-1-3 sequence) and they allow to orient the membrane at no particular direction. The initial angular velocity set zero around all directions.

The orbit and attitude evolution of both debris models has been investigated under various perturbations. J2 perturbation is labelled with subscript "J", Sun and moon third body gravitational perturbations are labelled with subscript "g", while the SRP force is labelled with subscript "s". Self-shadowing is denoted by subscript "h". The rigid body and flexible body have subscript " $\mathrm{r}$ " and subscript "f" respectively.

Table 5 Properties of PET and Kapton [42]. 


\begin{tabular}{llccc}
\hline Material type & $\begin{array}{c}\text { AMR } \\
{[\mathrm{m} 2 / \mathrm{kg}]}\end{array}$ & $\begin{array}{c}\text { Young's } \\
\text { Modulus } \\
{[\mathrm{N} / \mathrm{m} 2]}\end{array}$ & $\mathrm{Cs}, \mathrm{Cd}, \mathrm{Ca}$ \\
\hline PET & coated & 111.11 & $8.81 \times 10^{9}$ & 0.600 .260 .14 \\
\hline Kapton & coated & 26.30 & $2.50 \times 10^{9}$ & 0.600 .260 .14 \\
& $\begin{array}{l}\text { uncoate } \\
\text { d }\end{array}$ & 26.30 & & 0.000 .100 .90 \\
& & & \\
\hline
\end{tabular}

\subsubsection{Results and discussion}

Fig. 48 shows the dynamical evolution for the PET $^{\circledR}$ over 12 days under the Earth oblateness (J2) and solar radiation pressure. Both eccentricity and inclination evolutions of $f P E T_{j s, h}$ lay between $f P E T_{j s}$ and $r P E T_{j s .}$ Fig. 49 is analogous, but for Kapton.
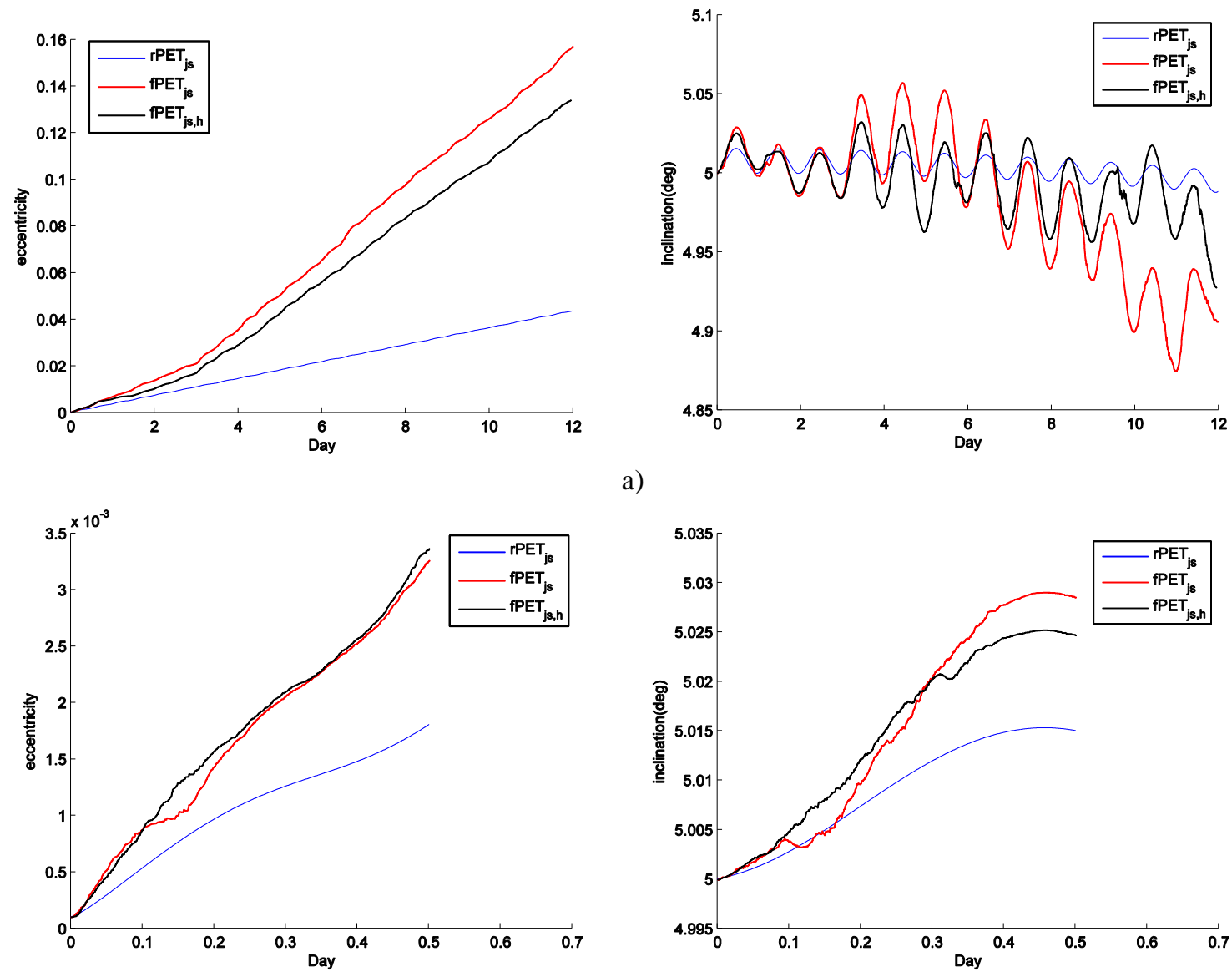

a)

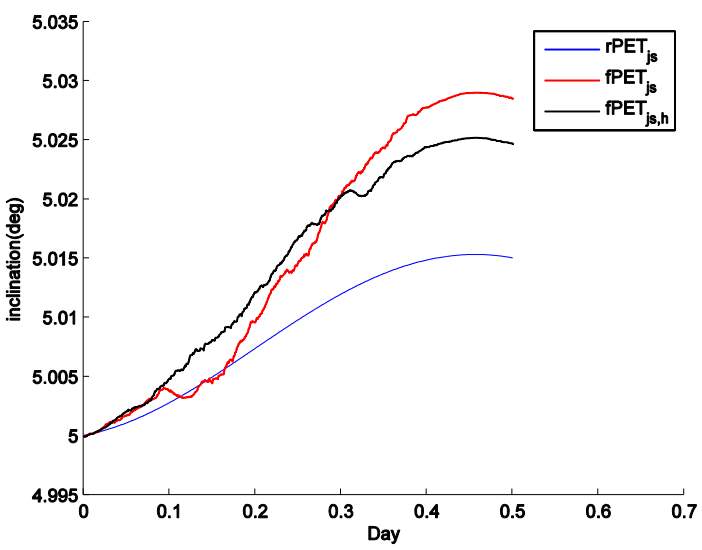

b)

Fig. 48 Comparison in eccentricity and inclination evolution of $f_{P E T}, f_{j s}{ } P T_{j s, h}$ and $r P E T_{j s}$ under $\mathrm{J} 2$ and solar radiation pressure a) evolution over 12 days b) evolution over 0.5 days 

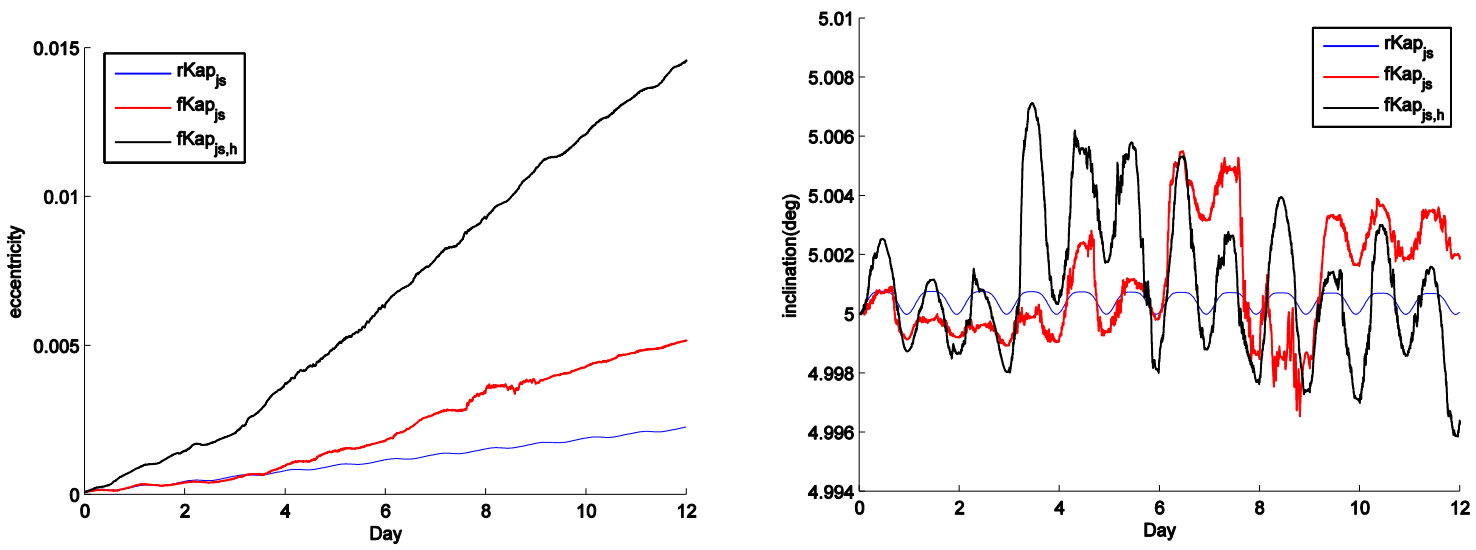

a)
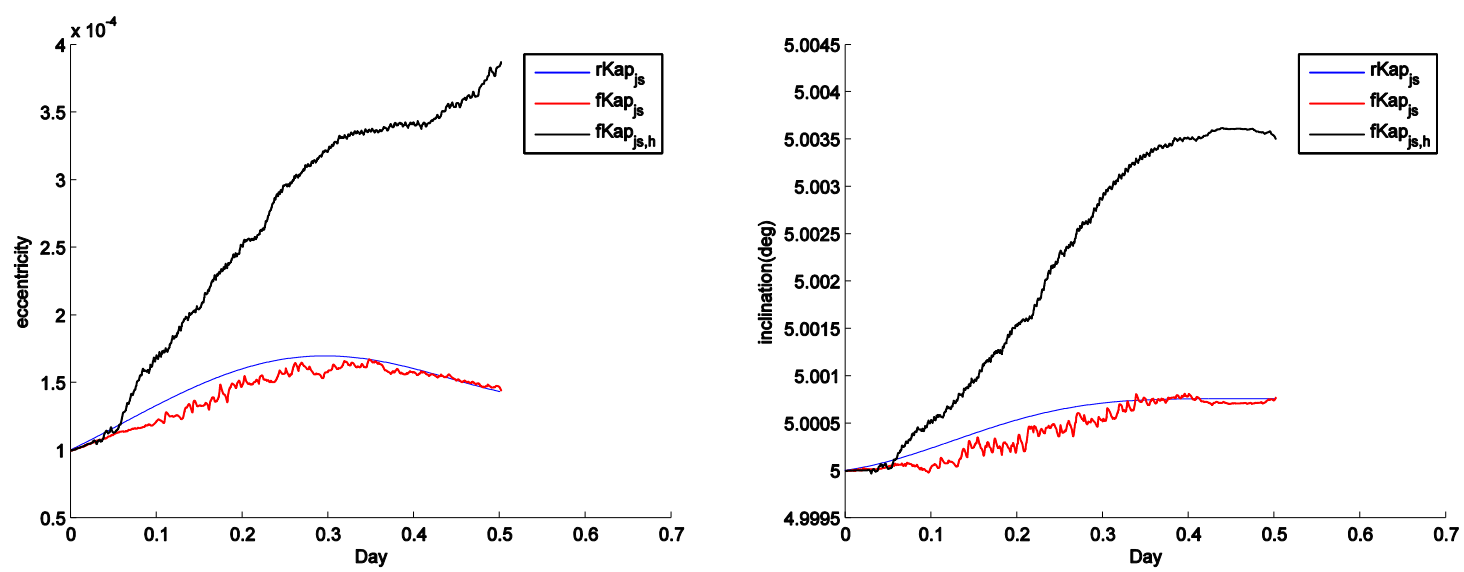

b)

Fig. 49. Comparison in eccentricity and inclination evolution of $f K a p_{j s}, f K a p_{j s, h}$ and $r K a p_{j s}$ under $\mathrm{J} 2$ and solar radiation pressure a) evolution over 12 days b) evolution over 0.5 day

To study the details of physical dynamics, we restrict the time of the investigation during 0.32-3.25 day (7.12 minutes) as in Fig. 50. The simulation shows a time-lapse of the membrane deformation and attitude state, absolute acceleration and Euler angles. It can recognize that deformed model is tumbling and changed their geometries during orbiting in Fig. 50(a). In this investigation, SRP is the dominating force to affect the dynamics. The sun vector can be assumed to be constant due to the investigation in a short period of time. The deformation and rotation are occurred when the solar radiation force is unbalanced on the two flat surfaces representing the membrane. Basically, an unbalanced SRP force on each flat surface depends on two main factors: first, the difference in incident angle on both planes due to the deformation and rotation of a flexible model. Second, the self-shadowing leading to the variations of exposed area.

To show the effect of different sun incidence angles between two planes, without considering the shadowing, $f P E T_{j s}$ has been simulated by neglected self-shadowing. Fig. 50(a) shows that an object can be deformed due to the difference incident angles. The absolute accelerations of both planes (Fig. 50(b)) are never zero due to SRP forces.

Conversely, Fig. 51(b) shows that the absolute acceleration in some periods gets to zero or very small, when selfshadowing is considered. Self-shadowing can occur in both planes during the simulation depending on which one is 
closer to the Sun as calculated by the self-shadow algorithm, and the shadow of a panel can cover some area of the one, or cover it completely. As the results of case without self-shadowing, $f P E T_{j s}$ is therefore exposed by higher the solar radiation pressure than $f P E T_{j s, h}$. This leads to the highest secular eccentricity gain and largest inclination variations than others in Fig. 48.

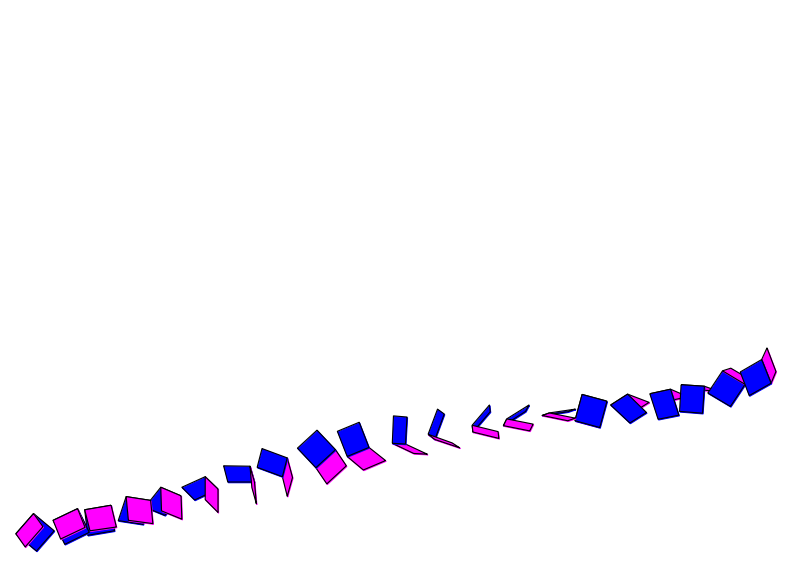

a)

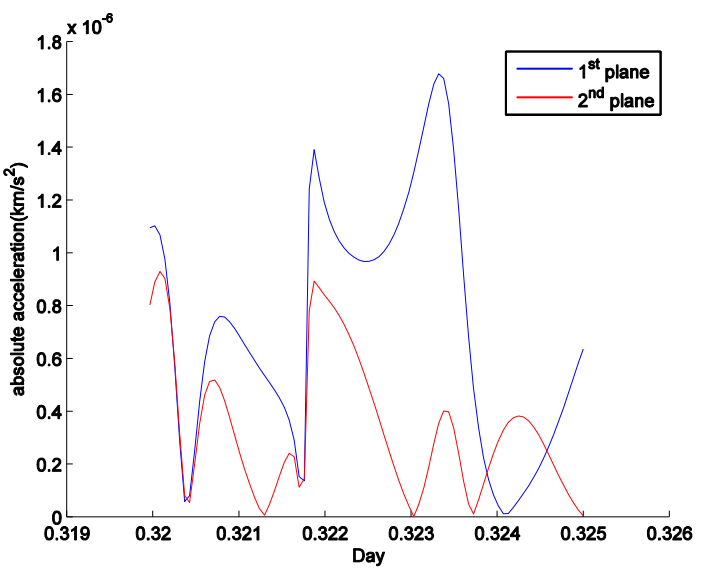

b)

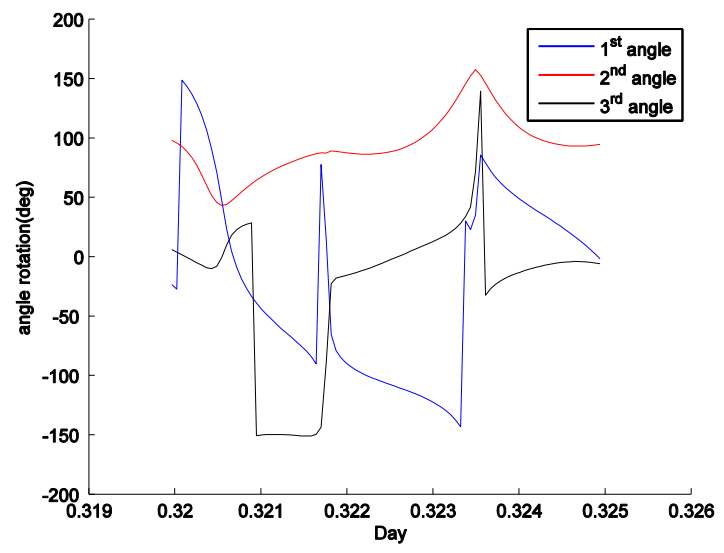

c)

Fig. 50 Simulation results of PET under $\mathrm{J} 2$ and solar radiation pressure without self-shadowing in 0.320-0.325 days (7.12 minutes) a) time-lapse of deformation b) absolute acceleration of both planes c) Euler angle evolution 


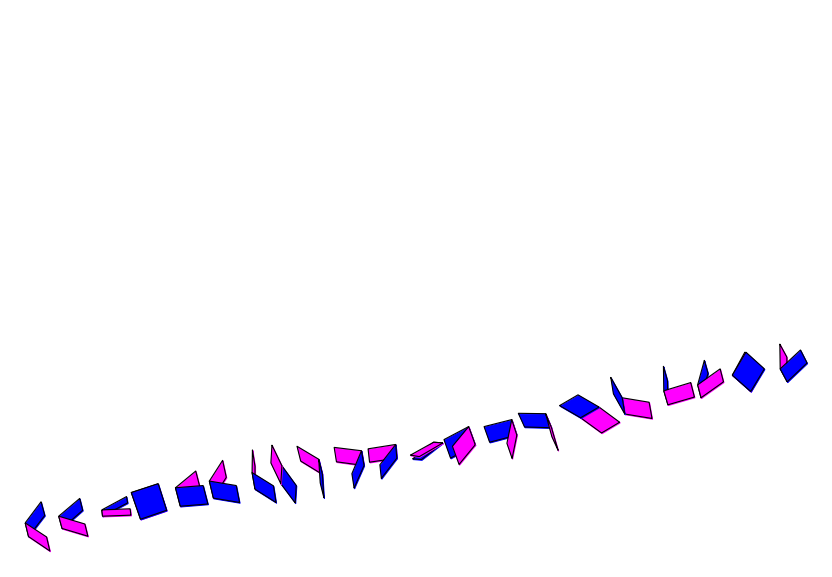

a)

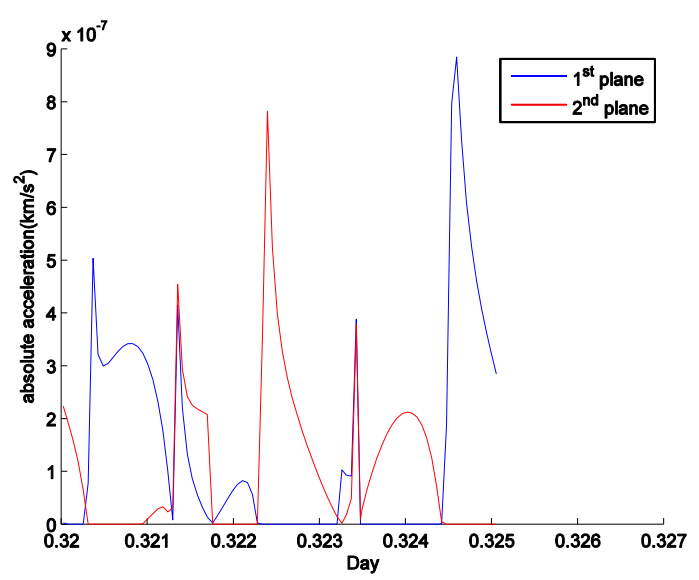

b)

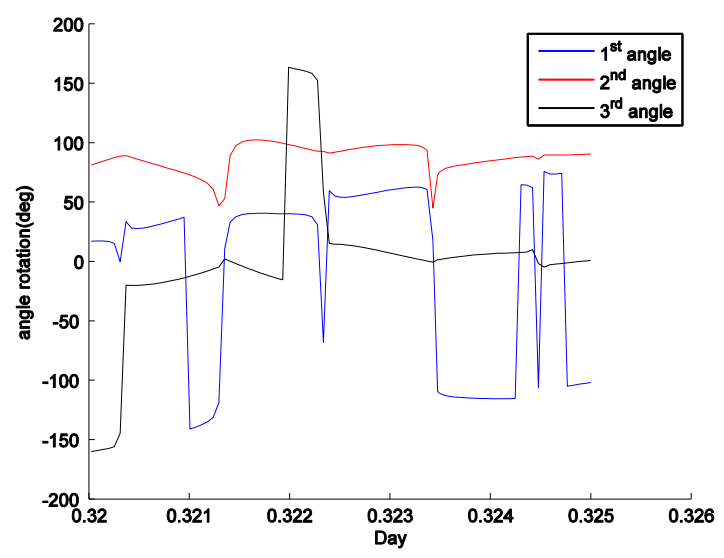

c)

Fig. 51 Simulation results of PET ${ }^{\circledR}$ under $\mathrm{J} 2$ and solar radiation pressure with self-shadowing in $0.320-0.325$ days (7.12 minutes) a) time-lapse of deformation b) absolute acceleration of both planes c) Euler angle evolution

Comparing the investigation of Kapton ${ }^{\circledR}$ under the same circumstances during 0.32-3.25 day (7.12 minutes), its reflection properties are non-uniform. The front surface is coated with aluminium that is highly reflective, while on the back side the reflectivity is very low. When we neglect self-shadowing, there are 2 phenomena explaining the dynamics. Firstly, if both reflective sides of the planes are exposed to SRP, the object will accelerate following the direction of the SRP forces (plus the gravity) if the difference incident angles of both planes is not large enough to deform it. If the SRP acts on both back sides, the object will essentially experience very little disturbance for orbt and attitude. Lastly, when the SRP acts on the front side of one plane and back side of the another one, it will activate deformation and tumbling. This leads the dynamics of $f K a p_{j s}$, shown in Fig. 52.

Comparing with $f \mathrm{Kap}_{j s, h}$, it is clear that the debris has higher potential to change a shape and rapidly rotate in Fig. 53(a) and Fig. 53(c) than for $f K_{a p}$, due to self-shadowing. The inclination and eccentricity evolutions in Fig. 49, therefore, illustrates that $f K a p_{j s, h}$ shows larger inclination variations and secular eccentricity variations than $f K a p_{j s}$, and $r K a p_{j s}$. 


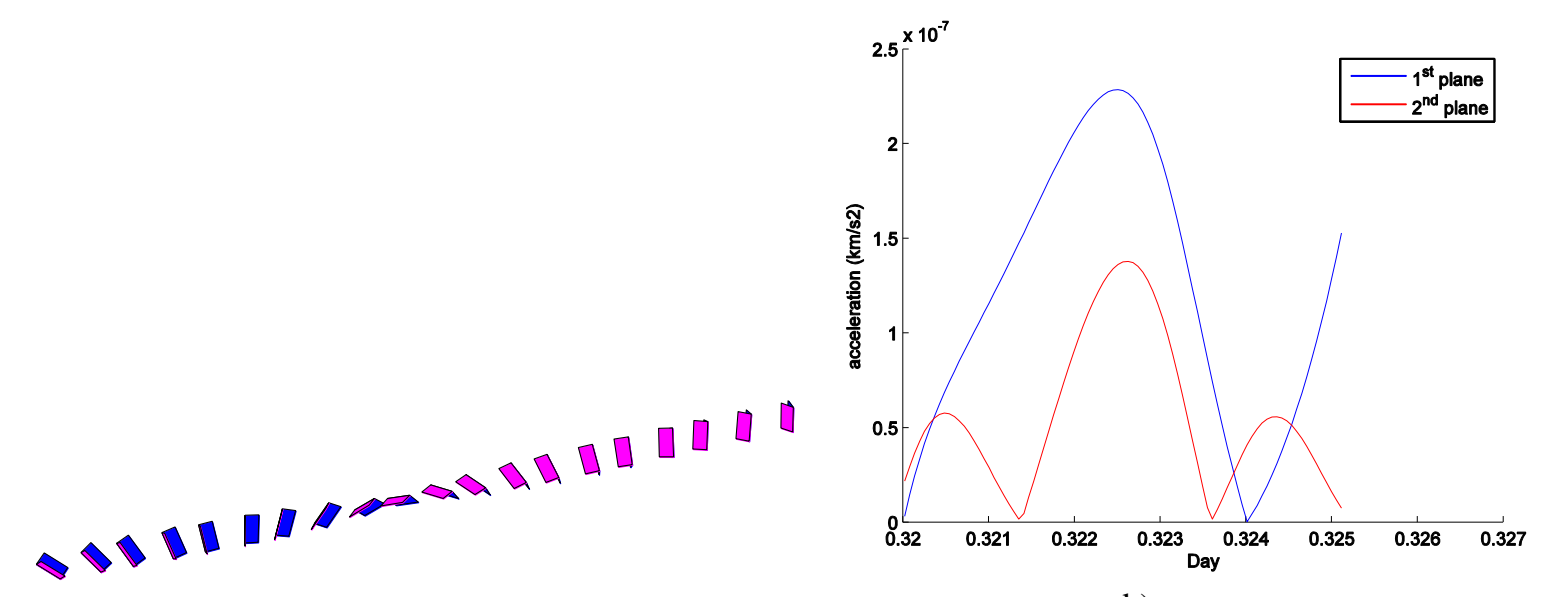

b)

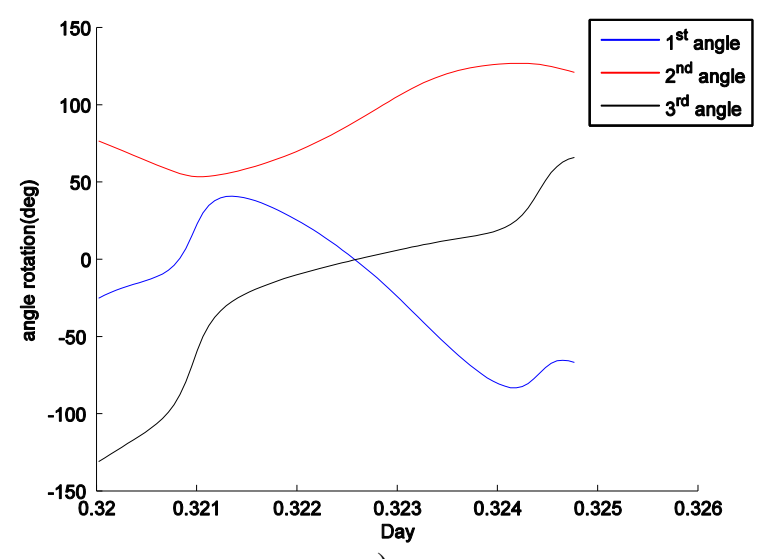

c)

Fig. 52. Simulation results of Kapton ${ }^{\circledR}$ under $\mathrm{J} 2$ and solar radiation pressure without self-shadowing in 0.3200.325 days (7.12 minutes) a) time-lapse of deformation b) absolute acceleration of both planes c) Euler angle evolution 


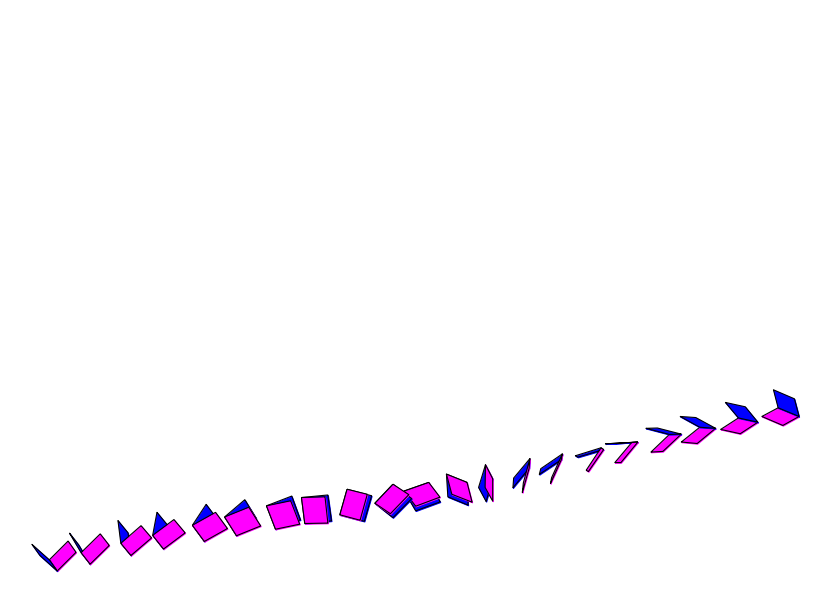

a)

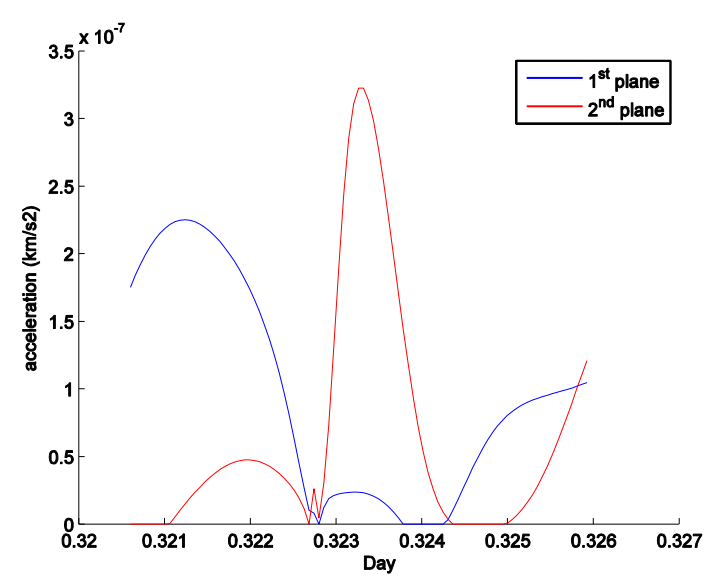

b)

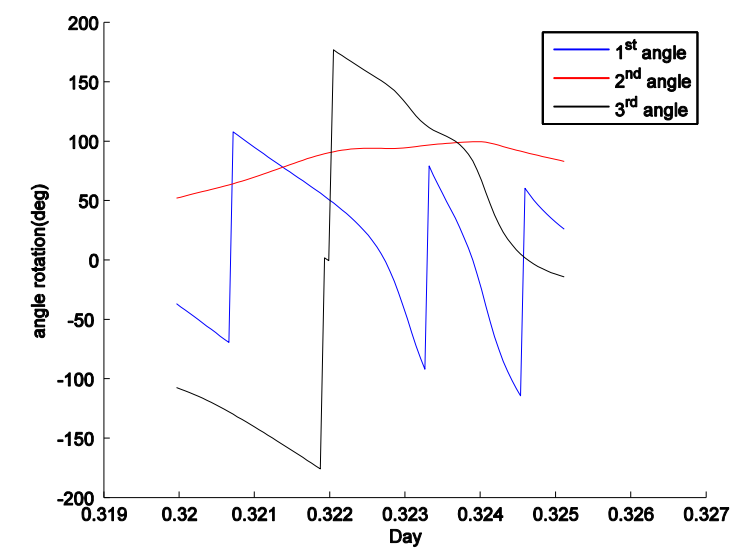

c)

Fig. 53 Simulation results of Kapton $^{\circledR}$ under $\mathrm{J} 2$ and solar radiation pressure with self-shadowing in 0.320 0.325 days (7.12 minutes) a) time-lapse of deformation b) absolute acceleration of both planes c) Euler angle evolution

The investigation of inclination and eccentricity under $\mathrm{J} 2$ and the third body perturbation in Fig. 54(b) illustrates that both evolutions of rigid and flexible models are not significantly different in orbital dynamics, as they differ in the order of $10^{-5}$ and $10^{-8}$ respectively. 


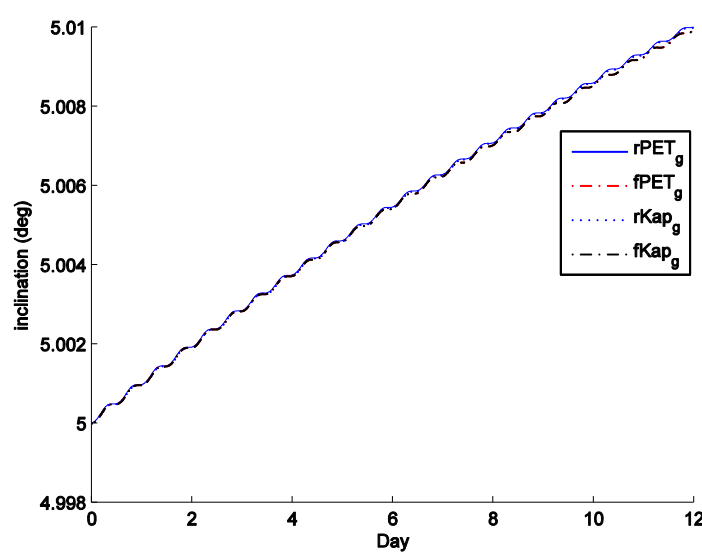

a)

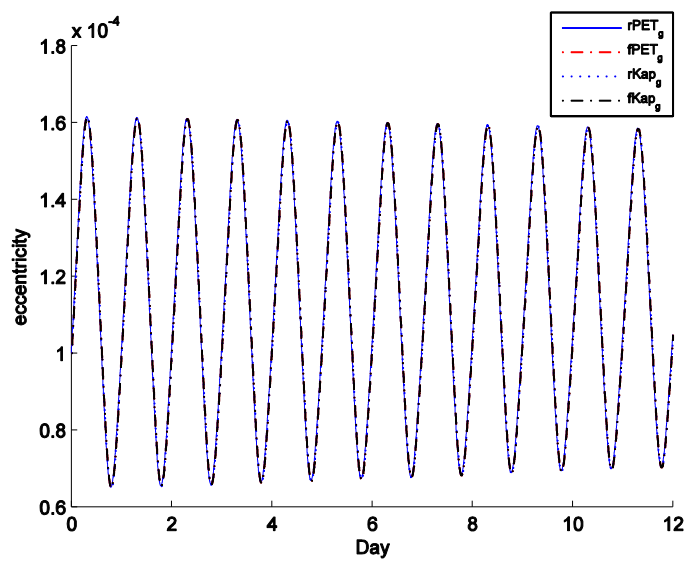

c)

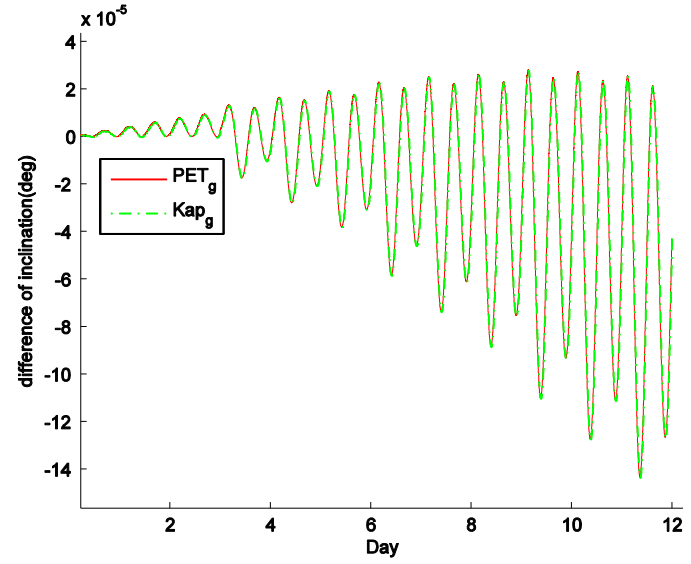

b)

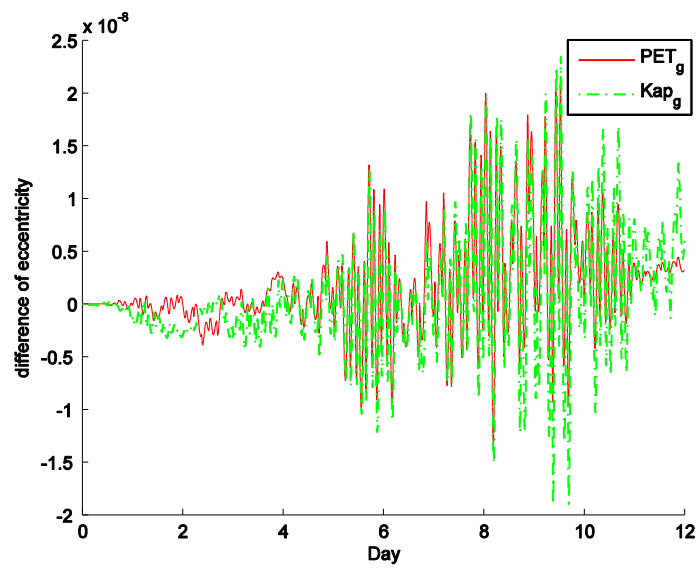

d)

Fig. 54 Comparison inclination and eccentricity evolution of $r P E T_{g}, f P E T_{g}, r K a p_{g}$ and fKap $_{g}$ under $\mathrm{J} 2$ and third body perturbations over 12 days a) Inclination evolution b) Difference in inclination between flexible model and rigid model. c) Eccentricity evolution d) Difference in eccentricity between flexible model and rigid

The next set of figures show the orbital evolution under all perturbations for the four object models ( $f P E T_{j g, h}$, $f K a p_{j g, h}, r P E T_{j g}$ and $r K a p_{j g}$ ) and will consider self-shadowing for the deformed model. Fig. 55 shows the trend in eccentricity and inclination. $f P E T_{j g, h}$ shows the highest amplitude of inclination oscillations and secular eccentricity gain. Considering the same material type in the flexible model, $f P E T$ has uniform reflection properties and larger AMR (because of its lower density) than that of Kapton ${ }^{\circledR}$ (around 4 times) leading to a greater inclination oscillation amplitude and a higher secular eccentricity increase. Moreover, it is evident from all simulations that variations of both inclination and eccentricity caused by the direct radiation pressure are dominating over gravitational perturbations.

Finally, for the self-shadowing and flexible model of both PET ${ }^{\circledR}$ and Kapton ${ }^{\circledR}$, we present for reference Fig. 56 and Fig. 57, which show their unique attitude dynamics over 1 day. 

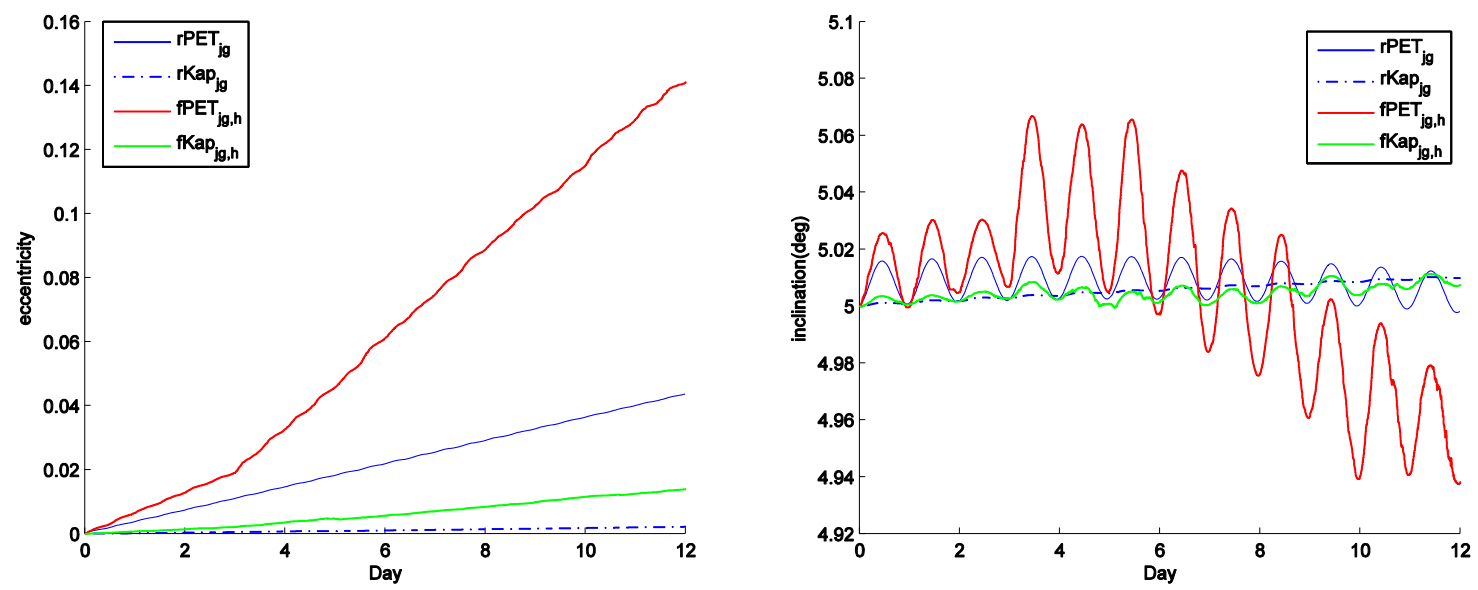

Fig. 55 Comparison in eccentricity and inclination evolution over 12 days of $f P E T_{j g, h}$, KKap $_{j g, h}, r P E T_{j g}$ and rKap $_{j g}$ under $\mathrm{J} 2$, third body perturbations and solar radiation pressure.

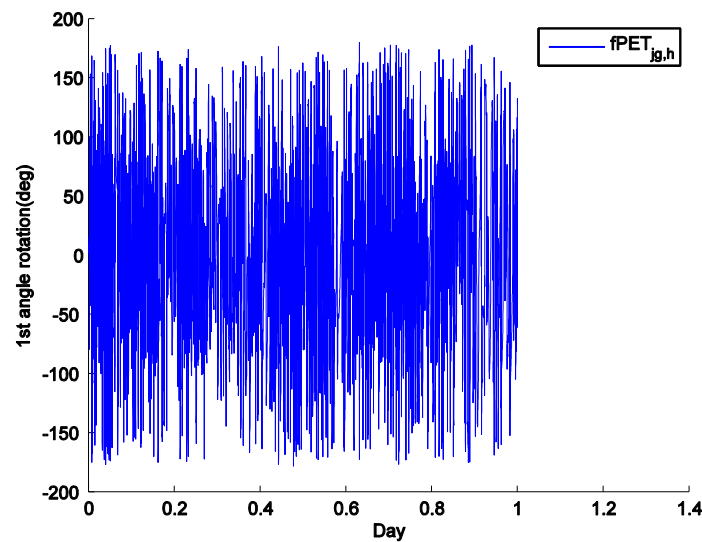

a)

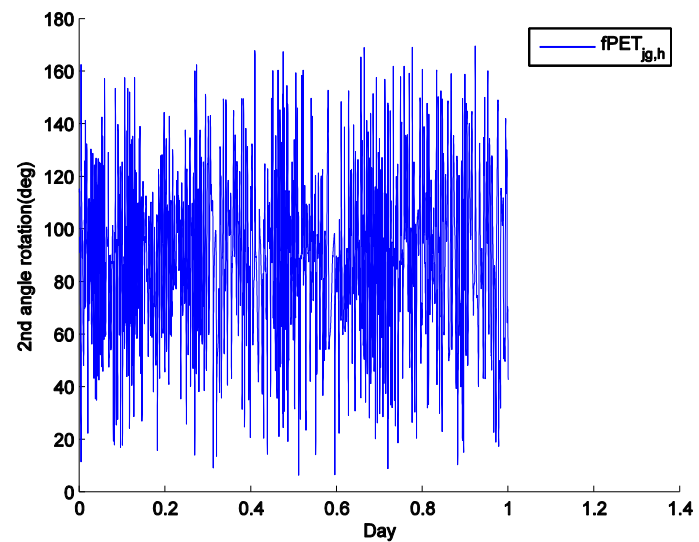

b)

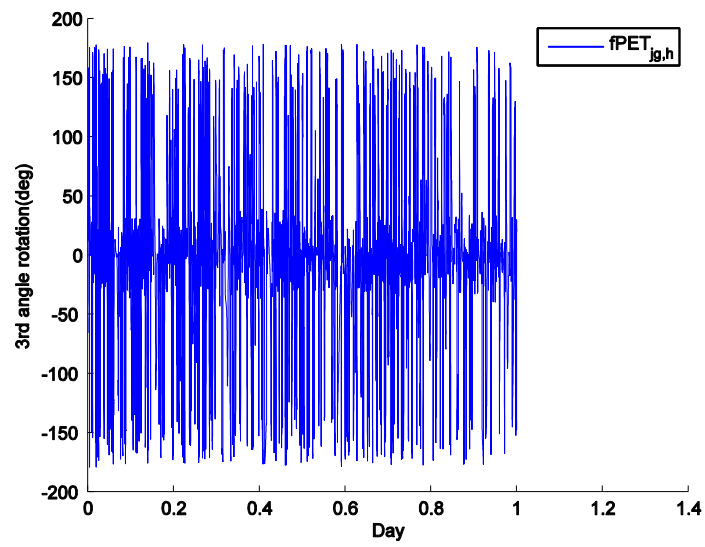

c)

Fig. 56 Euler angle evolution fPET , $_{\text {g,h }}$ over 1days under $\mathrm{J} 2$, third body perturbations and the direct solar radiation pressure a) $1^{\text {st }}$ Euler angle b) $2^{\text {nd }}$ Euler angle c) $3^{\text {rd }}$ Euler angle 


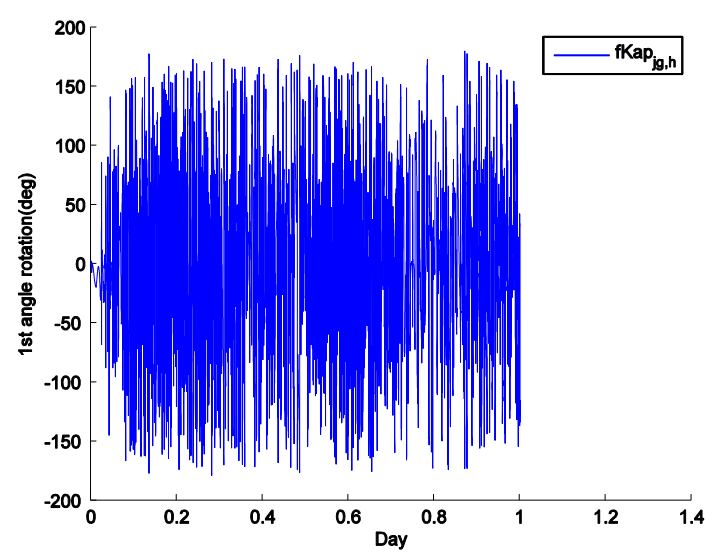

a)

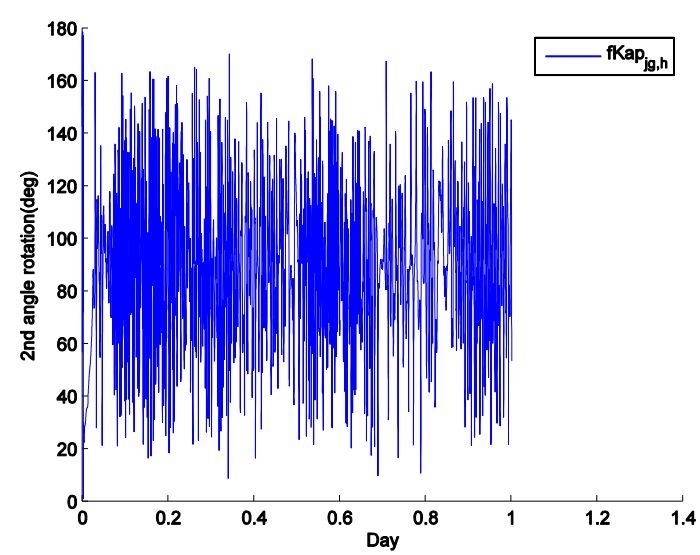

b)

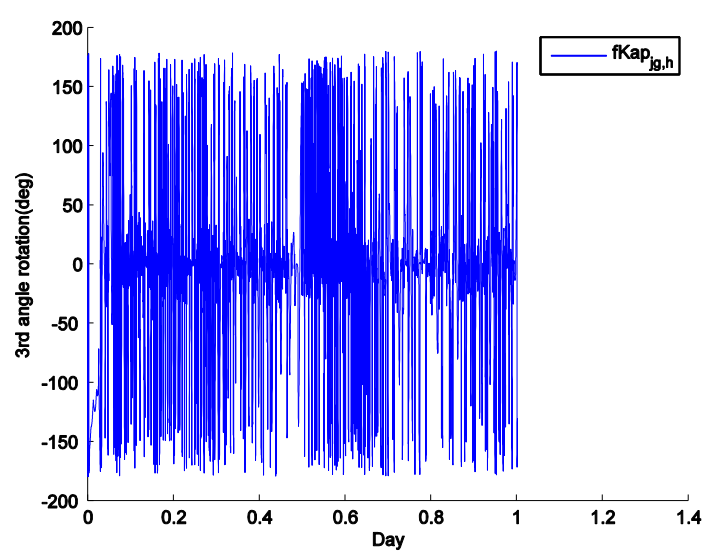

c)

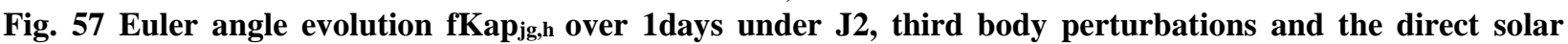
radiation pressure a) $1^{\text {st }}$ Euler angle b) $2^{\text {nd }}$ Euler angle c) $3^{\text {rd }}$ Euler angle

\subsection{Conclusion}

A new model for high area to mass ratio (HAMR) objects based on multi-body dynamics that is able to couple deformation, attitude and orbit dynamics in near GEO region has been presented. This model uses a full, non-linear set of equations for modelling the dynamics of a membrane, which is discretized with lump masses, a spring and a damper.

The results showed that perturbations from solar radiation pressure play a significant role in the dynamical evolution of the debris. The $\mathrm{J} 2$ and lunar-solar perturbations have a significant impact on the long-term orbit evolution. The large area-to-mass ratio is also the reason of oscillations in inclination and secular increase in eccentricity. The flexible model shows a complicate tumbling motion, which cannot be captured with rigid models. Also, the self-shadowing of the deformable model affects the AMR value leading to the unique rotational and orbital dynamics. A significantly larger secular trend occurs when ignoring self-shadowing in case of perfect reflection properties. The new model shows the significant different variations of inclination and high secular eccentricity including a rapid non-uniform attitude motion under an influence of direct solar radiation pressure and gravitation. 


\section{Propagation of coupled dynamics}

\subsection{Introduction}

In order to study the evolution of the orbit parameters of such objects over time, and improve the understanding of how does the perturbation affects the orbit parameters, a numeric integration of the orbit is commonly used [28, 29].

As discussed in the introduction, for long-term propagation, up to 50 years, the average of the AMR value was considered in [21]. The results were a common trend of 1 year period oscillation in eccentricity in GEO. Semianalytical propagation was applied and the value of AMR was reduced with defined coefficients in order to take into account tumbling, reflection and deformation effects. Short periodic effects were not considered and long periodic effects were integrated over one revolution. However, the reliability of the propagation over time does not seem to be accurate not only due to the average of the AMR value, but for possible resonances of the short period terms such as the harmonic of the Earth or the Sun-Earth-Moon configuration as it is studied in [26].

An average AMR value was also used in [28] to propagate four objects from the AIUB catalogue during 50 days. The ephemerides of the predicted orbit were compared with the observations of ZIMLAT, ESASDT and ISON network, and a significant difference in distance values was obtained. This study concludes that the values of AMR are function of time and do not show clear and obvious common trends. Therefore further study should be carried out in order to determine the cause of the change in AMR value.

The only non-average attitude study of MLI-Type debris found in the literature is carried out by Früh in [10]. The results lead to an overestimation of the AMR value if the orbital evolution is interpreted using the assumption of a stable attitude rather than a chaotic tumbling caused by SRP torque. The orbit propagation was carried out with a fully coupled orbit and attitude dynamics.

In all the debris simulations, the initial conditions for propagating the orbit are either obtained from light curves, orbit break-up simulations or fragmentation tests [43]. This allows more realistic initial attitude conditions and/or velocities.

In addition to the estimation of the AMR due to deformation, several studies showed that different modelling of eclipse leads to big errors when propagating the orbit $[44,45]$. This effect seems to greatly affect MLI-Type debris as stated in [29], when perfectly cylindrical shadow cone and umbra/penumbra model according to Baker were compared.

Clearly, the orbit prediction of HAMR debris objects, and in particular MLI-type debris, is a novel and challenging field of study where methods are being proposed and validated with observation data, with the aim of increasing the understanding of this new population of debris in order to consider mitigation procedures.

Observations from the ZIMLAT, ESASDT and ISON network from ESA showed an unstable value of AMR over time, which were attributed to possible attitude motion or deformation (see Fig. 58, from [28]). 


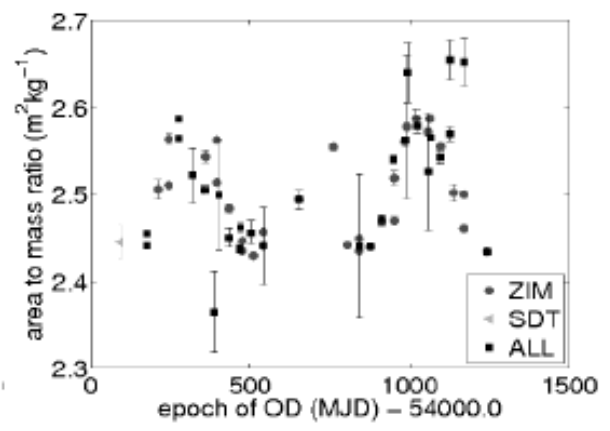

Fig. 58. Distribution of area to mass ratio (from [28]).

Although deformation has not been studied in the literature, fully coupled orbit and attitude motion of MLI debris was investigated in Früh [29]. Fast spin motion was found to be present in case of an offset between the center of pressure and the center of mass of the object, causing a high solar radiation torque. As it can be seen in Fig. 59 (taken from [29]), the frequency of spin is greater than the evolution of the orbit parameters in geosynchronous orbit.
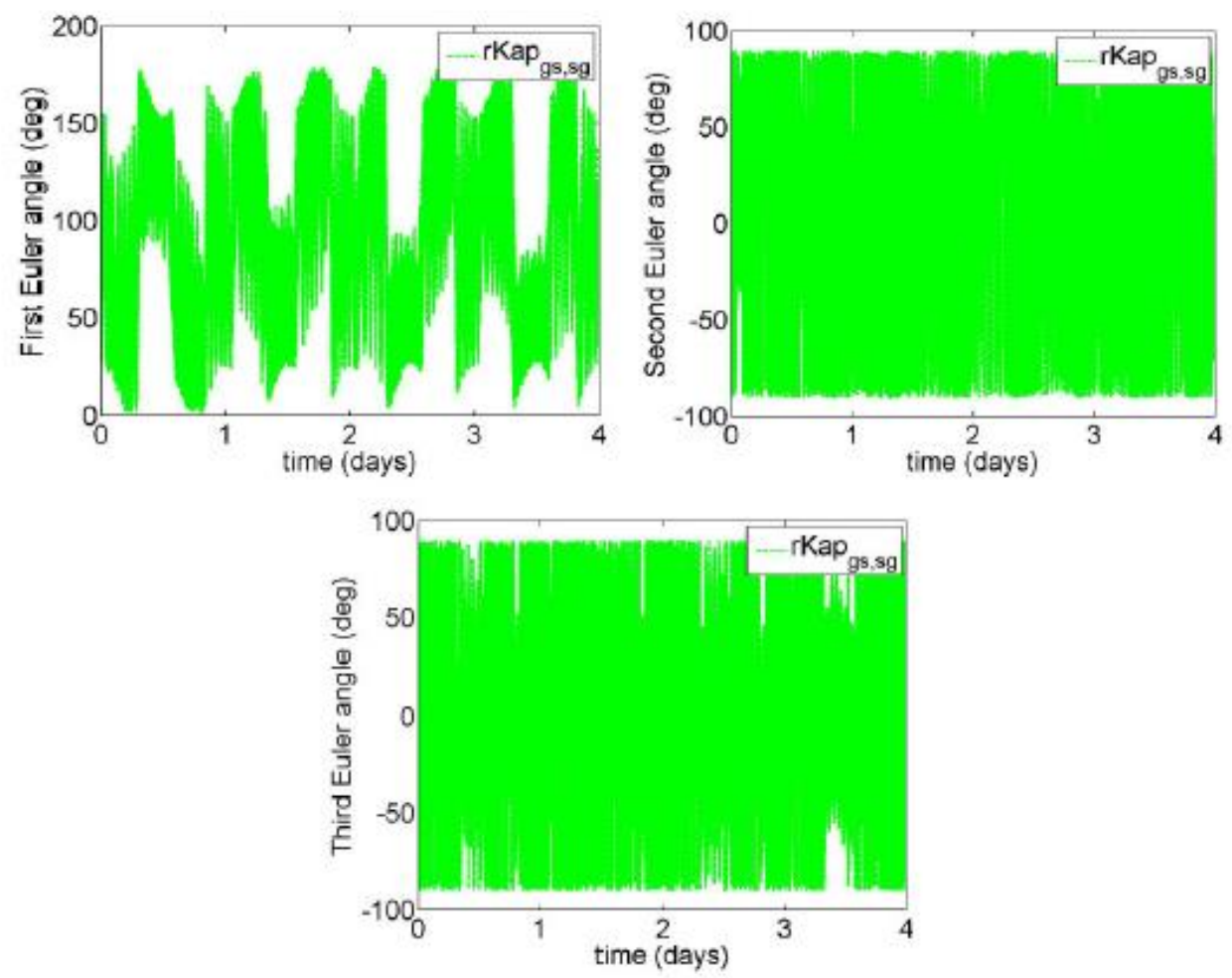

Fig. 59. Euler angles function of time (from [29]).

This offset may be caused by different reflectivity properties along the surface, for instance, due to the tendency of these objects to curl up during peeling off - observed in the degradation of the surface of the Hubble telescope -, or due to one end folded up due to deformation - this last one was the one considered in the simulation above. 
Therefore, a problem arise when simulating the coupled orbit and deformation of MLI debris with fast spin, as the frequencies of the spinning result in a severe increase of computational cost. Moreover, the nature of this type of debris requires dealing with non-negligible perturbations, and small integration steps for the equations of motion.

Fig. 60 shows the inter-dependence of the three dynamics for orbit, attitude and deformation. Each dynamics is essentially a set of differential equations, which are integrated starting from some given initial conditions. In principle, these dynamics are integrated separately, but their integration relies on parameters and states of the others, therefore cannot be simply decoupled. Assume the SRP as the only disturbance to the two-body orbital dynamics. The SRP force can be computed only when the following are known: the orbital position $\mathbf{r}$ (to give the relative position of the sun, eclipses); the attitude of the body with respect to the sun $\mathbf{q}$ and the state of deformation of the body $\mathbf{s}$ (defining the effective exposed area and direction of the force). The attitude states $\mathbf{q}$ and the deformation states s can be obtained integrating their respective dynamics. However the attitude dynamics require knowledge of the deformation state (for computing the inertia matrix) and the SRP torque; the deformation dynamics require knowledge of the SRP forces and torques acting on the body, as well as the angular velocity $\omega$.

It is also worth mentioning that in a diagram as in Fig. 60, the attitude dynamics assumes a rigid body, i.e. the inertia matrix of the body changes slowly with respect to the spinning rate, and therefore any change in the inertia matrix can be neglected in the Euler equations. This assumption might not be true for all types of deformable orbiting bodies.

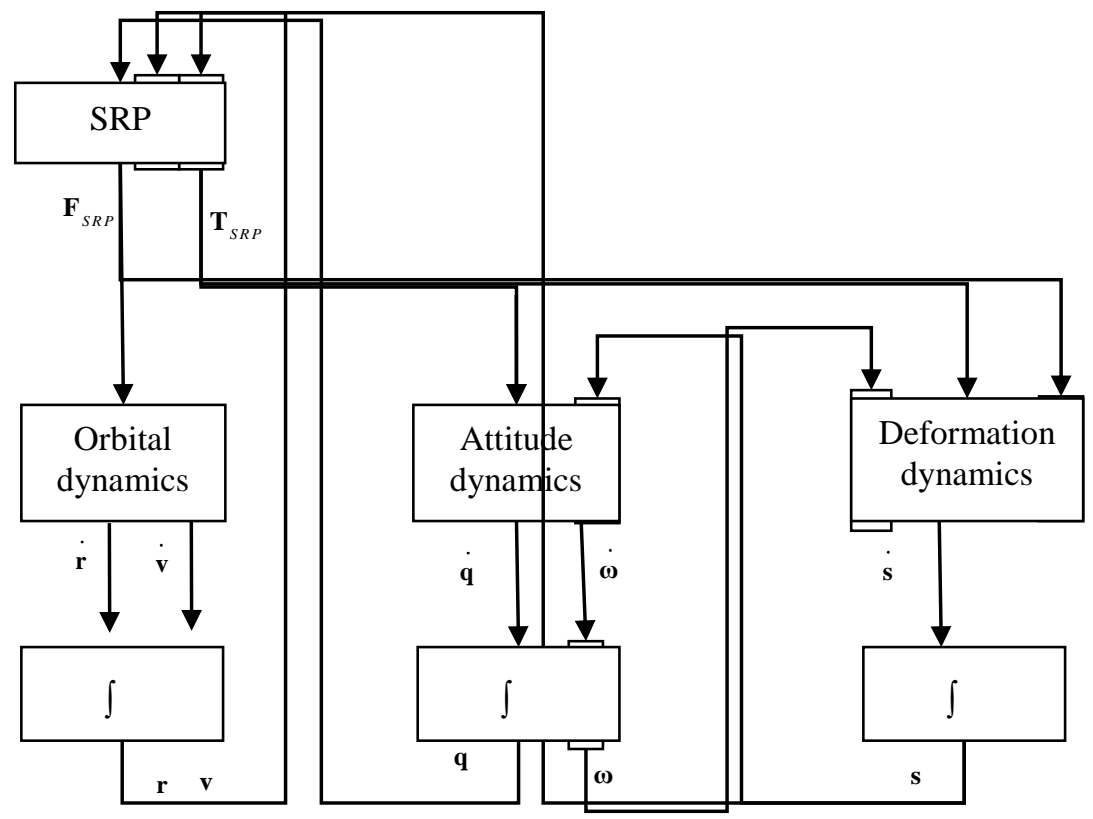

Fig. 60. Dependencies and interconnections among orbital, attitude and deformation dynamics. 


\subsection{Methodology}

The propagation of the orbit aims to calculate the evolution of the orbit over time, starting from some known initial conditions. The state vector of variables to be propagated over time can contain position and velocity (Cartesian elements), or the classical Keplerian parameters. The first method is known as Cowell's method, whilst the second method is referred as Variation of Parameters (VOP). The VOP method usually presents singularities depending upon the parameters selected and the orbit to be propagated. For this reason, other sets of elements were defined and used in the literature, such as equinoctial elements, which have their singularities in points of the parameter space that are generally not relevant to ordinary orbits).

When dealing with orbital perturbations, it is common to work with the Cartesian elements, since the perturbations, such as the Solar Radiation Pressure or drag, depend upon the position and/or velocity and cannot be directly computed using other orbital parameters, but instead the orbital parameters shall be converted into Cartesian elements beforehand, adding computational load on the algorithm at each time step.

The propagation of the orbit corresponds to a mathematic integration of the selected differential equations of the dynamics with respect to time, and thus three techniques can be applied: analytical, numerical and semi-analytical integration depending whether the integration can be solved directly or not.

Analytical methods show a clear advantage due to the high computational speed. However, the perturbations added to the main gravitational attraction must be modelled with a mathematical expression suitable for analytical integration. Generally, this mathematical modelling is obtained by expressing the perturbation with series expansions and truncating up to the desired order. The truncation usually causes a fast degradation of the results even with short period of propagation. Most analytical techniques make use of the variation of parameters proposed by Lagrange, which is only valid for conservative forces such as the harmonics of the gravity field.

Semi-analytical methods are partly analytical and partly numerical. They exploit the advantages of both methods, i.e. fast computation of analytic methods and accurate expressions for perturbation of numeric methods. The propagation of the orbit is split in two parts, an analytical integration for the two-body-problem, and a numeric integration for the perturbation dynamics. At pre-defined time steps, the two dynamics are coupled through a correction.

This method is efficient only if the perturbations are small when compared to the gravitational attraction, since this method relies on the fact that the perturbations are evaluated at a lower frequency, and assumed constant throughout a time interval. If this does not happen, then the correction should be carried out at a high frequency, and the advantage of this method is lost, and instead a fully coupled algorithm would be more efficient.

An example of a semi-analytical method is the Encke's method. Encke's method splits the acceleration experienced by the object in two contributions, one due to the exact two-body-problem dynamics and one considering all other perturbations:

$$
\ddot{\mathbf{r}}=-\frac{\mu}{r^{3}} \mathbf{r}+\mathbf{a}_{p}(\mathbf{r}, \dot{\mathbf{r}}, t, \ldots)
$$


If $\left|\mathbf{a}_{p}(\mathbf{r}, \dot{\mathbf{r}}, t, \ldots)\right|<<\left|-\frac{\mu}{r^{3}} \mathbf{r}\right|$, the two-body-problem can be integrated analytically, giving an accurate solution of the unperturbed (osculating) orbit within a small time interval, as seen in Fig. 61, where $\rho$ is the radius of the osculating orbit, $\mathbf{r}$ is the radius of the actual orbit, and $\delta \mathbf{r}$ is the difference in position between the two orbits, i.e. $\mathbf{r}=\boldsymbol{\rho}+\delta \mathbf{r}$.

\section{Fig. 61. Definition of position vectors.}

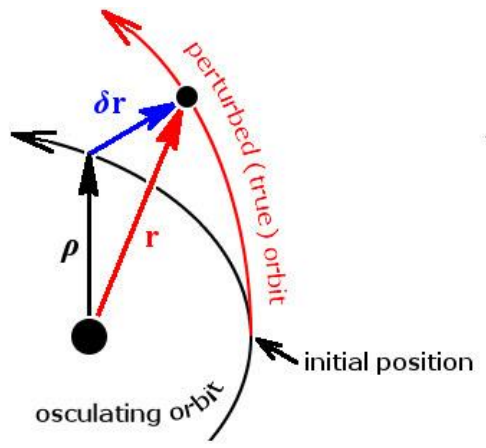

The osculating orbit is the exact solution of the two-body-problem for a given initial condition. It can be shown [32], that developing equation (3) leads to:

$$
\delta \ddot{\mathbf{r}}=-\frac{\mu}{\rho^{3}} \delta \mathbf{r}-\frac{\mu}{\rho^{3}}\left(\frac{\rho^{3}}{r}-1\right) \mathbf{r}+\mathbf{a}_{p}(\mathbf{r}, \dot{\mathbf{r}}, t, \ldots)
$$

The procedure is to firstly integrate analytically the two-body-problem to obtain the osculating orbit $\rho(t)$ with the initial state values. Then, Eq. (4) is integrated to obtain $\delta \mathbf{r}(t)$ until $|\delta \mathbf{r}|$ surpasses a certain tolerance. At this point, the algorithm is reinitialized again setting the new osculating orbit as the final real orbit obtained previously. The osculating orbit and the real orbit coincide at the time of correction, when $\delta \mathbf{r}=\delta \dot{\mathbf{r}}=0$. The tolerance can be set as:

$$
\frac{|\delta \mathbf{r}|}{|\mathbf{r}|}+\frac{|\delta \dot{\mathbf{r}}|}{|\dot{\mathbf{r}}|} \leq \varepsilon
$$

Numerical methods are widely applied to the propagation of the Cartesian state vector: Cowell's method and others. The main advantage of this method is that the perturbations are easily written as function of the state vector variables - position and velocity - and therefore they are directly added to the acceleration term and integrated.

Numerical integration methods can also be applied to the variation of parameters, when the perturbations are considered as non-conservative forces, making use of the Gauss equations.

An adaptation of the Encke's method has also been proposed [46] in order to add the known attitude dependence on the orbit dynamics (Fig. 62). The method consists in integrating both the osculating and the perturbed orbit through numeric integration. However, the osculating orbit may not be restricted to the two body problem, but can include orbit perturbation terms - such as higher harmonics of the gravity field - and it is integrated with a large time step, whereas the perturbed orbit takes into account the other perturbations related to the spacecraft attitude, and it is 
integrated with a small time step. As in Encke's method, a tolerance is introduced to reinitialize the algorithm to a new osculating orbit.

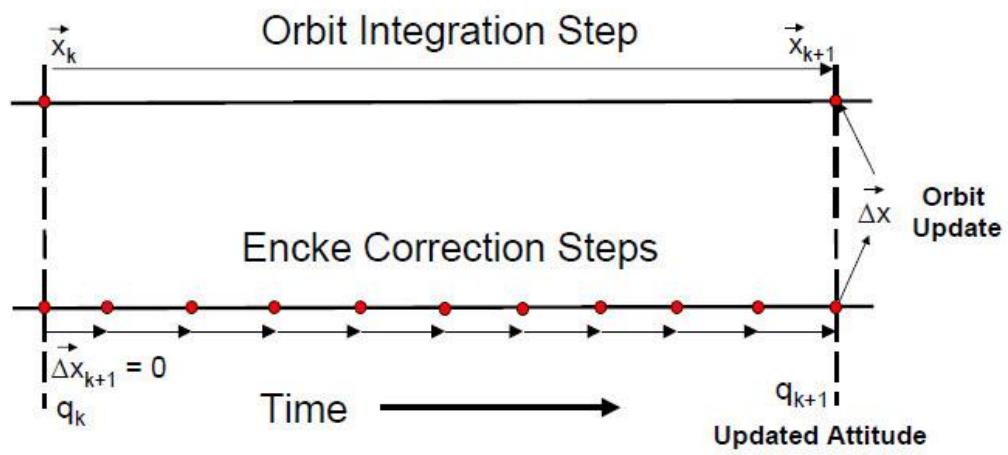

Fig. 62. Integration scheme.

\subsection{Numerical integration}

Numerical integration of the orbit is performed reducing the second-order differential equations of motion to a system of multiple first-order differential equations of the form $\dot{\mathbf{y}}=\mathbf{f}(\mathbf{y}, t)$, where $\mathbf{y}$ is the state vector. Given the initial conditions $\mathbf{y}_{0}$ at time $t=t_{0}$, the problem is known as initial value problem (IVP).

Numerical methods for first order differential equations discretizing the time-span and determining the value of the state vector $\mathbf{y}(t)$ at each time instant by computing the values of the derivative state vector $\dot{\mathbf{y}}$ at suitable timenodes: these can be previous points for explicit methods and following points for implicit methods. Depending on how many previous integration steps are used within the computation of the next step, the methods are classified as single-step methods or multi-step methods [47].

Multi-step methods use information from more than one previous integration step in order to predict and correct the values for the next step. Most of the multi-step methods are based on polynomial interpolation, and this is the main reason that is restricting theirs applications. Note that multi-step methods are not only restricted to explicit methods.

The most common single-step methods are the Runge-Kutta family. These methods are easily explained though the so-called Butcher tableau. Consider a Runge-Kutta method of $s$ number of stages, which means that within a time step, there are $s$ number of evaluations of the differential equations of motion. Define the node vector $\mathbf{a}[s \times 1]$ as the vector containing the times at which the dynamics is evaluated within each integration step. Now define the coupling matrix $\mathbf{b}\left[\begin{array}{lll}s & \mathrm{X} & s\end{array}\right]$ as the matrix used to compute the next state vector within the step as function of the previous or next derivative state vector. Finally, the weight vector $\mathbf{c}\left[\begin{array}{lll}1 & \mathrm{x} & s\end{array}\right]$ is the average weight for each evaluation. Using these vectors, the Butcher tableau is constructed as:

$$
\begin{array}{l|l}
\mathbf{a} & \mathbf{b} \\
\hline & \mathbf{c}
\end{array}
$$


For example, for the first-order Euler method the Butcher tableau is:

$$
\begin{array}{l|l}
0 & 0 \\
\hline & 1
\end{array}
$$

Higher order Runge-Kutta methods have been formulated, the most common the $4^{\text {th }}$ order "classic Runge-Kutta method" has the following Butcher tableau:

\begin{tabular}{c|rrrl}
0 & 0 & 0 & 0 & 0 \\
$1 / 2$ & $1 / 2$ & 0 & 0 & 0 \\
$1 / 2$ & 0 & $1 / 2$ & 0 & 0 \\
1 & 0 & 0 & 1 & 0 \\
\hline & $1 / 6$ & $1 / 3$ & $1 / 3$ & $1 / 6$
\end{tabular}

Note that for implicit methods matrix $\mathbf{b}$ may have nonzero values on the diagonal or on the upper triangle. To solve implicit methods, iterations must be carried out to convergence.

A really interesting family of Runge-Kutta methods is the embedded Runge-Kutta family. These methods, also called adaptive methods, estimate the truncation error by means of computing the difference of the values obtained from the $i^{\text {th }}$ and $(i+1)^{\text {th }}$ solution. Once the truncation error is estimated, the step size is adjusted according to a semiempirical formula. The same is used to decide whether the step shall be accepted or rejected and recomputed with a new (smaller) step size.

One of the most common adaptive methods is the Dormand-Prince 4(5), used in MATLAB's ode45 solver, with the following Butcher table:

Table 6. Dormand-Prince 4(5) Butcher table.

\begin{tabular}{c|ccccccc}
0 & 0 & 0 & 0 & 0 & 0 & 0 & 0 \\
$1 / 5$ & $1 / 5$ & 0 & 0 & 0 & 0 & 0 & 0 \\
$3 / 10$ & $3 / 40$ & $9 / 40$ & 0 & 0 & 0 & 0 & 0 \\
$4 / 5$ & $4 / 45$ & $-56 / 15$ & $32 / 9$ & 0 & 0 & 0 & 0 \\
$8 / 9$ & $19372 / 6561$ & $-25360 / 2187$ & $64448 / 6561$ & $-212 / 729$ & 0 & 0 & 0 \\
1 & $9017 / 3168$ & $-355 / 33$ & $46732 / 5247$ & $49 / 176$ & $-5103 / 18656$ & 0 & 0 \\
1 & $35 / 384$ & 0 & $500 / 1113$ & $125 / 192$ & $-2187 / 6784$ & $11 / 84$ & 0 \\
\hline & $5179 / 57600$ & 0 & $7571 / 16695$ & $393 / 640$ & $-92097 / 339200$ & $187 / 2100$ & $1 / 40$ \\
& $35 / 384$ & 0 & $500 / 1113$ & $125 / 192$ & $-2187 / 6784$ & $11 / 84$ & 0
\end{tabular}

Note that there are two vector c's: namely one is used in a $4^{\text {th }}$ order step $\left(\mathbf{c}_{4}\right)$ and one in a $5^{\text {th }}$ order step $\left(\mathbf{c}_{5}\right)$. The two steps are performed simultaneously, and their result is compared. This comparison provides an estimation of the truncation error $e_{T}$ performed by the algorithm, which is computed as follows:

$$
e_{T}=\left|h \mathbf{f}\left(\mathbf{c}_{4}-\mathbf{c}_{5}\right)\right|
$$

where $h$ is the (current) step size, $\mathbf{f}$ is the matrix containing the derivatives of the state vector evaluated at each time inside the step, at points specified by a. Finally, a correction for the step size can be computed as follows:

$$
h^{(i+1)}=z h^{(i)}\left(\frac{e_{T, \text { allowed }}}{e_{T}}\right)^{\frac{1}{p+1}}
$$


where $p$ is the order of the Runge-Kutta method, and $e_{T}$ is the allowed error for each step. In order to avoid too many rejected steps, an additional parameter $z$ is added. Common values around 0.85 are widely used [48]. Note that the previous expression is based on the fact that the local truncation error of the Runge-Kutta methods is one order higher than the order of the method.

Other algorithms for controlling the error of Runge-Kutta methods have been proposed, using two steps of the same order, but of different sizes, for estimating the truncation error. However, these methods have to compute a higher number of function evaluations per step, because the two step sizes do not share the evaluation of the dynamics at the same time. Instead, the embedded Runge-Kutta 4(5) method discussed above requires 5 evaluations for $\mathbf{c}_{5}$, out of which 4 are shared with $\mathbf{c}_{4}$, and therefore only 5 evaluations are necessary at each step.

\subsection{Algorithm}

An adaptive step, high-order Runge-Kutta-Fehlberg 7(8) integration method will be implemented and validated. This type of integration method has been already used in the literature [46] for integrating coupled orbit-attitude dynamics. An adaptive method such as the one considered is a good choice when dealing with unknown typical frequencies of the dynamics, and in particular the attitude one, since the step size is automatically readjusted for the given admitted relative and/or absolute errors. Also, in case of increasing or decreasing spinning rate of a debris object over time, the integrator will re-adapt the step size for optimal performance.

The algorithm has been adapted from [48]. The iterative integration procedure is shown in the data flow in Fig. 63. Essentially the differential equations of motion are evaluated at 13 different times within the given time step (according to the Runge-Kutta-Fehlberg 7(8) integration method), the truncation error $e_{T}$ is estimated and compared with the allowed error $e_{T, \text { allowed }}$. If the former is smaller than the latter, then the integration step is kept, and the time updated, else it is discarded. In both cases, the step size is updated based on $e_{T}$, and the procedure is repeated, until the final time is reached. 


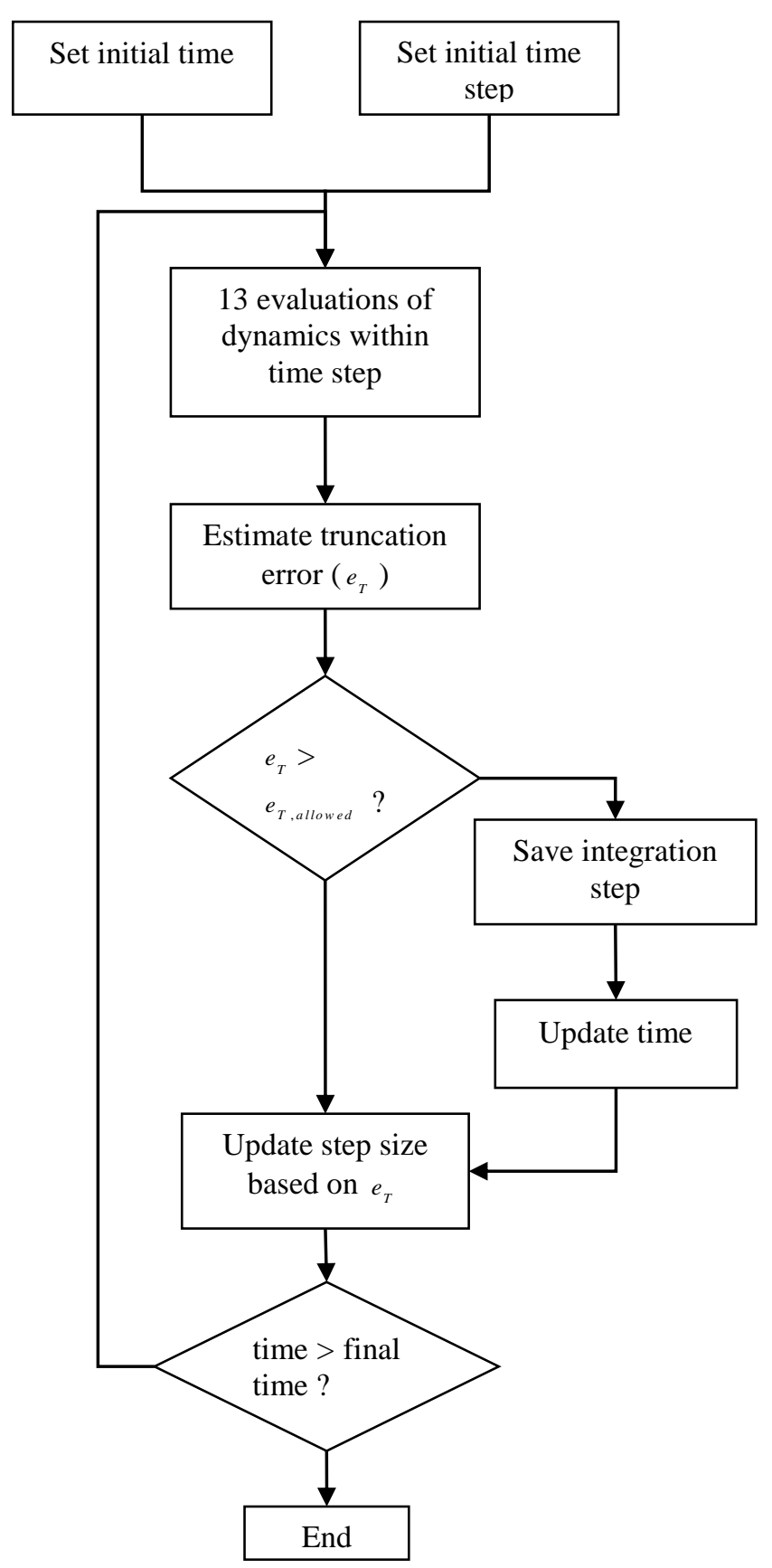

Fig. 63. Flow diagram for integration scheme.

The relative tolerance RelTol and absolute tolerance AbsTol are the parameters used to determine the accuracy of the integration, and they are used to compute the allowed truncation error $e_{T, \text { allowed }}$ at each integration step, as follows:

$$
e_{T, \text { allowed }}=\operatorname{RelTol}\left|y_{\max }\right|
$$


where $y_{\max }$ is the maximum value of the state vector. However, to avoid numerical problems with relatively small values of the state vector - for instance when determining the attitude in case the Euler angles are nearly zero - a minimum error will be enforced by using the absolute tolerance:

$$
e_{T, \text { allowed }}=\min \left(\operatorname{RelTol}\left|y_{\max }\right|, \text { AbsTol }\right)
$$

The step size update is obtained with the following equation:

$$
h^{(i+1)}=z h^{(i)}\left(\frac{e_{T, a l l o w e d}}{e_{T}+e p s}\right)^{1 / 8}
$$

The small number eps is added to the truncation error $e_{T}$ since this takes into account the round-off error of the calculator. The factor $z$ is introduced in order to tune the integrator and avoid unnecessary further rejected steps. Note that it must be $z<1$ such that the step size is reduced in case of a rejected step. It is set $z=0.85$.

In addition, a minimum step size is enforced in order to avoid step sizes becoming to small, which would lead to a sensible round-off error. The minimum step size is usually related with the current integration time:

$$
h_{\min }=16 \text { eps }(t)
$$

where the function eps $(t)$ is defined (in MATLAB) as the minimum value eps for which $t+e p s=t$.

The initial step size is calculated at the beginning of the integration for the first step, as follows:

$$
h^{(0)}=0.5\left(\text { RelTol }\left\|y_{0}\right\|\right)^{1 / 8}
$$

where the factor 0.5 again was added to avoid overestimation of the initial step size.

\subsection{Validation}

In order to test the implemented algorithm, the integration of a set of differential equations, for which an analytical solution is known, is considered. In this way, the numerical error of the integrator can be effectively measured. The error is defined as the maximum difference between the numeric solution and the analytic solution at any time step during the integration.

The proposed set of differential equations is the following:

$$
\dot{\mathbf{y}}=\left[\begin{array}{cc}
0 & 1 \\
-1 & 0
\end{array}\right] \mathbf{y}
$$

with initial condition $\mathbf{y}_{0}=\left[\begin{array}{ll}0 & 1\end{array}\right]^{T}$, for which the analytical solution is a unit-amplitude cosinusoid. This is integrated for 100 periods (final time $t_{f}=200 \pi$ ). The proposed integration method $(r k f 78)$ is compared in terms of error and function evaluation required against two other numerical methods: an own implementation of a Runge-KuttaFehlberg 4(5) integrator (rkf45) [49], and the MATLAB ode45. These results are presented in Table 7 to Table 10, for different values of AbsTol and RelTol. NFEVAL is the number of evaluations of the differential equations of dynamics, and we assume here that most of the algorithmic cost comes from evaluating these equations in a real 
case, and therefore the lower the number, the better the performance of the numerical integration, for the same accuracy. ERROR is, as mentioned before, the maximum absolute difference between the analytical and the numerical solution over the integration time span. NSTEP_ACC is the number of accepted integration steps, and NSTEP_REJ is the number of rejected one (i.e. step was recomputed with a smaller step size). Finally, as a reference, the computational time is given (in seconds). While this value depends on the machine used to run the algorithms, it is possible to compare the overall speed of the three approaches.

Table 7. AbsTol = RelTol $=$ 1.00E-6

\begin{tabular}{llll}
\hline & ode45 & rkf45 & $r k f 78$ \\
\hline NFEVAL & 19357 & 20406 & 7813 \\
NSTEP_ACC & 3226 & 3187 & 601 \\
NSTEP_REJ & 0 & 214 & 0 \\
ERROR & $5.92 E-05$ & $3.25 E-04$ & $4.50 \mathrm{E}-05$ \\
Time, $s$ & 1.67 & 1.37 & 0.452 \\
\hline
\end{tabular}

Table 8. AbsTol $=$ RelTol $=1.00 E-8$

\begin{tabular}{llll}
\hline & ode 45 & $r k f 45$ & $r k f 78$ \\
\hline NFEVAL & 49387 & 47748 & 14313 \\
NSTEP_ACC & 8231 & 7957 & 1101 \\
NSTEP_REJ & 0 & 1 & 0 \\
ERROR & $5.60 E-07$ & $3.64 \mathrm{E}-06$ & $1.62 \mathrm{E}-07$ \\
Time, $s$ & 3.98 & 3.46 & 0.796 \\
\hline
\end{tabular}

Table 9. AbsTol $=$ RelTol $=1.00 E-10$

\begin{tabular}{llll}
\hline & ode45 & rkf45 & $r k f 78$ \\
\hline NFEVAL & 124705 & 120150 & 25441 \\
NSTEP_ACC & 20784 & 20024 & 1957 \\
NSTEP_REJ & 0 & 1 & 0 \\
ERROR & $5.52 E-08$ & $3.60 \mathrm{E}-08$ & $8.59 \mathrm{E}-10$ \\
Time, $s$ & 8.97 & 10.963 & 1.48 \\
\hline
\end{tabular}

Table 10. AbsTol = RelTol = 1.00E-12

\begin{tabular}{llll}
\hline & ode 45 & $r k f 45$ & $r k f 78$ \\
\hline NFEVAL & 313831 & 301896 & 45279 \\
NSTEP_ACC & 52305 & 50315 & 3483 \\
NSTEP_REJ & 0 & 1 & 0 \\
ERROR & $5.50 E-11$ & $3.59 \mathrm{E}-10$ & $4.75 \mathrm{E}-12$ \\
Time, $s$ & 24.01 & 46.118 & 2.621 \\
\hline
\end{tabular}

It can be seen from the tables above that $r k f 78$ outperforms the other two 4(5) methods both in terms of function evaluations and error. The algorithm is considerably faster to run. ode 45 and $r k f 45$ are essentially equivalent in terms of number of function evaluations required, being based on a similar scheme, even if MATLAB's own implementation is optimized in terms of running time.

The conservative values used for $z$ and the coefficient 0.5 in Eq. (5) guaranteed that no step was rejected for any of the test cases in the $r k f 78$. 


\section{Orbit-attitude propagation techniques}

\subsection{Introduction}

In this chapter, we compare three different approaches for numerical propagation of coupled orbital and attitude dynamics of a body with finite dimensions (i.e. no point mass). Essentially the idea is to decouple the two dynamics, which might have different characteristic time scales, by integrating them separately. This allows integrating the slower dynamics with a longer time step, by achieving the same relative error of the faster dynamics, requiring a smaller time step. The integration with a longer time step will allow saving computational time. Of course, the two dynamics cannot be integrated completely independently, due to the fact that the states of one dynamics are parameters for the other one, and vice-versa.

Note that we are dealing with the dynamics of the orbit and the attitude, neglecting the dynamics of the deformation: we consider a rigid body. This simplifies the diagram in Fig. 60, by removing the deformation dynamics and all dependencies from $\mathbf{s}$. This is done for simplicity. Whilst the methods and results described in this chapter are applied to the coupled attitude/orbit problem, it is worth underlining that the methodologies proposed are quite general, and can be applied to any number of coupled dynamics, as long as they have different typical time scales.

In particular, the test case adopted is a rigid, high area-to-mass ratio MLI-type debris in near-geosynchronous orbit.

In the following, a study of three different approaches for the coupled attitude and orbit propagation problem will be studied and compared in terms of accuracy and computational efficiency.

The first approach is the integration of the fully coupled differential equations of motion for orbit and attitude. Essentially the two dynamics are always evaluated and integrated with the same time step, as if they were one. In this way, all the states are readily available at each time step, without any further calculation. This way, the time step of the integration will be determined by the fastest dynamics (namely the attitude, for a spinning body), and both dynamics will be solved at this time step. This formulation however, only allows propagation in short-term (few days), due to the computational cost required to keep the integration error low. Full coupling with short-term prediction has been studied by Früh [29].

The second approach decouples the attitude and orbit dynamics. The main advantage of decoupling the two dynamics is the possibility to evaluate the slower dynamics (the orbit in this case) with a large step size, therefore saving function evaluations and thus computational cost, as illustrated in Fig. 64. However, because one of the two dynamics is fast (namely the attitude), a small time-step size will still be necessary, and therefore it is predicted that even in this case, the propagation would still be limited to the short term. 


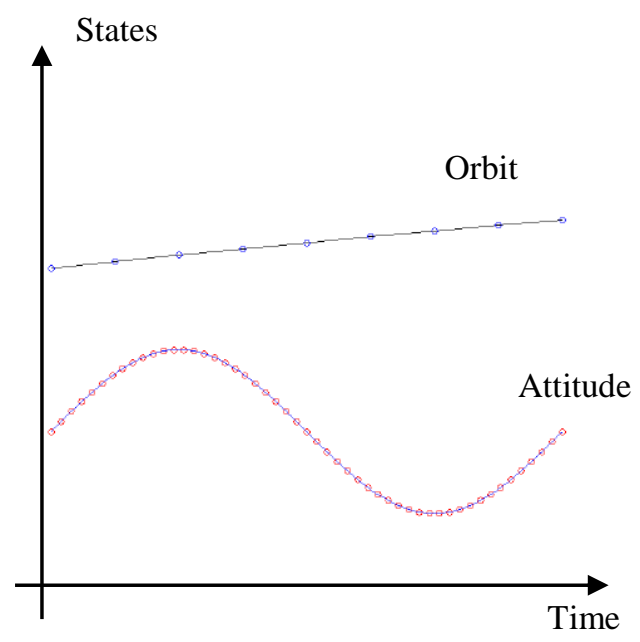

Fig. 64. Time histories of the dynamics of orbit and attitude.

The only solution for long-term propagation is to eliminate the fast dynamics of the problem. This allows using an overall larger step size, while maintaining the propagation accuracy. Fast dynamics can be eliminated with averaging techniques, when the fast motion is periodic. This is certainly the case for a spinning rigid body. Nevertheless, in this case, while the propagation itself can be very accurate, errors can still be present due to the averaging technique used. Averaging methods for long-term - up to 20 years - were used, for example, in [23].

It is worth mentioning that currently, techniques combining orbit prediction and observational data are being used for mid-term propagation $(\approx 50$ days). Essentially objects are tracked with a telescope to get an estimation of their position and velocity, when they are visible. These data are then used to propagate the orbit and predict the position of the next possible observation. This report however is focused on the propagation of the orbit only, assuming some initial data is available (e.g. from a previous observation) and assuming that no other observational data will be available during the propagation time (i.e. relying only on the initial conditions).

In the following section, a prototype of the problem is proposed, and the three different approaches (fullycoupled, decoupled and averaged) are studied in terms of accuracy.

\subsection{Formulation of the problem}

For an accurate propagation in geosynchronous orbit, the effects of third bodies (Moon and Sun), the harmonics of the Earth, and the perturbation of the SRP must be considered. For example, Früh [29], considered the harmonics of the Earth up to sixth order. In the same work, it is also found that, regarding the attitude motion, the SRP Torque is by means the most significant attitude perturbation, while the magnetic torque seems to be negligible. The gravity gradient can be significant depending on the cyclic differences of the principal moments of inertia of the body, which in turn depends on the body shape.

Because the aim of this chapter is to compare the different propagation techniques, as opposed to achieve a precise modelling of the dynamics and the perturbations, a prototype problem is created in the following way. First of all, the problem is planar. This allows reducing the number of states without changing the nature of the problem 
itself. The body is a rigid flat plate. The orbit dynamics is essentially the planar two-body problem, perturbed by the gravitational attraction of the Sun and the Moon (here fixed in an inertial Earth-centered frame), and the SRP force.

The attitude dynamics is based on the classic rotational equations of motion, and forced by the SRP torque.

The debris object is modeled as a rigid, square flat plate, of $l=1 \mathrm{~m}$ side, 20 microns thick, and the properties of the flat plate are as shown on Table 11. The area-to-mass ratio (AMR) (inverse of areal density) is that of PET, 111 $\mathrm{m}^{2} / \mathrm{kg}$. This allows to compute the total mass and the moment of inertia of the object.

In order to introduce torques due to SRP, we assume that the surface of the flat plate has two different parts, with different reflectivity properties (see Fig. 65). Most area of the debris is specularly reflective (coefficient of reflectivity 1), while one strip is fully absorbing the radiation (coefficient of reflectivity 0 ). Named $d$ the fraction of total surface that is non-reflective, the equivalent coefficient of reflectivity of the object is:

$$
c_{r}=1-d
$$

Different values of $\mathrm{d}$ will be used for the The solar radiation pressure force can be computed as:

$$
\mathbf{F}_{S R P}=P_{S R P}\left(1+c_{r}\right) \frac{A}{m} \cos ^{2}(\theta) \hat{\mathbf{n}}
$$

where $P_{S R P}=W_{0} / c$ with solar constant $W_{0}=1368 \mathrm{~W} / \mathrm{m}^{2}$ at Earth distance and $c=3 \times 10^{8} \mathrm{~m} / \mathrm{s}$ the speed of light in vacuum, $\theta$ is the angle between the unit normal to the surface $\hat{\mathbf{n}}$ and the Sun direction (see Fig. 66). The sign of $\mathbf{F}_{S R P}$ must be adjusted to make sure that it is always pointing in the semi-space away from the Sun.

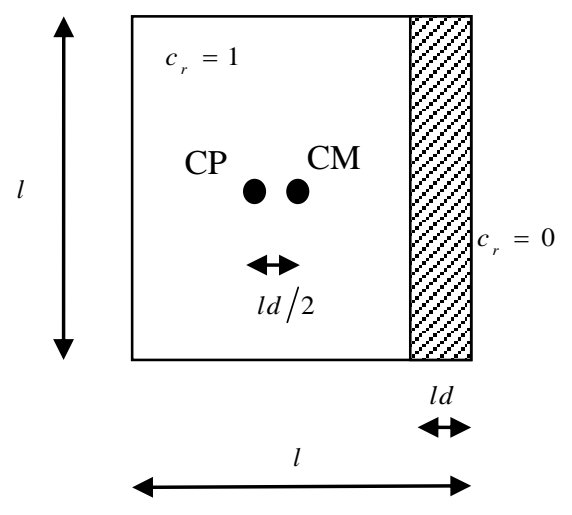

Fig. 65. Flat plate.

The effect of the non-reflective area is also to shift the center of pressure with respect to the center of mass (which is in the geometric center), by:

$$
r_{c m c p}=l d / 2
$$

This shift generates a solar radiation pressure torque $T_{S R P}=F_{S R P} l d / 2$. 
Table 11. Flat plate data.

\begin{tabular}{ll} 
Object side, $l$ & $1 \mathrm{~m}$ \\
Object thickness & $20 \mathrm{microns}$ \\
Area, $A$ & $1 \mathrm{~m}^{2}$ \\
AMR (area-to-mass ratio), $A / m$ & $111 \mathrm{~m}^{2} / \mathrm{kg}($ PET) \\
Mass & $9 \mathrm{~g}$ \\
Coefficient of reflectivity, reflective side & 1 \\
Coefficient of reflectivity, non-reflective side & 0 \\
\hline
\end{tabular}

The problem is then reduced to three degrees of freedom only: two translational and one rotational. The states to be integrated states include these three degrees of freedom, and their derivatives.
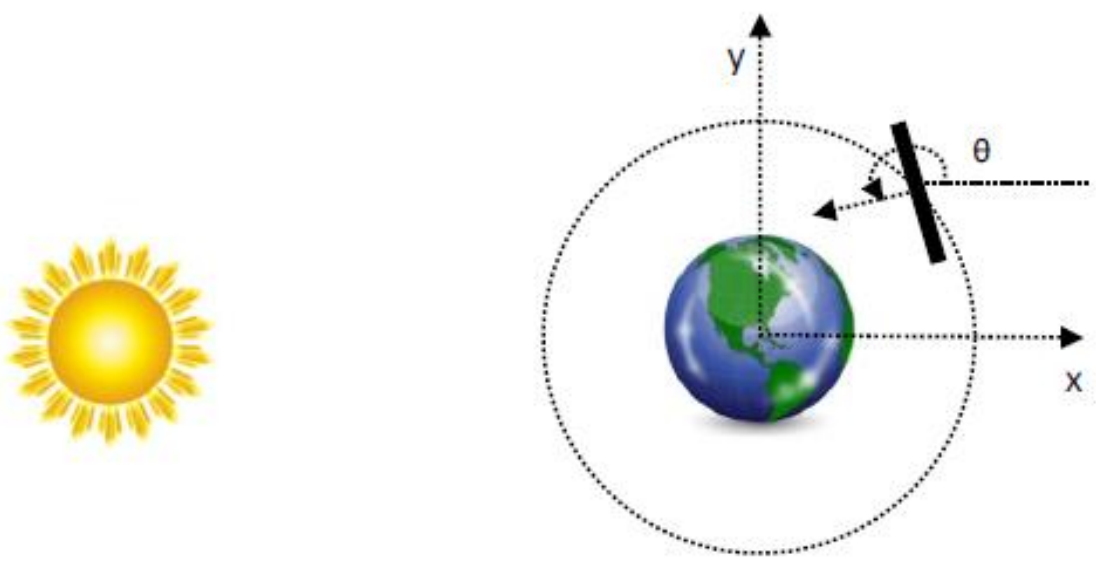

Fig. 66. Picture of orbital motion and definition of attitude angle $\theta$.

Naming the ECI orbital position vector $\mathbf{r}$, and the attitude parameter $\theta$, we have that the net acceleration on the body is:

$$
\mathbf{a}=\mathbf{a}_{E}(\mathbf{r})+\mathbf{a}_{S}(\mathbf{r})+\mathbf{a}_{M}(\mathbf{r})+\mathbf{a}_{S R P}(\mathbf{r}, \theta)
$$

This equation highlights the dependence of the acceleration due to Earth, Sun and Moon on the orbital position $\mathbf{r}$, while the SRP acceleration is responsible for the coupling of the dynamics, in that it depends on both $\mathbf{r}$ and attitude $\theta$. Similarly, the total torque acting on the body is:

$$
\mathbf{M}=\mathbf{M}_{S R P}(\mathbf{r}, \theta)+\mathbf{M}_{E}(\mathbf{r}, \theta)
$$

which highlights that both terms - gravity gradient torque and SRP torque - depend on both orbital position and attitude. Both attitude and orbital dynamics are inter-dependent upon each other, which is representative of a general case.

The integration is performed in an Earth-centered inertial (ECI) reference frame, with Cartesian coordinates, where the $x-y$ plane is arbitrarily the equatorial plane.

The initial orbit is geosynchronous, with initial state: 


$$
\begin{gathered}
\mathbf{r}_{0}=\left\{\begin{array}{c}
42241.08007 \\
0 \\
0
\end{array}\right] \mathrm{km} ; \quad \mathbf{v}_{0}=\left\{\begin{array}{c}
0 \\
3.071858028 \\
0
\end{array}\right] \mathrm{km} / \mathrm{s} \\
\theta_{0}=\dot{\theta}_{0}=0
\end{gathered}
$$

and the equations are integrated for $24 \mathrm{~h}=86400 \mathrm{~s}$.

Due to the integration lasting 1 day only, the position of the Sun and the Moon are fixed in an Earth-centered inertial frame. This assumption would not be valid for longer integration times, but it does not affect the results presented here nonetheless. The position of the Sun and the Moon (of gravitational constants $1.32712428 \mathrm{e} 11$ $\mathrm{km}^{\wedge} 3 / \mathrm{s}^{\wedge} 2$ and $\left.4902.799 \mathrm{~km}^{\wedge} 3 / \mathrm{s}^{\wedge} 2\right)$ in ECI are respectively:

$$
\mathbf{r}_{S}=\left\{\begin{array}{c}
-149,597,870 \\
0 \\
0
\end{array}\right] k m ; \quad \mathbf{r}_{M}=\left\{\begin{array}{c}
384,399 \\
0 \\
0
\end{array}\right] k m
$$

\subsection{Description of the three approaches}

\subsubsection{Fully-coupled approach}

Fully-coupled dynamics is the conventional way to integrate different dynamics in case they depend each other. However, in case of really fast spinning of MLI-Type debris, this approach is limited to short prediction times for two reasons: firstly, the computational cost is high, and secondly, the integrator error increases quickly. Fig. 67 is a diagram showing the data flow of a fully-coupled dynamics simulation.

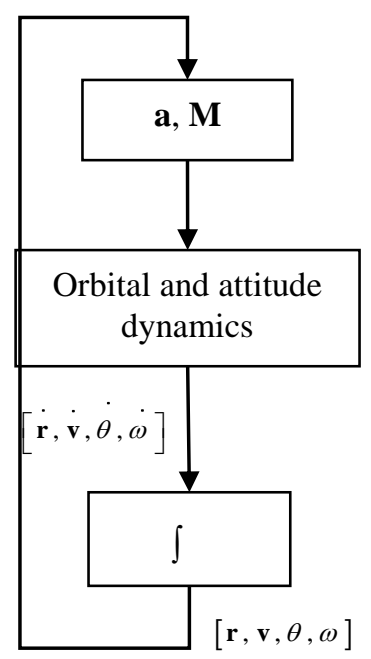

Fig. 67. Flow diagram of fully-coupled orbital and attitude dynamics. 
In addition, due to the particular configuration of the flat plate chosen for this simulation, the spin rate increases continuously due to the SRP torque, and this causes the step size to reduce along the simulation. Hence, because of this increasing angular velocity, the process of averaging the attitude motion must be studied in detail in order to predict long-term behavior of this kind of debris.

\subsubsection{Decoupled approach}

Decoupled approaches for integrating dynamics with different timescales were found in the literature of electric circuit simulation (e.g. [50]) for decoupling electric signals with different time scales. Essentially, the approach is to use multi-rate Runge-Kutta methods. These integrators are fixed-step methods, and the coupling of the signals is made by interpolating or extrapolating the signal which is not being propagated for a given time. In a similar way, a decoupled Runge-Kutta method is proposed and studied in terms of accuracy, and compared to the fully-coupled approach, which uses a conventional Runge-Kutta scheme.

As stated before, the core of this formulation is in that it allows evaluating the (fast) attitude dynamics with a small time step, and at the same time skipping some evaluations of the orbital dynamics differential equations, saving computational cost.

\subsubsection{Formulation}

We make use of the Encke's method of propagation of the osculating orbit, and in first instance consider that over one time step of the orbital dynamics $\Delta H$, the two-body problem is a good approximation, and neglect the perturbations and the effect of the change in attitude during this time. This implies that the perturbations over the classical two-body problem dynamics are very small compared to the gravitational acceleration. The time step $\Delta H$ is several times greater than that of the attitude dynamics, which is integrated with step $\Delta h$. The orbit estimation allows calculating the evolution of position and velocity after time $\Delta H$, and at each instant of time within $\Delta H$ through interpolation. The idea is that, despite this orbital prediction is erroneous, due to the fact that any variation is attitude (and therefore variation in SRP) is not taken into account, on the other hand these variations would produce a negligible impact on the single step. In particular, the impact is negligible with respect to the attitude calculation, in that the torques do not change dramatically for minor variations in the orbital state.

This first estimation of the orbital state is used to propagate the attitude dynamics along the orbital time step $(\Delta H)$, this time using a number of smaller steps $\Delta h$ (refer again to Fig. 64). Despite the attitude propagation uses a slightly erroneous orbital prediction, as discussed before it is assumed that this error is negligible, and therefore the attitude history obtained at this step is final.

Finally, the attitude time history over $\Delta H$ is interpolated and used to correct the position and velocity prediction obtained before.

The entire procedure is started with the orbital and attitude initial conditions, and iterates for each step $\Delta H$. One of these steps is represented as a flow diagram in Fig. 68. Note that as $\Delta h<\Delta H$, several attitude steps are required to cover one single orbital step. Therefore, the number of evaluations of the orbital (or slow) dynamics is smaller than that of the attitude (or fast) dynamics. Finally, note that, because the method is adaptive, it is possible that in order to not surpass the allowed truncation error, $\Delta H$ shall be reduced. 


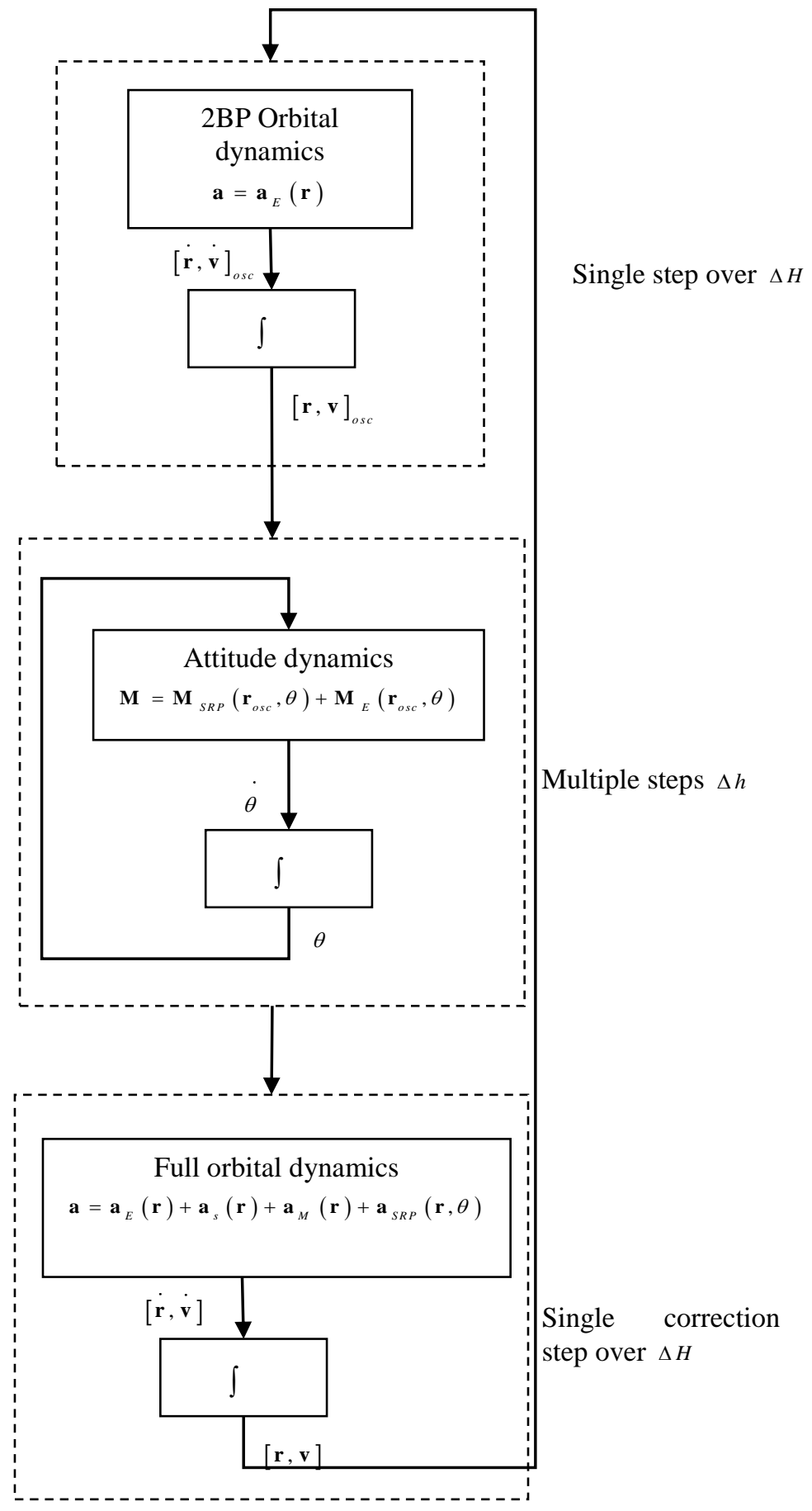

Fig. 68. Flow diagram of decoupled approach. 


\subsubsection{Averaged approach}

In both previous approaches (coupled and decoupled propagation) the attitude has to be propagated along time with the high frequency. This might lead to a fast degradation of the accuracy due to the amount of steps. Averaging the fast dynamics, instead, allows eliminating the fast frequencies completely. While the simulation time decreases, errors are introduced due to the averaging process, and depending on the selected averaging method itself.

Once the averaging law is used, the attitude motion is essentially not integrated, and only its net effect on the orbit is included in the orbital dynamics, in terms of accelerations:

$$
\mathbf{a}=\mathbf{a}_{E}(\mathbf{r})+\mathbf{a}_{s}(\mathbf{r})+\mathbf{a}_{M}(\mathbf{r})+\overline{\mathbf{a}}_{S R P}(\mathbf{r}, \bar{\theta})
$$

Where it was made explicit that the SRP term $\overline{\mathbf{a}}_{S R P}(\mathbf{r}, \bar{\theta})$, responsible for the attitude-orbit coupling, is averaged over the attitude. Note that, in this particular case of the SRP, not only the area-to-mass ratio has to be averaged, but also the direction of the solar radiation pressure force.

Averaging can be obtained in different ways. In a three-dimensional case of randomly tumbling object, and in absence of further data, it is reasonable to assume that the object will spend the same time in each one of all possible attitude states, and therefore the resulting average exposed area-to-mass ratio is simply the average of the area-tomass ratio of each possible attitude over time, and the direction of the SRP force is in average pointing in the direction opposite to the sun. However, on another assumption, the debris might be spinning around one axis, with no or small precession and nutation. In this case, the direction of the average SRP force might not point away from the sun, but instead it has to be evaluated through averaging process.

For this $2 \mathrm{D}$ test case, the value of the AMR will be estimated as the average between the maximum AMR (flat plate perpendicular to sun line), and the minimum AMR (i.e. zero when the flat plate is aligned with the sun direction) that the debris can adopt in any angular orientation. Therefore, the average SRP force is in the sun direction only.

\subsection{Results}

Three defferent test cases are performed, in which the integration was run using a different value of $d: 0.01$ (1\% of object surface is non-reflective), $0.001(0.1 \%)$ and $0.0005(0.05 \%)$. Table 12 , Table 13 and Table 14 show the results for each one of these cases. Essentially different values of $d$ change the amount of torque forcing the attitude motion, and therefore the typical frequency of the oscillation itself.

Each of the test case was perfomed in the following way: first, the reference orbit and attitude time histories were computed. This was done by integrating the fully coupled equations of orbit and attitude with a relative tolerance of 10E-16. The tables show the number of function evaluations necessary to complete the integration, as a reference (Full problem NFEVAL). Then, the three different approaches were run on the problem: fully coupled, decoupled and averaged. For the three approaches, the relative tolerance RelTol was tuned such to reach a similar value of the final position error, defined as: 


$$
E R R O R=\frac{\left|\mathbf{r}_{\text {true }}\left(t_{f}\right)-\mathbf{r}\left(t_{f}\right)\right|}{r_{0}}
$$

that is, the difference between the position at the final time resulting from the reference orbit calculation and the same resulting from using each one of the three approaches. The value of ERROR is shown in the tables. In this way, all three approaches find the orbit with similar accuracy, and hence the number of function evaluations that were used can be compared. The fully coupled approach evaluates the orbit and attitude dynamics simultaneously. Instead, the decoupled approach evaluates the orbit and the attitude dynamics separately, and therefore the number of function evaluations for each one of these (NFEVAL_O and NFEVAL_A respectively). The averaged approach does not solve the attitude motion, and therefore only the orbital dynamics is evaluated.

Table 12. $d=0.01(1 \%)$

\begin{tabular}{lccc}
\hline \multicolumn{2}{l}{ Full problem NFEVAL } & & $1,192,672$ \\
\hline & Coupled & Decoupled & Averaged \\
\hline RelTol & $5 \mathrm{e}-14$ & $1.0 \mathrm{E}-14$ & $1 \mathrm{E}-18$ \\
NFEVAL_A (c2) & 639,834 & 761,605 & $/$ \\
NFEVAL_O (c1) & 278,837 & 92,989 \\
ERROR & $1.139 \mathrm{E}-07$ & $1.250 \mathrm{E}-07$ & $1.56 \mathrm{E}-2$ \\
\hline
\end{tabular}

Table 13. $d=0.001(0.1 \%)$

\begin{tabular}{lccc}
\hline \multicolumn{2}{l}{ Full problem NFEVAL } & & 367,835 \\
\hline & Coupled & Decoupled & Averaged \\
\hline RelTol & $1 \mathrm{E}-13$ & $1.1 \mathrm{e}-14$ & $1 \mathrm{E}-18$ \\
NFEVAL_A (c2) & 183,703 & 230,464 & $/$ \\
NFEVAL_O (c1) & 110,838 & 94,315 \\
ERROR & $1.34 \mathrm{e}-08$ & $2.378 \mathrm{E}-08$ & $1.56 \mathrm{E}-2$ \\
\hline
\end{tabular}

Table 14. $d=0.0005(0.05 \%)$

\begin{tabular}{lccc}
\hline \multicolumn{2}{l}{ Full problem NFEVAL } & & 256,412 \\
\hline RelTol & Coupled & Decoupled & Averaged \\
NFEVAL_A (c2) & $2 \mathrm{E}-14$ & $1 \mathrm{E}-14$ & $1 \mathrm{E}-18$ \\
NFEVAL_O (c1) & 151,450 & 160,095 & $/$ \\
ERROR & $2.020 \mathrm{E}-08$ & $2.371 \mathrm{E}-08$ & $1.56 \mathrm{E}-2$ \\
\hline
\end{tabular}

Focusing on the case $d=0.01$ first (Table 12), the fully-coupled approach requires 639,834 function evaluations to achieve a relative error of 1E-7. The decoupled approach requires 761,605 attitude equation evaluations and 278,837 orbit equation evaluations. The fact that the attitude requires a higher number of function evaluations is to be expected, its dynamics is faster, and treated as such by the algorithm. The decoupled approach requires, overall, more function evaluations than the fully-coupled approach, however it is expected that the fully-coupled dynamics are more complex (and therefore more computationally demanding) than the attitude or orbit alone. If, for example, we assume that the computational time of a single evaluation of the fully coupled dynamics is unitary, and equally split between calculation of the orbit and the attitude, then in a first approximation we can say that the computational time for each evaluation of each decouples dynamics is 0.5 . In this case, the computational effort of the decoupled 
approach would be 520,221 (compared to 639,834 of the fully coupled approach), which corresponds to a saving of about $18 \%$ of the computational time. These figures are highly dependent on the complexity of evaluating the attitude dynamics with respect to the orbital dynamics. If evaluating the attitude differential equations results to be much more expensive than evaluating the orbit equations, then the decoupled approach becomes less convenient, or even inconvenient, with respect to the fully-coupled approach.

Finally, the averaged approach cannot reach the required position error, stopping at $1.56 \mathrm{E}-2$ even if the relative tolerance is set to $1 \mathrm{E}-18$. This is certainly due to the error introduced by not modelling the attitude dynamics at all. However, this approach requires 92,989 orbit function evaluations, which is only a small fraction of what needed by the other two approaches: if high accuracy is not the main requirement, then the averaged approach is without doubt the most efficient. In addition, a different averaging could lead to a smaller error, at no or small additional computational cost.

The other test cases, with different values of $d$, show a similar trend, however as the typical frequency of the attitude dynamics lowers, the advantage of the decoupled approach over the fully coupled approach reduces. 


\section{Conclusions}

This report has presented two approaches to model and integrate deformable, highly reflective membranes in Earth orbits. One is based on modelling the flexibility of the membrane as an Euler-Bernoulli truss, the second discretizes the membrane with lump masses connected by rods. The former assumes implicitly small displacements, which are unrealistic for large, thin membranes like MLI sheets, while the latter models arbitrarily large deformations.

Both approaches assume that the membrane can fold along one folding line, which remains fixed on the membrane. However, both approaches can be extended by considering more folding lines, at additional computational cost.

Both approaches were used to predict the orbit of a typical GEO debris, and compared with other propagation methods using rigid-body approximation, and showed sensible differences in the evolution of the orbital parameters, particularly inclination and eccentricity.

The linear approach is somewhat intrinsically limited due the fact that large displacements cannot be correctly model large membranes, whose deformations are clearly not limited in principle. The multi-body approach overcomes this limitation, at the cos of additional computational effort.

Earth gravity (up to J2), SRP, eclipses, gravitational attraction of sun and Moon, self-shadowing effects were all considered in the propagation. However, as it was mentioned in the introduction, because MLI membranes are aluminized and hence conductive, self-induced currents are also an important source of internal forces that contribute to the attitude dynamics and the deformation of the sheet.

Despite all flexible-body methods were matched to equivalent rigid-body approximations, and the results of the orbital integration were compared, no validation of either approach was done using real space debris data from empirical observations. Potentially, light curves of flexible debris could be estimated by considering the relative position and attitude with respect to the sun and an observer on the Earth. Simulations could be carried out assuming that a particular debris object is a deformable MLI sheet, and then matching the simulated attitude/deformation motion with the observed light curve. However, these would depend on extremely precise initial conditions, as well as characteristics (size, thickness, material) of the debris sheet which are not available, making this type of validation extremely difficult.

In addition, the multi-body approach makes use of discrete rotational springs and dampers to account for the bending stiffness of the membrane. The spring and damper constants were estimated through semi-empirical formulas, based on the data of the materials of the typical MLI membranes. These estimations, however, can be wrong, because of the extremely thin nature of the membrane itself. An experiment could be set up, in order to have a better estimation of these parameters. This is sketched in Fig. 69: an MLI sheet is held hanging on the top wall of a vacuum chamber. The sheet is then displaced from its equilibrium position along the vertical, and left swinging due to gravity. The amplitude and frequency of oscillation over time (monitored through a glass window) are related to 
the elastic properties of the membrane. The vacuum chamber ensures that the effect of the air friction is negligible. The spring and damper in the multi-body model can then tuned such that the response of the model matches that of the real membrane.

In a second set up, a beam of light can light the membrane through the glass window, and displacement of the MLI membrane due to SRP can be registered, with respect to the local vertical. According to preliminary calculations, this could be in the order of microns or fractions of a micron, hence highly precise and sensitive sensors have to be employed, to measure the displacement without contact. This variant of the experiment might allow to compute the effective SRP force on the membrane, once the light flux through the window is known.

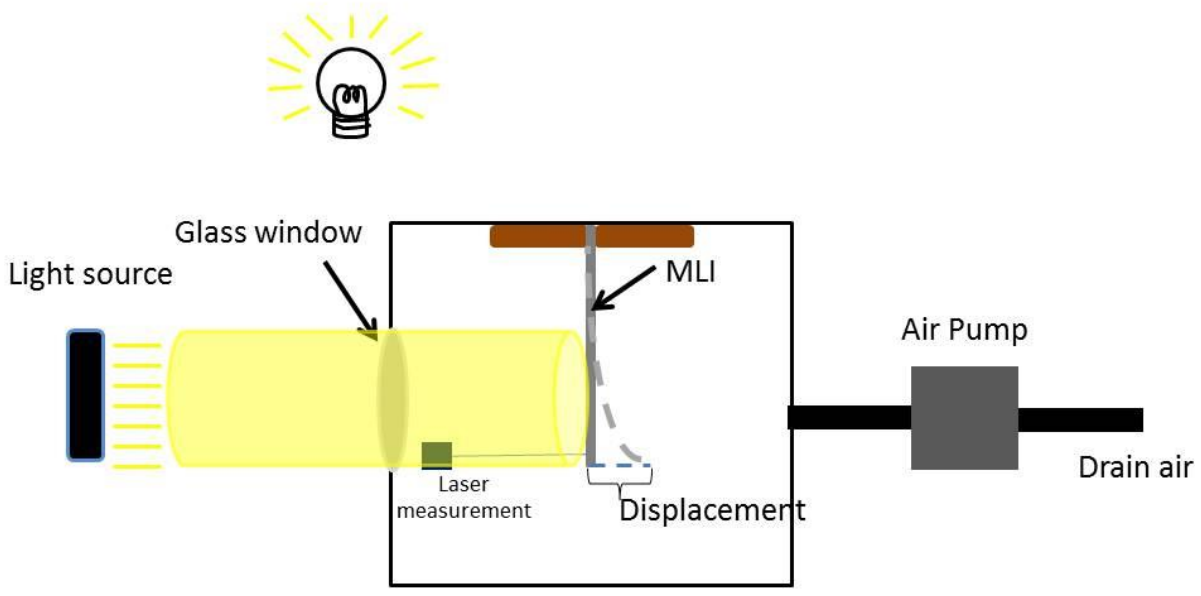

Fig. 69 Experimental set up for determination of MLI properties in a vacuum chamber.

Finally, a numerical integrator was developed to integrate two coupled dynamics with different time-scales. The principle is that the slow dynamics can be integrated with a longer time-step with respect to the fast one, and its states are estimated in order to resolve the fast dynamics at each evaluation. The integrator is of general purpose, but it was applied to a flat plate with fast spinning dynamics and slow orbital dynamics.

It was shown that a decoupled approach can have some advantages in computational time, when integrating coupled attitude and orbital dynamics, but with the present algorithm, the advantage is dependent on the relative time spent on the two dynamics. Further study is necessary to:

- Optimize the decoupled approach, in particular with respect to the selection of the tolerances and the step sizes internally.

- Test the decoupled approach using more complete models for the orbital and attitude dynamics. This will also allow having a realistic estimation of the time spent on the evaluation of each one of the dynamics.

- Include three dynamics in the decoupled approach (orbit, attitude and deformation), or alternatively embed the deformation dynamics in the attitude dynamics. 


\section{Appendix A - ABAQUS modelling for validation}

In this section, we use finite element method (FEM) to build a model simulating the membrane structure at GEO and analysis its dynamic behaviors under the load of SRP. As for material properties, we used the most common membrane-type space debris the separated multi-layer insulation (MLI). We used a commercial FEM software ABAQUS to do most of the analysis job and get the result, some of which were used as a validation of the MATLAB of the deformable debris object.

\subsection{Basic ideas of FEM}

\subsubsection{Classification and application}

The FEM is a very powerful tool for solving numerous engineering problems and widely used in mechanics analysis, thermal analysis and many other branches of industry. In this report we focus on mechanics analysis.

Considering the fact that load applied on the object of study vary, people sort the problem into static problem and dynamic problem, then use different governing equations to describe the problem. In static problem, a structure is under static load and usually reaches equilibrium condition. Typical static problem includes structure distortion analysis, certain structure component stress and strain condition analysis, etc. [51]. In dynamic problem, however, the direction, value and application point of the load can all be functions of time, and the FEM is used to calculate the forced response of the body. Typical dynamic problem includes forced frequency analysis of aircraft and spacecraft components, analysis of impact problem and time dependent analysis of structure dynamic, etc. [51].

\subsubsection{General theory}

The basic principles underlying the finite element method are not complex. When analyze the distribution of an unknown variable on an object, taking displacement or stress in elasticity problem as an example, we follow the steps below.

The first step is discretization of the problem. The real object with infinite degree of freedom is divided into a series of regular shaped and manageable pieces called "elements", and they are interconnected to each other at joints called "nodes". In FEM every element represents a discrete portion of the real object, and our interested variable, such as displacement, is calculated only at the nodes of the element. Then, at any other point in the element, the displacements are obtained by interpolating from the nodal displacements in a certain manner, such as linear or quadratic polynomial and trigonometric function, which is usually determined by the element type we choose and the number of nodes used in the element. Therefore, once we get the value of the variable at every node of every element, the variable distribution through every element, and further the whole body, is adequately approximated.

All structures in real world are three-dimensional, however, considering memory usage, computing time and the property of the problem itself, proper simplification is essential and reasonable. For example, it is economical to develop a kind of element that can carry only tensile or compressive loads without resistance to bending and have 
only translational degrees of freedom at each node, to discretize a long, axisymmetric part under only axial load, like a part of the scaffold. Hence, different types or families of element were developed for different simulation and result demand, and the truss element is exactly the element type stated in the example above. Fig. 70, from [52].

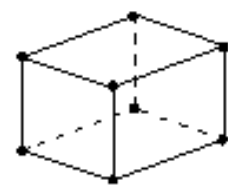

Continuum (solid) elements

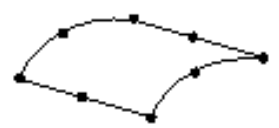

Membrane elements

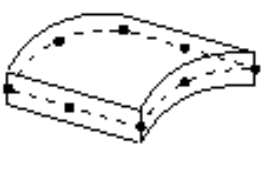

Shell elements

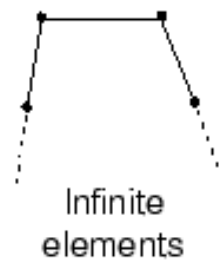

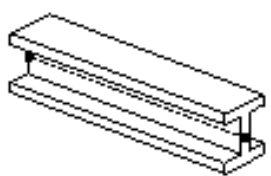

Beam elements
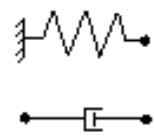

Springs and dashpots

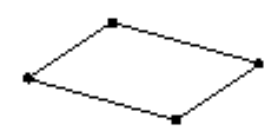

Rigid elements

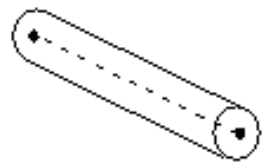

Truss elements

Fig. 70. Most element families currently available in commercial FEM software for mechanic analysis (from [52])

With different element families to choose and adjustable nodes to put on a certain element, the discretization job becomes more flexible. In mechanic simulation, the fundamental variables are the translational degrees of freedom (DOF) in three directions and rotational DOF in three directions. In order to get the required variables, such as stress strain or deformation of the object, they should be calculated step by step, node by node during an analysis producer. Some element families developed for certain kinds of problems, however, eliminate some unnecessary DOF to simplify the model and accelerate calculation. What if the element family is suitable but a more accurate distribution of the variable is desired? A more-node-type element is developed to increase the interpolation order without changing other properties of the same element family, as illustrated in the examples in Fig. 71 (from [52])

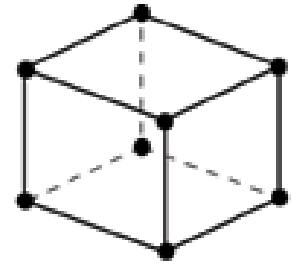

(a) Linear element (8-node brick, C3D8)

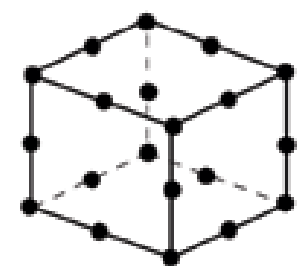

(b) Quadratic element (20-node brick, C3D20)

Fig. 71. Finite element types (from [52]).

- Elements that have nodes only at their corners, such as the 8-node brick shown in Figure 2(a), use linear interpolation in each direction and are often called linear elements or first-order elements.

- Elements with mid-side nodes, such as the 20-node brick shown in Fig. 71 (b), use quadratic interpolation and are often called quadratic elements or second-order elements. 
The next step after the discretization is to set up the basic governing equation, usually called finite element equation. Theoretically, the finite element equation should contains all the information of the system that we know and want to know, for instance applied load, boundary condition and displacements. Finite element equations differ in formulation for different problems, however, the principle to derive it is the same, to set up the equations on every single element, and then assemble them to a whole.

Our problem belongs to the elasticity problem. In elasticity analysis, with the principle of superposition and several basic type of load, namely concentrated force, distributed force or pressure, body force (inertia or gravitational) and initial strain $\sigma 0$, any complex force condition can be imitated and then substituted into the finite element equations to yield the nodal displacements. Then the strain, stress and other variables in elasticity analysis is easily to get from the nodal displacements.

The derivation of the general finite element equation for elasticity problem is given by the method of minimization of the potential energy.

The potential energy $\Pi$ equals the strain energy $\Lambda$ in the body less the work done by the external loads acting on the body:

$$
\Pi=\Lambda-W
$$

In a differential element of volume $d V$, the strain energy $d \Lambda$ is found from

$$
d \Lambda=\frac{1}{2}\{\varepsilon\}^{T}\{\sigma\}-\frac{1}{2}\left\{\varepsilon_{0}\right\}^{T}\{\sigma\}
$$

Where, $\{\varepsilon\}$ is a vector of the total strain; $\left\{\varepsilon_{0}\right\}$ is a vector of initial strain; and $\{\sigma\}$ is a vector of the stress components.

The total strain energy for a finite volume is therefore calculated by integrating (6) though the volume. Hence

$$
\Lambda=\int_{V} \frac{1}{2}\left(\{\varepsilon\}^{T}\{\sigma\}-\left\{\varepsilon_{0}\right\}^{T}\{\sigma\}\right) d V
$$

According to basic elastic theory, the $\operatorname{strain}\{\varepsilon\}$ and stress $\{\sigma\}$ have relation as below

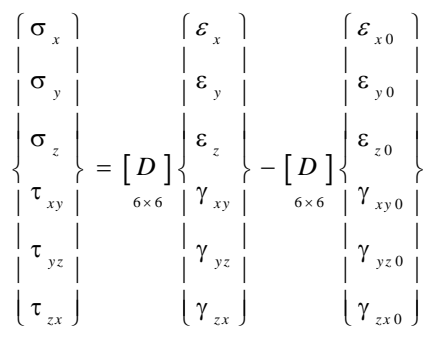

Where [D] is elastic coefficient matrix, determined by material properties, like Young's modulus E and Poisson Ratio $v$.

Therefore, if we substitute (8) into (7) we can get

$$
\Lambda=\frac{1}{2} \int_{V}\left(\{\varepsilon\}^{T}[D]\{\varepsilon\}-2\{\varepsilon\}^{T}[D]\left\{\varepsilon_{0}\right\}+\left\{\varepsilon_{0}\right\}^{T}[D]\left\{\varepsilon_{0}\right\}\right) d V
$$


Another basic principle from elastic theory is that the strains are partial differentiations of displacements $\{u\}=\left\{\begin{array}{lll}u_{1} & u_{2} & u_{3}\end{array}\right\}^{T}$ in three directions.

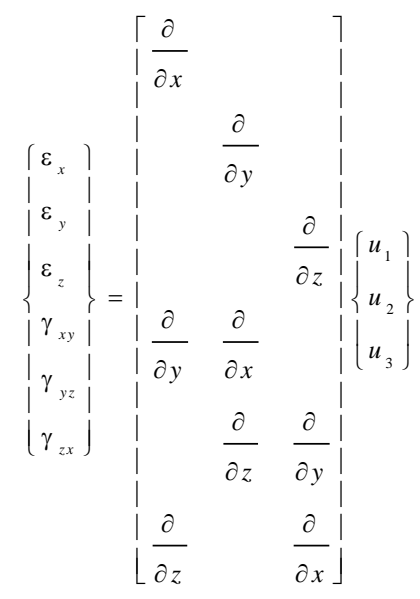

As mentioned before, in FEM the displacements throughout each element are interpolated from the nodal displacements in certain manners.

$$
\{u\}=[N]\{U\}
$$

Where $\{u\}$ will represent the displacements of any point on an element, $[N]$ is the shape function matrix determined by the interpolation function and $\{U\}$ is a row vector of nodal displacements.

Then the strains can also equated to the nodal displacement by

$$
\{\varepsilon\}=[B]\{U\}
$$

Eq 2

Compare $\mathrm{Eq} 1$ and $\mathrm{Eq} 2$, we can find that $[\mathrm{B}]$ is a matrix derived by the proper differentiation of the shape function matrix $[N]$.

Substitution of Eq 2 into Eq 1 gets the strain energy for a single element $\Lambda^{(e)}$, namely

$$
\Lambda^{(e)}=\frac{1}{2} \int_{V^{(e)}} \begin{aligned}
& \left(\left\{U^{(e)}\right\}^{T}\left[B^{(e)}\right]^{T}\left[D^{(e)}\right]\left[B^{(e)}\right]\left\{U^{(e)}\right\}-\right. \\
& \left.2\left\{U^{(e)}\right\}^{T}\left[B^{(e)}\right]^{T}\left[D^{(e)}\right]\left\{\varepsilon_{0}^{(e)}\right\}+\left\{\varepsilon_{0}^{(e)}\right\}^{T}\left[D^{(e)}\right]\left\{\varepsilon_{0}^{(e)}\right\}\right) d V
\end{aligned}
$$

In order to get the potential energy of a single element, the work done on the single element by external load is then needed. As mentioned before, no matter how complex a real load may be, its work can be simplified into three basic parts that are the work done by concentrated nodal force, by pressure and by body force. All those work is simply the magnitude of the forces multiplying the distance moved on respective direction.

Therefore, if the vector of the concentrated nodal loads is $\left\{F^{(e)}\right\}$, then the work done should be

$$
W_{C}^{(e)}=\left\{U^{(e)}\right\}^{T}\left\{F^{(e)}\right\}
$$

Assume $\left\{p_{x}^{(e)} p_{y}^{(e)} p_{z}^{(e)}\right\}^{T}$ is the distributed pressure loads parallel to the coordinate axes and considered positive when acting in the positive coordinate direction, then the work done by pressure is

$$
W_{P}^{(e)}=\int_{S}\left\{U^{(e)}\right\}\left[N^{(e)}\right]{ }^{T}\left\{\begin{array}{l}
p_{x}^{(e)} \\
p_{y}^{(e)} \\
p_{z}^{(e)}
\end{array}\right\} d S
$$

Eq 5

Assume the body force is $\left\{X^{(e)} Y^{(e)} Z^{(e)}\right\}^{T}$ per unit volume, then the work done by body force is 


$$
W_{B}^{(e)}=\int_{V^{(e)}}\left\{U^{(e)}\right\}^{T}\left[N^{(e)}\right]^{T}\left\{\begin{array}{l}
\left.X^{(e)}\right\} \\
Y^{(e)} \\
Z^{(e)}
\end{array}\right\} d V
$$

Eq 6

Therefor the potential energy of a single element is given by Error! Reference source not found. and Eq 3 to Eq 6, namely

$$
\Pi^{(e)}=\Lambda^{(e)}-W_{C}^{(e)}-W_{P}^{(e)}-W_{B}^{(e)}
$$

If the real object is discretized into $N$ elements, then the potential energy of the whole system is simply the sum of every single element

$$
\Pi=\sum_{e=1}^{N} \Pi^{(e)}
$$

For the object under load to be in equilibrium, its potential energy must reach a minimum. Hence for all degrees of freedom, namely displacements for every node on all three possible directions, must obey

$$
\begin{aligned}
& \frac{\partial \Pi}{\partial u_{1}}=\frac{\partial \Pi}{\partial u_{2}}=\cdots \frac{\partial \Pi}{\partial u_{n}}=0 \\
& \text { or } \frac{\partial \Pi}{\partial\left\{U_{S}\right\}}=0
\end{aligned}
$$

Where we assume $n$ is the total degrees of freedom, $\left\{U_{s}\right\}$ is the vector consists of all elemental displacement vectors. Calculate out Eq 9 we can actually get $n$ equations with $n$ unknowns

$$
\begin{aligned}
\frac{\partial \Pi}{\partial\left\{U_{S}\right\}}= & \sum_{e=1}^{N}\left[\int_{V^{(e)}}\left(\left[B^{(e)}\right]^{T}\left[D^{(e)}\right]\left[B^{(e)}\right]\right) d V\right]\left\{U_{S}\right\} \\
& -\sum_{e=1}^{N}\left[\int_{V^{(e)}}\left(\left[B^{(e)}\right]^{T}\left[D^{(e)}\right]\left\{\varepsilon_{0}^{(e)}\right\}\right) d V+\int_{V^{(e)}}\left[N^{(e)}\right]^{T}\left\{\begin{array}{l}
\left.X^{(e)}\right\} \\
\left.Y^{(e)}\right\} d V \\
\left.Z^{(e)}\right\}
\end{array}\right.\right. \\
& \left.+\int_{S}\left\{U^{(e)}\right\}\left[N^{(e)}\right]^{T}\left\{\begin{array}{c}
p_{x}^{(e)}\{ \\
p_{y}^{(e)} \\
\vdots \\
p_{z}^{(e)}
\end{array}\right\} d S\right]-\left\{P_{S}\right\}=0
\end{aligned}
$$

To be simplify Eq 10 may be written as

$$
[K]\left\{U_{S}\right\}=\left\{F_{S}\right\}
$$

Eq 11

Where

$$
[K]=\sum_{e=1}^{N}\left[\int_{V^{(e)}}\left(\left[B^{(e)}\right]^{T}\left[D^{(e)}\right]\left[B^{(e)}\right]\right) d V\right]=\sum_{e=1}^{N}\left[K^{(e)}\right]
$$

Eq 12

$[K]$ is global stiffness matrix, $\left[K^{(e)}\right]$ is the individual stiffness matrix and the global force vector is 


$$
\begin{aligned}
&\left\{F_{S}\right\}=\sum_{e=1}^{N}\left[\int_{V^{(e)}}\left(\left[B^{(e)}\right]^{T}\left[D^{(e)}\right]\left\{\varepsilon_{0}^{(e)}\right\}\right) d V+\int_{V^{(e)}}\left[N^{(e)}\right]^{T}\left\{Y^{(e)}\right\} d V\right. \\
&\left.+\int_{S}\left\{U^{(e)}\right\}\left[N^{(e)}\right]\right\} \\
&\left.\left\{\begin{array}{l}
\left.p_{x}^{(e)}\right\} \\
\left\{p_{y}^{(e)}\right\} \\
\left.p_{z}^{(e)}\right\}
\end{array}\right\} d S\right]+\left\{P_{S}\right\}=\sum_{e=1}^{N}\left[F^{(e)}\right]
\end{aligned}
$$

Eq 13

Usually the individual element stiffness matrix and force vector for each element is solved first, and then they are assembled into the global matrix and vector. Certainly the symbol " $\sum$ " does not mean what it usually means. The individual stiffness matrixes are obtained in the local coordinate of every element, to assemble into the global matrix they should first be converted into global coordinate and then added to the proper locations in the global matrix according to its nodal association.

Now we have derived Eq 11 as the basic equation for finite element problem. It is the governing equation for elastic static analysis, however for dynamic analysis, which is exactly our situation, it is not comprehensive enough. There should be some other terms in the equation that can represent the features of dynamic problem, namely inertia and damping. So the basic equation of motion for the object becomes

$$
[M]\left\{U_{s}\right\}+[C]\left\{U_{s}\right\}+[K]\left\{U_{s}\right\}=\left\{F_{s}(t)\right\}
$$

Eq 14

In which, $[M]$ is mass matrix and $[C]$ is damping matrix, which are determined by the material properties, and $\left\{F_{S}(t)\right\}$ means the load may vary as time goes by.

After we have get the finite element equation for the problem the final step is to solve the equation, there are mainly two classes the direct methods and the iterative methods, both of which have been studied a lot in mathematics and numerical analysis, and most commercial FEM packages have integrated the latest achievements. We will not focus on the solution methods in this report.

\subsubsection{A brief introduction to ABAQUS}

ABAQUS, based on FEM, is a suite of engineering simulation programs, including: ABAQUS/Standard, a general-purpose finite element program; ABAQUS/Explicit, an explicit dynamics finite element program; ABAQUS/CAE, an interactive environment used to create finite element models, submit analyses, monitor and diagnose jobs, and evaluate results; and ABAQUS/Viewer, a subset of ABAQUS/CAE that contains only the postprocessing capabilities of the Visualization module. It is a general-purpose simulation tool that can be used in diverse areas, such as stress/strain analysis, heat transfer analysis, mass diffusion analysis, piezoelectric analysis, electromagnetic analysis, and fluid dynamics [6]. In ABAQUS/CAE there is an extensive elements library to model complex geometry and an extensive material library to simulate the behavior of most typical engineering materials.

Using ABAQUS to analyze a problem should follow three successive steps, preprocessing, simulation, and postprocessing, shown in Fig. 72 three steps to analyze problem by ABAQUS[6]: 


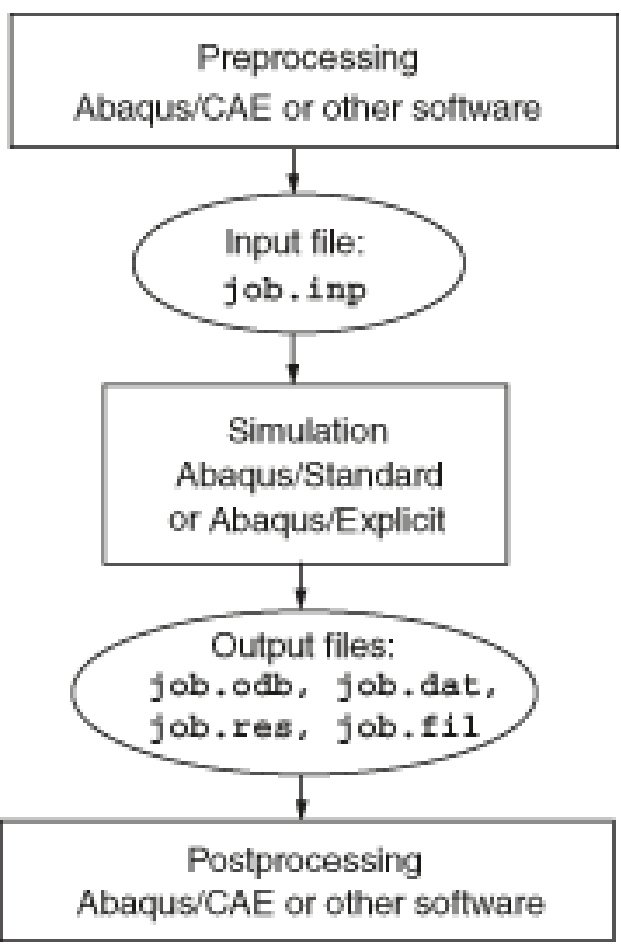

Fig. 72 three steps to analyze problem by ABAQUS

A basic concept in ABAQUS is the division of the problem history into "step" that is any phase of the history. Taking the simplest form in ABAQUS/Standard static analysis as an example, a "step" is a load change from one magnitude to another. In each "step" the user chooses a "procedure", defining the type of analysis to be performed during the step: static analysis, dynamic stress analysis, eigenvalue buckling, etc. The procedure choice can be changed from step to step in any meaningful way. Since the state of the model is updated throughout all analysis steps, the effects of previous steps are always included in the response in each new step.

ABAQUS/Standard provides both linear and nonlinear response options. Linear analysis is considered as linear perturbation analysis. This linear perturbation approach allows general application of linear analysis techniques in cases where the linear response depends on preloading or the nonlinear response history of the model [6].

In nonlinear analysis, which is our case, the goal is to obtain a convergent solution by a proper method at the minimum cost. So the solution will be developed by a series of "small" increments. For a successful FEM package, two aspects must be focused on: first, the method solving discrete equilibrium equation at each increment; second, the technique to setting the proper increment size. For solving the nonlinear equilibrium equations at each increment, many numerical methods, usually based on Newton method, are available for ABAQUS/Standard. Again taking our problem as an example, Modified Newton's method is just suitable. Other methods like Full Newton method, Approximate Newton method and Quasi-Newton method, etc. all have their suitable cases.

While setting the increment size, certainly ABAQUS will allow the direct user control of increment size where the user specifies the incrementation scheme. The automatic control of increment size, however, should manifest the value of ABAQUS more. This approach, in which user defines the step and specifies certain tolerances or error measures and then let ABAQUS automatically selects the increments as it develops the response in the step, is 


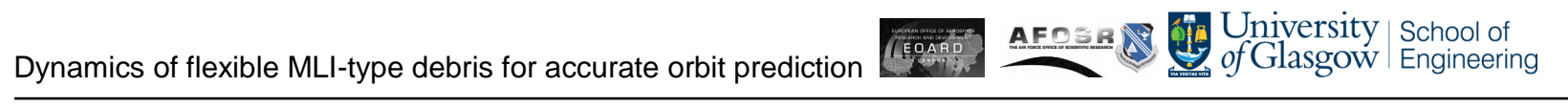

usually more efficient, since it is hard for user to predict the response ahead of time. Automatic control is particularly valuable in cases where the time or load increment varies widely through the step, such as creep, heat transfer, and consolidation [6].

Those are just brief introduction of ABAQUS, more detailed discussion on solving method and theory shall refer to ABAQUS user's Manual or other reference literature.

\subsection{Modeling and Result}

In this project, our work was built in ABAQUS/CAE and use ABAQUS/Standard to carry out the stress/displacement analysis. And the properties we used to model the space membrane structure, as we have mentioned in the first part of the report, are from a sheet of separated MLI and shown in the table below.

Table 15 properties and conditions for builing the model

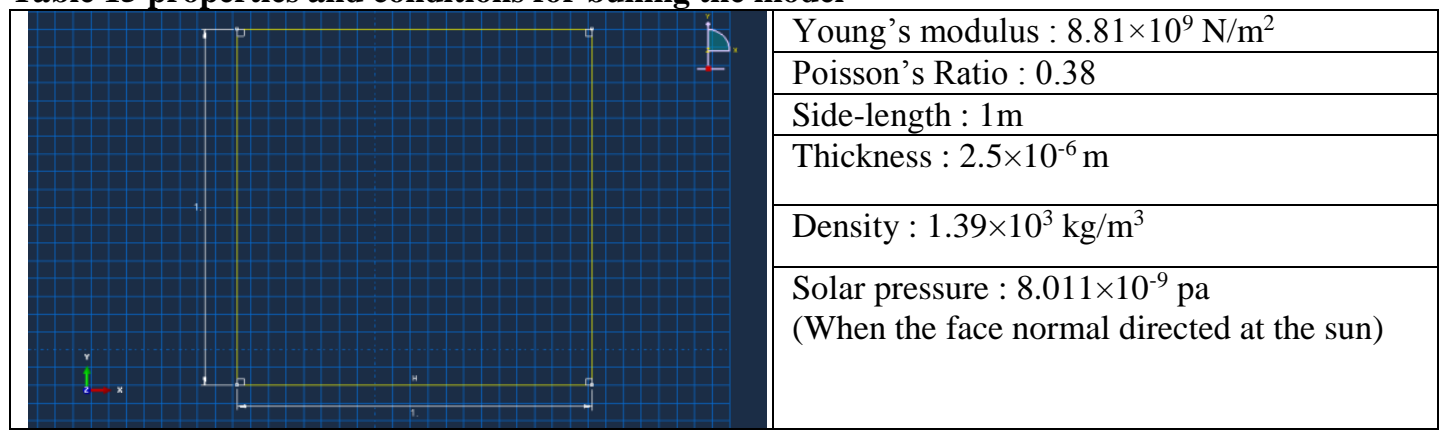

In our simulation there is a thin membrane under relatively small load changing its geometry slowly, and we are interested in the distribution of displacement, stress and strain. So based on these characteristics of our problem, the element type we chosen is linear (4-node) quadrilateral curved shell. It is a general-purpose element that can provide robust and accurate simulation for thin and or shell under various loads. When using this element, the membrane is assumed having no transverse shear locking, and unconstrained hourglass modes. This element considers finite membrane strains and the membrane kinematics are based on an assumed-strain formulation that provides accurate solutions for in-plane bending behavior.

There are three typical original membrane geometries designed to analyze its response under load in space (mainly solar pressure).

\subsubsection{Case 1, Flat plate under normal pressure}

Assume the face normal directed at the sun and the solar pressure applied for 1 second, shown in Fig. 73, the pink arrow represents the force vector. 


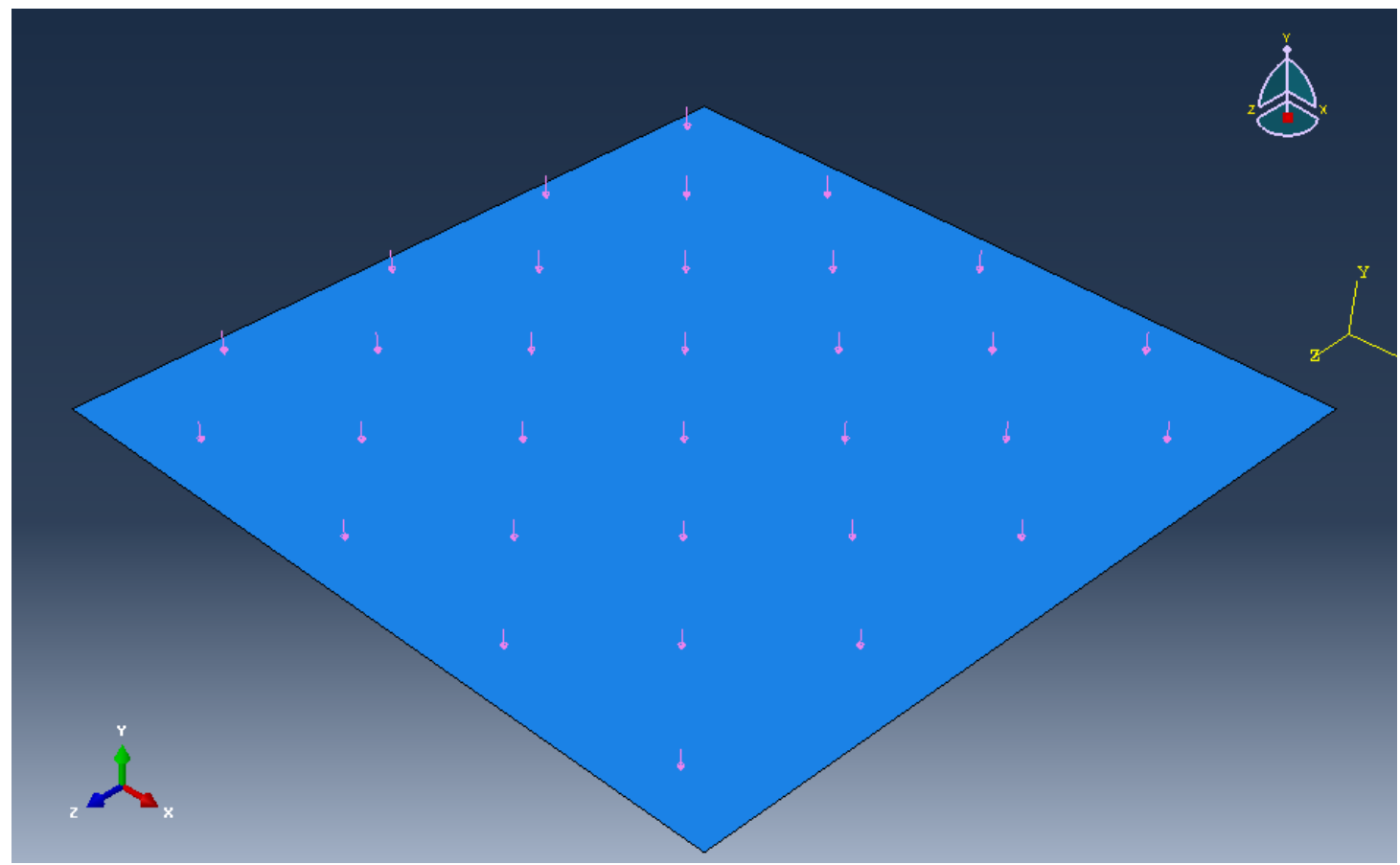

Fig. 73 force condition for case 1

And here is the simulation result of displacement from ABAQUS

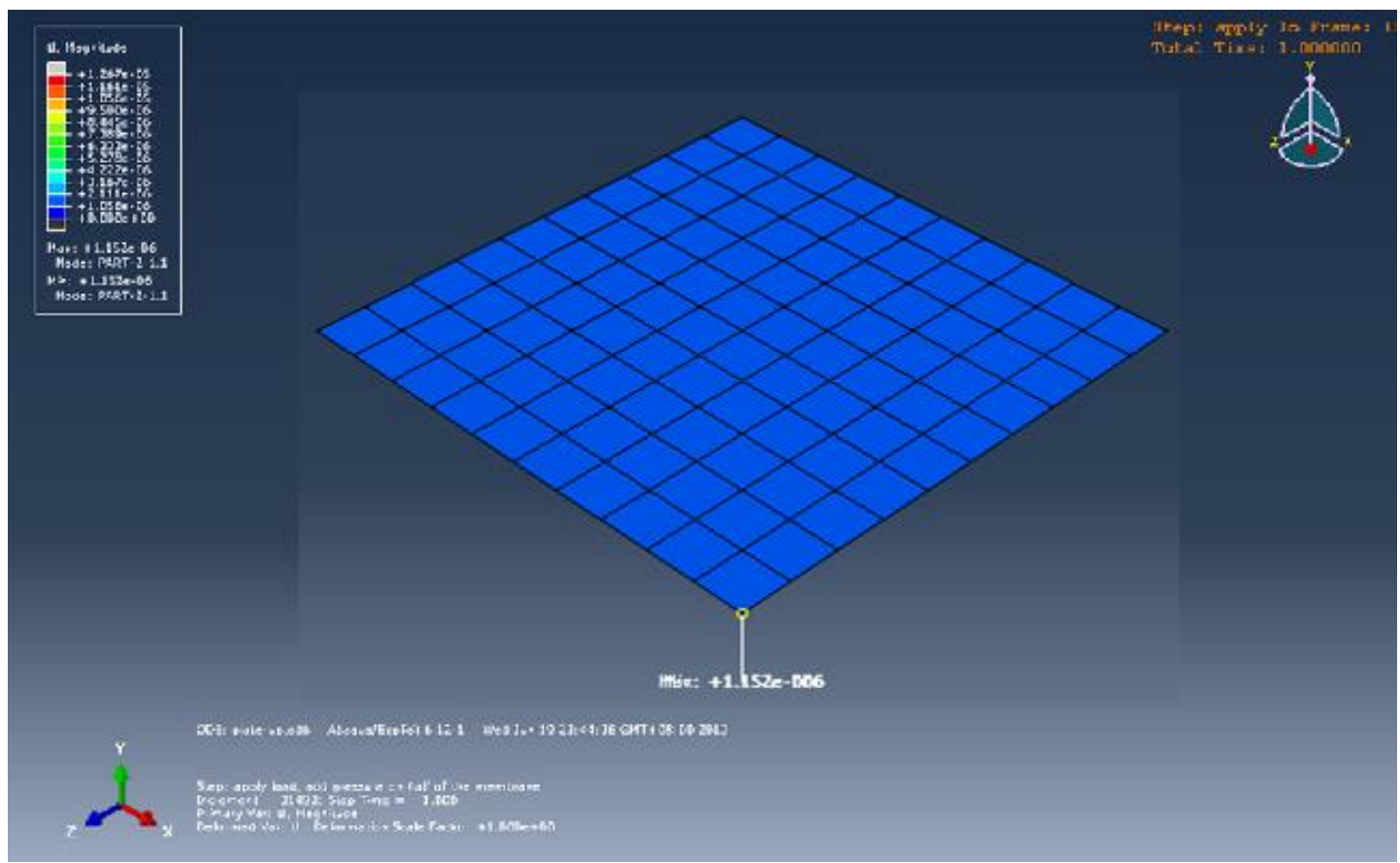

Fig. 74 displacement distribution for case 1

As we can see from Fig. 74 the whole body is in the same color, which means the displacement of every element is the same, as it marked out in white, $\max =\min =1.152 \mathrm{e}^{-6}(\mathrm{~m})$. As for the stress and strain, it is no surprise to find they are zero all around the plate. 
Actually, for this simplest situation, the solar pressure is added evenly to the surface, it is not hard to get the same conclusion based on Classical Kinematics Theory. For all elements, they have the same mechanics properties and the load situations, so after the period of time they should have the same movements. And the displacements of every element can be calculated out equally by Kinematics theory.

From Newton's laws of motion the acceleration equals:

$$
\mathrm{a}=\frac{F}{m}
$$

where: $\mathrm{F}=8.011 \times 10^{-9} \times 1 \times 1=8.011 \times 10^{-9} \mathrm{~N}$, is the total load applied on the body

$$
\mathrm{m}=2.5 \times 10^{-6} \times 1 \times 1 \times 1.39 \times 10^{3}=3.475 \times 10^{-3} \mathrm{~kg} \text {, is the total mass of the body. }
$$

So, $\quad \mathrm{a}=2.305 \times 10^{-6} \mathrm{~m} / \mathrm{s}^{2}$

Then, from the basic Kinematics equation:

$\mathrm{d}=\frac{1}{2} a t^{2}$

the displacement $\mathrm{d}=1.152 \times 10^{-6} \mathrm{~m}$.

The results of the same problem from two different methods are same, which to a certain extent showed the correctness of this method.

\subsubsection{Case 2, Right-angle membrane under constant solar pressure}

In this case we assume the original geometry for this membrane is not plate. As we can see in Fig. 75 it forms a right-angle at the middle point of edges. The solar pressure, added to the face-YOZ, will last for 60 seconds, and to make the simulation more factual its direction is defined with respect to the original body, which means the load always acts in a fixed global direction.

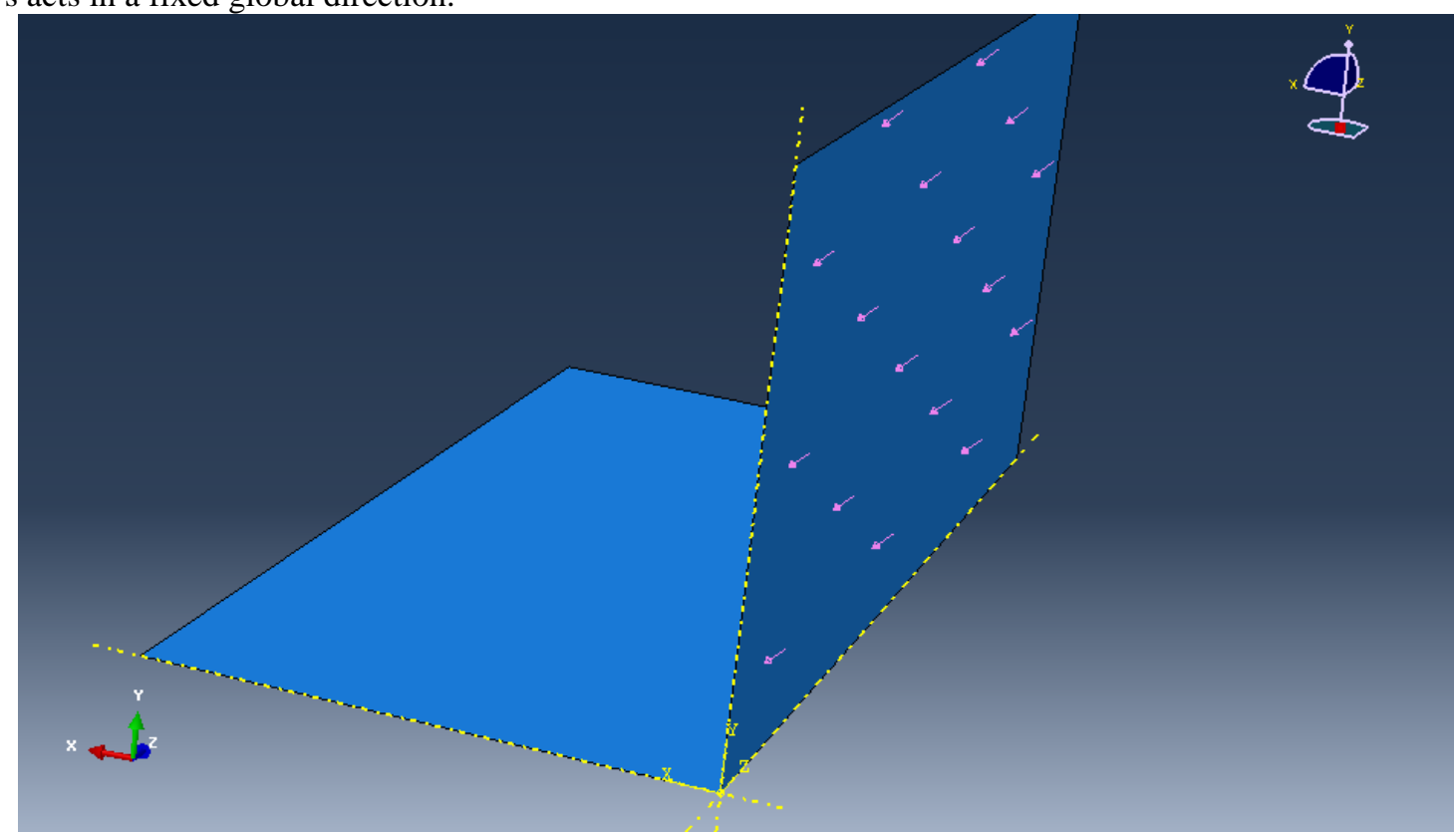

Fig. 75 force conditon for case 2

Here are the simulation results for this case. Fig. 76 shows displacement distribution of every node after 60 seconds. As it marked out, the maximum and minimum displacement located respectively at the upper edge and 
lower edge, $\max =3.822 \times 10^{-3}$, $\min =1.043 \times 10^{-3}$. Fig. 77 and Fig. 78 shows, respectively, the distribution of stress and logarithmic strain at 60 second, also marking out the max and min values, and still their contours pictures are same.

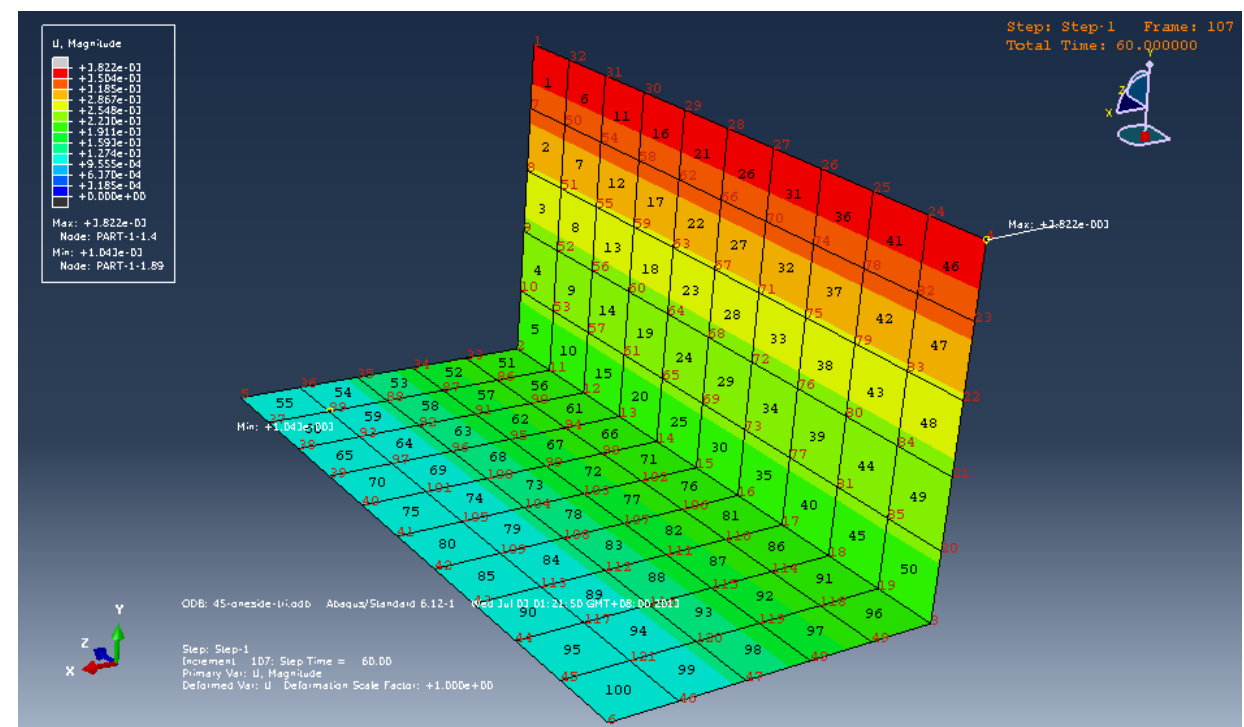

Fig. 76. displacement distribution for case 2

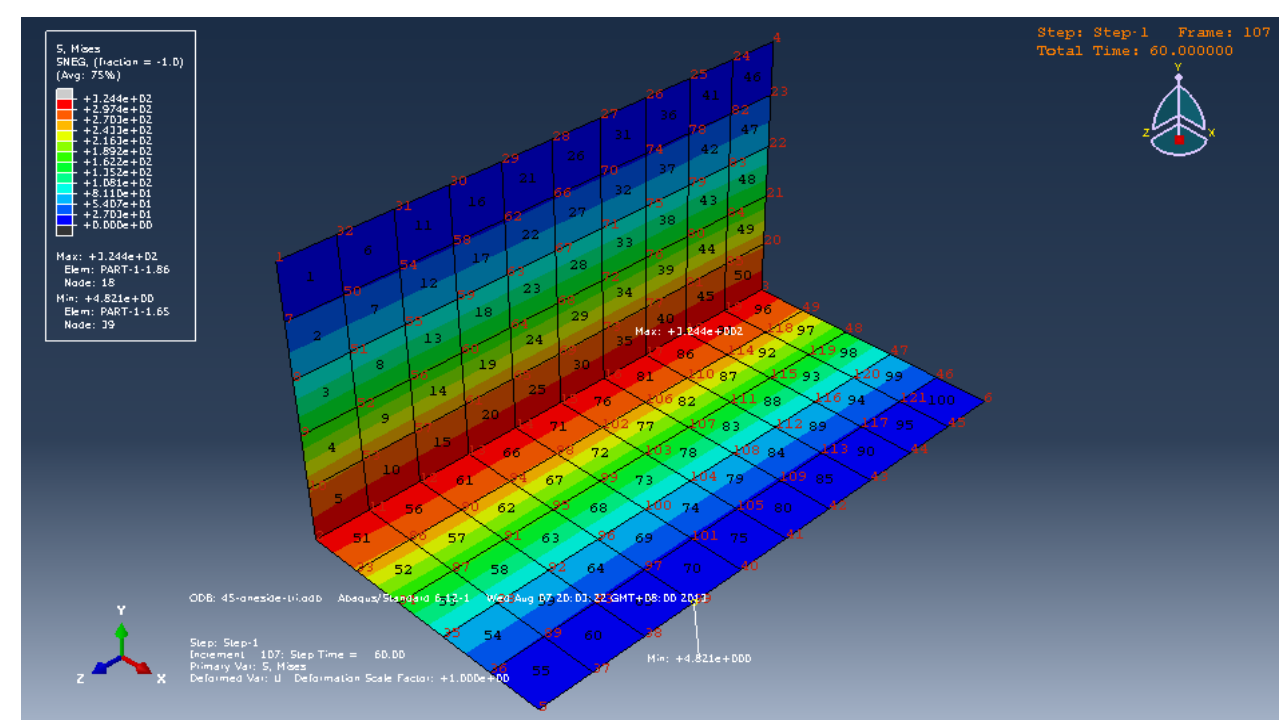

Fig. 77 stress distribution for case 2. 


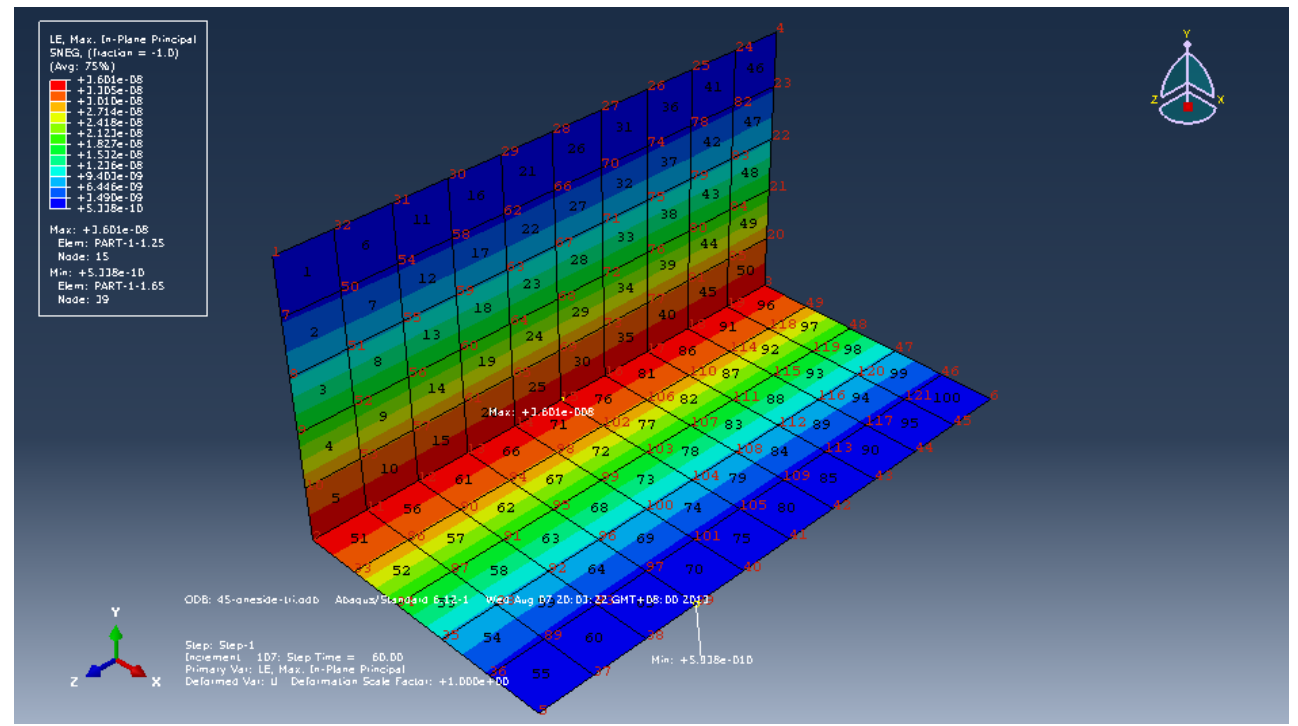

Fig. 78 strain distribution for case 2

In real situation the membrane is under a very complex force condition and the force applied on it may change as time goes by. So in the following case we simulate the situation that the membrane, in the same origin geometry of last one, was destabilized by a relatively large force in the first five seconds of the total duration of solar pressure.

\subsubsection{Case 3, Right-angle membrane under changeable load}

In this case, we assume in the first five seconds, beside the solar pressure, there is another pressure applied on the membrane. As shown in Fig. 79, the new arrows normal to the vertical half-face represent the new pressure and its magnitude is $10^{-5} \mathrm{pa}$.

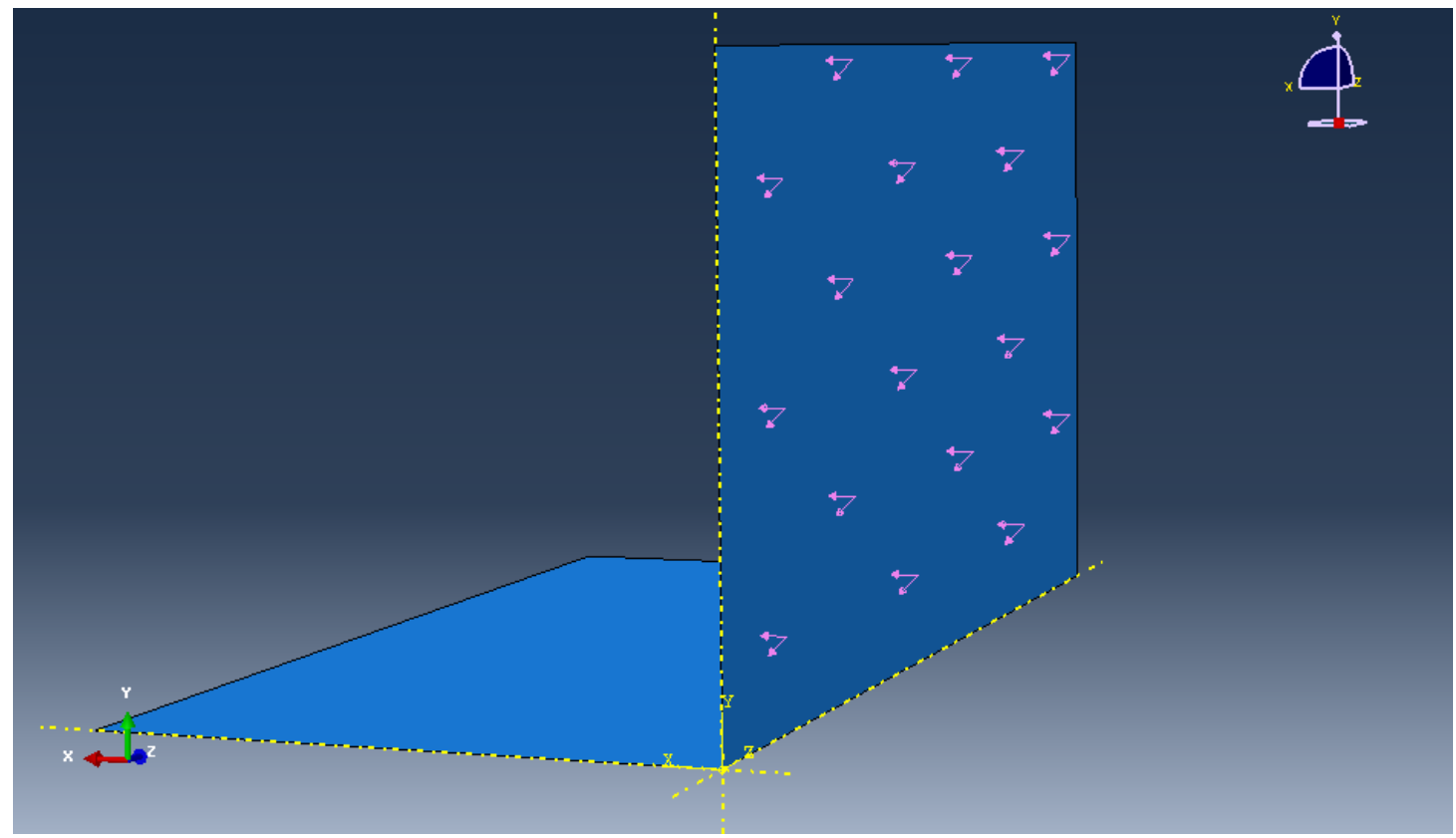

Fig. 79 force conditon for case 3 
Fig. 80 shows the displacement distribution under the new disturbing load, as we can see, the transparent body is the original body and the entity is how it deformed after 60 seconds. Obviously, this distinguishable deformation will have a complex influence on its motion characteristic. Fig. 81 is the stress contours picture for this case, and still the strain has the same contours picture as stress, which is Fig. 82.

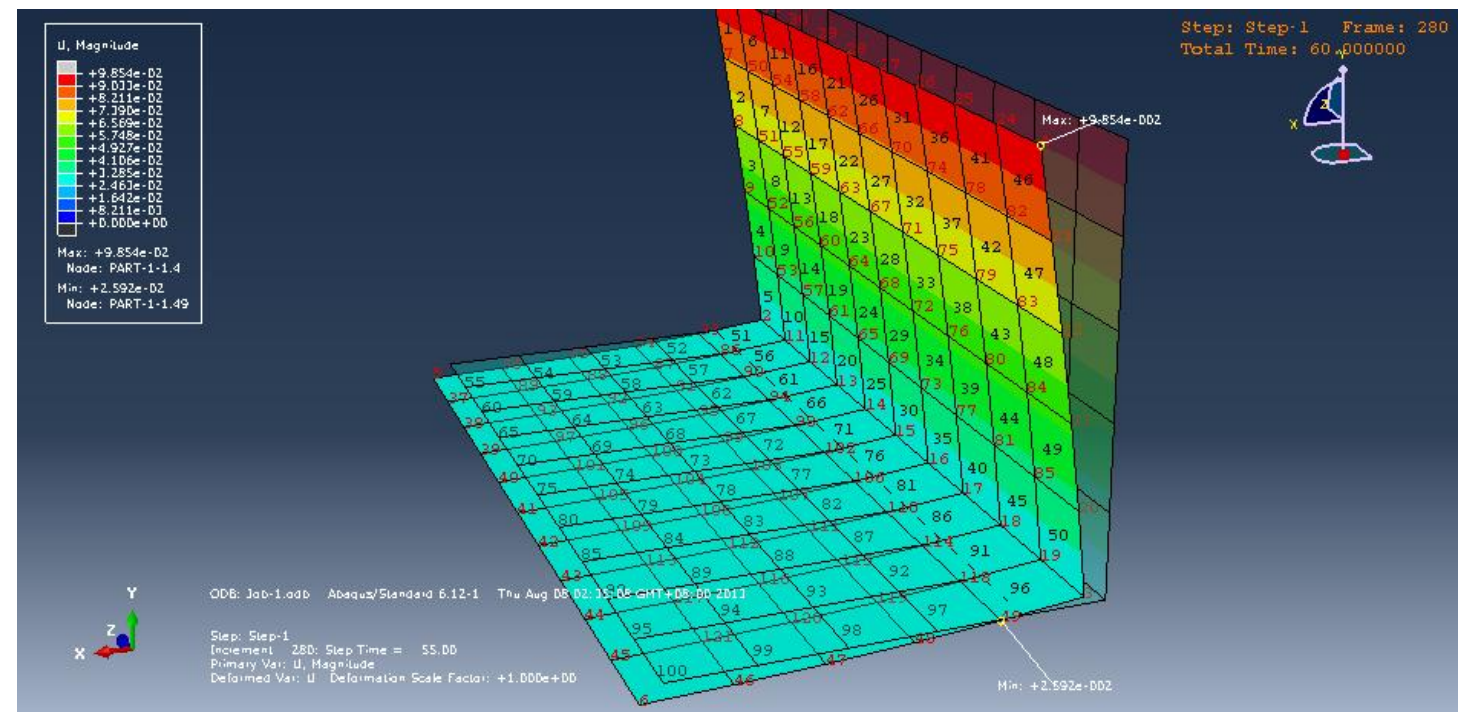

Fig. 80 displacement distribution for case 3

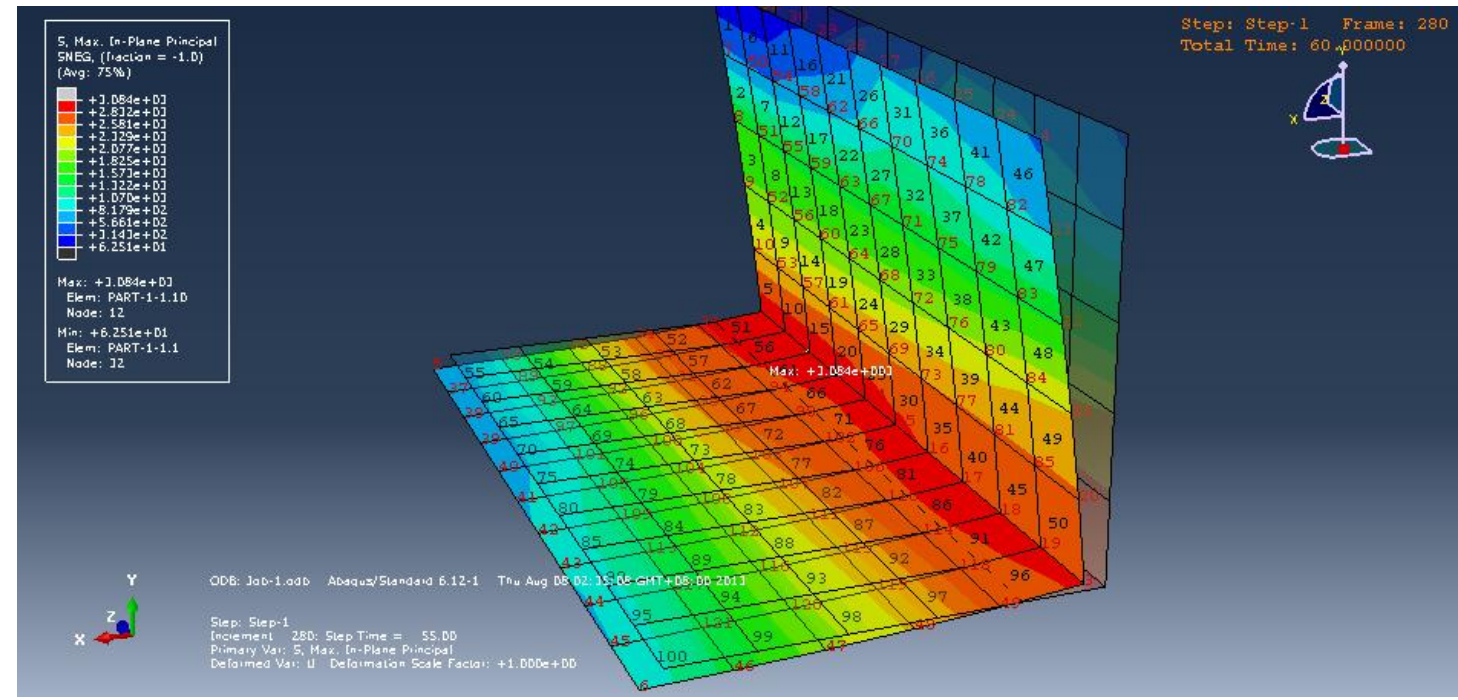

Fig. 81 stress distribution for case 3 


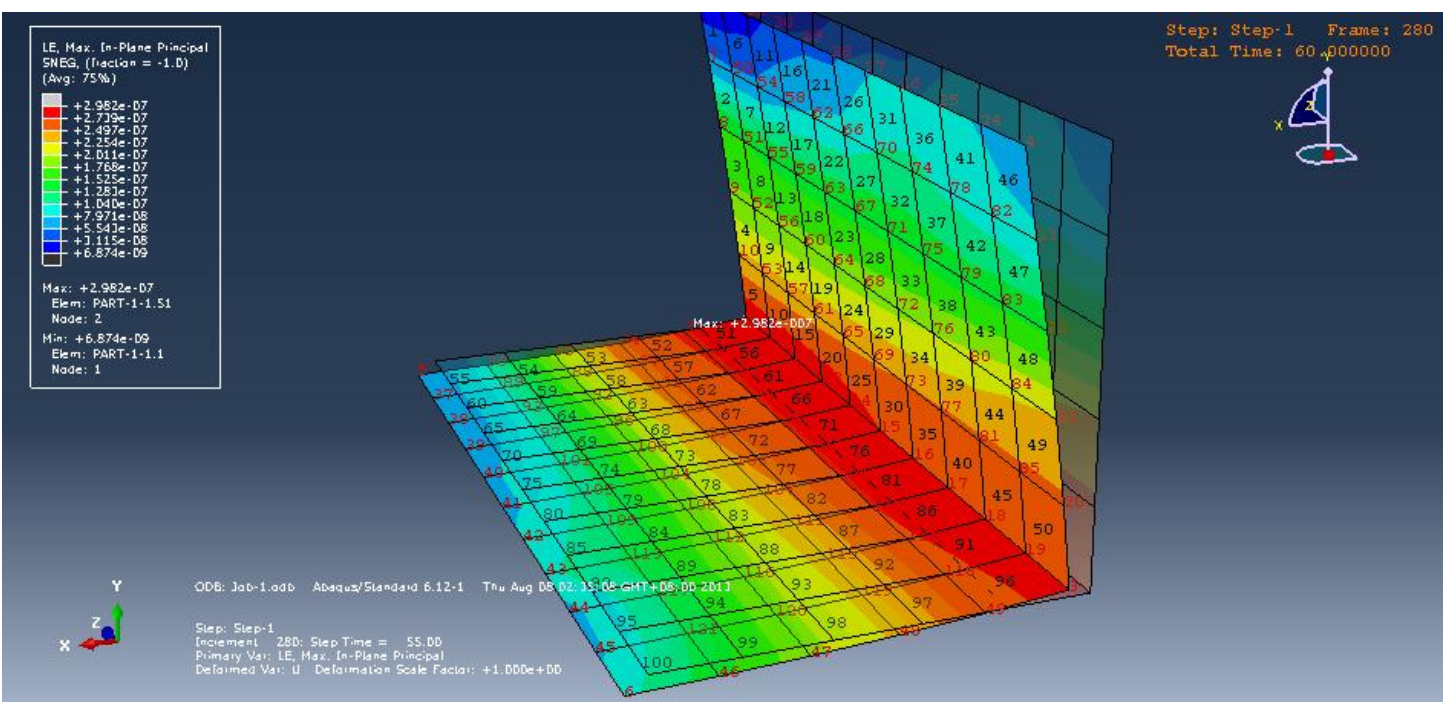

Fig. 82 strain distribution for case 3

\subsubsection{Case 4, Curve membrane under constant solar pressure}

In this case the original geometry is semi-cylinder, as we can see in Fig. 83. The solar pressure, lasting for 60 seconds, is applied to the membrane from negative $\mathrm{Y}$ direction, defined with respect to the original body. Considering the essence of solar pressure is particles collision, it is no surprise having the load distribution shown in Fig. 83.

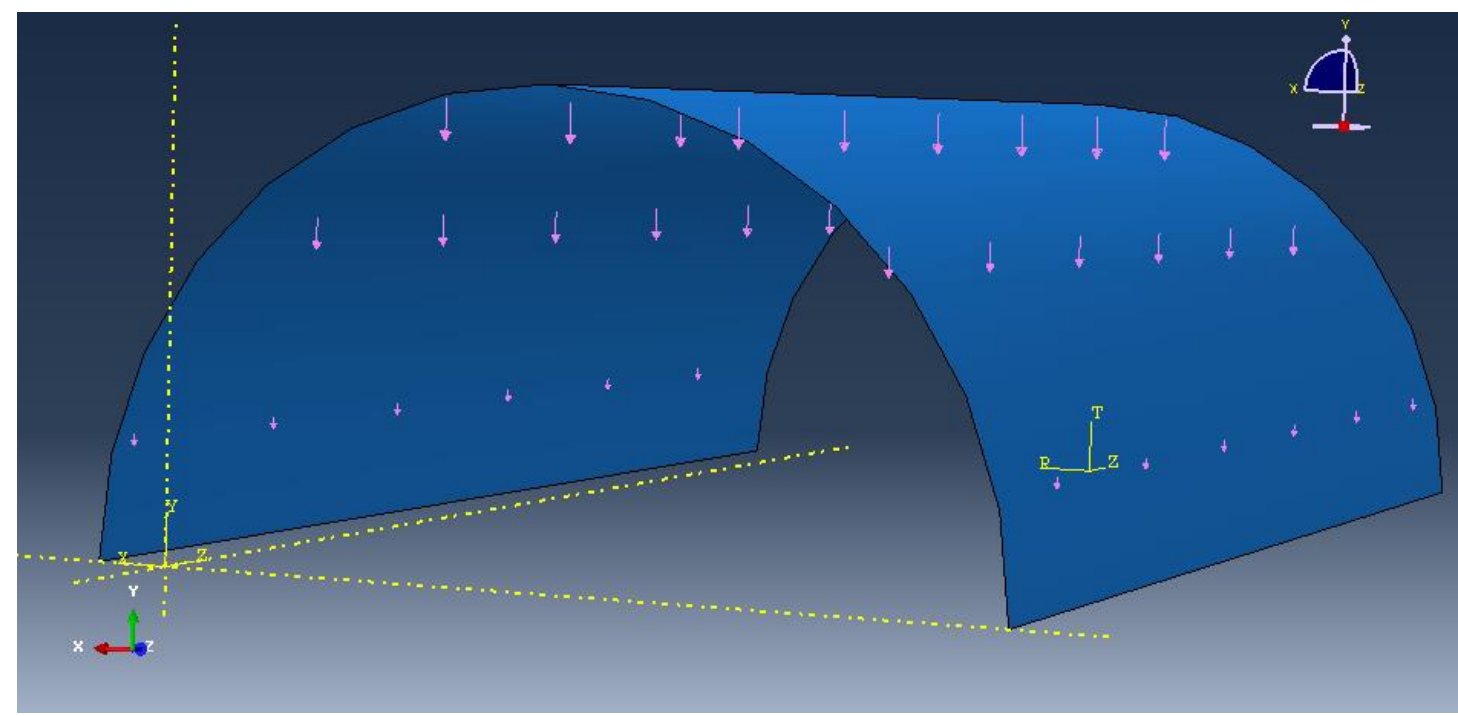

Fig. 83 force conditon for case 4

The following three Figures show the simulation results for this case. Fig. 84 shows the displacement distribution after 60 seconds. As it marked out, the maximum and minimum displacement is $\max =2.907 \times 10^{-3}, \min =2.496 \times 10^{-3}$, respectively. Fig. 85 and Fig. 86 shows the distribution of stress and strain at 60 second, respectively, also marking out the max and min value. 


Dynamics of flexible MLI-type debris for accurate orbit prediction

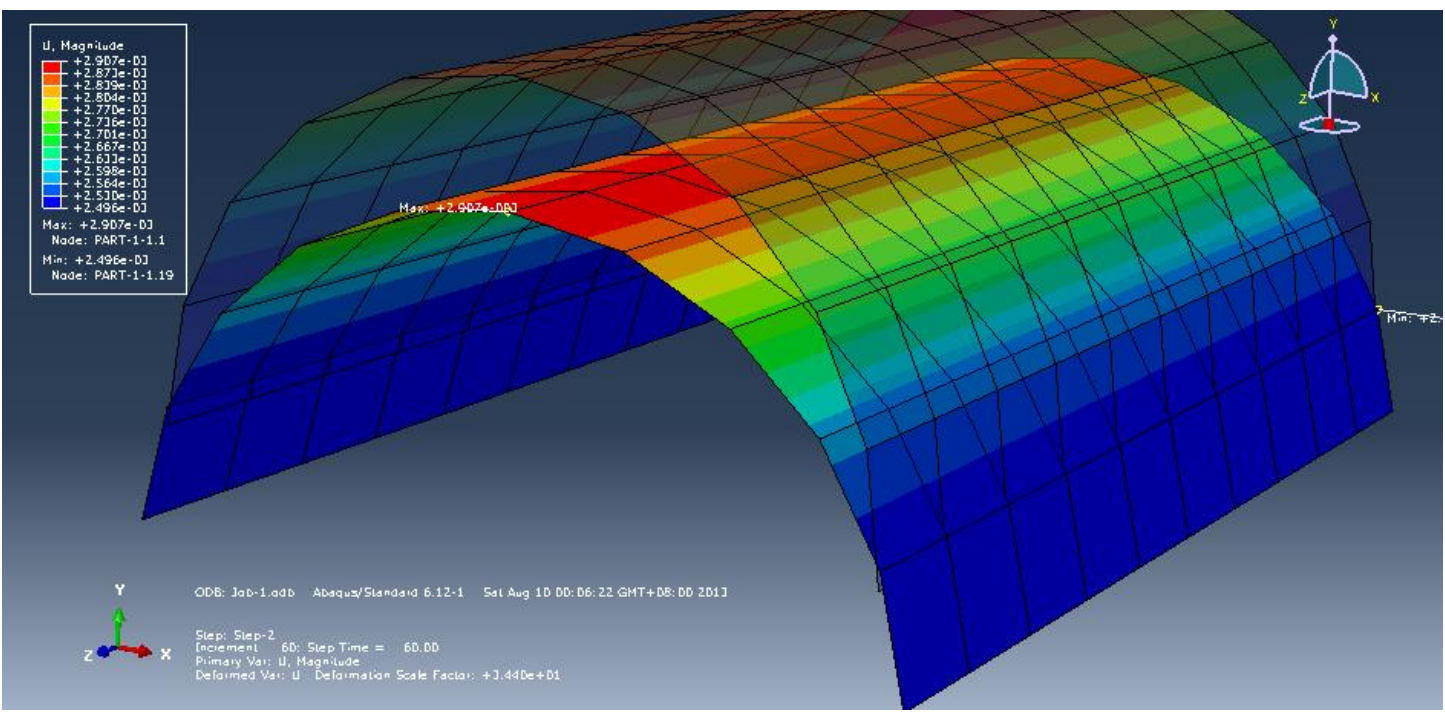

Fig. 84 displacement distribution for case 4

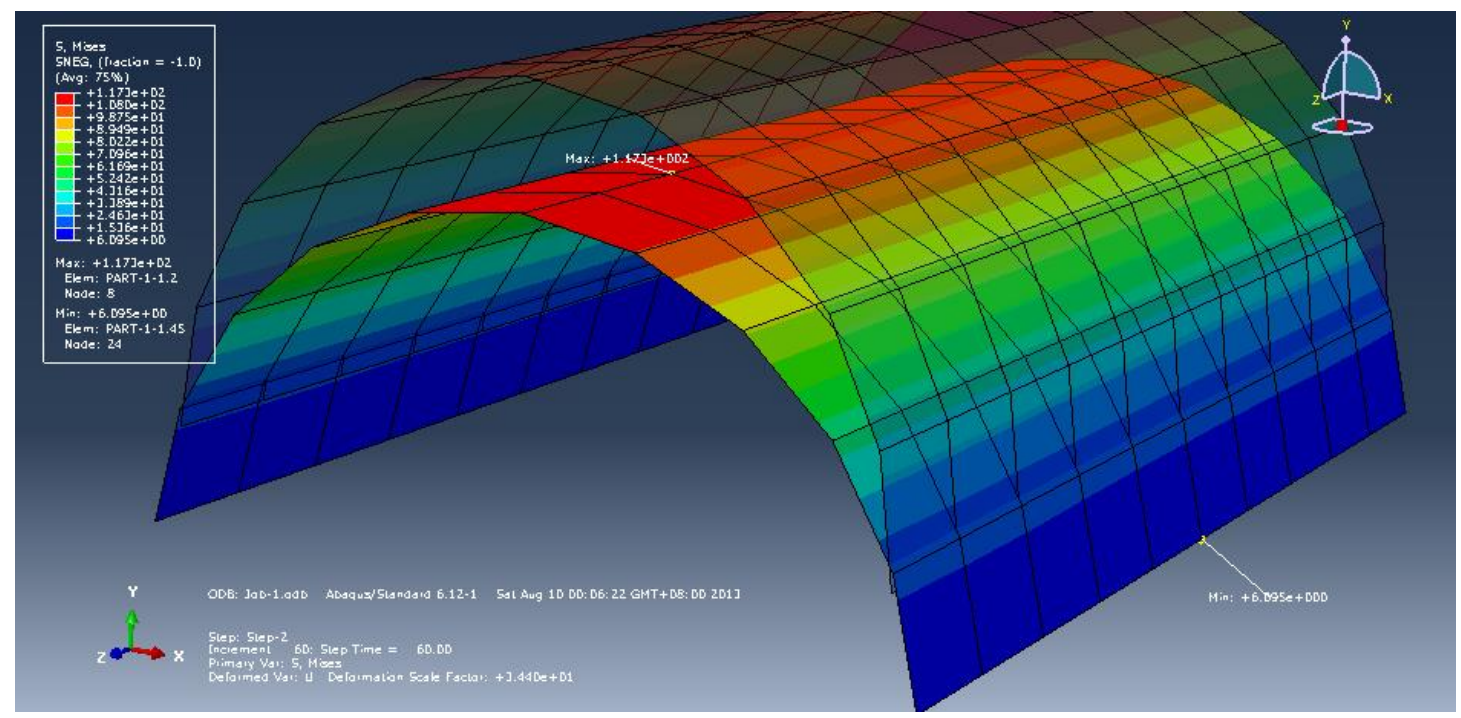

Fig. 85. stress distribution for case 4 


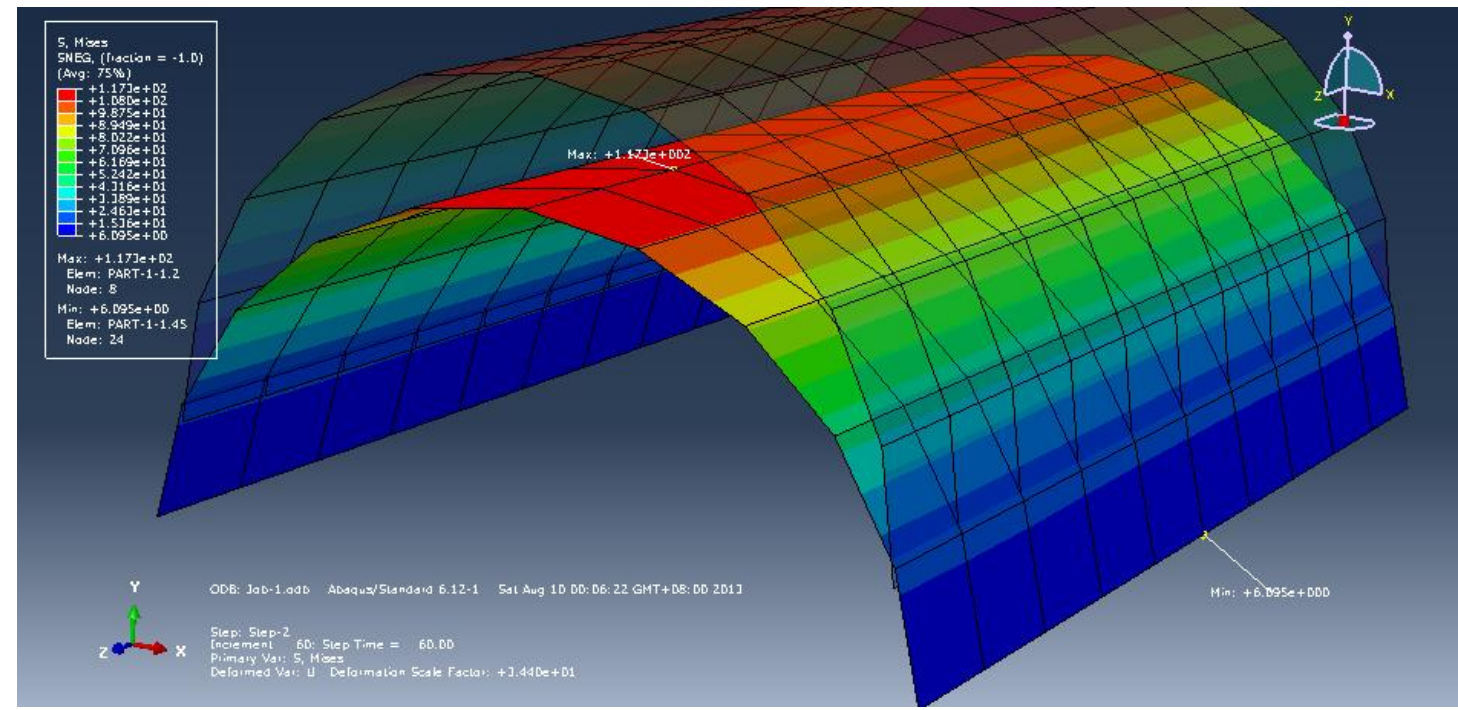

Fig. 86 strain distribution for case 4

\subsubsection{Case 5, Curve membrane under changeable load}

Like Case 3, we assume in the first five seconds, beside the solar pressure, there is another face load, having the same characteristic of solar pressure, applied on the membrane from negative $\mathrm{X}$ direction, as shown in Fig. 87, and its magnitude is $10^{-6}$ pa.

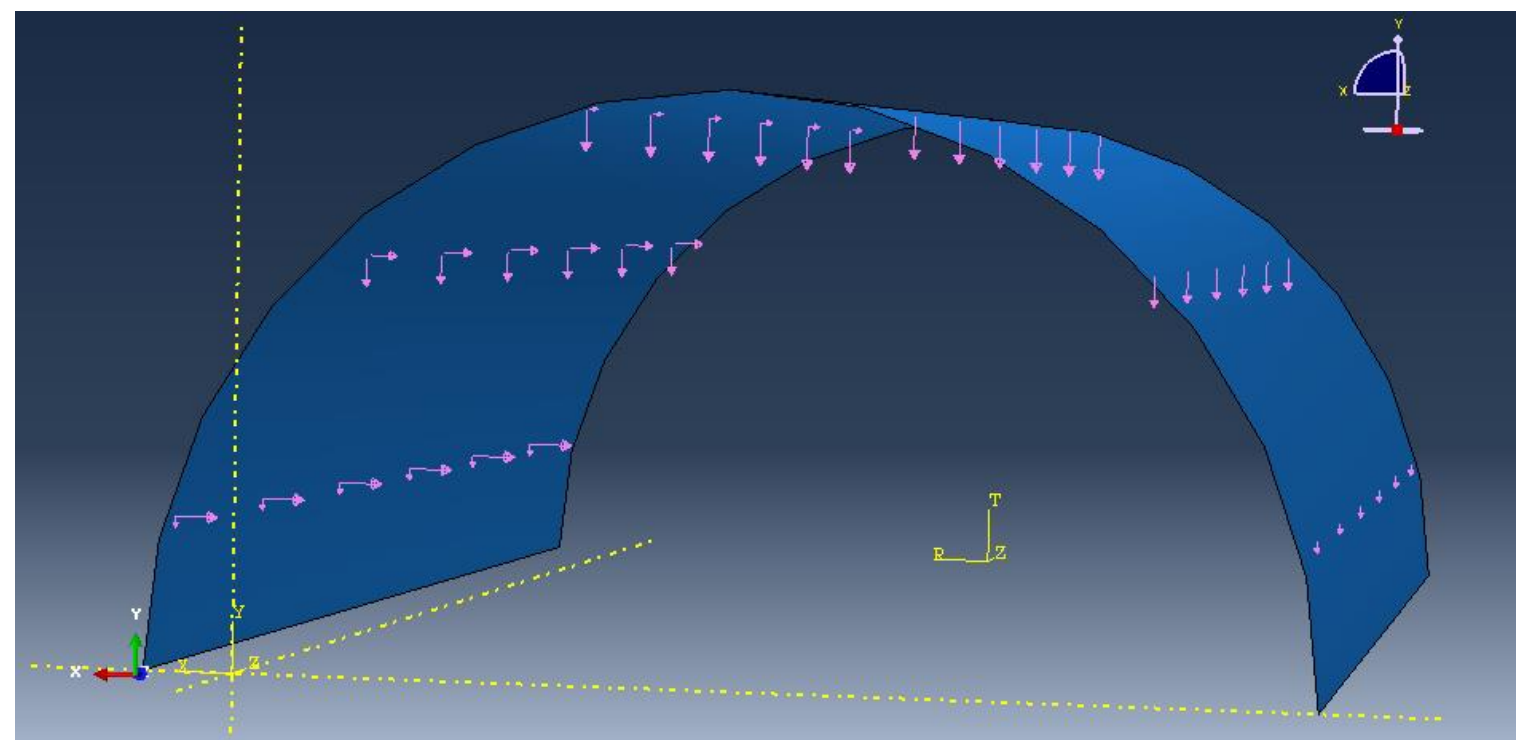

Fig. 87 force conditon for case 5

Similarly, Fig. 88 shows the displacement distribution in 60 seconds for this case, $\max =0.0502$ and $\min =0.0102$. Fig. 89. and Fig. 90 is the contours picture for stress as well as strain. Like Case 3, with a relatively large initial disturbing force, the membrane will perform a more obvious deformation, which in result will lead to larger and more complex displacement. 


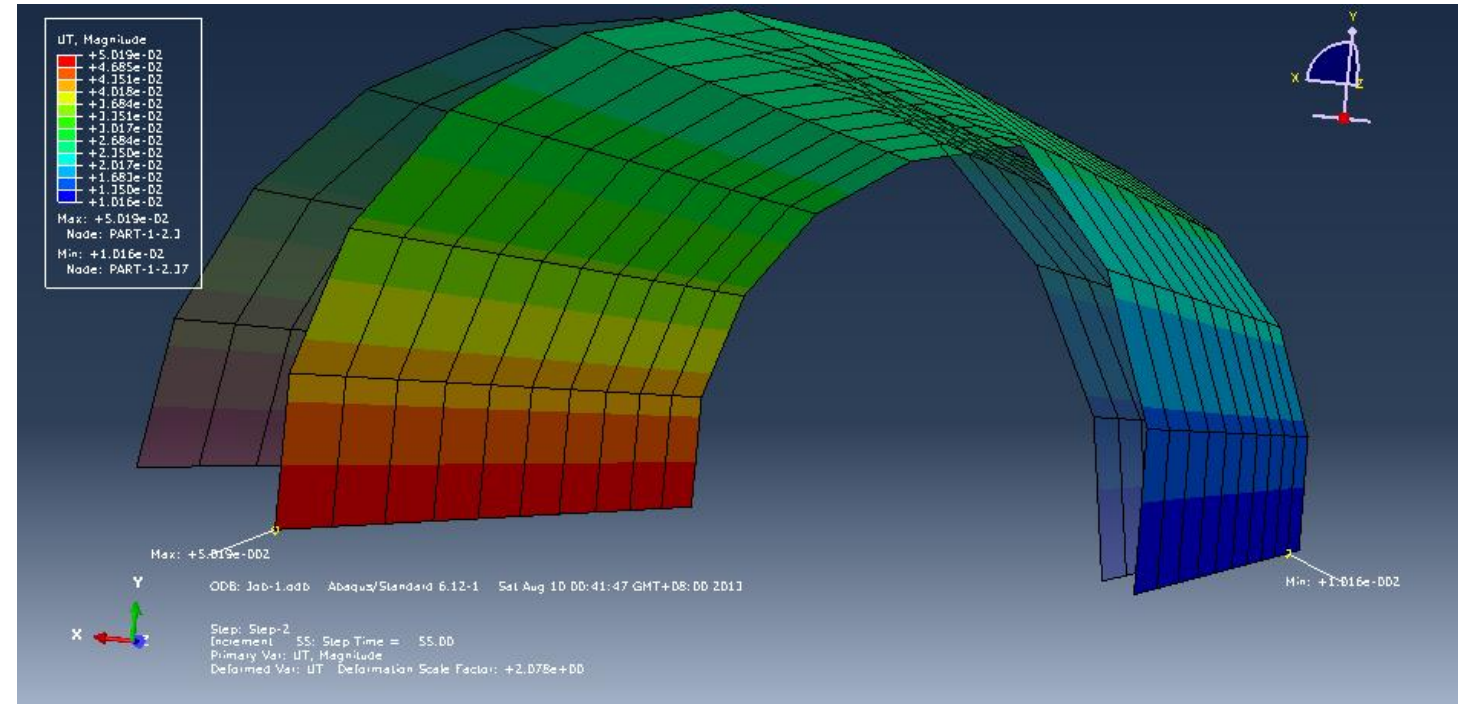

Fig. 88. displacement distribution for case 5

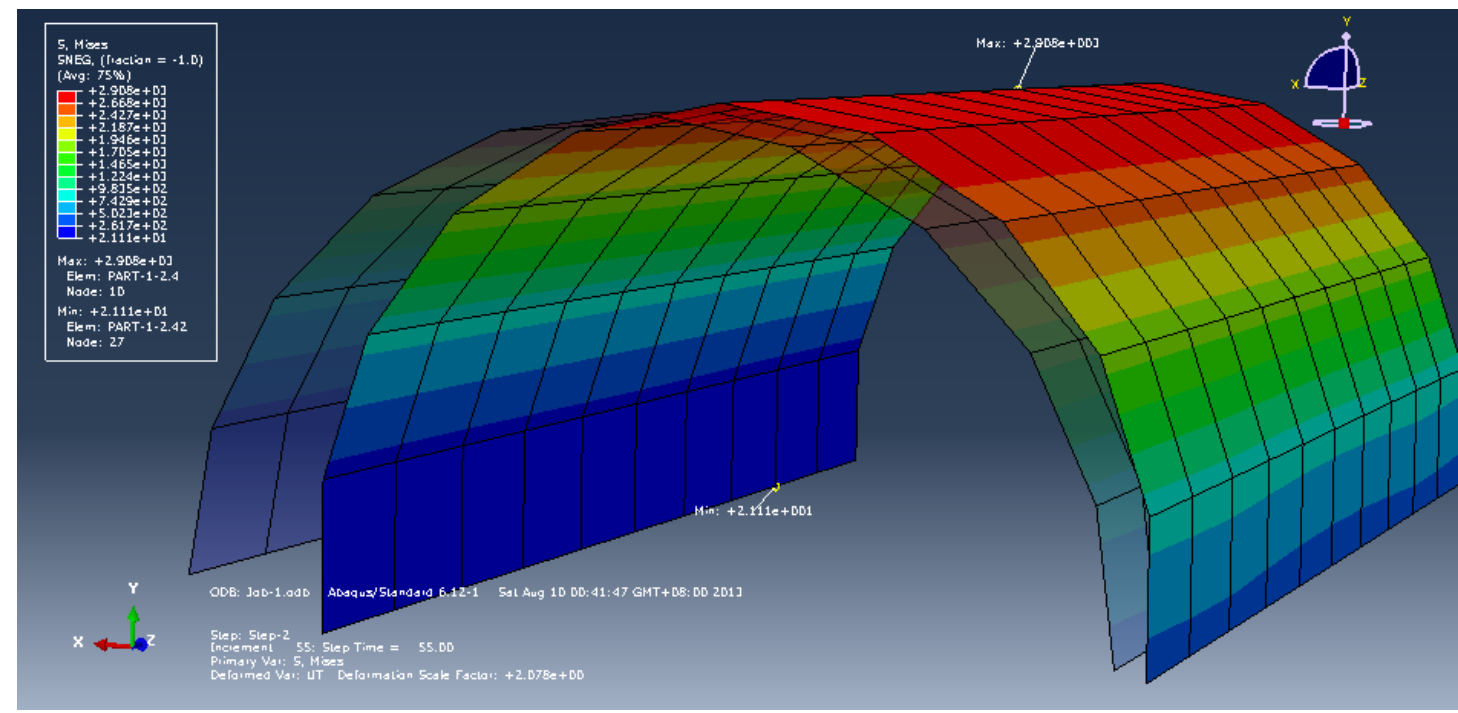

Fig. 89. Stress distribution for case 5 

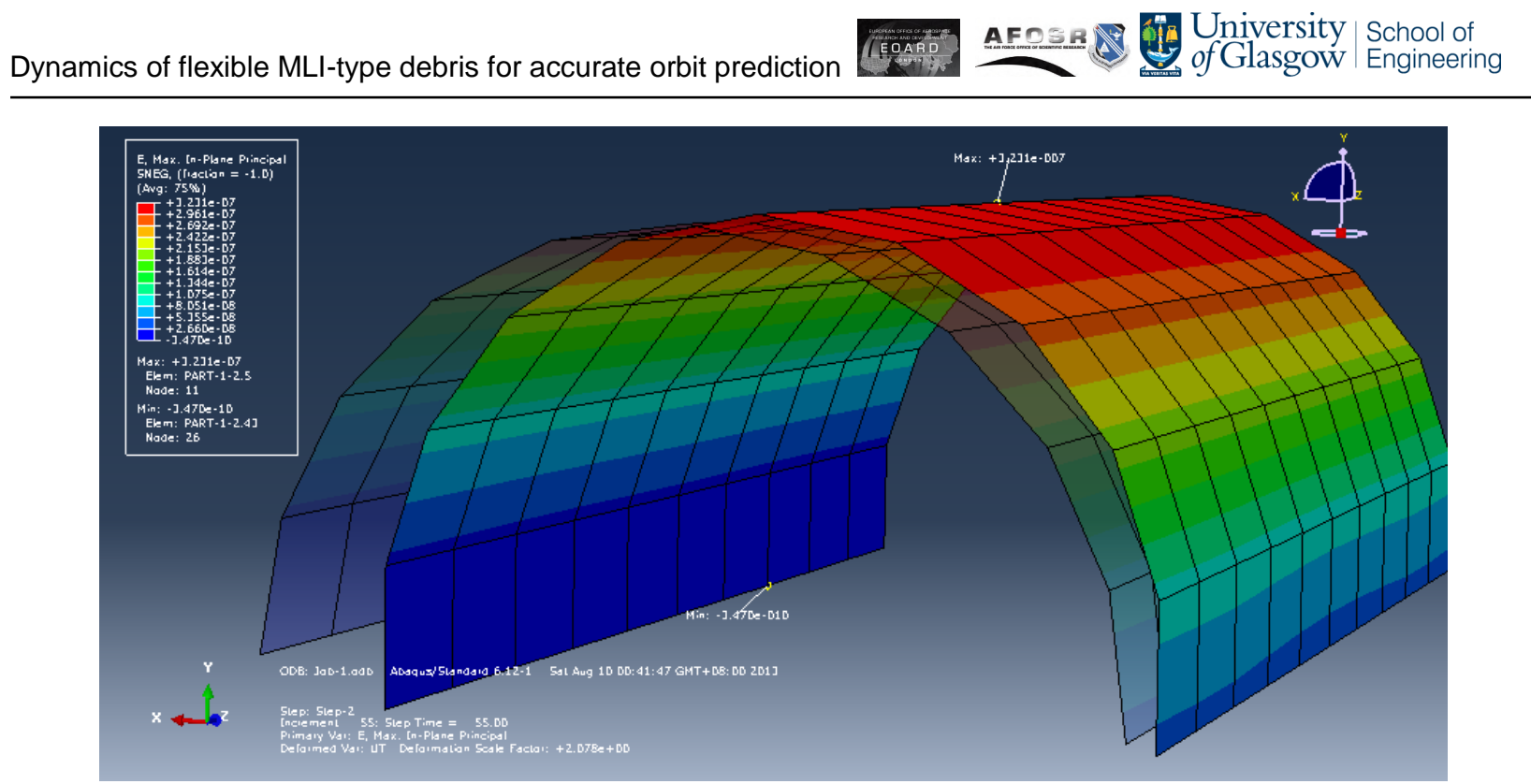

Fig. 90 strain distribution for case 5

\subsection{Conclusion}

In this report we use the properties of MLI to build a model for a simple square, thin-membrane structure. Then three origin geometries and five force conditions was set as a simulation of real cases. For every case, we use ABAQUS to simulate the motion the deformation and display the result in contours pictures.

From those results we can find in space even very small force lasts for very short period will have a nonnegligible influence on the thin flexible membranes, especially considering their deformation. The very easily happened deformation will change their load condition and in turn change the motion law of those on orbit membranes. Another interesting finding is the contours pictures for stress and strain is always the same which means the deformed structure will always have the same distribution on stress and strain.

Actually, what we have done now is just a starter of the study. In order to build up an accurate model to describe the deformation as well as attitude and orbit changing of on orbit membrane, there is a lot more need to improve and prefect. As we know it is a long haul, usually measured by hours or days, that complex loads in space influence the on orbit membrane. In our analysis, however, the duration of the load is quite short and the load condition we considered is also simplified, mainly solar pressure and a simple disturbing force in two cases. Therefore, in next step, it is necessary to lengthen the load duration and build a more accurate load condition model based on the location of the membrane on the orbit. Besides, more complicated structure and origin geometries should be considered. 


\section{References}

1. Desmond, B. Accessing China's ASAT program. 2007; Available from: http://rmit.nautilus.org/forum-reports/0714sball/fig3-debris.html.

2. $\quad$ Tariq, M. Debris From Space Collision Poses Threat to Other Satellites. 2009.

3. Anz-Meador, P.D. and M.J. Matney, An assessment of the NASA explosion fragmentation model to $1 \mathrm{~mm}$ characteristic sizes. Advances in Space Research, 2004. 34: p. 987-992.

4. Yi-yong, L., S. Huai-rong, and L. Zhi, Faster algorithm of debris cloud orbital character from spacecraft collision breakup. Advances in Space Research, 2009. 43: p. 1527-1531.

5. Krisko, P.H. and D.T. Hall, Geosynchronous region orbital debris modeling with GEO_EVOLVE 2.0. Advances in Space Research, 2004. 34: p. 1166-1170.

6. Liou, J.-C., et al., LEGEND - a three-dimensional LEO-to-GEO debris evolutionary model. Advances in Space Research, 2004. 34: p. 981-986.

7. Klinkrad, H., et al. An Introduction to the 1997 ESA Master Model. in Second European Conference on Space Debris. 1997. Darmstadt, Germany.

8. Gelhaus, J., et al. Validation of the ESA-MASTER-2009 Space Debris population. in 61st International Astronautical Congress (IAC 2010). 2010.

9. Jehn, R., et al., Estimating The Number of Debris in The Geostationary Ring. Acta Astronautica, 2006. 59: p. 84-90.

10. Celestino, C.C., O.C. Winter, and A.F.B.A. Prado, Debris perturbed by radiation pressure: relative velocities across circular orbits. Advances in Space Research, 2004. 34(5): p. 1177-1180.

11. Johnson, N.L., Space Debris Mitigation Strategies and Practices in Geosynchronous Transfer Orbits. Advances in Space Research, 2005. 35: p. 1328-1334.

12. McKnight, D.S. and F.R.D. Pentino, New insight on the orbital debris collision hazard at GEO. Acta Astronautica, 2013. 85: p. 73-82.

13. Schildknecht, T., R. Musci, and T. Flohrer, Properties of the high area-to-mass ratio space debris population at high altitudes. Advances in Space Research, 2008. 41(5): p. 1039-1045.

14. Schildknecht, T., et al. An Optical Search for Small-Size Debris in GEO and GTO. in AMOS Technical Conference. 2003. Maui, Hawaii, USA.

15. Schildknecht, et al. European Efforts to Survey, Track, and Characterize Small-Size Objects at High Altitudes.

16. Dever, J.A., et al., Mechanical properties degradation of TEFLON FEP returned from the hubble space telescope, in 36 th Aerospace Sciences Meeting \& Exhibit. 1998.

17. Murakami, J., et al., Micro-satellite impact tests to investigate multi-layer insulation fragments, in Space Systems Dynamics Laboratory. 2008, Kyushu University.

18. Anselmo, L. and C. Pardini, Long-term dynamical evolution of high area-to-mass ratio debris released into high earth orbits. Acta Astronautica, 2010. 67: p. 204-216.

19. Hanada, T. and J.-C. Liou, Theoretical and Empirical Analysis of the Average Cross-sectional Areas of Breakup Fragments. Advances Space in Research, 2011. 47(9): p. 1480-1489.

20. Mozurkewich, D., Toward Realistic Dynamics of Rotating Orbital Debris and Implications for Lightcurve Interpretation, in Advanced Maui Optical and Space Surveillance Technologies Conference. 2011: Maui, Hawaii.

21. $\quad$ Flegel, S.K., et al., Multi-layer insulation model for MASTER-2009. Acta Astronautica, 2011. 69.

22. Kelecy, T. and M. Jah, Analysis of high area-to-mass ratio (HAMR) GEO space object orbit determination and prediction performance: Initial strategies to recover and predict HAMR GEO trajectories with no a priori information. Acta Astronautica, 2011. 69: p. 551-558.

23. Kelecy, T., et al. Solar radiation pressure estimation and analysis of a GEO class of high area-to-mass ratio debris objects. in Astrodynamics 2007 - Advances in the Astronautical Sciences, Proceedings of the AAS/AIAA Astrodynamics Specialist Conference. 2008. P.O. Box 28130, San Diego, CA 92198, United States: Univelt Inc.

24. Valk, S. and A. Lemaître, Semi-analytical investigations of high area-to-mass ratio geosynchronous space debris including Earth's shadowing effects. Advances in Space Research, 2008. 42.

25. Hubaux, C., et al., Influence of Earth's shadowing effects on space debris stability. Advances in Space Research, 2012.

26. S., V., et al., Global dynamics of high area-to-mass ratios GEO space debris by means of the MEGNO indicator. Advances in Space Research, 2009. 43: p. 1509-1526.

27. Rosengren, A.J. and D.J. Scheeres. Long-term dynamics of high area-to-mass ratio space debris in GEO. in 63rd International Astronautical Congress (IAC 2012). 2012. Naples, Italy.

28. Früh, C. and T. Schildknecht, Variation of Area-to-Mass-Ratio of HAMR Space Debris objects. Monthly Notices of the Royal Astronomical Society, 2012. 419(4): p. 3521-3528.

29. Früh, C., T.M. Kelecy, and M.K. Jah. Attitude Dynamics Simulation of MLI Space Debris Ojects in Geosynchronous Earth Orbits. in AIAA/AAS Astrodynamics Specialist Conference 2012. Minneapolis. 
30. Vallado, D.A., Fundamentals of Astrodynamics and Applications. 3rd Edition ed. The Space Technology Library, ed. J.R. Wertz. 2007, El Segundo, California: Microcosm Press/Kluwer Academic Publishers.

31. Wertz, J.R., Spacecraft Attitude Determination and Control. Vol. 73. 1978, Dordrecht, Holland: D.Reidel Publishing Company.

32. Schaub, H. and J.L. Junkins, Analytical Mechanics of Space Systems, 2nd Edition AIAA Education Series, ed. J.A. Schetz. 2009, Reston, VA, USA

33. Channumsin, S., et al. Orbital dynamics of lightweight flexible debris. in 64th International Astronautical Congress (IAC 2013). 2013. Beijing, China: IAF.

34. Ross, C.T.F., Advanced applied finite element methods. 1998, West Sussex, UK: Horwood Publishing Chichester.

35. Rayleigh, L., Theory of Sound. Vol. 2. 1877, New York: Dover.

36. Sheldahl. The red book. Available from: http://www.sheldahl.com/documents/RedBook.pdf.

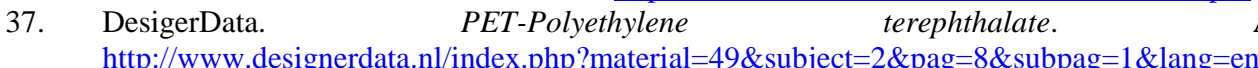

Available from:

38. Mott, P.H. and C.M. Roland, Limits to Poisson's ratio in isotropic materials. Physical Review B, 2009. 80(13): p. 132104.

39. Goodfellow 2014.

40. Blinn, J.F., Jim blinn's corner: Me and my (fake) shadow. IEEE Computer Graphics \& Applications8., 1988: p. 82-86.

41. Eisemann, E., et al., eds. Real-time shadows. 2012, CRC press

Taylor \& Francis Group.

42. Sheldahl, The red book, http://www.sheldahl.com/documents/RedBook\%20revised\%2020-AUG\%20\%202012.pdf, Editor. 2012.

43. Ojakangas, G.W. and N. Hill, Toward Realistic Dynamics of Rotating Orbital Debris and Implications for Lightcurve Interpretation. Advanced Maui Optical and Space Surveillance Technologies Conference, Maui, Hawaii, 2011.

44. Woodburn, J. Effects of eclipse boundary crossing on the numerical integration of orbit trajectories.

45. Woodburn, J. Mitigation of the effects of eclipse boundary crossing on the numerical integration of orbit trajectories using an Encke Type Correction Algorithm. 2001. AIAA/AAS.

46. Woodburn, J. and S. Tanygin. Efficient numerical integration of coupled orbit and attitude trajectories using an Encke type correction algorithm. in AAS/AIAA Astrodynamics Conference. 2002. Quebec City, Que., Canada: Univelt Inc.

47. Ascher, U.M. and L.R. Petzold, Computer Methods for Ordinary Differential Equations and Differential-Algebraic Equations. 1998: Society for Industrial and Applied Mathematics.

48. Curtis, H., Orbital mechanics for engineering students (3rd ed.). 2013, Oxford: Butterworth-Heinemann.

49. Berry, M.M., A Variable-Step Double-Integration Multi-Step Integrator. 2004, Virginia Polytechnic Institute and State University: Blacksburg, Virginia.

50. Kværnø, A. and P. Rentrop, Low Order Multirate Runge-Kutta Methods in Electric Circuit Simulation. 1999.

51. Fagan, M.J., Finite Element Analysis. 1992: Longman Group UK limited.

52. ABAQUS user manual. 2012. 